\title{
Geometria dos Exemplos de Katok
}

\author{
Ana Kelly de Oliveira \\ DISSERTAÇÃO APRESENTADA \\ AO \\ Instituto de MATEMÁtiCA E EstatístiCA \\ DA \\ UNIVERSIDADE DE SÃo PAULO \\ PARA \\ OBTENÇÃO DO TÍTULO \\ $\mathrm{DE}$ \\ Mestre em MatemáticA
}

Programa: Matemática

Orientador: Prof. Dr. Pedro Antonio Santoro Salomão

São Paulo, Dezembro de 2016 


\section{Geometria dos Exemplos de Katok}

Esta versão da dissertação contém as correções e alterações sugeridas pela Comissão Julgadora durante a defesa da versão original do trabalho, realizada em 02/12/2016. Uma cópia da versão original está disponível no Instituto de Matemática e Estatística da Universidade de São Paulo.

Comissão Julgadora:

- Prof. Dr. Pedro Antonio Santoro Salomão (orientador) - IME-USP

- Prof. Dr. Marcos Martins Alexandrino da Silva - IME-USP

- Prof. Dr. Joachim Weber - UNICAMP 


\section{Agradecimentos}

Agradeço primeiramente a Deus por sua graça, misericórdia e por cuidar de mim. Segundo, a minha família por todo o apoio e força que tem me dado nessa etapa dos meus estudos. Não posso deixar de fazer menção a todos que me incentivaram e tornaram a minha vinda a São Paulo mais fácil, Sandra Maria, Janyeid, Arlane e Samir, a todos vocês, muito obrigada.

Também agradeço aos meus colegas que direta ou indiretamente me ajudaram a entender assuntos relacionados a esse trabalho. E por último, mas não menos importante, agradeço ao meu orientador, Prof. Pedro Salomão, pela disponibilidade que teve em esclarecer minhas dúvidas. 


\section{Resumo}

OLIVEIRA, A. K. Geometria dos Exemplos de Katok. 2016. 110 f. Dissertação (Mestrado) - Instituto de Matemática e Estatística, Universidade de São Paulo, São Paulo, 2016.

Estudamos exemplos de métricas Finsler simétricas e não-simétricas em $S^{n}, \mathbb{C} P^{n}$ e $\mathbb{H} P^{n}$ com uma quantidade finita de geodésicas fechadas ou com uma quantidade pequena de geodésicas fechadas "curtas". São os chamados exemplos de Katok. Usamos como referência o artigo Geometry of the Katok examples [Zi183] de Wolfgang Ziller. Verificamos que existem métricas Finsler cujo número de geodésicas fechadas é $2 n$ (no caso de $S^{2 n}$ e $\left.S^{2 n-1}\right), n(n+1)$ (no caso de $\left.\mathbb{C} P^{n}\right)$ e $2 n(n+1)$ (no caso de $\mathbb{H} P^{n}$ ). Tais exemplos são construídos numa vizinhança qualquer da métrica Riemanniana canônica dessas variedades.

Palavras-chave: métrica Finsler, geodésicas fechadas, exemplo de Katok. 


\section{Abstract}

OLIVEIRA, A. K. Geometry of the Katok Examples. 2016. 110 f. Dissertação (Mestrado) - Instituto de Matemática e Estatística, Universidade de São Paulo, São Paulo, 2016.

We study examples of symmetric and non-symmetric Finsler metrics on $S^{n}, \mathbb{C} P^{n}$ and $\mathbb{H} P^{n}$ with a finite number of closed geodesics or with a small number of "short"closed geodesics. These are the well known Katok's examples. We use Ziller's article Geometry of the Katok examples [Zi183]. We exhibit Finsler metrics whose number of closed geodesics is $2 n$ (in the case of $S^{2 n}$ and $S^{2 n-1}$ ), $n(n+1)$ (in the case of $\mathbb{C} P^{n}$ ) and $2 n(n+1)$ (in the case of $\mathbb{H} P^{n}$ ). Such examples are found in any neighborhood of the canonical Riemannian metric on these manifolds.

Keywords: Finsler metric, closed geodesic, Katok’s example. 


\section{Sumário}

$\begin{array}{ll}\text { Lista de Figuras } & \text { ix }\end{array}$

$\begin{array}{ll}\text { Introdução } & 1\end{array}$

1 Métrica Finsler $\quad 3$

1.1 Espaços de Minkowski . . . . . . . . . . . . . . . . . . 3

1.2 Métrica Finsler . . . . . . . . . . . . . . . . . . . . . 9

1.2.1 Métricas Riemannianas . . . . . . . . . . . . . . . . . . . . . . . . 12

1.2 .2 Métrica dual . . . . . . . . . . . . . . . . . . 13

1.2.3 Métricas do tipo Randers . . . . . . . . . . . . . . . . . . . . . . . . . . 14

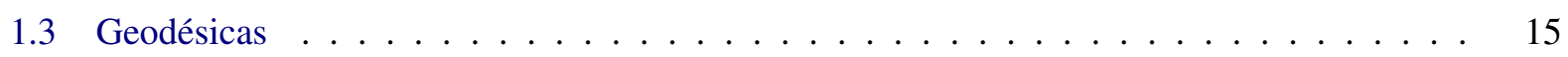

1.4 Derivada Covariante . . . . . . . . . . . . . . . . . . . . . . 17

1.5 Campos de Jacobi . . . . . . . . . . . . . . . . . . . . . 18

2 Espaços Projetivos e Grupos de Isometrias $\quad 21$

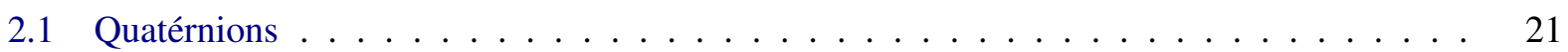

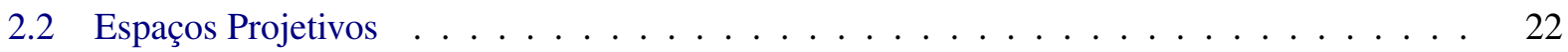

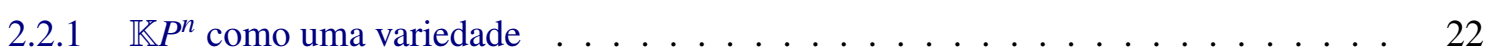

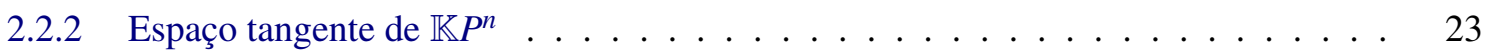

2.2 .3 Métrica Riemanniana Canônica em $\mathbb{K} P^{n} \ldots \ldots \ldots \ldots \ldots \ldots$

2.2 .4 Grupo de Isometrias em $\mathbb{K} P^{n} \ldots \ldots \ldots \ldots \ldots \ldots$

2.2 .5 Geodésicas em $\mathbb{K} P^{n} \ldots \ldots \ldots \ldots \ldots \ldots \ldots$

3 Cohomologia de De Rham 2

3.1 Cadeias e Cocadeias . . . . . . . . . . . . . . . . . . . . . . 25

3.2 Complexos de De Rham . . . . . . . . . . . . . . . . . . . . . . 26

3.3 Lema de Poincaré . . . . . . . . . . . . . . . . . . . . . . . . . . . . 28

3.4 Cohomologia de De Rham com suporte compacto e Cohomologia compacta vertical _. . 30

3.5 Classe de Euler . . . . . . . . . . . . . . . . . . . . . . . . . . 31

3.6 Sequência de Gysin . . . . . . . . . . . . . . . . . . . . . . . . . . . 33

3.7 Aplicações . . . . . . . . . . . . . . . . . . . . . . . . . 34

4 Variedades Simpléticas e Sistemas Hamiltonianos 4

4.1 Álgebra Linear Simplética . . . . . . . . . . . . . . . . . . . . . . . . . . . . . . . . . . . . . . . . . . . 45

4.2 Variedades Simpléticas . . . . . . . . . . . . . . . . . . . . . . 47

4.3 Equações de Hamilton $\ldots \ldots \ldots \ldots$. . . . . . . . . . . . . . . . . 48 


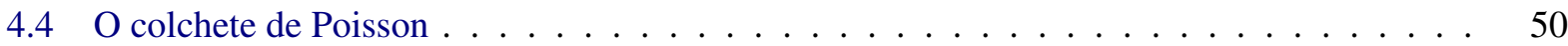

4.5 Fluxo Hamiltoniano no Fibrado Cotangente . . . . . . . . . . . . . . . . . . 51

4.6 Bifurcações e o Princípio de Hamilton . . . . . . . . . . . . . . . . . . . . . 54

$4.6 .1 \quad$ Princípio de Hamilton . . . . . . . . . . . . . . . . . . . 54

4.6 .2 Bifurcação de variedades críticas . . . . . . . . . . . . . . . . . . . . 60

5 Exemplos de Katok com quantidade finita de geodésicas fechadas $\quad \mathbf{6 5}$

5.1 Construção de Métricas Finsler e caracterização de geodésicas fechadas . . . . . . . . . . 65

5.2 Contando geodésicas fechadas . . . . . . . . . . . . . . . . . . . . . . 69

6 Propriedades Geométricas dos Exemplos de Katok $\quad 77$

6.1 Comprimento das Geodésicas . . . . . . . . . . . . . . . . . . . . . . . . 77

6.2 Aplicação de Poincaré dos Exemplos de Katok . . . . . . . . . . . . . . . . . . . 80

6.3 Índice de Morse dos Exemplos de Katok . . . . . . . . . . . . . . . . . . . . . . . 87

7 Exemplos de Katok Com Poucas Geodésicas Fechadas Curtas $\quad 93$

7.1 Geodésica como ponto crítico de uma função $\ldots \ldots \ldots \ldots \ldots$

7.2 Métricas Finsler Simétricas ． . . . . . . . . . . . . . . . . . . . . . . . . . . . . . 99

7.3 Aplicação de Poincaré das geodésicas fechadas curtas . . . . . . . . . . . . . . 106

$\begin{array}{ll}\text { Referências Bibliográficas } & 109\end{array}$ 


\section{Lista de Figuras}

$5.1 \quad$ Exemplo de Katok em $S^{2} \ldots \ldots \ldots \ldots \ldots \ldots \ldots \ldots \ldots$

6.1 Aplicação de Poincaré . . . . . . . . . . . . . . . . . . . . . . . . . 80

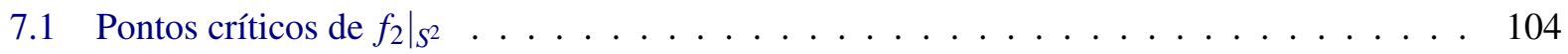




\section{Introdução}

Um problema clássico em cálculo de variações é estimar o número de pontos críticos de um funcional definido em um espaço de curvas fechadas numa variedade compacta. Em alguns casos, este problema pode ser traduzido para um problema de estimar o número de geodésicas fechadas de uma métrica Finsler apropriada na mesma variedade.

Em 1973 Katok encontrou algumas métricas Finsler não-simétricas em $S^{n}$ com uma quantidade finita de geodésicas. Em 1983 Ziller publicou o artigo [Zil83] estendendo tais exemplos para as variedades compactas $\mathbb{C} P^{n}, \mathbb{H} P^{n}$ e $\mathbb{C} a P^{2}$. Nesse artigo, Ziller constrói métricas Finsler com uma quantidade finita de geodésicas fechadas e calcula vários invariantes dessas geodésicas.

Nesse trabalho iremos expor com mais detalhes os resultados do artigo de Ziller [Zil83] focando no caso particular das variedades $S^{n}, \mathbb{C} P^{n}$ e $\mathbb{H} P^{n}$. Os primeiros capítulos 1,2,3 e 4 são introdutórios com definições e resultados preliminares usados nos capítulos subsequentes 5, 6 e 7 .

No capítulo 1 definimos métricas Finsler e apresentamos alguns exemplos. Mostramos que se $H$ é uma co-norma Finsler numa variedade $M$, então $F:=H \circ \mathscr{L}_{\frac{1}{2} H^{2}}^{-1}$ é uma métrica Finsler em $M$, onde $\mathscr{L}_{H}: T^{*} M \rightarrow$ $T M$ é a transformada de Legendre associada a $H$ (veja Teorema 1.25). A bibliografia usada nesse capítulo é [She01, Dah06, SH13, BCS00].

No capítulo 2 definimos o espaço projetivo complexo e o espaço projetivo quaterniônico. Falamos brevemente da estrutura de variedade, do espaço tangente, da métrica Riemanniana, do grupo de isometria e das geodésicas desses espaços projetivos.

No capítulo 3 calculamos as cohomologias dos espaços $T_{1} M$ e $C=T_{1} M / S^{1}$ para as variedades $M=$ $S^{n}, \mathbb{C} P^{n}, \mathbb{H} P^{n}$, necessárias para a demonstração do Teorema 5.25. A bibliografia usada nesse capítulo foi [BT24].

No capítulo 4 mostramos resultados sobre sistemas Hamiltonianos em variedades simpléticas que serão a base para as construções feitas nos capítulos 6,7 e 8 .

No capítulo 5 explicamos os exemplos de Katok e estimamos o menor número de geodésicas fechadas que existem para perturbações particulares da métrica Riemanniana canônica. Verificamos que os exemplos de Katok existem em qualquer vizinhança da métrica canônica em $S^{n}, \mathbb{C} P^{n}, \mathbb{H} P^{n}$ e que o menor número de geodésicas que obtemos nesses exemplos é $2 n$ (no caso de $S^{2 n}$ e $S^{2 n+1}$ ), $n(n+1)$ (no caso de $\mathbb{C} P^{n}$ ) e $2 n(n+1)$ (no caso de $\mathbb{H} P^{n}$ ). Mostramos também que qualquer métrica Finsler, suficientemente próxima da métrica Riemanniana canônica e com todas as geodésicas fechadas não-degeneradas, possui pelo menos este número de geodésicas fechadas.

No capítulo 6 calculamos o comprimento das geodésicas das métricas Finsler construídas no capítulo 5 e verificamos que todas as geodésicas fechadas são não-degeneradas e elíticas. Também calculamos a aplicação de Poincaré e o índice de Morse de todas estas finitas geodésicas fechadas.

Finalmente, no capítulo 8 generalizamos a construção de Katok para encontrar outros exemplos de métricas Finsler simétricas e não-simétricas com uma quantidade finita de geodésicas fechadas "curtas". 


\section{Capítulo 1}

\section{Métrica Finsler}

\subsection{Espaços de Minkowski}

Seja V um espaço linear real de dimensão finita.

Definição 1.1. Dizemos que a função contínua $F: V \rightarrow[0,+\infty)$ é uma norma de Minkowski em V se

(i) F é $C^{\infty}$ em $V \backslash\{0\}$,

(ii) $F(\lambda y)=\lambda F(y)$, para todo $y \in V$ e $\lambda>0$,

(iii) Para todo $y \in V \backslash\{0\}$ a forma bilinear simétrica

$$
g_{y}(u, v):=\left.\frac{1}{2} \frac{\partial^{2}}{\partial s \partial t} F^{2}(y+s u+t v)\right|_{s=t=0}
$$

é positiva definida.

Se F satisfaz a condição (ii), diz-se que F é positivamente homogênea de grau 1 e se $F^{2}$ satisfaz a condição (iii) diz-se que $F^{2}$ é fortemente convexa. O par $(V, F)$ é chamado de espaço de Minkowski.

Notação 1.2. Fixe uma base $\left\{e_{1}, \ldots, e_{n}\right\}$ para $V$. Se $u=u^{i} e_{i}$ e $v=v^{i} e_{i}$ então

$$
g_{y}(u, v)=g_{i j}(y) u^{i} v^{j}, \quad y=y^{i} e_{i}
$$

onde

$$
g_{i j}(y):=g_{y}\left(e_{i}, e_{j}\right)=\frac{1}{2} \frac{\partial^{2} F^{2}}{\partial y^{i} \partial y^{j}}(y) .
$$

Teorema 1.3 (Euler). Seja $h: \mathbb{R}^{n} \backslash\{0\} \rightarrow \mathbb{R}$ uma função suave e positivamente homogênea de grau $\alpha \in \mathbb{R}$, ou seja, $h(\lambda y)=\lambda^{\alpha} h(y)$ para todo $y \in \mathbb{R}^{n} \backslash\{0\}$ e $\lambda>0$. Então,

$$
h_{y^{i}}(y) y^{i}=\alpha h(y),
$$

para todo $y \neq 0$.

Demonstração. Como $y=\left(y^{1}, \ldots, y^{n}\right)$, derivando

$$
h\left(\lambda y^{1}, \ldots, \lambda y^{n}\right)=\lambda^{\alpha} h\left(y^{1}, \ldots, y^{n}\right)
$$

em relação a $\lambda$ temos

$$
\sum_{i=1}^{n} h_{y^{i}}(\lambda y) y^{i}=\alpha \lambda^{\alpha-1} h(y)
$$

Para $\lambda=1$, a equação (1.1) pode ser escrita na notação de Einstein como

$$
h_{y^{i}}(y) y^{i}=\alpha h(y)
$$


Observação 1.4. Se $h: \mathbb{R}^{n} \backslash\{0\} \rightarrow \mathbb{R}$ é uma função suave e positivamente homogênea de grau $\alpha \in \mathbb{R}$, isto é,

$$
h\left(\lambda y^{1}, \ldots, \lambda y^{n}\right)=\lambda^{\alpha} h\left(y^{1}, \ldots, y^{n}\right)
$$

então derivando em relação a $y^{i}$ temos

$$
\begin{aligned}
\lambda \frac{\partial h}{\partial y^{i}}\left(\lambda y^{1}, \ldots, \lambda y^{n}\right) & =\lambda^{\alpha} \frac{\partial h}{\partial y^{i}}\left(y^{1}, \ldots, y^{n}\right) \\
\frac{\partial h}{\partial y^{i}}\left(\lambda y^{1}, \ldots, \lambda y^{n}\right) & =\lambda^{\alpha-1} \frac{\partial h}{\partial y^{i}}\left(y^{1}, \ldots, y^{n}\right)
\end{aligned}
$$

isto é,

$$
\frac{\partial h}{\partial y^{i}}(\lambda y)=\lambda^{\alpha-1} \frac{\partial h}{\partial y^{i}}(y)
$$

Portanto, $\frac{\partial h}{\partial y^{i}}$ é positivamente homogênea de grau $\alpha-1$.

Observação 1.5. Seja $h$ uma função positivamente homogênea de grau 1. Então, pela Observação 1.4 temos

$$
\frac{\partial h}{\partial y^{i}}(\lambda y)=\frac{\partial h}{\partial y^{i}}(y)
$$

para todo $\lambda>0$. Logo, pelo Teorema de Euler temos

$$
\frac{\partial^{2} h}{\partial y^{j} \partial y^{i}}(y) y^{j}=0
$$

Observação 1.6. Dada uma norma de Minkowisk $F$ então $F^{2}$ é positivamente homogênea de grau $2 \mathrm{e} \frac{\partial F^{2}}{\partial y^{i}}$ é positivamente homogênea de grau 1. Usando o Teorema de Euler, obtemos

$$
\begin{aligned}
g_{i j}(y) y^{j} & =\frac{1}{2} \frac{\partial^{2} F^{2}}{\partial y^{i} \partial y^{j}}(y) y^{j} \\
& =\frac{1}{2} \frac{\partial^{2} F^{2}}{\partial y^{j} \partial y^{i}}(y) y^{j} \\
& =\frac{1}{2} \frac{\partial}{\partial y^{j}}\left(\frac{\partial F^{2}}{\partial y^{i}}\right)(y) y^{j} \\
& =\frac{1}{2} \frac{\partial F^{2}}{\partial y^{i}}(y) .
\end{aligned}
$$

Portanto,

$$
g_{y}(y, v)=\frac{1}{2} d F^{2}(y) \cdot v .
$$

Observação 1.7. Seja $h: \mathbb{R}^{n} \backslash\{0\} \rightarrow \mathbb{R}$ uma função suave e positivamente homogênea de grau 2. Usando o Teorema de Euler e a Observação 1.4 temos

$$
\begin{aligned}
\frac{\partial^{2} h}{\partial y^{i} \partial y^{j}}(y) y^{i} y^{j} & =\frac{\partial}{\partial y^{i}}\left(\frac{\partial h}{\partial y^{j}}\right)(y) y^{i} y^{j} \\
& =\frac{\partial h}{\partial y^{j}}(y) y^{j} \\
& =2 h(y)
\end{aligned}
$$


Portanto, para a norma de Minkowski F segue que

$$
g(y)(y, y)=g_{i j}(y) y^{i} y^{j}=\frac{1}{2} \frac{\partial^{2} F^{2}}{\partial y^{i} \partial y^{j}}(y) y^{i} y^{j}=F^{2}(y) .
$$

Lema 1.8. Seja $(V, F)$ um espaço de Minkowski. Então,

(i) $F(y)>0$ se $y \in V \backslash\{0\}$,

(ii) $F(0)=0$.

Demonstração. Seja $y \in V$ não nulo. Pela Observação 1.7 e por $g_{y}$ ser uma forma bilinear positiva definida segue que

$$
F^{2}(y)=g_{y}(y, y)>0 \Longrightarrow F(y) \neq 0 .
$$

Logo, como $F$ é uma função não negativa obtemos $F(y)>0$. Agora, tome uma sequência $\left(\lambda_{n}\right)$ de números reais positivos tal que $\lambda_{n} \rightarrow 0$. Como $F$ é contínua temos

$$
F(0)=\lim _{n \rightarrow 0} F\left(\lambda_{n} y\right)=\lim _{n \rightarrow 0} \lambda_{n} F(y)=0 .
$$

Lema 1.9 ([She01], pág 9). Seja (V,F) um espaço de Minkowski. Para $v, w \in V$ temos

$$
F(v+w) \leq F(v)+F(w) .
$$

A igualdade vale se, e somente se, $w=\lambda v$ para algum $\lambda \geq 0$.

Lema 1.10 ([She01], pág 10). Seja $(V, F)$ um espaço de Minkowski. Para qualquer $y \neq 0$,

$$
g_{y}(y, v) \leq F(y) F(v),
$$

para todo $v \in V$. A igualdade vale se, e somente se, $v=\lambda$ y para algum $\lambda \geq 0$.

Lema 1.11. Seja $(V, F)$ um espaço de Minkowski. Suponha que y,v $\in V \backslash\{0\}$ satisfaz.

$$
g_{y}(y, w)=g_{v}(v, w), \quad w \in V .
$$

Então, $y=v$.

Demonstração. Fazendo $w=y$ e $w=v$ temos

$$
\begin{aligned}
& F^{2}(y)=g_{v}(v, y) \leq F(v) F(y) \\
& F^{2}(v)=g_{y}(y, v) \leq F(y) F(v)
\end{aligned}
$$

então,

$$
F(y) \leq F(v) \leq F(y) \Rightarrow F(y)=F(v) .
$$

Assim,

$$
g_{v}(v, y)=F^{2}(y)=F(y) F(v)
$$

e pelo Lema 1.10 existe $\lambda \geq 0$ tal que $y=\lambda v$. Logo,

$$
g_{v}(v, y)=g_{v}(v, \lambda v)=\lambda g_{v}(v, v)=\lambda F^{2}(v)
$$

De (1.3), (1.4) e (1.5) segue que

$$
\lambda F^{2}(v)=g_{v}(v, y)=F(y) F(v)=F^{2}(v) \Rightarrow \lambda=1 .
$$

Portanto, $y=v$. 
Definição 1.12. A dual da norma de Minkowski é a função $F^{*}: V^{*} \rightarrow[0,+\infty)$ definida por

$$
F^{*}(\xi)=\max \{\xi(y) ; y \in V, F(y)=1\}, \xi \in V^{*} .
$$

Como $F^{-1}(1)=\{y \in V ; F(y)=1\}$ é compacto então a dual da norma de Minkowski está bem definida e é finita.

Definição 1.13. Seja $H: V \rightarrow[0,+\infty)$ uma função $C^{\infty}$. Defina $L_{H}: V \rightarrow V^{*}$ por $L_{H}(y)=d H(y)$.

Observação 1.14. Note que se F é uma norma de Minkowski, pela Observação 1.6 segue que

$$
L_{\frac{1}{2} F^{2}}(y)=\frac{1}{2} d F^{2}(y)=g_{y}(y, \cdot) .
$$

Proposição 1.15. A aplicação $L_{\frac{1}{2} F^{2}}: V \rightarrow V^{*}$ é uma bijeção.

Demonstração. Observe que

$$
0=L_{\frac{1}{2} F^{2}}(y)=g_{y}(y, \cdot) \Leftrightarrow y=0,
$$

pois $g_{y}$ é uma forma bilinear positiva-definida e isso implica que $g_{y}$ é uma forma bilinear não degenerada. Assim, é suficiente mostrar que $L_{\frac{1}{2} F^{2}}: V \backslash\{0\} \rightarrow V^{*} \backslash\{0\}$ é uma bijeção. O Lema 1.11 implica a injetividade. Para provar a sobrejetividade suponha que $\xi \in V^{*} \backslash\{0\}$. Seja $\lambda=F^{*}(\xi)$ e seja $y \in V$ tal que $F(y)=1$ e $\xi(y)=\lambda$. Defina $W_{y}:=\left\{w \in V ; g_{y}(y, w)=0\right\}$. Quero mostrar que se $w \in W_{y}$ então $\xi(w)=0$. Seja $\gamma$ : $(-\varepsilon, \varepsilon) \rightarrow F^{-1}(1)$ uma curva tal que

$$
\gamma(t)=\frac{y+t w}{F(y+t w)}, w \in W_{y}
$$

então, $\gamma(0)=y$. Como $y$ é um ponto crítico (de máximo) da aplicação $v \mapsto \xi(v)$ segue que

$$
\begin{aligned}
0 & =\left.\frac{d}{d t} \xi(\gamma(t))\right|_{t=0} \\
& =\xi\left(\frac{w F(y)}{F(y)^{2}}-\frac{y}{F(y)^{2}} \sum_{i} \frac{\partial F}{\partial y^{i}}(y) w^{i}\right) \\
& =\xi\left(w-y \sum_{i} \frac{\partial F}{\partial y^{i}}(y) w^{i}\right)
\end{aligned}
$$

Como $w \in W_{y}$ temos

$$
0=g_{y}(y, w)=\frac{1}{2} d F^{2}(y) w=F(y) \sum_{i} \frac{\partial F}{\partial y^{i}}(y) w^{i}=\sum_{i} \frac{\partial F}{\partial y^{i}}(y) w^{i} .
$$

Logo, $\xi(w)=0$. Observe que para qualquer $v \in V$ temos

$$
w=v-g_{y}(y, v) y \in W_{y}
$$

pois como $F(y)=1$ temos

$$
g_{y}(y, w)=g_{y}(y, v)-g_{y}(y, v) g_{y}(y, y)=g_{y}(y, v)-g_{y}(y, v) F^{2}(y)=0
$$

Então, para todo $v \in V$ temos

$$
v=w+g_{y}(y, v) y
$$


onde $w \in W_{y}$ é como em (1.6). Assim, para todo $v \in V$ temos

$$
\begin{aligned}
0 & =\xi(w) \\
& =\xi\left(v-g_{y}(y, v) y\right) \\
& =\xi(v)-g_{y}(y, v) \xi(y) \\
& =\xi(v)-g_{y}(y, v) \lambda \\
& =\xi(v)-g_{y}(\lambda y, v)
\end{aligned}
$$

ou seja,

$$
\xi(v)=g_{y}(\lambda y, v)=L_{\frac{1}{2} F^{2}}(\lambda y)(v) \quad \text { para todo } v \in V
$$

Logo, $\xi=L_{\frac{1}{2} F^{2}}(\lambda y)$. Portanto, $L_{\frac{1}{2} F^{2}}$ é uma bijeção.

Proposição 1.16. Sejam $F$ uma norma de Minkowski e $F^{*}$ sua norma dual. Então,

$$
F=F^{*} \circ L_{\frac{1}{2} F^{2}}
$$

Demonstração. Se $y=0$, então,

$$
F(y)=0 \text { e } F^{*} \circ L_{\frac{1}{2} F^{2}}=F^{*}(0)=0
$$

e a propriedade vale. Suponha que $y \neq 0$. Como $F^{2}=g_{y}(y, y)$ segue que

$$
F(y)=\frac{g_{y}(y, y)}{F(y)}=g_{y}\left(y, \frac{y}{F(y)}\right)=L_{\frac{1}{2} F^{2}}(y)\left(\frac{y}{F(y)}\right) \leq F^{*} \circ L_{\frac{1}{2} F^{2}} .
$$

Por outro lado, pelo Lema 1.10 temos

$$
F^{*} \circ L_{\frac{1}{2} F^{2}}(y)=\sup _{v \neq 0} L_{\frac{1}{2} F^{2}}\left(\frac{v}{F(v)}\right)=\sup _{v \neq 0} \frac{g_{y}(y, v)}{F(v)} \leq \sup _{v \neq 0} \frac{F(v) F(y)}{F(v)}=F(y) .
$$

Portanto,

$$
F=F^{*} \circ L_{\frac{1}{2} F^{2}}
$$

Proposição 1.17. Sejam $g^{i j}(y)$ a inversa de $g_{i j}(y) e$

$$
g^{* i j}(\xi)=\frac{1}{2} \frac{\partial^{2} F^{* 2}}{\partial \xi^{i} \partial \xi^{j}}(\xi), \quad \xi \in V^{*} \backslash\{0\} .
$$

Então,

$$
g^{i j}=g^{* i j} \circ L_{\frac{1}{2} F^{2}} .
$$

Demonstração. Diferenciando $\frac{1}{2} F^{2}=\frac{1}{2} F^{*} \circ L_{\frac{1}{2} F^{2}}$ com respeito a $y^{i}$ para $y \in V \backslash\{0\}$ temos

$$
\begin{aligned}
\frac{1}{2} \frac{\partial F^{2}}{\partial y^{i}}(y) & =\frac{1}{2} \frac{\partial}{\partial y^{i}}\left[F^{*} \circ L_{\frac{1}{2} F^{2}}\right](y) \\
& =\frac{1}{2} \frac{\partial F^{* 2}}{\partial \xi_{k}}\left(L_{\frac{1}{2} F^{2}}(y)\right) \cdot \frac{\partial}{\partial y^{k}}\left(L_{\frac{1}{2} F^{2}}(y)\right)
\end{aligned}
$$


Lembre que $L_{\frac{1}{2} F^{2}}(y)=g_{y}(y, \cdot)=\frac{1}{2} \frac{\partial F^{2}}{\partial y^{i}}(y)$. Então,

$$
\frac{\partial}{\partial y^{k}}\left(L_{\frac{1}{2} F^{2}}(y)\right)=\frac{1}{2} \frac{\partial^{2} F^{2}}{\partial y^{k} \partial y^{i}}(y)=g_{k i}(y) .
$$

Assim,

$$
\frac{1}{2} \frac{\partial F^{2}}{\partial y^{i}}(y)=\frac{1}{2} \frac{\partial F^{* 2}}{\partial \xi_{k}}\left(L_{\frac{1}{2} F^{2}}(y)\right) \cdot g_{k i}(y)
$$

Diferenciando (1.7) em relação a $y^{j}$ temos

$$
\frac{1}{2} \frac{\partial^{2} F^{2}}{\partial y^{i} \partial y^{j}}(y)=\frac{1}{2} \frac{\partial F^{* 2}}{\partial \xi_{k} \xi_{l}}\left(L_{\frac{1}{2} F^{2}}(y)\right) \cdot g_{l j}(y) \cdot g_{k i}(y)+\frac{1}{2} \frac{\partial F^{* 2}}{\partial \xi_{k}}\left(L_{\frac{1}{2} F^{2}}(y)\right) \cdot \frac{\partial g_{k i}(y)}{\partial y^{j}}(y)
$$

Seja $l_{i}(y)$ a i-ésima componente de $L_{\frac{1}{2} F^{2}}(y)$, então,

$$
l_{i}(y)=L_{\frac{1}{2} F^{2}}(y)\left(e_{i}\right)=\frac{1}{2} \frac{\partial F^{2}}{\partial y^{i}}(y)=g_{i j}(y) y^{j}
$$

onde $\left\{e_{1}, \ldots e_{n}\right\}$ é uma base de V. Veja que

$$
\begin{aligned}
\frac{1}{2} \frac{\partial F^{2}}{\partial y^{i}}(y) & =g_{i j}(y) y^{j} \\
\frac{1}{2} \frac{\partial F^{* 2}}{\partial \xi_{k}}\left(L_{\frac{1}{2} F^{2}}(y)\right) \cdot g_{k i}(y) & =g^{* k j}\left(L_{\frac{1}{2} F^{2}}(y)\right) \cdot l_{j}(y) g_{k i}(y) \\
& =g^{* k j}\left(L_{\frac{1}{2} F^{2}}(y)\right) \cdot g_{i j}(y) y^{i} \cdot g_{k i}(y)
\end{aligned}
$$

De (1.7), (1.9) e (1.10) segue que

$$
g_{i j}(y) y^{j}=g^{* k j}\left(L_{\frac{1}{2} F^{2}}(y)\right) \cdot g_{i j}(y) y^{i} \cdot g_{k i}(y)
$$

Multiplicando por $g^{i j}(y)$ de ambos os lados obtemos

$$
y^{j}=g^{* k j}\left(L_{\frac{1}{2} F^{2}}(y)\right) g_{k i}(y) y^{i}=g^{* k j}\left(L_{\frac{1}{2} F^{2}}(y)\right) l_{k}(y)
$$

Então, como $g_{i j}$ é homogênea de grau zero temos

$$
\begin{aligned}
\frac{1}{2} \frac{\partial F^{* 2}}{\partial \xi_{k}}\left(L_{\frac{1}{2} F^{2}}(y)\right) \cdot \frac{\partial g_{k i}(y)}{\partial y^{j}}(y) & =g^{* k m}\left(L_{\frac{1}{2} F^{2}}(y)\right) l_{m}(y) \cdot \frac{\partial g_{k i}(y)}{\partial y^{j}}(y) \\
& =y^{k} \cdot \frac{\partial g_{k i}(y)}{\partial y^{j}}(y) \\
& =0 .
\end{aligned}
$$

Logo, a equação (1.8) fica

$$
g_{i j}(y)=g^{* k l}\left(L_{\frac{1}{2} F^{2}}(y)\right) \cdot g_{l j}(y) \cdot g_{k i}(y)
$$

Aplicando $\left(g^{i j}(y)\right)^{2}$ em ambos os lados da equação acima segue que

$$
g^{i j}(y)=g^{* i j}\left(L_{\frac{1}{2} F^{2}}(y)\right) .
$$




\subsection{Métrica Finsler}

Definição 1.18. Seja $M$ uma variedade diferenciável. Chamamos uma função contínua $F: T M \rightarrow[0,+\infty)$ de métrica Finsler em M se F satisfaz

i. F é suave em $T M \backslash\{0\}$.

ii. $F(\lambda v)=\lambda F(v)$ para todo $v=(x, y) \in T M, \lambda>0$, onde estamos denotando $\lambda v=(x, \lambda y)$.

iii. A forma bilinear simétrica $g_{v}: T_{x} M \times T_{x} M \rightarrow \mathbb{R}$ definida por

$$
g_{v}(u, w):=\left.\frac{1}{2} \frac{\partial^{2}}{\partial s \partial t} F^{2}(x, v+s u+t w)\right|_{s=t=0}, \quad \text { para todo } u, w \in T_{x} M
$$

é positiva definida.

O par $(M, F)$ é chamado Variedade Finsler. $x \in M$.

Note que se $\mathrm{F}$ é uma métrica Finsler em M então $\left.F\right|_{T_{x} M}$ é uma norma de Minkowski em $T_{x} M$ para todo

Definição 1.19. Seja $(M, F)$ uma variedade Finsler. A reversibilidade de F é definida por

$$
r:=\sup \left\{F(-v) ; v \in F^{-1}(1)\right\} .
$$

Dizemos que uma métrica Finsler é reversível ou simétrica se $r=1$, ou seja, se $F(v)=F(-v)$ para todo $v \in V$.

Lema 1.20. Seja $(M, F)$ uma variedade Finsler. Então, $r \geq 1$.

Demonstração. Suponha que para algum $v \in F^{-1}(1)$ temos $F(-v)<1$. Então, existe $\lambda>1$ tal que

$$
1=\lambda F(-v)=F(-\lambda v) \text {. }
$$

Assim, para $u=-\lambda v \in F^{-1}(1)$ temos

$$
F(-u)=F(\lambda v)=\lambda F(v)=\lambda>1 .
$$

Portanto, $r \geq 1$.

Observação 1.21. Podemos ver pelo Lema 1.8, Lema 1.9 e pela Definição 1.19 que uma norma de Minkowski $F$ é uma norma no sentido usual apenas quando $F$ é reversível.

Definição 1.22. Uma co-métrica (ou co-norma) Finsler em uma variedade $\mathrm{M}$ é uma função $H: T^{*} M \rightarrow$ $[0,+\infty)$ tal que

i. H é suave em $T^{*} M \backslash\{0\}$.

ii. $\left.H\right|_{T_{x}^{*} M}: T_{x}^{*} M \rightarrow[0,+\infty)$ é uma norma de Minkowski para todo $x \in M$.

Seja H uma co-norma Finsler em uma variedade M. Então, para cada $x \in M$ temos:

$$
\begin{aligned}
L_{x}: T_{x}^{*} M & \rightarrow T_{x}^{* *} M \\
v & \mapsto D_{2} H(v)
\end{aligned}
$$

onde $D_{2} H(v) w=\left.\frac{d}{d t} H(x, v+t w)\right|_{t=0}$ é a derivada na fibra. O isomorfismo canônico

$$
i: T_{x} M \rightarrow T_{x}^{* *} M
$$


definido da seguinte maneira: Seja $\left\{\frac{\partial}{\partial x^{i}}\right\}$ uma base de $T_{x} M$ induzida por algum sistema de coordenadas e $\left\{d x^{i}\right\}$ a base dual de $\left\{\frac{\partial}{\partial x^{i}}\right\}$, isto é, $\left\{d x^{i}\right\}$ é a base de $T_{x}^{*} M$ satisfazendo $d x^{i}\left(\frac{\partial}{\partial x^{j}}\right)=\delta_{i j}$. Defina

$$
\frac{\partial}{\partial x^{i}}\left(d x^{j}\right):=d x^{j}\left(\frac{\partial}{\partial x^{i}}\right)=\delta_{i j}
$$

Então, $i$ é a transformação linear que aplica

$$
\frac{\partial}{\partial x^{i}} \mapsto \frac{\partial}{\partial x^{i}}(\cdot) \in T^{* *} M
$$

e se estende à $T_{x} M$ por linearidade e tem a propriedade (1.11).

Definição 1.23. Definimos a transformada de Legendre associada à função $H: T^{*} M \rightarrow \mathbb{R}$ como

$$
\begin{aligned}
\mathscr{L}_{H}: T^{*} M & \rightarrow T M \\
\xi & \mapsto i^{-1} \circ L_{\pi(\xi)}(\xi)
\end{aligned}
$$

onde $\pi: T^{*} M \rightarrow M$ é a projeção canônica.

Analogamente,

Definição 1.24. Definimos a transformada de Legendre associada à função $F: T M \rightarrow \mathbb{R}$ como

$$
\begin{aligned}
\mathscr{L}_{F}: T M & \rightarrow T^{*} M \\
y & \mapsto D_{2} F(y)
\end{aligned}
$$

onde $D_{2} F$ é a derivada na direção da fibra.

Teorema 1.25. Sejam H uma co-norma Finsler e $\mathscr{L}_{\frac{1}{2} H^{2}}$ a transformada de Legendre associada à função $\frac{1}{2} H^{2}$. Então:

i. $\mathscr{L}_{\frac{1}{2} H^{2}}:\left(T^{*} M, 0\right) \rightarrow(T M, 0)$ é um homeomorfismo e se restringe a um difeomorfismo em $T^{*} M \backslash\{0\}$.

ii. $F=H \circ \mathscr{L}_{\frac{1}{2} H^{2}}^{-1}$ é uma métrica Finsler em $M$.

Demonstração. Para $x \in M$ fixado seja $\left\{\frac{\partial}{\partial x^{i}}\right\}$ a base de M induzida por algum sistema de coordenadas e sejam $\left\{d x^{i}\right\}$ e $\left\{\frac{\partial}{\partial x^{i}}\right\}(\cdot)$ as bases duais de $T_{x}^{*} M$ e $T_{x}^{* *} M$, respectivamente. Denote por

$$
h^{i j}(\xi)=\frac{1}{2} \frac{\partial^{2} H^{2}}{\partial \xi_{i} \partial \xi_{j}}(\xi), \xi \in T^{*} M \backslash\{0\}
$$

Observe que

$$
L_{x}(\xi)=\frac{1}{2} \frac{\partial H^{2}}{\partial \xi_{i}}(\xi) \frac{\partial}{\partial x^{j}}(\cdot)=h^{i j}(\xi) \xi_{i} \frac{\partial}{\partial x_{j}}(\cdot)
$$

e isto implica que

$$
\mathscr{L}_{\frac{1}{2} H^{2}}(\xi)=i^{-1}\left(h^{i j}(\xi) \xi_{i} \frac{\partial}{\partial x^{j}}(\cdot)\right)=h^{i j}(\xi) \xi_{i} \frac{\partial}{\partial x^{j}}
$$

Logo, $\mathscr{L}_{\frac{1}{2} H^{2}}$ é suave em $T^{*} M \backslash\{0\}$. Pela Proposição $1.15, L_{\frac{1}{2} H^{2}}: T_{x}^{*} M \rightarrow T_{x}^{* *} M$ é uma bijeção. Logo, $\mathscr{L}_{\frac{1}{2} H^{2}}$ é uma bijeção e

$$
\mathscr{L}_{\frac{1}{2} H^{2}}^{-1}(y)=L_{\frac{1}{2} H^{2}}^{-1} \circ i(y) .
$$


Além disso, como o Jacobiano de $\mathscr{L}_{\frac{1}{2}} H^{2}$ é da forma

$$
D \mathscr{L}_{\frac{1}{2} H^{2}}=\left(\begin{array}{ll}
I & 0 \\
* & h^{i j}
\end{array}\right)
$$

então

$$
\operatorname{det}\left(D \mathscr{L}_{\frac{1}{2} H^{2}}\right)=\operatorname{det}\left(h^{i j}\right) \neq 0 .
$$

E pelo Teorema da Função Inversa, $\mathscr{L}_{\frac{1}{2} H^{2}}^{-1}(y)$ é diferenciável para todo $y \in T M \backslash\{0\}$, ou seja, $\mathscr{L}_{\frac{1}{2} H^{2}}(y)$ é um difeomorfismo em $T^{*} M \backslash\{0\} \rightarrow T M \backslash\{0\}$.

Para mostrar (ii) veja que

1. Como H e $\mathscr{L}_{\frac{1}{2} H^{2}}^{-1}$ são $C^{\infty}$ em $T M \backslash\{0\}$ segue que $F=H \circ \mathscr{L}_{\frac{1}{2} H^{2}}^{-1}$ é $C^{\infty}$ em $T M \backslash\{0\}$.

2. Seja $y \in T M$. Como $\mathscr{L}_{\frac{1}{2} H^{2}}$ é uma bijeção existe $\xi \in T^{*} M$ tal que $y=\mathscr{L}_{\frac{1}{2} H^{2}}(\xi)$. Além disso, como $\mathscr{L}_{\frac{1}{2} H^{2}}$ é homogênea de grau 1 temos

$$
\mathscr{L}_{\frac{1}{2} H^{2}}^{-1}(\lambda y)=\mathscr{L}_{\frac{1}{2} H^{2}}^{-1}\left(\mathscr{L}_{\frac{1}{2} H^{2}}(\lambda \xi)\right)=\lambda \xi=\lambda \mathscr{L}_{\frac{1}{2} H^{2}}^{-1}(y)
$$

Logo,

$$
F(\lambda y)=H \circ \mathscr{L}_{\frac{1}{2} H^{2}}^{-1}(\lambda y)=H\left(\lambda \mathscr{L}_{\frac{1}{2} H^{2}}^{-1}(y)\right)=\lambda H \circ \mathscr{L}_{\frac{1}{2} H^{2}}^{-1}(y)=\lambda F(y),
$$

isto é, F é homogênea de grau 1.

3. Sejam $w^{i}$ as coordendas para $T_{x}^{* *}$, então,

$$
\begin{aligned}
g_{i j}(y) & =\frac{1}{2} \frac{\partial^{2} F^{2}}{\partial y^{i} \partial y^{j}} \\
& =\frac{1}{2} \frac{\partial^{2} H^{2}\left(\mathscr{L}_{\frac{1}{2} H^{2}}^{-1}(y)\right)}{\partial y^{i} \partial y^{j}} \\
& =\frac{1}{2} \frac{\partial^{2}\left(H^{2} \circ L_{x}^{-1} \circ i\right)}{\partial y^{i} \partial y^{j}}(y) \\
& =\frac{1}{2} \frac{\partial^{2} H^{2} \circ L_{x}^{-1}}{\partial w^{i} \partial w^{j}}(i(y))
\end{aligned}
$$

Pela Proposição 1.15, dado $\eta \in T^{* *} M$, então, $L_{x}(\lambda \xi)=\eta$ onde $H(\xi)=1 \mathrm{e}$

$$
\lambda=H^{*}(\eta)=\max \left\{\eta(\xi) ; \xi \in T_{x}^{*} M \text { e } H(\xi)=1\right\} .
$$

Assim,

$$
H \circ L_{x}^{-1}(\eta)=H(\lambda \xi)=\lambda H(\xi)=\lambda=H^{*}(\eta) .
$$

Logo, denotando por $h_{i j}$ a inversa de $h^{i j}$, pela Proposição 1.17 segue que

$$
\begin{aligned}
h_{i j}(\xi) & =h^{* i j} \circ L_{x}(\xi) \\
& =\frac{1}{2} \frac{\partial^{2} H^{2} \circ L_{x}^{-1}}{\partial w^{i} \partial w^{j}} \circ L_{x}(\xi) \\
& =\frac{1}{2} \frac{\partial^{2} H^{2} \circ L_{x}^{-1}}{\partial w^{i} \partial w^{j}}\left(i\left(i^{-1} L_{x}(\xi)\right)\right) \\
& =g_{i j}\left(\mathscr{L}_{\frac{1}{2} H^{2}}(\xi)\right)
\end{aligned}
$$

isto é,

$$
g_{i j}(y)=h_{i j} \circ \mathscr{L}_{\frac{1}{2} H^{2}}^{-1}(y), y \in T M \backslash\{0\}
$$


Como uma matriz é positiva definida se, e somente se, seus autovalores são todos positivos e se $\mu \neq 0$ é um autovalor de uma matriz, então, $\frac{1}{\mu}$ é um autovalor de sua inversa segue que

$$
h^{i j} \text { positiva definida } \Leftrightarrow h_{i j} \text { é positiva definida . }
$$

Logo, $g_{i j}(y)$ é positiva definida.

Portanto, F é uma métrica Finsler.

Teorema 1.26. Sejam F uma métrica Finsler e $\mathscr{L}_{\frac{1}{2} F^{2}}$ a transformada de Legendre associada à função $\frac{1}{2} F^{2}$. Então:

i. $\mathscr{L}_{\frac{1}{2} F^{2}}:(T M, 0) \rightarrow\left(T^{*} M, 0\right)$ é um homeomorfismo e se restringe a um difeomorfismo em $T M \backslash\{0\}$.

ii. $H=F \circ \mathscr{L}_{\frac{1}{2} F^{2}}^{-1}$ é uma co-norma Finsler em $M$.

Demonstração. Análoga a demonstração do Teorema (1.25).

Teorema 1.27. Sejam H uma co-norma Finsler, $\mathscr{L}_{\frac{1}{2} H^{2}}$ a transformada de Legendre associada à função $\frac{1}{2} H^{2}$ e $\mathscr{L}_{\frac{1}{2} F^{2}}$ a transformada de Legendre associada à função $\frac{1}{2} F^{2}$, onde $F=H \circ \mathscr{L}_{\frac{1}{2} H^{2}}^{-1}$. Então,

$$
\mathscr{L}_{\frac{1}{2} F^{2}}=\mathscr{L}_{\frac{1}{2} H^{2}}^{-1}
$$

Demonstração. Observe que

$$
\mathscr{L}_{\frac{1}{2} F^{2}}(y)=\frac{1}{2} \frac{\partial F^{2}}{\partial y^{i}}(y)=g_{i j} y^{i} .
$$

Seja $\left(h^{i j}\right)$ como em (1.12) e $\left(h_{i j}\right)$ a sua inversa. Então,

$$
L_{x}^{-1}(\eta)=h_{i j}(\xi) \eta_{i}=h^{* i j}(\eta) \eta_{i}, \quad \eta=h^{i j}(\xi) \xi_{i}=L_{x}(\xi)
$$

onde na última igualdade usamos a Proposição (1.17). Assim, por (1.16), (1.14) e (1.13) segue que

$$
\begin{aligned}
\mathscr{L}_{\frac{1}{2} H^{2}}^{-1} & =L_{x}^{-1} \circ i(x) \\
& =h^{* i j}(i(y))(i \circ y)^{i} \\
& =\frac{1}{2} \frac{\partial^{2} H^{2} \circ L_{x}^{-1}}{\partial w^{i} \partial w^{j}}(i(y))(i \circ y)^{i} \\
& =g_{i j}(y) y^{i}
\end{aligned}
$$

Portanto,

$$
\mathscr{L}_{\frac{1}{2} F^{2}}=\mathscr{L}_{\frac{1}{2} H^{2}}^{-1}
$$

A seguir são apresentados mais alguns exemplos de métricas Finsler e co-norma Finsler.

\subsubsection{Métricas Riemannianas}

Seja M uma variedade diferenciável. Uma métrica Riemanniana $g$ em M é uma família $g=\left\{g_{x}\right\}_{x \in M}$, onde para todo $x \in M, g_{x}$ é uma forma bilinear simétrica e positiva definida em $T_{x} M$ tal que em coordenadas locais $\left(x^{i}\right)$

$$
g_{i j(x)}=g_{x}\left(\left.\frac{\partial}{\partial x^{i}}\right|_{x},\left.\frac{\partial}{\partial x^{j}}\right|_{x}\right)
$$


são funções $C^{\infty}$. Também podemos escrever

$$
g_{x}=g_{i j(x)} d x^{i} \otimes d x^{j} .
$$

Seja

$$
F_{x}(y)=\sqrt{g_{x}(y, y)}, \quad y \in T_{x} M .
$$

A família de normas $F=\left\{F_{x}\right\}_{x \in M}$ é uma métrica Finsler em M. Uma métrica Finsler é chamada de Riemanniana se pode ser expressa por (1.17) para alguma métrica Riemanniana g. Observe que nesse caso a forma bilinear simétrica associada à $\mathrm{F}$ como na Definição 1.18 não depende de $y$ pois

$$
\begin{aligned}
g_{i j}(y) & :=\frac{1}{2} \frac{\partial^{2} F_{x}^{2}}{\partial y^{i} \partial y^{j}}(y) \\
& =\frac{\partial F_{x}}{\partial y^{i}}(y) \frac{\partial F_{x}}{\partial y^{j}}(y)+F_{x}(y) \frac{\partial^{2} F_{x}}{\partial y^{i} \partial y^{j}}(y) \\
& =\left(\frac{g_{i j(x)} y^{j}}{F_{x}(y)}\right)\left(\frac{g_{i j(x)} y^{i}}{F_{x}(y)}\right)+F_{x}(y)\left(\frac{g_{i j(x)}}{F_{x}(y)}-\frac{g_{i j(x)} y^{j}}{F_{x}(y)^{2}} \frac{g_{i j(x)} y^{i}}{F_{x}(y)}\right) \\
& =\left(\frac{g_{i j(x)} y^{j}}{F_{x}(y)}\right)\left(\frac{g_{i j(x)} y^{i}}{F_{x}(y)}\right)+g_{i j(x)}-\left(\frac{g_{i j(x)} y^{j}}{F_{x}(y)}\right)\left(\frac{g_{i j(x)} y^{i}}{F_{x}(y)}\right) \\
& =g_{i j(x)}
\end{aligned}
$$

Este é o exemplo mais simples de uma métrica Finsler reversível.

\subsubsection{Métrica dual}

Sejam $(M, F)$ uma variedade Finsler e $F^{*}$ a norma dual de $F$. Como anteriormente, denote

$$
g_{i j}(y):=\frac{1}{2} \frac{\partial^{2} F^{2}}{\partial y^{i} \partial y^{j}}(x, y) \quad \text { e } \quad g^{* i j}(\xi):=\frac{1}{2} \frac{\partial^{2} F^{* 2}}{\partial \xi_{i} \partial \xi_{j}}(x, \xi)
$$

onde $y \in T_{x} M \backslash\{0\}$ e $\xi \in T_{x}^{*} M \backslash\{0\}$. Se $A^{i j}(y)$ é a inversa de $g_{i j}(y)$ e $\xi_{i}=g_{i j}(y) y^{j}$ então pela Observação 1.14 e Proposição 1.17 temos

$$
A^{i j}\left(y^{i}\right)=g^{* i j}\left(g_{i j}(y) y^{j}\right)=g^{* i j}\left(\xi^{i}\right) .
$$

Assim, $g_{i j}(y) \cdot g^{* j k}(\xi)=\delta_{i k}$ e por esse motivo, em alguns momentos, iremos usar a notação

$$
g^{* i j}(\xi):=g^{i j}(\xi)
$$

para nos referirmos a matriz inversa de $g_{i j}(y)$, onde $\xi_{i}=g_{i j}(y) y^{j}$. Pela Proposição 1.16 segue que

$$
F^{*}=F \circ \mathscr{L}_{\frac{1}{2} F^{2}}
$$

e, portanto, pelo Teorema 1.26 concluímos que $F^{*}$ é uma co-norma Finsler. Assim, $g^{i j}(\xi)$ é positivamente homogêneo de grau 0 e pelo Teorema 1.3 obtemos as identidades

$$
\frac{\partial g^{i j}}{\partial \xi_{k}}(\xi) \xi^{i}=\frac{\partial g^{i j}}{\partial \xi_{k}}(\xi) \xi^{j}=\frac{\partial g^{i j}}{\partial \xi_{k}}(\xi) \xi^{k}=0
$$

Observe que pela Proposição 1.15 para todo $\xi \in T^{*} M$ existe $v \in T_{x} M$ com $F(x, v)=1$ tal que $\xi=$ $g_{v}(\lambda v, \cdot)$, onde $\lambda=F^{*}(x, \xi)$. Assim, se $N: T^{*} M \rightarrow[0,+\infty)$ é uma função definida por

$$
N^{2}(x, \xi)=g^{i j}(\xi) \xi_{i} \xi_{j}
$$


segue que

$$
\begin{aligned}
N^{2}(x, \xi) & =g^{i j}(\xi) \xi_{i} \xi_{j} \\
& =g^{i j}(\xi) \cdot \lambda g_{i j}(v) v_{j} \cdot \lambda g_{i j}(v) v_{i} \\
& =\lambda^{2} g_{i j}(v) v_{i} v_{j} \\
& =\lambda^{2} F^{2}(x, v) \\
& =\lambda^{2} \\
& =F^{* 2}(x, \xi)
\end{aligned}
$$

$\operatorname{Logo}, N=F^{*}$.

\subsubsection{Métricas do tipo Randers}

Sejam $g$ uma métrica Riemannina e $\beta$ uma 1-forma em M. Denote por

$$
\|\beta\|_{g}=\sup \{\beta(v) ; g(v, v)=1, v \in T M\}
$$

e $\alpha_{x}(u):=\sqrt{g_{x}(u, u)}$, onde $u \in T_{x} M$. Seja $\left(a_{i j}\right)$ a matriz simétrica e positiva definida associada a métrica Riemanniana $g$.

Observação 1.28. Como vimos na seção 1.2.2

$$
\|\beta\|_{g}=\sqrt{a^{i j} b_{i} b_{j}}
$$

onde $\left(a^{i j}\right)=\left(a_{i j}\right)^{-1}$ pois $\|\cdot\|_{g}$ é a métrica dual da métrica Riemanniana $g$. Essa expressão para $\|\beta\|_{g}$, em geral, é mais útil.

Proposição 1.29. Seja $F: T M \rightarrow \mathbb{R}$ definida por $F(x, y)=\alpha_{x}(y)+\beta_{x}(y)$. Fixado $x \in M$ então $F(x, y)>0$ para todo $y \in T_{x} M \backslash\{0\}$ se, e somente se, $\|\beta\|_{g}<1$.

Demonstração. Em coordenadas locais, temos

$$
F(x, y)=\sqrt{a_{i j} y^{i} y^{j}}+b_{i} y^{i}
$$

Observe que $F(x, y)>0$ significa que

$$
\sqrt{a_{i j} y^{i} y^{j}}>-b_{i} y^{i}
$$

Suponha que $F(x, y)>0$, isto é, vale (1.21) para todo $y \in T_{x} M \backslash\{0\}$. Queremos mostrar que $\|\beta\|_{g}<1$. Veja que se $\beta=0$ obtemos o desejado trivialmente. Assim, podemos supor, sem perda de generalidade, que $\beta \neq 0$. Tomando $y_{i}=-a^{i j} b_{j}$ segue-se que

$$
\begin{aligned}
\sqrt{a_{i j}\left(-a^{i j} b_{j}\right)\left(-a^{i j} b_{i}\right)} & >-b_{i}\left(-a^{i j} b_{j}\right) \\
b_{i} a^{i j} b_{j} & <\sqrt{b_{j} a^{i j} b_{i}} \\
\frac{b_{i} a^{i j} b_{j}}{\sqrt{b_{j} a^{i j} b_{i}}} & <1
\end{aligned}
$$

Logo, $\|\beta\|_{g}=\sqrt{b_{j} a^{i j} b_{i}}<1$. Agora suponha que $\|\beta\|_{g}=\sqrt{b_{j} a^{i j} b_{i}}<1$. Então, pela desigualdade de 
Cauchy-Schwarz temos para todo $y \neq 0$

$$
\begin{aligned}
\left(b_{i} y^{i}\right)^{2} & =\left[a_{i j}\left(a^{i j} b_{i}\right) y^{i}\right]^{2} \\
& \leq\left[a_{i j}\left(a^{i j} b_{i}\right)\left(a^{i j} b_{j}\right)\right] \cdot\left[a_{i j} y^{i} y^{j}\right] \\
& =\left[b_{i} a^{i j} b_{j}\right] \cdot\left[a_{i j} y^{i} y^{j}\right] \\
& <a_{i j} y^{i} y^{j}
\end{aligned}
$$

Logo, $\left|b_{i} y^{i}\right|<\sqrt{a_{i j} y^{i} y^{j}}$ para todo $y \neq 0$ e, portanto, vale (1.21).

Teorema 1.30. A função $F(x, y)=\alpha_{x}(y)+\beta_{x}(y)$ é uma métrica Finsler em $M$ se, e somente se, $\|\beta\|_{g}<1$.

Demonstração. Claramente F é suave em $T M \backslash\{0\}$ e positivamente homogênea de grau 1. Então, falta apenas verificar que F satisfaz a condição (iii) da Definição 1.18 se, e somente se, $\|\beta\|_{g}<1$. Em coordenadas locais, temos

$$
F(x, y)=\sqrt{a_{i j} y^{i} y^{j}}+b_{i} y^{i}
$$

Então,

$$
\begin{aligned}
g_{i j}(y) & =\frac{1}{2} \frac{\partial^{2} F^{2}}{\partial y^{i} \partial y^{j}}(x, y) \\
& =F_{y^{i}}(x, y) F_{y^{j}}(x, y)+F(x, y) F_{y^{i} y^{j}}(x, y) \\
& =\left(\frac{a_{i j} y^{j}}{\alpha_{x}}+b_{i}\right)\left(\frac{a_{i j} y^{i}}{\alpha_{x}}+b_{j}\right)+\frac{F}{\alpha_{x}}\left(a_{i j}-\frac{a_{i j} y^{i}}{\alpha_{x}} \frac{a_{i j} y^{j}}{\alpha_{x}}\right)
\end{aligned}
$$

Por ([BCS00], §11.2) obtemos seguinte igualdade:

$$
\operatorname{det}\left(g_{i j}\right)=\left(\frac{F}{\alpha_{x}}\right)^{n+1} \operatorname{det}\left(a_{i j}\right)
$$

Seja

$$
F_{\varepsilon}:=\sqrt{a_{i j} y^{i} y^{j}}+\varepsilon b_{i} y^{i}
$$

onde $\|\beta\|_{g}<1$ e $0 \leq \varepsilon \leq 1$. Pela Proposição 1.29 segue que $F_{\varepsilon}$ é positiva em $T M \backslash\{0\}$. Seja $g_{\varepsilon}$ a abreviação da forma bilinear associada a função $F_{\mathcal{\varepsilon}}$. Por (1.22) temos

$$
\operatorname{det}\left(g_{\varepsilon}\right)=\left(\frac{F_{\varepsilon}}{\alpha_{x}}\right)^{n+1} \operatorname{det}\left(a_{i j}\right) .
$$

Assim, $\operatorname{det}\left(g_{\varepsilon}\right)$ é sempre positivo. Em particular, nenhum dos auto-valores de $g_{\varepsilon}$ é zero.

Os auto-valores de $g_{\varepsilon}$ dependem continuamente de $\varepsilon$. Em $\varepsilon=0$, eles são simplesmente os de $\left(a_{i j}\right)$ e, portanto, são todos positivos. Como $\varepsilon$ varia entre $0 \mathrm{e} 1$, nenhum dos auto-valores $\lambda_{\varepsilon}$ de $g_{\varepsilon}$ pode se tornar negativo, pois se $\lambda_{\varepsilon}<0$ então existe $0<\varepsilon^{\prime}<\varepsilon$ tal que $\lambda_{\mathcal{\varepsilon}^{\prime}}=0$, mas isso não ocorre. Logo, todos os autovalores de $g_{\varepsilon}$ permanecem positivos. Fazendo $\varepsilon=1$ segue que os auto-valores de $\left(g_{i j}\right)$ são todos positivos se $\|\beta\|_{g}<1$. Portanto, $\left(g_{i j}\right)$ é positiva definida se, e somente se, $\|\beta\|_{g}<1$.

Definição 1.31. Uma métrica Finsler como no Teorema 1.30 é chamada de métrica Finsler do tipo Randers. Usamos também a notação $(M, g, \beta)$ para a variedade Finsler $(M, F)$.

\subsection{Geodésicas}

Seja $(M, F)$ uma variedade Finsler e $\gamma:[a, b] \rightarrow M$ uma curva $C^{\infty}$ por partes com velocidade constante, isto é, $F(\dot{\gamma}(t))=\lambda=$ constante para todo $t \in[a, b]$. 
Definição 1.32. A curva $\gamma:[a, b] \rightarrow M$ é uma geodésica se, e somente se, satisfaz

$$
\ddot{\gamma}(t)+2 G^{i}(\gamma(t), \dot{\gamma}(t))=0 \quad \text { para todo } i=1, \ldots, n
$$

onde

$$
G^{i}(x, y)=\frac{1}{4} g^{i l}\left(2 \partial_{x^{k}} g_{r l}-\partial_{x^{l}} g_{r k}\right) y^{r} y^{k}
$$

$\mathrm{e}\left(g^{j l}\right)=\left(g_{j l}\right)^{-1}$

Para mais detalhes ver Seção 1.4 de [SH13] e Capítulo 5 de [She01].

Queremos transformar o sistema de segunda ordem (1.23) em um sistema de primeira ordem no fibrado cotangente $T^{*} M$. Seja $\left(x^{1}, \ldots, x^{n}, \xi_{1}, \ldots, \xi_{n}\right)$ as coordenadas locais de $T^{*} M$ e denote por

$$
H(x, \xi)=\frac{1}{2} g^{i j}(x, \xi) \xi_{i} \xi_{j} .
$$

onde $g^{i j}(x, \xi)$ é como na equação (1.18), isto é, $g_{i j}(x, \dot{x}) \cdot g^{j k}(x, \xi)=\delta_{i k}$ e $\xi_{j}=g_{i j}(x, \dot{x}) \dot{x}^{i}$.

Teorema 1.33. A equação $\ddot{x}(t)+2 G^{i}(x(t), \dot{x}(t))=0$ para todo $i=1, \ldots, n$ é equivalente ao seguinte sistema em $T^{*} M$ :

$$
\begin{aligned}
& \dot{x}^{i}=\frac{\partial H}{\partial \xi_{i}}=g^{i j}(x, \xi) \xi_{j} \\
& \dot{\xi}_{i}=-\frac{\partial H}{\partial x^{i}}=-\frac{1}{2} \partial_{x^{i}}\left(g^{j k}(x, \xi)\right) \xi_{j} \xi_{k}
\end{aligned}
$$

Demonstração. Da primeira equação em (1.26) e de (1.19) temos

$$
\begin{aligned}
\ddot{x}^{i} & =g^{i j}(x, \xi) \dot{\xi}_{j}+\partial_{x^{k}}\left(g^{i j}(x, \xi)\right) \dot{x}^{k} \xi_{j}+\partial_{\xi^{k}}\left(g^{i j}(x, \xi)\right) \xi_{j} \dot{\xi}^{k} \\
& =g^{i j}(x, \xi) \dot{\xi}_{j}+\partial_{x^{k}}\left(g^{i j}(x, \xi)\right) \dot{x}^{k} g_{j l}(x, \dot{x}) \dot{x}^{l}
\end{aligned}
$$

E usando a segunda equação temos

$$
\begin{aligned}
\ddot{x}^{i} & =-\frac{1}{2} g^{i j} \partial_{x^{j}}\left(g^{l k}\right) \xi_{l} \xi_{k}+\partial_{x^{k}}\left(g^{i j}\right) g_{j l} \dot{x}^{k} \dot{x}^{l} \\
& =\frac{1}{2} g^{i j} g^{l m} \partial_{x^{j}}\left(g_{m n}\right) g^{n k} g_{l r} \dot{x}^{r} g_{k s} \dot{x}^{s}-g^{i m} \partial_{x^{k}}\left(g^{m n}\right) g^{n j} g_{j l} \dot{x}^{k} \dot{x}^{l}
\end{aligned}
$$

onde usamos que $\partial_{x^{l}}\left(g^{i j}\right)=-g^{i m} \partial_{x^{l}}\left(g_{m n}\right) g^{n j}$ pois $g^{i j} g_{j k}=\delta_{i k}$. Então,

$$
\begin{aligned}
\ddot{x} & =\frac{1}{2} g^{i j} \partial_{x^{j}}\left(g_{m n}\right) \dot{x}^{m} \dot{x}^{n}-g^{i m} \partial_{x^{k}}\left(g^{m n}\right) \dot{x}^{k} \dot{x}^{n} \\
& =-\frac{1}{2} g^{i j}\left(-\partial_{x^{j}}\left(g_{m n}\right)-2 \partial_{x^{k}}\left(g^{m n}\right)\right) \dot{x}^{m} \dot{x}^{n} \\
& =-2 G^{i}(x, \dot{x})
\end{aligned}
$$

Portanto, os sistemas são equivalentes.

Definição 1.34. O fluxo determinado por (1.26) é chamado de fluxo cogeodésico. O fluxo geodésico em TM é obtido do fluxo cogeodésico pela primeira equação de (1.26).

Definição 1.35. Seja $(M, F)$ uma variedade Finsler. Uma curva $\gamma$ é uma geodésica periódica com período $r$ (onde $r$ é um número real não nulo) se, e somente se: 
(i) $\gamma$ é uma geodésica

(ii) $\gamma$ é periódica como uma aplicação de $\mathbb{R}^{+}$em $M$ (parametrizada de forma que $\left.F(\gamma(t), \dot{\gamma}(t))=1\right)$ com período $r$. O número $r$ é o comprimento da geodésica periódica.

Notação 1.36. Seja $(M, g)$ uma variedade Riemanniana. Chamaremos o conjunto dos vetores unitários de $T M$ de fibrado tangente unitário e o denotaremos por $T_{1} M$, isto é,

$$
T_{1} M=\left\{(x, v) \in T M ; g_{x}(v, v)=1\right\} .
$$

Definição 1.37. Uma variedade $M$ é uma $C_{r}$-variedade se existe uma métrica Riemanniana $g$ em $M$ tal que todas as geodésicas são periódicas com mesmo comprimento $r$. Diz-se também que $g$ é uma $C_{r}$-métrica.

Observação 1.38. Se $M$ é uma $C_{r}$-variedade então para cada $\eta$ no fibrado tangente unitário de $\mathrm{M}$ a curva integral $s \mapsto \phi_{s}(\eta)$ do fluxo geodésico no fibrado tangente unitário $T_{1} M$ é periódica com período $r$ e $\phi_{s}(\eta)=$ $\eta$ se, e somente se, $s$ é um múltiplo de $r$.

Definição 1.39. Uma ação de grupo $G \times X \rightarrow X$ é chamada de livre se para todo $x \in X, g x=x$ implica que $g=I$, isto é, somente o elemento identidade fixa qualquer $x$.

Seja $M$ uma $C_{r}$-variedade. O campo de vetores geodésico gera uma ação livre de $S^{1}=\mathbb{R} / r \mathbb{Z}$ no fibrado tangente unitário como descreveremos a seguir: Dado $(x, v) \in T_{1} M$, seja $\gamma_{x}^{v}(t)$ a geodésica em $M$ tal que

$$
\gamma_{x}^{v}(0)=x \quad \text { e } \quad \dot{\gamma}_{x}^{v}(0)=v .
$$

Então, defina a ação de $S^{1}$ em $T_{1} M$ por

$$
s \cdot(x, v):=\left(\gamma_{x}^{v}(s), \dot{\gamma}_{x}^{v}(s)\right) \quad \text { para todo } s \in S^{1}=\mathbb{R} / r \mathbb{Z} .
$$

Isso induz uma relação de equivalência onde

$$
\left(x_{1}, v_{1}\right) \sim\left(x_{2}, v_{2}\right)
$$

se, e somente se, existe $s \in S^{1}$ tal que

$$
\left(x_{2}, v_{2}\right)=\left(\gamma_{x_{1}}^{v_{1}}(s), \dot{\gamma}_{x_{1}}^{\nu_{1}}(s)\right) .
$$

Fazendo o quociente de $T_{1} M$ pela ação de $S^{1}$ (ou melhor, pela relação de equivalência) obtemos

$$
C:=\frac{T_{1} M}{S^{1}}=\left\{[(x, v)] ;(x, v) \in T_{1} M\right\} .
$$

Observação 1.40. O espaço quociente $C=T_{1} M / S^{1}$ é uma variedade de dimensão $2 n-2$ (a variedade das geodésicas orientadas). Podemos considerar também a ação livre de $\mathbb{Z}_{2} \times S^{1}$ em $T_{1} M$ para obtermos outra variedade, a variedade de geodésicas não orientadas, da qual $C$ é uma dupla cobertura.

Observação 1.41. Se pedirmos somente que o fluxo geodésico seja periódico, com período $r$, isto é, as geodésicas periódicas não tiverem necessariamente o mesmo comprimento então a ação de $S^{1}$ não será livre e não podemos definir a variedade de geodésicas.

\subsection{Derivada Covariante}

Para um campo de vetores $X=\left(X^{1}, \ldots, X^{n}\right)$ em um aberto $U \subset \mathbb{R}^{n}$, a derivada direcional $D_{v} X$ na direção $v \in T_{x} \mathbb{R}^{n}=\mathbb{R}^{n}$ é definida por

$$
D_{v} X:=\left(d X^{1}(v), \ldots, d X^{n}(v)\right)=v^{i} \frac{\partial X}{\partial x^{i}} .
$$

Podemos estender a noção de derivada direcional para campos de vetores em um espaço Finsler. 
Definição 1.42. Seja $(M, F)$ uma variedade Finsler. Em cada $x \in M$ defina

$$
D: T_{x} M \times C^{\infty}(T M) \rightarrow T_{x} M
$$

por

$$
D_{y} U:=\left.\left\{d U^{i}(y)+U^{j}(x) N_{j}^{i}(y)\right\} \frac{\partial}{\partial x^{i}}\right|_{x}
$$

onde $y \in T_{x} M, U \in C^{\infty}(T M)$ e $N_{j}^{i}(y)$ são funções locais em $T M$ tais que

$$
N_{j}^{i}(y):=\frac{\partial G^{i}}{\partial y^{j}}(y)=\frac{\partial}{\partial y^{j}}\left[\frac{1}{4} g^{i l}(y)\left\{2 \frac{\partial g_{j l}}{\partial x^{k}}(y)-\frac{\partial g_{j k}}{\partial x^{l}}(y)\right\} y^{j} y^{k}\right] .
$$

Chamamos $D_{y} U(x)$ a derivada covariante de $U$ em $x$ na direção de $y$.

A derivada covariante $D$ tem as seguintes propriedades:

(a) $D_{y}(U+V)=D_{y} U+D_{y} V$;

(b) $D_{y}(f U)=d f_{x}(y)+f(x) D_{y} U$;

(c) $D_{\lambda y} U=\lambda D_{y} U, \lambda>0$.

A família $D:=\left\{D_{y}\right\}_{y \in T M}$ é chamada a conexão de $F$. Se em adição, $D$ é linear, isto é,

(d) $D_{y+v} U=D_{y} U+D_{v} U$

então $D$ é chamada uma conexão afim em $T M$ (ou $M$ ). Chamamos essa conexão de Levi-Civita.

\subsection{Campos de Jacobi}

Definição 1.43. Seja $(M, F)$ uma variedade Finsler. Considere uma geodésica $c(t), a \leq t \leq b$. Uma aplicação $C^{\infty}, H:(-\varepsilon, \varepsilon) \times[a, b] \rightarrow M$ é chamada uma variação da geodésica $c$ se

$$
H(0, t)=c(t)
$$

e para cada $s \in(-\varepsilon, \varepsilon)$, a curva

$$
c_{s}(t):=H(s, t)
$$

é uma geodésica.

Lema 1.44. Seja $(M, F)$ uma variedade Finsler. Existe uma família de transformações $R_{y}: T_{x} M \rightarrow T_{x} M$, $y \in T_{x} M \backslash\{0\}$, tais que para qualquer variação de geodésicas $H$ da geodésica c, o campo de vetores

$$
J(t):=\frac{\partial H}{\partial s}(0, t)
$$

ao longo de c satisfaz a seguinte equação:

$$
D_{\dot{c}} D_{\dot{c}} J+R_{\dot{c}}(J)=0 .
$$

Demonstração. Assuma que cada $c_{s}(t)=H(s, t)$ é uma geodésica. Assim,

$$
\frac{\partial^{2} H^{i}}{\partial t^{2}}+2 G^{i}\left(H, \frac{\partial H}{\partial t}\right)=0 .
$$

Seja

$$
T=T^{i} \frac{\partial}{\partial x^{i}}:=\frac{\partial H}{\partial t}, \quad U=U^{i} \frac{\partial}{\partial x^{i}}:=\frac{\partial H}{\partial s} .
$$


Então, a equação (1.31) com a notação acima se torna

$$
\frac{\partial T^{i}}{\partial t}+2 G^{i}(H, T)=0
$$

Note que

$$
\frac{\partial T^{i}}{\partial s}=\frac{\partial}{\partial s}\left(\frac{\partial H^{i}}{\partial t}\right)=\frac{\partial}{\partial t}\left(\frac{\partial H^{i}}{\partial s}\right)=\frac{\partial U^{i}}{\partial t}
$$

Diferenciando (1.32) com respeito a $s$ temos

$$
\frac{\partial^{2} T^{i}}{\partial s \partial t}=-2 U^{k} \frac{\partial G^{i}}{\partial x^{k}}(H, T)-2 \frac{\partial T^{j}}{\partial s} \frac{\partial G^{i}}{\partial y^{j}}(H, T)
$$

ou seja,

$$
\frac{\partial^{2} U^{i}}{\partial t^{2}}=-2 U^{k} \frac{\partial G^{i}}{\partial x^{k}}(H, T)-2 \frac{\partial U^{j}}{\partial t} \frac{\partial G^{i}}{\partial y^{j}}(H, T)
$$

Observe ainda que

$$
\begin{aligned}
\frac{\partial}{\partial s}\left[G^{i}(H, T)\right] & =U^{k} \frac{\partial G^{i}}{\partial x^{k}}(H, T)+\frac{\partial T^{j}}{\partial s} \frac{\partial G^{i}}{\partial y^{j}}(H, T) \\
& =U^{k} \frac{\partial G^{i}}{\partial x^{k}}(H, T)+\frac{\partial U^{j}}{\partial t} \frac{\partial G^{i}}{\partial y^{j}}(H, T)
\end{aligned}
$$

$\mathrm{e}$

$$
\begin{aligned}
\frac{\partial}{\partial t}\left[\frac{\partial G^{i}}{\partial y^{j}}(H, T)\right] & =T^{k} \frac{\partial^{2} G^{i}}{\partial x^{k} \partial y^{j}}(H, T)+\frac{\partial T^{k}}{\partial t} \frac{\partial^{2} G^{i}}{\partial x^{k} \partial y^{j}}(H, T) \\
& =T^{k} \frac{\partial^{2} G^{i}}{\partial x^{k} \partial y^{j}}(H, T)-2 G^{k}(H, T) \frac{\partial^{2} G^{i}}{\partial x^{k} \partial y^{j}}(H, T)
\end{aligned}
$$

onde usamos (1.32) na última igualdade. Pelas igualdades acima obtemos

$$
\begin{aligned}
D_{T} D_{T} U & =D_{T}\left[\left(\frac{\partial U^{i}}{\partial t}+U^{j} \frac{\partial G^{i}}{\partial y^{j}}(H, T)\right) \frac{\partial}{\partial x^{i}}\right] \\
& =-U^{k}\left\{2 \frac{\partial G^{i}}{\partial x^{k}}-y^{j} \frac{\partial^{2} G^{i}}{\partial x^{j} \partial y^{k}}+2 G^{j} \frac{\partial^{2} G^{i}}{\partial y^{j} \partial y^{k}}-\frac{\partial G^{i}}{\partial y^{j}} \frac{\partial G^{j}}{\partial y^{k}}\right\} \frac{\partial}{\partial x^{i}} \\
& =-U^{k} R_{k}^{i}(T) \frac{\partial}{\partial x^{i}}
\end{aligned}
$$

onde

$$
R_{k}^{i}(y):=2 \frac{\partial G^{i}}{\partial x^{k}}-y^{j} \frac{\partial^{2} G^{i}}{\partial x^{j} \partial y^{k}}+2 G^{j} \frac{\partial^{2} G^{i}}{\partial y^{j} \partial y^{k}}-\frac{\partial G^{i}}{\partial y^{j}} \frac{\partial G^{j}}{\partial y^{k}} .
$$

Para cada $y \in T_{x} M \backslash\{0\}$, defina uma transformação linear

$$
R_{y}=\left.R_{k}^{i}(y) \frac{\partial}{\partial x^{i}} \otimes d x^{k}\right|_{x}: T_{x} M \rightarrow T_{x} M
$$

Assim,

$$
D_{T} D_{T} U+R_{T}(U)=0 .
$$

Restringindo a equação acima para $c$ e fazendo $J(t):=U(0, t)$ obtemos

$$
D_{\dot{c}} D_{\dot{c}} J+R_{\dot{c}}(J)=0 .
$$


A variação de geodésicas dá origem a uma família de transformações

$$
R=\left\{R_{y}: T_{x} M \rightarrow T_{x} M ; y \in T_{x} \backslash\{0\}, x \in M\right\} .
$$

\section{Chamamos $R$ de curvatura de Riemann.}

Definição 1.45. Seja $c(t)$ uma geodésica não-constante de $F$. Um campo de Jacobi ao longo de $c$ é um campo de vetores $J(t)$ ao longo de $c$ que satisfaz a EDO linear

$$
D_{\dot{c}} D_{\dot{c}} J+R_{\dot{c}}(J)=0,
$$

chamada de equação de Jacobi.

Sejam $p=c(a)$ e $q=c(b)$ dois pontos na geodésica $c, \operatorname{com} a \neq b$.

Definição 1.46. Os pontos $p$ e $q$ são conjugados ao longo de $c$ se existe um campo de Jacobi não-nulo ao longo de $c$ tal que $J(a)=J(b)=0$. A multiplicidade de $p$ e $q$ como pontos conjugados é igual a dimensão do espaço vetorial de todos os campos de Jacobi que se anulam em $t=a$ e $t=b$. 


\section{Capítulo 2}

\section{Espaços Projetivos e Grupos de Isometrias}

\subsection{Quatérnions}

Os quatérnions foram descobertos pelo matemático e físico irlandês William Rowan Hamilton. Eles são denotados pelo símbolo $\mathbb{H}$ em homenagem a Hamilton e são definidos da seguinte forma:

Definição 2.1. Os quatérnions são uma álgebra real $\mathbb{H}$ gerada pelos elementos $1, i, j, k$, isto é,

$$
\mathbb{H}=\{a+b i+c j+d k ; a, b, c, d \in \mathbb{R}\},
$$

onde a soma é definida por:

$$
\sum_{k=0}^{3} a_{k} i_{k}+\sum_{k=0}^{3} b_{k} i_{k}=\sum_{k=0}^{3}\left(a_{k}+b_{k}\right) i_{k}
$$

onde $i_{0}=1, i_{1}=i, i_{2}=j$ e $i_{3}=k$, e o produto satisfaz a lei distributiva e as relações: $i j=-j i=k, j k=$ $-k j=i, k i=-i k=j$ e $i^{2}=j^{2}=k^{2}=-1$.

Definição 2.2. Definimos o conjugado $\bar{q}$ de $q=a+b i+c j+k d$ por $\bar{q}=a-b i-c j-d k$ e definimos as parte real e imaginária de $q$ por $\operatorname{Re}(q)=a \in \mathbb{R}$ e $\operatorname{Im}(q)=b i+c j+d k$, respectivamente.

Podemos escrever cada quatérnion como um par de números complexos usando a equação

$$
a+i b+j c+k d=(a+b i)+(c+d i) j
$$

e dessa forma obtemos a expressão $q=\alpha+\beta j \in \mathbb{H} \cong \mathbb{C}^{2}$. Também podemos identificar os quatérnions com matrizes complexas $2 \times 2, M_{2}(\mathbb{C})$ por meio do isomorfismo $\imath: \mathbb{H} \rightarrow H \subset M_{2}(\mathbb{C})$ definido por

$$
\alpha+\beta j \longmapsto\left(\begin{array}{cc}
\alpha & \beta \\
-\bar{\beta} & \bar{\alpha}
\end{array}\right)
$$

A álgebra dos quatérnions pode assim ser realizada como uma subálgebra real de $M_{2}(\mathbb{C})$, usando as identificações

$$
1=\left(\begin{array}{ll}
1 & 0 \\
0 & 1
\end{array}\right), i=\left(\begin{array}{ll}
i & 0 \\
0 & -i
\end{array}\right), j=\left(\begin{array}{ll}
0 & 1 \\
-1 & 0
\end{array}\right), k=\left(\begin{array}{ll}
0 & i \\
i & 0
\end{array}\right) .
$$

Através do isomorfismo $\boldsymbol{l}$ podemos deduzir que $\mathbb{H}$ é uma álgebra associativa, não comutativa e com divisão, a inversa de qualquer matriz $A \in H$ está também em $H$ e a única matriz em $H$ cujo determinante é zero é a matriz nula.

Um importante grupo de matrizes de quatérnions que iremos precisar no decorrer do trabalho será definida a seguir:

Definição 2.3. O grupo simplético $S p(n)$ é o subgrupo de $G L(n, \mathbb{H})$ das matrizes com coeficientes em $\mathbb{H}$, 
inversíveis e que preservam o produto Hermitiano em $\mathbb{H}^{n}$ :

$$
\langle x, y\rangle=\sum_{k=i}^{n} \bar{x}_{k} y_{k}
$$

Isto significa que se $A \in S p(n)$ então $A^{*} A=A A^{*}=\mathrm{Id}$, ou seja, $S p(n)$ é o grupo das matrizes unitárias de $\mathbb{H}$, $U(n, \mathbb{H})$.

Dessa forma, o grupo simplético $S p(n)$ é o grupo de isometrias do espaço $\mathbb{H}^{n}$.

\subsection{Espaços Projetivos}

Seja $\mathbb{K}=\mathbb{C}$ ou $\mathbb{K}=\mathbb{H}$ e $a=\operatorname{dim}_{\mathbb{R}} \mathbb{K}$. Podemos dar a $\mathbb{K}^{n+1}$ uma estrutura de espaço vetorial à direita com produto por escalar definido por

$$
x \cdot \lambda=\left(x_{1}, \ldots, x_{n+1}\right) \cdot \lambda=\left(x_{1} \cdot \lambda, \ldots, x_{n+1} \cdot \lambda\right),
$$

produto Hermitiano

$$
\langle x, y\rangle=\sum_{i=1}^{n} \bar{x}_{i} \cdot y_{i}
$$

e produto escalar real

$$
\langle x, y\rangle_{\mathbb{R}}=\operatorname{Re}\langle x, y\rangle .
$$

Definição 2.4. O espaço projetivo $\mathbb{K} P^{n}$ é o espaço de órbitas para a ação à direita do grupo $\mathbb{K}^{*}=\mathbb{K}-\{0\}$ em $\mathbb{K}^{n+1}-\{0\}$, isto é, $x \sim y$ se, e somente se, existe um $\lambda \in \mathbb{K}^{*}$ tal que $x=y \cdot \lambda$. Denotaremos por $\pi(x)$ a órbita de $x$.

\subsection{1 $\mathbb{K} P^{n}$ como uma variedade}

O espaço projetivo $\mathbb{K} P^{n}$ é uma variedade diferenciável $C^{\infty}$ de dimensão $n a$, onde suas cartas são definidas da seguinte forma:

Seja

$$
U_{i}=\left\{x=\left(x^{1}, \ldots, x^{n+1}\right) \in \mathbb{K}^{n+1} ; x^{i} \neq 0\right\}, \quad 1 \leq i \leq n+1 .
$$

Como $U_{i}$ é aberto para todo $1 \leq i \leq n+1$, então os conjuntos $\pi\left(U_{i}\right)=V_{i}$ são abertos da topologia quociente em $\mathbb{K} P^{n}$. As funções $f_{i}: V_{i} \rightarrow \mathbb{K}^{n} \cong \mathbb{R}^{n a}$ definidas por

$$
f_{i}(\pi(x))=\left(\frac{x^{1}}{x^{i}}, \ldots, \frac{x^{i-1}}{x^{i}}, \frac{x^{i+1}}{x^{i}}, \ldots, \frac{x^{n+1}}{x^{i}}\right)
$$

são cartas para $\mathbb{K} P^{n}$ e suas inversas são

$$
f_{i}^{-1}\left(x^{1}, \ldots, \widehat{x^{i}}, \ldots, x^{n+1}\right)=\left(x^{1}, \ldots, 1, \ldots, x^{n+1}\right)
$$

onde $\widehat{x^{i}}$ significa que a i-ésima entrada está faltando.

Definição 2.5. Um fibrado principal sobre $\mathrm{M}$ com grupo estrutural $\mathrm{G}$ é uma variedade $\mathrm{P}$ tal que:

(1) G age livremente (à direita) em P: se para todo $x \in P$ tivermos $x \cdot g=x$ então $g=I$, isto é, somente o elemento identidade de $G$ fixa qualquer $x$.

(2) Existe uma projeção suave $\pi: P \rightarrow M$ e $M \cong P / G$

(3) P é localmente trivial: se $m \in M$, existe U vizinhança de $m$ tal que $\pi^{-1}(U) \cong U \times G$. 
O conjunto $\left(\mathbb{K}^{n+1}-\{0\}, \mathbb{K} P^{n}, \pi, \mathbb{K}^{*}\right)$ tem uma estrutura de um fibrado principal. As trivializações locais são dadas por

$$
\begin{aligned}
g_{i}: U_{i}=\pi^{-1}\left(V_{i}\right) & \rightarrow V_{i} \times \mathbb{K}^{n} \\
x & \mapsto(\pi(x), x)
\end{aligned}
$$

e as funções de transição por

$$
\begin{aligned}
V_{i} \cap V_{j} & \rightarrow \mathbb{K}^{*} \\
\pi(x) & \mapsto \frac{x^{i}}{x^{j}} .
\end{aligned}
$$

Notação 2.6. Denotaremos por $S \mathbb{K}^{n+1}$ (em vez de $S^{n a+a-1}$ ) a esfera unitária em $\mathbb{K}^{n+1}$ definida pela equação $\langle x, x\rangle=1$. A dimensão desse espaço é $n a+a-1$.

Assim, a esfera $S \mathbb{K}=S^{a-1}$ é um subgrupo de $\mathbb{K}^{*}$ tal que

$$
S \mathbb{K} \rightarrow S \mathbb{K}^{n+1} \rightarrow \mathbb{K} P^{n}
$$

é um subfibrado principal do anterior.

\subsubsection{Espaço tangente de $\mathbb{K} P^{n}$}

O fibrado tangente de $S \mathbb{K}^{n+1}$ é identificado classicamente da seguinte forma:

$$
T S \mathbb{K}^{n+1}=\left\{(x, u) ; x \in S \mathbb{K}^{n+1}, u \in \mathbb{K}^{n+1} e\langle x, u\rangle_{\mathbb{R}}=0\right\}
$$

e o espaço tangente de $\mathbb{K} P^{n}$ em $\pi(x)$ é isomorfo ao conjunto de classes

$$
\{(x \lambda, u \lambda) ;\langle x, u\rangle=0, \lambda \in S \mathbb{K}\} \cong T_{\pi(x)} \mathbb{K} P^{n} .
$$

Para mais detalhes veja ([Bes12], pág 73). Denotaremos por $\pi(x, u)$ o vetor tangente à $\mathbb{K} P^{n}$ em $\pi(x)$.

\subsubsection{Métrica Riemanniana Canônica em $\mathbb{K} P^{n}$}

Veja que para $u, v \in \mathbb{K}^{n+1}$ e $\lambda \in S \mathbb{K}$ temos

$$
\begin{aligned}
2\langle u \lambda, v \lambda\rangle_{\mathbb{R}} & =\langle u \lambda, v \lambda\rangle+\overline{\langle u \lambda, v \lambda\rangle} \\
& =\langle u \lambda, v \lambda\rangle+\langle v \lambda, u \lambda\rangle \\
& =\bar{\lambda}\langle u, v\rangle \lambda+\bar{\lambda}\langle v, u\rangle \lambda \\
& =\bar{\lambda}(\langle u, v\rangle+\overline{\langle u, v\rangle}) \lambda \\
& =\bar{\lambda} 2\langle u, v\rangle_{\mathbb{R}} \lambda \\
& =2\langle u, v\rangle_{\mathbb{R}}
\end{aligned}
$$

Logo,

$$
\langle u, v\rangle_{\mathbb{R}}=\langle u \lambda, v \lambda\rangle_{\mathbb{R}}
$$

para todo $u, v \in \mathbb{K}^{n+1}$ e $\lambda \in S \mathbb{K}$. Assim, podemos dotar o espaço projetivo $\mathbb{K} P^{n}$ com uma métrica Riemanniana $g$ definida por

$$
g(\pi(x, u), \pi(x, v)):=\langle u, v\rangle_{\mathbb{R}} .
$$

Logo, temos $g$ como uma métrica natural em $\mathbb{K} P^{n}$.

Note ainda que para $\mathbb{K}=\mathbb{C},\langle u, v\rangle$ é invariante pela ação de $S^{1}$ mas para $\mathbb{K}=\mathbb{H},\langle u, v\rangle$ não é invariante sob $S^{3}$ porque $\mathbb{H}$ não é comutativo. 


\subsubsection{Grupo de Isometrias em $\mathbb{K} P^{n}$}

Seja $U(n+1, \mathbb{K})$ o subgrupo do grupo linear sobre $\mathbb{K}, G L(n+1, \mathbb{K})$, que deixa o produto Hermitiano $\langle$,$\rangle invariante, isto é,$

$$
\forall A \in U(n+1, \mathbb{K}) \Longrightarrow\langle A(x), A(y)\rangle=\langle x, y\rangle .
$$

Então,

$$
\begin{aligned}
& U(n+1, \mathbb{C})=U(n+1), \text { o grupo unitário } \\
& U(n+1, \mathbb{H})=S p(n+1), \text { o grupo simplético }
\end{aligned}
$$

Definição 2.7. O grupo projetivo $P U(n+1, \mathbb{K})$ é a ação do grupo $U(n+1, \mathbb{K})$ no espaço projetivo $\mathbb{K} P^{n}$, ou seja, $P U(n+1, \mathbb{K})$ é o grupo quociente

$$
P U(n+1, \mathbb{K})=U(n+1, \mathbb{K}) / Z(U(n+1, \mathbb{K}))
$$

onde $Z(U(n+1, \mathbb{K}))$ é o subgrupo das matrizes unitárias escalares $\{\operatorname{Id} \cdot \lambda ;\langle\lambda, \lambda\rangle=1\}$.

O grupo $Z(U(n+1, \mathbb{K}))$ age trivialmente em $\mathbb{K} P^{n}$ e a notação $Z$ deve-se ao fato de que o subgrupo das matrizes escalares é o centro de $U(n+1, \mathbb{K})$.

$\operatorname{Dados} \pi(x), \pi(y) \in \mathbb{K} P^{n}$ temos para todo $A \cdot I \lambda \in U(n+1, \mathbb{K}) / Z(U(n+1, \mathbb{K}))$

$$
\begin{aligned}
\langle A \cdot \operatorname{Id} \cdot \lambda(\pi(x)), A \cdot \operatorname{Id} \cdot \lambda(\pi(y))\rangle & =\langle A(\pi(x)) \cdot \lambda, A(\pi(y)) \cdot \lambda\rangle \\
& =\langle A(\pi(x) \cdot \lambda), A(\pi(y) \cdot \lambda)\rangle \\
& =\langle A(\pi(x)), A(\pi(y))\rangle \\
& =\langle\pi(x), \pi(y)\rangle
\end{aligned}
$$

Logo, $P U(n+1, \mathbb{K})$ define as isometrias de $\mathbb{K} P^{n}$ onde

$$
\begin{gathered}
P U(n+1, \mathbb{C})=U(n+1) / Z(U(n+1)), \text { o grupo projetivo unitário } \\
P U(n+1, \mathbb{H})=S p(n+1) / Z(U(n+1)), \text { o grupo projetivo simplético. }
\end{gathered}
$$

\subsubsection{Geodésicas em $\mathbb{K} P^{n}$}

Proposição 2.8 ([Bes12], pág 81). Todas as geodésicas dos espaços projetivos $\mathbb{K} P^{n}$ são fechadas, simples e tem comprimento $\pi$. então:

Seja $\gamma$ uma geodésica em $\mathbb{K} P^{n}$ com condições iniciais $\gamma(0)=p=\pi(x), \dot{\gamma}(0)=X=\pi(x, u)$ e $\langle X, X\rangle=1$,

Proposição 2.9 ([Bes12], pág 81-82). A geodésica $\gamma$ em $\mathbb{K} P^{n}$ tem equação

$$
\gamma(s)=\pi(x \cos s+u \sin s)=\exp _{p} s X .
$$




\section{Capítulo 3}

\section{Cohomologia de De Rham}

\subsection{Cadeias e Cocadeias}

Nessa seção trataremos de módulos sob um anel geral $\mathrm{R}($ ou $\mathbb{Z}$ ) e não especificamente sob variedades.

Definição 3.1. Sejam $M$ e $N$ módulos sob R. Uma função $f: M \rightarrow N$ diz-se um homomorfismo se para todos $m, m_{1}, m_{2} \in M$ e $r \in R$ tem-se

(i) $f\left(m_{1}+m_{2}\right)=f\left(m_{1}\right)+f\left(m_{2}\right)$

(ii) $f(r m)=r f(m)$

Definição 3.2. Uma sequência $C_{\bullet}=\left(C_{n}, \partial_{n} \mid n \in \mathbb{Z}\right)$ de módulos $C_{n}$ e homomorfismos $\partial_{n}: C_{n} \rightarrow C_{n-1}$ é chamado de um complexo de cadeia se para todo $n \in \mathbb{Z}$ tivermos $\partial_{n-1} \circ \partial_{n}=0$.

Um complexo de cadeia é usualmente visualizada em um diagrama tal como

$$
\cdots \stackrel{\partial_{n+1}}{\longrightarrow} C_{n} \stackrel{\partial_{n}}{\longrightarrow} C_{n-1} \stackrel{\partial_{n-1}}{\longrightarrow} \cdots
$$

E podemos observar que $\partial_{n-1} \circ \partial_{n}=0$ se, e somente se, $\operatorname{Im}\left(\partial_{n}\right) \subset \operatorname{ker}\left(\partial_{n-1}\right)$.

Definição 3.3. Chamamos a sequência (3.1) de exata se, e somente se, $\operatorname{Im}\left(\partial_{n}\right)=\operatorname{ker}\left(\partial_{n-1}\right)$ para todo $n \in \mathbb{Z}$. Definição 3.4. Uma sequência $C^{\bullet}=\left(C^{n}, d_{n} \mid n \in \mathbb{Z}\right)$ de módulos $C^{n}$ e homomorfismos $d_{n}: C^{n} \rightarrow C^{n+1}$ é chamada de um complexo de cocadeia se para todo $n \in \mathbb{Z}$ tivermos $d_{n+1} \circ d_{n}=0$.

$$
\stackrel{d_{n-1}}{\longrightarrow} C^{n} \stackrel{d_{n}}{\longrightarrow} C^{n+1} \stackrel{d_{n+1}}{\longrightarrow} \cdots
$$

Sejam $M, M^{\prime}$ e $N$ módulos sob um anel $\mathrm{R}$ ( ou $\mathbb{Z}$ ), então:

Observação 3.5. A sequência $0 \longrightarrow M^{\prime} \stackrel{f}{\longrightarrow} M$ é exata se, e somente se, $f$ é injetora.

Observação 3.6. A sequência $M \stackrel{f}{\longrightarrow} M^{\prime} \longrightarrow 0$ é exata se, e somente se, $f$ é sobrejetora.

Observação 3.7. Uma sequência $0 \longrightarrow M \stackrel{f}{\longrightarrow} N \longrightarrow 0$ é exata se, e somente se, $f$ é um isomorfismo.

Definição 3.8. Uma sequência exata da forma

$$
0 \longrightarrow M^{\prime} \stackrel{f}{\longrightarrow} M \stackrel{g}{\longrightarrow} M^{\prime \prime} \longrightarrow 0
$$

chama-se uma sequência exata curta.

Definição 3.9. Diz-se que uma sequência exata curta

$$
0 \longrightarrow M^{\prime} \stackrel{f}{\longrightarrow} M \stackrel{g}{\longrightarrow} M^{\prime \prime} \longrightarrow 0
$$

cinde se $N=\operatorname{Im}(f)=\operatorname{ker}(g)$ é um somando direto de M. 
Lema 3.10 (Lema de Splitting). Dada uma sequência exata curta de R-módulos

$$
0 \longrightarrow M^{\prime} \stackrel{f}{\longrightarrow} M \stackrel{g}{\longrightarrow} M^{\prime \prime} \longrightarrow 0
$$

as seguintes afirmações são equivalentes:

(i) A sequência (3.2) cinde.

(ii) Existe um homomorfismo $\psi: M \rightarrow M^{\prime}$ tal que $\psi \circ f=I d_{M^{\prime}}$.

(iii) Existe um homomorfismo $\varphi: M^{\prime \prime} \rightarrow M$ tal que $g \circ \varphi=I d_{M^{\prime \prime}}$.

Nessas condições $M \cong M^{\prime} \oplus M^{\prime \prime}$.

\subsection{Complexos de De Rham}

Sejam M uma variedade diferenciável de dimensão n e $x^{1}, \ldots, x^{n}$ suas coordenadas locais.

Definição 3.11. Definimos $\Omega^{*}$ como a álgebra sobre $\mathbb{R}$ gerada por $d x^{1}, \ldots, d x^{n}$ com as relações

$$
\left\{\begin{array}{l}
d x^{i} \wedge d x^{i}=0 \\
d x^{i} \wedge d x^{j}=-d x^{j} \wedge d x_{i}, i \neq j
\end{array}\right.
$$

Também podemos ver $\Omega^{*}$ como o espaço vetorial real cuja base é

$$
1, d x^{i}, d x^{i} \wedge d x^{j}, d x^{i} \wedge d x^{j} \wedge d x^{k}, \ldots, d x^{1} \wedge \cdots \wedge d x^{n}
$$

onde $i<j<k$. As formas diferenciais $C^{\infty}$ em M são elementos de

$$
\Omega^{*}(M)=\left\{\text { funções } C^{\infty} \text { em } \mathrm{M}\right\} \otimes_{\mathbb{R}} \Omega^{*} .
$$

Assim, podemos definir uma k-forma $\alpha$ em $\Omega^{*}(M)$ como

$$
\alpha=\sum f_{i_{1} \ldots i_{k}} d x^{i_{1}} \wedge \cdots \wedge d x^{i_{k}}
$$

onde os coeficientes $f_{i_{1} \ldots i_{k}}$ são funções $C^{\infty}$, ou de outra forma

$$
\alpha=\sum_{I} f_{I} d x_{I}
$$

Se $\Omega^{k}(M)$ denota o conjunto das k-formas em $M$ temos

$$
\Omega^{*}(M)=\bigoplus_{k=1}^{n} \Omega^{k}(M)
$$

Observação 3.12. $\Omega^{k}(M)=0$ para $k>\operatorname{dim}(M)$ e $k<0$.

Definição 3.13. Definimos o operador diferencial $d: \Omega^{k}(M) \rightarrow \Omega^{k+1}(M)$ pelas seguintes regras:

(i) se $f \in \Omega^{0}(M)$ então $d f=\sum \frac{\partial f}{\partial x_{i}} d x_{i}$

(ii) se $\alpha=\sum f_{I} d x_{I}$ então $d \alpha=\sum d f_{I} \wedge d x_{I}$

Chamamos $d$ de derivada exterior.

Proposição 3.14. Se $\alpha$ é uma k-forma e $\beta$ uma $r$-forma temos

$$
d(\alpha \wedge \beta)=d \alpha \wedge \beta+(-1)^{k} \alpha \wedge d \beta .
$$


Demonstração. Como $d$ é linear, basta verificar o caso que $\alpha=f_{I} d x_{I}$ e $\beta=g_{J} d x_{j}$.

$$
\begin{aligned}
d(\alpha \wedge \beta) & =d\left(f_{I} g_{J}\right) d x_{I} \wedge d x_{J} \\
& =\left[\left(d f_{I}\right) g_{J}+f_{I}\left(d g_{J}\right)\right] \wedge d x_{I} \wedge d x_{J} \\
& =g_{J} d f_{I} \wedge d x_{I} \wedge d x_{J}+f_{I} d g_{J} \wedge d x_{I} \wedge d x_{J} \\
& =g_{J} d f_{I} \wedge d x_{I} \wedge d x_{J}+(-1)^{k} f_{I} d x_{I} \wedge d g_{J} \wedge d x_{J} \\
& =(d \alpha) \wedge \beta+(-1)^{k} \alpha \wedge d \beta .
\end{aligned}
$$

Proposição 3.15. $d^{2}=0$.

Demonstração. Para $f \in \Omega^{0}(M)$, isto é, f uma função em $\mathrm{M}$ temos

$$
d^{2} f=d\left(\sum_{i} \frac{\partial f}{\partial x^{i}} d x^{i}\right)=\sum_{i, j} \frac{\partial^{2} f}{\partial x^{j} \partial x^{i}} d x^{j} \wedge d x^{i}
$$

Como os fatores $\frac{\partial^{2} f}{\partial x^{j} \partial x^{i}}$ são simétricos e $d x^{j} \wedge d x^{i}$ são antissimétricos temos

$$
\frac{\partial^{2} f}{\partial x^{j} \partial x^{i}} d x^{j} \wedge d x^{i}+\frac{\partial^{2} f}{\partial x^{i} \partial x^{j}} d x^{i} \wedge d x^{j}=\frac{\partial^{2} f}{\partial x^{j} \partial x^{i}} d x^{j} \wedge d x^{i}-\frac{\partial^{2} f}{\partial x^{j} \partial x^{i}} d x^{j} \wedge d x^{i}=0
$$

$\mathrm{e}$

$$
\frac{\partial^{2} f}{\partial x^{i} \partial x^{i}} d x_{i} \wedge d x^{i}=0
$$

Logo,

$$
d^{2} f=\sum_{i, j} \frac{\partial^{2} f}{\partial x^{j} \partial x^{i}} d x^{j} \wedge d x^{i}=0 .
$$

Para formas, como $d$ é um operador linear, é suficiente mostrar o caso quando $\alpha=f_{I} d x_{I}$ é uma $k$-forma. Pela Proposição 3.14 e a primeira parte da demonstração segue que

$$
d^{2} \alpha=d^{2}\left(f_{I} d x_{I}\right)=d\left(d f_{I} \wedge d x_{I}\right)=d^{2} f_{I} \wedge d x_{I}+(-1)^{k} d f_{I} \wedge d^{2} x_{I}=0 .
$$

Definição 3.16. Uma k-forma $\alpha$ é dita fechada se $d \alpha=0$ e é dita exata se existe uma $(k-1)$-forma $\beta$ tal que $d \beta=\alpha$.

Definição 3.17. O complexo $\Omega^{*}(M)$ com o operador diferencial d é chamado de complexo de De Rham em M.

Observação 3.18. O núcleo de $d: \Omega^{k}(M) \rightarrow \Omega^{k+1}(M)$ são formas fechadas e a imagem de d são formas exatas. Pela Proposição 3.15 segue que toda forma exata é fechada.

Considere o seguinte diagrama

$$
\cdots \longrightarrow \Omega^{k-1}(M) \stackrel{d_{k-1}}{\longrightarrow} \Omega^{k}(M) \stackrel{d_{k}}{\longrightarrow} \Omega^{k+1} \longrightarrow \cdots
$$

onde $d_{i}, i \geq 1$ é a derivada exterior. Pela Proposição 3.15 segue que $d_{k} \circ d_{k-1}=0$. Logo, $\operatorname{Im}\left(d_{k-1}\right) \subseteq k e r\left(d_{k}\right)$. Assim, podemos definir

Definição 3.19. A k-ésima cohomologia de De Rham de M é o espaço vetorial

$$
H_{D R}^{k}(M)=\frac{k e r\left(d_{k}\right)}{\operatorname{Im}\left(d_{k-1}\right)}=\frac{\{\mathrm{k} \text {-formas fechadas }\}}{\{\mathrm{k} \text {-formas exatas }\}} .
$$


As vezes escreveremos $H^{k}(M)$ em vez de $H_{D R}^{k}(M)$ e denotaremos a classe de cohomologia de uma forma $\alpha$ por $[\alpha]$.

Definição 3.20. A dimensão do k-ésimo grupo de cohomologia de uma variedade M é chamado de número de Betti e o denotamos por

$$
b_{k}(M)=\operatorname{dim} H^{k}(M) \text {. }
$$

\subsection{Lema de Poincaré}

Sejam $x^{1}, \ldots, x^{m}$ e $y^{1}, \ldots, y^{n}$ coordenadas locais de $\mathrm{M}$ e $\mathrm{N}$, respectivamente. Uma aplicação suave $f$ : $M \rightarrow N$ induz um pullback de funções $C^{\infty}, f^{*}: \Omega^{0}(N) \rightarrow \Omega^{0}(M)$ definido por:

$$
f^{*}(g)=g \circ f .
$$

Podemos estender esse pullback para todas as formas, $f^{*}: \Omega^{*}(N) \rightarrow \Omega^{*}(M)$ de tal forma que $f^{*}$ comute com d da seguinte maneira:

$$
f^{*}\left(\sum g_{I} d y^{i_{1}} \wedge \cdots \wedge d y^{i_{k}}\right)=\sum\left(g_{I} \circ f\right) d f_{i_{1}} \wedge \cdots \wedge d f_{i_{k}}
$$

onde $f_{i}=y^{i} \circ f$ é a i-ésima componente da função $\mathrm{f}$.

Proposição 3.21. $f^{*}$ como definido acima comuta com $d$.

Demonstração. Como

$$
\begin{aligned}
d f^{*}\left(g_{I} d y^{i_{1}} \wedge \cdots \wedge d y^{i_{k}}\right) & =d\left[\left(g_{I} \circ f\right) d f_{i_{1}} \wedge \cdots \wedge d f_{i_{k}}\right] \\
& =d\left(g_{I} \circ f\right) \wedge d f_{i_{1}} \wedge \cdots \wedge d f_{i_{k}} .
\end{aligned}
$$

e

$$
\begin{aligned}
f^{*} d\left(g_{I} d y^{i_{1}} \wedge \cdots \wedge d y^{i_{k}}\right) & =f^{*}\left[\sum_{i=1}^{n} \frac{\partial g_{I}}{\partial y^{i}} d y^{i} \wedge d y^{i_{1}} \wedge \cdots \wedge d y^{i_{k}}\right] \\
& =\sum_{i=1}^{n}\left[\left(\frac{\partial g_{I}}{\partial y^{i}} \circ f\right) d f_{i}\right] d y^{i_{1}} \wedge \cdots \wedge d y^{i_{k}} \\
& =d\left(g_{I} \circ f\right) d y^{i_{1}} \wedge \cdots \wedge d y^{i_{k}}
\end{aligned}
$$

temos $d f^{*}=f^{*} d$.

Lema 3.22. Sejam $M$ e $N$ variedades e $f: M \rightarrow N$ uma função suave então o pullback manda formas fechadas em $N$ em formas fechadas em $M$ e formas exatas em $N$ em formas exatas em $M$. Assim, $f^{*}$ : $H_{D R}^{k}(N) \rightarrow H_{D R}^{k}(M)$.

Demonstração. Seja $\alpha$ uma forma fechada em N então $d \alpha=0$. Pela Proposição 3.21 temos

$$
0=f^{*} d \alpha=d f^{*} \alpha .
$$

Logo, $f^{*} \alpha$ é fechada. Agora seja $\beta$ uma forma exata em $\mathrm{N}$, então, existe $\eta$ tal que $\beta=d \eta$. Pela Proposição 3.21 temos

$$
f^{*} \beta=f^{*} d \eta=d f^{*} \eta
$$

ou seja, $f^{*} \beta$ é exata.

Sejam $\pi: \mathbb{R}^{n} \times \mathbb{R} \rightarrow \mathbb{R}$ a projeção no primeiro fator e $s: R^{n} \rightarrow \mathbb{R}^{n} \times \mathbb{R}$ a seção nula, isto é, a função que aplica $x \mapsto(x, 0)$.

Proposição 3.23. As aplicações $\pi^{*}: H^{*}\left(\mathbb{R}^{n}\right) \rightarrow H^{*}\left(\mathbb{R}^{n} \times \mathbb{R}\right)$ e $s^{*}: H^{*}\left(\mathbb{R}^{n} \times \mathbb{R}\right) \rightarrow H^{*}\left(\mathbb{R}^{n}\right)$ são isomorfismos. 
Demonstração. Como $\pi \circ s=I d$ então:

- Se $f \in \Omega^{0}\left(\mathbb{R}^{n}\right)$ temos

$$
\left(s^{*} \circ \pi^{*}\right) f=s^{*}(f \circ \pi)=f \circ \pi \circ s=f
$$

- Se $\alpha=f d x^{1} \wedge \cdots \wedge d x^{k} \in \Omega^{k}\left(\mathbb{R}^{n}\right), k \geq 1$, temos

$$
\begin{aligned}
\left(s^{*} \circ \pi^{*}\right)(\alpha) & =s^{*}\left(f \circ \pi d x^{1} \wedge \cdots \wedge d x^{k}\right) \\
& =(f \circ \pi \circ s) d x^{1} \wedge \cdots \wedge d x^{k} \\
& =f d x^{1} \wedge \cdots \wedge d x^{k} \\
& =\alpha
\end{aligned}
$$

Logo, $s^{*} \circ \pi^{*}=I d$. No entanto, como $s \circ \pi \neq I d$ temos $\pi^{*} \circ s^{*} \neq I d$ no nível de formas pois, por exemplo, se $f \in \Omega^{0}\left(\mathbb{R}^{n} \times \mathbb{R}\right)$ temos

$$
\pi^{*} \circ s^{*}(f(x, t))=f \circ s \circ \pi(x, t)=f \circ s(x)=f(x, 0) .
$$

Para mostrar que $\pi^{*} \circ s^{*}$ é a identidade na cohomologia é suficiente encontrar uma aplicação $K$ definida em $\Omega^{*}\left(\mathbb{R}^{n} \times \mathbb{R}\right)$ tal que

$$
I d-\pi^{*} \circ s^{*}= \pm(d K-K d)
$$

onde $d K \pm K d$ aplica formas fechadas em formas exatas, dessa forma, aplicando $[\alpha] \in H^{*}\left(\mathbb{R}^{n} \times \mathbb{R}\right)$ na classe nula.

Observe que toda forma em $\mathbb{R}^{n} \times \mathbb{R}$ é uma combinação linear de dois tipos de formas:

(I) $\left(\pi^{*} \alpha\right) f(x, t)$

(II) $\left(\pi^{*} \alpha\right) \wedge f(x, t) d t$

onde $\alpha$ é uma forma em $\mathbb{R}^{n}$. Definimos $K: \Omega^{k}\left(\mathbb{R}^{n} \times \mathbb{R}\right) \rightarrow \Omega^{k-1}\left(\mathbb{R}^{n} \times \mathbb{R}\right)$ por:

(I) $\left(\pi^{*} \alpha\right) f(x, t) \longmapsto 0$

(II) $\left(\pi^{*} \alpha\right) \wedge f(x, t) d t \longmapsto\left(\pi^{*} \alpha\right) \int_{0}^{t} f(x, t) d t$

Agora vamos verificar se a aplicação $K$ como definida acima satisfaz a equação (3.3). Para formas do tipo I, $\beta=\left(\pi^{*} \alpha\right) f(x, t)$, onde $\alpha \in \Omega^{k}\left(\mathbb{R}^{n}\right)$ temos

$$
\begin{aligned}
\left(I d-\pi^{*} \circ s^{*}\right) \beta & =\left(\pi^{*} \alpha\right) f(x, t)-\pi^{*} \circ s^{*}\left(\left(\pi^{*} \alpha\right) f(x, t)\right) \\
& =\left(\pi^{*} \alpha\right) f(x, t)-\pi^{*} \alpha f(x, 0)
\end{aligned}
$$

e,

$$
\begin{aligned}
(d K-K d) \beta & =d K(\beta)-K d \beta=-K d \beta \\
& =-K\left[\left(\pi^{*} d \alpha\right) f(x, t)+(-1)^{k}\left(\pi^{*} \alpha\right) \wedge\left(\sum_{i} \frac{\partial f}{\partial x^{i}} d x^{i}+\frac{\partial f}{\partial t} d t\right)\right] \\
& =-K\left(\left(\pi^{*} d \alpha\right) f(x, t)\right)+(-1)^{k+1} K\left(\left(\pi^{*} \alpha\right) \wedge \sum_{i} \frac{\partial f}{\partial x^{i}} d x^{i}\right)+(-1)^{k+1} K\left(\left(\pi^{*} \alpha\right) \wedge \frac{\partial f}{\partial t} d t\right) \\
& =(-1)^{k+1}\left(\pi^{*} \alpha\right) \int_{0}^{t} \frac{\partial f}{\partial t} d t \\
& =(-1)^{k+1}\left(\pi^{*} \alpha\right)[f(x, t)-f(x, 0)]
\end{aligned}
$$

Logo, $\left(I d-\pi^{*} \circ s^{*}\right) \beta=(-1)^{k+1}(d K-K d) \beta$. 
Para formas do tipo II, $\beta=\left(\pi^{*} \alpha\right) \wedge f(x, t) d t \in \Omega^{k}\left(\mathbb{R}^{n} \times \mathbb{R}\right)$ temos

$$
\left(I d-\pi^{*} \circ s^{*}\right) \beta=\beta-\pi^{*} \circ s^{*}\left(\left(\pi^{*} \alpha\right) \wedge f(x, t) d t\right)=\beta
$$

pois $s^{*}(d t)=d\left(s^{*} t\right)=d(0)=0$. Por outro lado,

$$
\begin{aligned}
K d \beta & =K\left[\pi^{*}(d \alpha) \wedge f(x, t) d t+(-1)^{k-1} \pi^{*} \alpha \wedge\left(\sum_{i} \frac{\partial f}{\partial x^{i}} d x^{i} \wedge d t+\frac{\partial f}{\partial t} d t \wedge d t\right)\right] \\
& =K\left[\pi^{*}(d \alpha) \wedge f(x, t) d t+(-1)^{k-1} \pi^{*} \alpha \wedge\left(\sum_{i} \frac{\partial f}{\partial x^{i}} d x^{i} \wedge d t\right)\right] \\
& =K\left[\pi^{*}(d \alpha) \wedge f(x, t) d t+(-1)^{k-1} \sum_{i} \pi^{*} \alpha \wedge d x^{i} \wedge\left(\frac{\partial f}{\partial x^{i}} d t\right)\right] \\
& =\pi^{*}(d \alpha) \int_{0}^{t} f(x, t) d t+(-1)^{k-1} \sum_{i} \pi^{*} \alpha \wedge d x^{i} \int_{0}^{t} \frac{\partial f}{\partial x^{i}} d t
\end{aligned}
$$

e,

$$
\begin{aligned}
d K \beta & =d\left[\pi^{*}(\alpha) \int_{0}^{t} f(x, t) d t\right] \\
& =\pi^{*}(d \alpha) \int_{0}^{t} f(x, t) d t+(-1)^{k-1}\left(\pi^{*} \alpha\right)\left[\sum_{i}\left(\int_{0}^{t} \frac{\partial f}{\partial x^{i}} d t\right) d x^{i}+f(x, t) d t\right] \\
& =\pi^{*}(d \alpha) \int_{0}^{t} f(x, t) d t+(-1)^{k-1} \sum_{i}\left(\pi^{*} \alpha\right) \wedge d x^{i} \int_{0}^{t} \frac{\partial f}{\partial x^{i}} d t+(-1)^{k-1}\left(\pi^{*} \alpha\right) f(x, t) d t
\end{aligned}
$$

Logo,

$$
(d K-K d) \beta=(-1)^{k-1}\left(\pi^{*} \alpha\right) \wedge f(x, t) d t=(-1)^{k-1} \beta=(-1)^{k-1}\left(I d-\pi^{*} \circ s^{*}\right) .
$$

Portanto, $H^{*}\left(\mathbb{R}^{n} \times \mathbb{R}\right)$ e isomorfo a $H^{*}\left(\mathbb{R}^{n}\right)$.

Corolário 3.24 (Lema de Poincaré).

$$
H^{k}\left(\mathbb{R}^{n}\right)=H^{k}(\text { ponto })= \begin{cases}\mathbb{R}, & \text { se } k=0 \\ 0, & \text { caso contrário. }\end{cases}
$$

Demonstração. Faça indução em n na Proposição 3.23.

O Corolário acima nos diz que toda forma fechada no $\mathbb{R}^{n}$ é exata. Como toda variedade diferenciável M é localmente homeomorfa a um aberto do $\mathbb{R}^{n}$ seque que toda forma fechada em M é localmente exata.

\subsection{Cohomologia de De Rham com suporte compacto e Cohomologia com- pacta vertical}

Definição 3.25. O suporte de uma função contínua em um espaço topológico é o fecho do conjunto onde $f$ é diferente de zero, isto é,

$$
\operatorname{Supp} f:=\overline{\{p \in X ; f(p) \neq 0\}} .
$$

Se na definição do complexo de De Rham usarmos somente funções $C^{\infty}$ com suporte compacto, então, o complexo resultante é chamado de complexo de De Rham com suporte compacto.

$$
\Omega_{c}^{*}(M)=\left\{\text { funções } C^{\infty} \text { em } M \text { com suporte compacto }\right\} \otimes_{\mathbb{R}} \Omega^{*} .
$$

A cohomologia desse complexo é denotada por $H_{c}^{*}(M)$. 
Definição 3.26. Seja $\pi: E \rightarrow M$ uma aplicação sobrejetora entre variedades tal que $\pi^{-1}(x)$ é um espaço vetorial para todo $x \in M$. A aplicação $\pi$ é chamada de um fibrado vetorial real de posto n quando existe uma cobertura aberta $\left\{U_{i}\right\}$ de $\mathrm{M}$ e difeomorfismos que preservam as fibras

$$
\phi_{i}:\left.E\right|_{U_{i}}=\pi^{-1}\left(U_{i}\right) \rightarrow U_{i} \times \mathbb{R}^{n}
$$

que são isomorfismos lineares em cada fibra.

Definição 3.27. Os complexos de formas com suporte compacto na direção vertical, $\Omega_{c v}^{k}(E)$, são definidos da seguinte forma: uma k-forma $\alpha$ em E está em $\Omega_{c v}^{k}(E)$ se, e somente se, para cada conjunto compacto $K \subset M, \pi^{-1}(K) \cap \operatorname{Supp} \alpha$ é compacto.

Definição 3.28. A cohomologia dos complexos $\Omega_{c v}^{k}(E)$ denotados por $H_{c v}^{*}(E)$, é chamada a cohomologia de E com suporte compacto na direção vertical, ou cohomologia compacta vertical.

Seja E um fibrado vetorial. Sejam $x^{1}, \ldots, x^{m}$ as coordenadas locais de $\mathrm{Me} t_{1}, \ldots, t_{n}$ as coordenadas locais de E. Então, as formas de E são de dois tipos:

(I) $\left(\pi^{*} \alpha\right) \wedge f\left(x, t_{1}, \ldots, t_{n}\right) d t_{i_{1}} \wedge \cdots \wedge d t_{i_{r}}, \quad r<n$

(II) $\left(\pi^{*} \alpha\right) \wedge f\left(x, t_{1}, \ldots, t_{n}\right) d t_{1} \wedge \cdots \wedge d t_{n}$.

Definição 3.29. Definimos a integração ao longo das fibras $\pi_{*}: \Omega_{c v}^{*}(E) \rightarrow \Omega^{*-n}(M)$ por

(I) $\left(\pi^{*} \alpha\right) \wedge f\left(x, t_{1}, \ldots, t_{n}\right) d t_{i_{1}} \wedge \cdots \wedge d t_{i_{r}} \longmapsto 0, \quad r<n$

(II) $\left(\pi^{*} \alpha\right) \wedge f\left(x, t_{1}, \ldots, t_{n}\right) d t_{1} \wedge \cdots \wedge d t_{n} \longmapsto \alpha \int_{E} f\left(x, t_{1}, \ldots, t_{n}\right) d t_{1} \wedge \cdots \wedge d t_{n}$.

Proposição 3.30. A integração ao longo da fibra $\pi_{*}$ comuta com a derivada exterior $d$.

\subsection{Classe de Euler}

Nessa seção mostraremos a definição da classe de Euler de um fibrado vetorial $\pi: E \rightarrow M$ quando o posto de E é 2. O caso geral é similar porém mais complexo. A construção no caso geral pode ser encontrada em ([BT24], §11)

Definição 3.31. Seja $M$ uma variedade com atlas $\left\{\left(U_{i}, \phi_{i}\right)\right\}$. Dizemos que o atlas é orientado se todas as funções de transição $\phi_{i} \circ \phi_{j}^{-1}$ são difeomorfismos que preservam a orientação, isto é, o determinante do Jacobiano de $\phi_{i} \circ \phi_{j}^{-1}$ é positivo. A variedade é dita orientável se possui um atlas orientado.

Proposição 3.32. Uma variedade $M$ de dimensão $n$ é orientável se, e somente se, tem uma $n$-forma global que nunca se anula ([BT24], pág 29).

Seja $\mathrm{E}$ um fibrado vetorial orientado de posto n sobre $\mathrm{M}$ e seja $E^{0}=E \backslash\{0\}$, onde $\{0\}$ é a seção nula. Podemos dotar E com uma estrutura Riemanniana como segue. Seja $\left\{U_{i}\right\}$ uma cobertura aberta de M que trivializa E. Em cada $U_{i}$ escolha um frame ortogonal para $\left.E\right|_{U_{i}}$. Denote por $\langle,\rangle_{i}$ o produto interno em $\left.E\right|_{U_{i}}$ induzido pelo produto interno usual em $\mathbb{R}^{n}$. Agora use a partição da unidade $\left\{\rho_{i}\right\}$ para juntá-los, isto é, formar

$$
\langle,\rangle=\sum \rho_{i}\langle,\rangle_{i}
$$

Assim, podemos definir uma função raio em E. Suponha que E tem posto 2 e $\left\{U_{i}\right\}$ é uma cobertura aberta coordenada de $\mathrm{M}$ que trivializa E. Como E tem uma estrutura Riemanniana sobre cada $U_{i}$ podemos escolher um frame ortonormal. Isso define em $\left.E^{0}\right|_{U_{i}}$ coordenadas polares $r_{i}$ e $\theta_{i}$. Se $x_{1}, \ldots, x_{n}$ são coordenadas de $U_{i}$ então $\pi^{*} x_{1}, \ldots, \pi^{*} x_{n}, r_{i}, \theta_{i}$ são coordenadas em $E^{0} \mid U_{i}$.

$\mathrm{Na}$ interseção $U_{i} \cap U_{j}$ os raios $r_{i}$ e $r_{j}$ são iguais, mas as coordenadas angulares $\theta_{i}$ e $\theta_{j}$ diferem por uma rotação. Isso nos permite definir de forma não ambígua $\varphi_{i j}$ (a menos de múltiplos de $2 \pi$ ) como o ângulo de rotação na direção anti horária do sistema de coordenadas $i$ para o sistema de coordenadas $j$ :

$$
\theta_{j}=\theta_{i}+\pi^{*} \varphi_{i j}, \quad \varphi_{i j}: U_{i} \cap U_{j} \rightarrow \mathbb{R}
$$


Apesar da rotação de $i$ para $j$ e depois de $j$ para $k$ ser o mesmo que rotacionar de $i$ para $k$, no entanto, não é verdade que $\varphi_{i j}+\varphi_{j k}-\varphi_{i k}=0$. Apenas podemos dizer que

$$
\varphi_{i j}+\varphi_{j k}-\varphi_{i k} \in 2 \pi \mathbb{Z}
$$

Lema 3.33. Existem 1-formas $\xi_{i}$ em $U_{i}$ tais que

$$
\frac{1}{2 \pi} d \varphi_{i j}=\xi_{j}-\xi_{i}
$$

Demonstração. Defina $\xi_{i}=\frac{1}{2 \pi} \sum_{k} \rho_{k} d \varphi_{k i}$ onde $\left\{\rho_{k}\right\}$ é a partição da unidade subordinada a cobertura aberta $\left\{U_{k}\right\}$. Então,

$$
\xi_{j}-\xi_{i}=\frac{1}{2 \pi} \sum_{k} \rho_{k} d\left(\varphi_{k j}-\varphi_{k i}\right)
$$

Por (3.6) seque que

$$
\varphi_{i j}+\varphi_{j k}+\varphi_{k i}=2 \pi m
$$

para algum $m \in \mathbb{Z}$, ou seja,

$$
\varphi_{i j}-2 \pi m=-\varphi_{j k}-\varphi_{k i}=\varphi_{k j}-\varphi_{k i}
$$

Assim,

$$
\begin{aligned}
\xi_{j}-\xi_{i} & =\frac{1}{2 \pi} \sum_{k} \rho_{k} d\left(\varphi_{i j}-2 \pi m\right) \\
& =\frac{1}{2 \pi} \sum_{k} \rho_{k} d \varphi_{i j} \\
& =\frac{1}{2 \pi} d \varphi_{i j} .
\end{aligned}
$$

Do Lema 3.33 segue-se que $d \xi_{i}=d \xi_{j}$ em $U_{i} \cap U_{j}$. Assim, podemos definir uma 2-forma global e tal que restrita a cada $U_{i}$ é $d \xi_{i}$. Esta forma e é fechada, mas não necessariamente exata, pois $\xi_{i}$ geralmente não pode ser definida globalmente. A classe de cohomologia de e em $H^{2}(M)$ é chamada classe de Euler do fibrado orientado E. As vezes escrevemos e $(E)$ em vez de e.

Proposição 3.34. A classe de cohomologia de e é independente da escolha de $\xi$ na construção acima.

Demonstração. Se $\left\{\bar{\xi}_{i}\right\}$ é uma escolha diferente de 1-formas tais que

$$
\frac{1}{2 \pi} d \varphi_{i j}=\bar{\xi}_{j}-\bar{\xi}_{i}=\xi_{j}-\xi_{i}
$$

então,

$$
\bar{\xi}_{j}-\xi_{j}=\bar{\xi}_{i}-\xi_{i}=\xi
$$

e $\xi$ é uma 1-forma global. Logo,

$$
d \bar{\xi}_{i}-d \xi_{i}=d \xi \Longrightarrow[\overline{\mathrm{e}}]=[\mathrm{e}]
$$

Por (3.5) e (3.7) temos

$$
d \theta_{j}=d \theta_{i}+\pi^{*} d \varphi_{i j} \Longrightarrow d \theta_{j}=d \theta_{i}+2 \pi\left(\pi^{*}\left(\xi_{j}-\xi_{i}\right)\right) \Longrightarrow \frac{d \theta_{j}}{2 \pi}-\pi^{*} \xi_{j}=\frac{d \theta_{i}}{2 \pi}-\pi^{*} \xi_{i}
$$


em $\left.E^{0}\right|_{U_{i} \cap U_{j}}$. Assim, obtemos uma 1-forma global $\psi$ em $E^{0}$, a forma angular global, cuja restrição a cada fibra é a forma angular $(1 / 2 \pi) d \theta$, isto é, se $i_{p}: \mathbb{R}^{2} \rightarrow E$ é a inclusão ortogonal da fibra sob $p$, então, $i_{p}^{*} \psi=(1 / 2 \pi) d \theta$. A forma angular global não é fechada

$$
d \psi=d\left(\frac{d \theta_{i}}{2 \pi}-\pi^{*} \xi_{i}\right)=-\pi^{*} d \xi_{i}=-\pi^{*} d \xi_{j}
$$

no entanto, pela equação acima segue que

$$
d \psi=-\pi^{*} \mathrm{e}
$$

No caso quando $\pi: E \rightarrow M$ é um fibrado vetorial onde cada fibra é uma esfera, também é possível definir uma função ângulo $\psi$ tal que a igualdade (3.8) se verifica.

Observação 3.35. Quando E é um produto, isto é, $E=M \times \mathbb{R}^{2}, \psi$ pode ser tomada como o pullback de $(1 / 2 \pi) d \theta$ sob a projeção $E^{0}=M \times\left(\mathbb{R}^{2}-\{0\}\right) \rightarrow \mathbb{R}^{2}-\{0\}$. Nesse caso $\psi$ é fechada, logo, $\mathrm{e}=0$.

Proposição 3.36. Seja E um fibrado orientado, onde cada fibra é uma esfera $S^{n}, n \geq 1$. Se E tem uma seção que nunca se anula, então, a classe de Euler é identicamente nula.

Proposição 3.37. O número de Euler de uma variedade compacta orientável, definido por $\int_{M} \mathrm{e}(T M)$, é igual a característica de Euler $\chi(M)=\sum(-1)^{q} \operatorname{dim} H^{q}$.

Exemplo 3.38. A característica de Euler de uma n-esfera $S^{n}$ é:

$$
\chi\left(S^{n}\right)=1+(-1)^{n}= \begin{cases}2 & \text { se } n \text { é par } \\ 0 & \text { se n é ímpar. }\end{cases}
$$

Assim, pela Proposição 3.37 se n é par temos

$$
\int_{S^{n}} \mathrm{e}\left(T S^{n}\right)=2 \Longrightarrow \mathrm{e}\left(T S^{n}\right) \neq 0
$$

Seja $n=2 k-1$. Então existe uma seção do fibrado unitário de $S^{2 n-2} \subset \mathbb{R}^{2 n}$ dado por

$$
Y(x)=\left(x_{2},-x_{1}, x_{4},-x_{3}, \ldots, x_{2 n},-x_{2 n-1}\right) \in T_{1} S^{2 n-1}, x \in S^{2 n-1}
$$

que nunca se anula. Logo, pela Proposição 3.36 segue-seque e $\left(T_{1} S^{2 n-1}\right)=0$.

\subsection{Sequência de Gysin}

Um tipo especial de sequência, a sequência espectral, de um fibrado é essencialmente um modo de descrever as relações algébricas entre a cohomologia do espaço base, das fibras e do espaço do fibrado total. Em certas situações especiais a sequência espectral se reduz a uma sequência exata longa. Um desses casos especiais é a cohomologia de um fibrado cujas fibras são esferas. O resultado é uma sequência chamada sequência de Gysin.

Teorema 3.39. Seja $\pi: E \rightarrow M$ um fibrado vetorial orientado com fibra $S^{k}$. Então, existe uma sequência exata longa

$$
\cdots \longrightarrow H^{n}(E) \stackrel{\pi_{*}}{\longrightarrow} H^{n-k}(M) \stackrel{\wedge \mathrm{e}}{\longrightarrow} H^{n+1}(M) \stackrel{\pi^{*}}{\longrightarrow} H^{n+1}(E) \longrightarrow \cdots
$$

em que as aplicações $\pi_{*}, \wedge \mathrm{e} e \pi^{*}$ são integração ao longo da fibra, multiplicação pela classe de Euler e o pullback natural, respectivamente. 


\subsection{Aplicações}

Sejam $M=S^{n}, M=\mathbb{C} P^{n}$ ou $M=\mathbb{H} P^{n}$. Então $M$ com a métrica canônica forma uma variedade Riemanniana onde todas as geodésicas são fechadas de mesmo período $2 \pi$ (a menos de reparametrização), isto é, $M$ é uma $C_{2 \pi}$-variedade.

Observação 3.40. Seja $(M, g)$ uma variedade Riemannina e $C=T_{1} M / S^{1}$ como definido em (1.30), onde $S^{1}=\mathbb{R} / 2 \pi \mathbb{Z}$. Suponha que $\left(x_{1}, v_{1}\right) \sim\left(x_{2}, v_{2}\right)$, então

$$
\left(x_{2}, v_{2}\right)=\left(\gamma_{x_{1}}^{v_{1}}(s), \dot{\gamma}_{x_{1}}^{v_{1}}(s)\right)
$$

para algum $s \in S^{1}$, ou seja,

$$
\left(\gamma_{x_{2}}^{v_{2}}(0), \dot{\gamma}_{x_{2}}^{v_{2}}(0)\right)=\left(\gamma_{x_{1}}^{v_{1}}(s), \dot{\gamma}_{x_{1}}^{v_{1}}(s)\right)
$$

Logo, pela unicidade das geodésicas obtemos

$$
\gamma_{x_{1}}^{v_{1}}=\gamma_{x_{2}}^{v_{2}}
$$

Como $\left(x_{1}, v_{1}\right)$ e $\left(x_{2}, v_{2}\right)$ são elementos arbitrários de uma classe de equivalência, então podemos identificar $[(x, v)]$ com a geodésica $\Gamma(s):=(\gamma(2 \pi s), \dot{\gamma}(2 \pi s)) \in T_{1} M$ onde para todo $s \in S^{1}$ tem-se $\Gamma(s) \sim(x, v)$, ou seja,

$$
[(x, v)]=\left\{(\gamma(s), \dot{\gamma}(s)) ; s \in S^{1}\right\} .
$$

Dessa forma, podemos definir uma aplicação $j: C \rightarrow T_{1} M$ por $j([(x, v)])=(\gamma(0), \dot{\gamma}(0))$. Assim, pelo Lema 3.22 obtemos uma aplicação $j^{*}: H^{k}\left(T_{1} M\right) \rightarrow H^{k}(C)$. Como $\pi \circ j=I d$ segue por um argumento análogo ao da demonstração da Proposição 3.23 que $j^{*} \circ \pi^{*}=I d$.

Lema 3.41. Sejam $M=S^{2 n}$ e $C=T_{1} S^{2 n} / S^{1}$. Então, os números de Betti de C são:

$$
b_{i}(C)=\left\{\begin{array}{l}
1, \quad \text { se i é par e } 0 \leq i \leq 4 n-2 \\
0, \quad \text { caso contrário. }
\end{array}\right.
$$

Demonstração. Tome a fibração $S^{2 n-1} \rightarrow T_{1} S^{2 n} \rightarrow S^{2 n}$. Então, a sequência de Gysin associada a esta fibração é:

$$
\cdots \longrightarrow H^{p}\left(T_{1} S^{2 n}\right) \stackrel{\pi_{*}}{\longrightarrow} H^{p-(2 n-1)}\left(S^{2 n}\right) \stackrel{\wedge \mathrm{e}}{\longrightarrow} H^{p+1}\left(S^{2 n}\right) \stackrel{\pi^{*}}{\longrightarrow} H^{p+1}\left(T_{1} S^{2 n}\right) \longrightarrow \cdots
$$

Observe que como $T_{1} S^{2 n}$ é conexo temos $H^{0}\left(T_{1} S^{2 n}\right) \cong \mathbb{R}$. Além disso, sabemos que

$$
H^{k}\left(S^{n}\right)= \begin{cases}\mathbb{R}, & \text { se } k=0 \text { ou } k=n \\ 0, & \text { caso contrário. }\end{cases}
$$

Assim, como a sequência de Gysin é uma sequência exata longa e sabemos as cohomologias de $S^{2 n}$, podemos calcular as cohomologias de $T_{1} S^{2 n}$.

- Para $0<p \leq 2 n-2$ temos $p-(2 n-1)<0$. Logo, $H^{p-(2 n-1)}\left(S^{2 n}\right)=0$. Usando,

$$
\underbrace{H^{p}\left(S^{2 n}\right)}_{=0} \rightarrow H^{p}\left(T_{1} S^{2 n}\right) \rightarrow \underbrace{H^{p-(2 n-1)}\left(S^{2 n}\right)}_{=0} \rightarrow H^{p+1}\left(S^{2 n}\right)
$$

concluímos que $H^{p}\left(T_{1} S^{2 n}\right)=0$ para todo $0<p \leq 2 n-2$.

- Se $p=2 n-1$ temos

$$
0 \longrightarrow H^{2 n-1}\left(T_{1} S^{2 n}\right) \stackrel{\pi_{*}}{\longrightarrow} \underbrace{H^{0}\left(S^{2 n}\right)}_{\cong \mathbb{R}} \stackrel{\wedge \mathrm{e}}{\longrightarrow} \underbrace{H^{2 n}\left(S^{2 n}\right)}_{\cong \mathbb{R}} \stackrel{\pi^{*}}{\longrightarrow} H^{2 n}\left(T_{1} S^{2 n}\right) \longrightarrow \underbrace{H^{1}\left(S^{2 n}\right)}_{=0}
$$


Como a classe de Euler de uma esfera de dimensão par é diferente de zero, como vimos no exemplo 3.38, e a $\operatorname{dim} H_{0}\left(S^{2 n}\right)=1$ segue-se que a aplicação $\wedge$ e $: H^{0}\left(S^{2 n}\right) \rightarrow H^{2 n}\left(S^{2 n}\right)$ é injetora. Logo,

$$
H^{2 n-1}\left(T_{1} S^{2 n}\right) \cong \operatorname{Im}\left(\pi_{*}\right)=\operatorname{ker}(\wedge \mathrm{e})=0 \text { e } \mathbb{R} \cong \operatorname{Im}(\wedge \mathrm{e})=\operatorname{ker}\left(\pi^{*}\right) .
$$

Além disso, como

$$
\operatorname{dim} \operatorname{Im}\left(\pi^{*}\right)+\operatorname{dim} \operatorname{ker}\left(\pi^{*}\right)=\operatorname{dim} H^{2 n}\left(S^{2 n}\right)=1 \Longrightarrow \operatorname{dim} \operatorname{Im}\left(\pi^{*}\right)=0 \Longrightarrow \operatorname{Im}\left(\pi^{*}\right)=0
$$

temos $H^{2 n}\left(T_{1} S^{2 n}\right)=\operatorname{Im}\left(\pi^{*}\right)=0$.

- Se $2 n+1 \leq p \leq 4 n-2$ segue que $H^{p-(2 n-1)}\left(S^{2 n}\right)=0$ pois $2 \leq p-(2 n-1) \leq 2 n-1$. Então, como

$$
\underbrace{H^{p}\left(S^{2 n}\right)}_{=0} \rightarrow H^{p}\left(T_{1} S^{2 n}\right) \rightarrow \underbrace{H^{p-(2 n-1)}\left(S^{2 n}\right)}_{=0} \rightarrow H^{p+1}\left(S^{2 n}\right)
$$

concluímos que $H^{p}\left(T_{1} S^{2 n}\right)=0$.

- Se $p=4 n-1$ temos

$$
0 \rightarrow H^{4 n-1}\left(T_{1} S^{2 n}\right) \rightarrow \underbrace{H^{2 n}\left(S^{2 n}\right)}_{\cong \mathbb{R}} \rightarrow \underbrace{H^{4 n}\left(S^{2 n}\right)}_{=0}
$$

Assim, $H^{4 n-1}\left(T_{1} S^{2 n}\right) \cong H^{2 n}\left(S^{2 n}\right) \cong \mathbb{R}$

Resumindo, temos

$$
H^{p}\left(T_{1} S^{2 n}\right)= \begin{cases}\mathbb{R}, & \text { se } p=0 \text { ou } p=4 n-1 \\ 0, & \text { caso contrário. }\end{cases}
$$

Tomando agora a fibração $S^{1} \rightarrow T_{1} S^{2 n} \rightarrow C$ podemos calcular as cohomologias de $C$ a partir das cohomologias de $T_{1} S^{2 n}$. A sequência de Gysin associada a esta fibração é:

$$
\cdots \longrightarrow H^{p}\left(T_{1} S^{2 n}\right) \stackrel{\pi_{*}}{\longrightarrow} H^{p-1}(C) \stackrel{\wedge \mathrm{e}}{\longrightarrow} H^{p+1}(C) \stackrel{\pi^{*}}{\longrightarrow} H^{p+1}\left(T_{1} S^{2 n}\right) \longrightarrow \cdots
$$

onde e $\in H^{2}(C)$ é a classe de Euler do $S^{1}$-fibrado sobre $C$ induzido pela fibração $S^{1} \rightarrow T_{1} S^{2 n} \rightarrow C$. Observe novamente que como $C$ é conexo temos $H^{0}(C) \cong \mathbb{R}$.

- $p=0$ temos

$$
H^{0}\left(T_{1} S^{2 n}\right) \rightarrow \underbrace{H^{-1}(C)}_{=0} \rightarrow H^{1}(C) \rightarrow \underbrace{H^{1}\left(T_{1} S^{2 n}\right)}_{=0} \rightarrow \cdots
$$

Logo, $H^{1}(C)=0$.

- Se $p=1$ temos

$$
0 \rightarrow \underbrace{H^{1}\left(T_{1} S^{2 n}\right)}_{=0} \rightarrow H^{0}(C) \rightarrow H^{2}(C) \rightarrow \underbrace{H^{2}\left(T_{1} S^{2 n}\right)}_{=0} \rightarrow H^{1}(C) \rightarrow H^{3}(C) \rightarrow \underbrace{H^{3}\left(T_{3} S^{2 n}\right)}_{=0}
$$

$\operatorname{Logo}, H^{2}(C) \cong H^{0} \cong \mathbb{R}$ e $H^{3}(C) \cong H^{1}(C)=0$.

- De forma indutiva, para $0 \leq k \leq 2 n-3$ temos

$$
\underbrace{H^{2 k+1}\left(T_{1} S^{2 n}\right)}_{=0} \rightarrow H^{2 k}(C) \rightarrow H^{2 k+2}(C) \rightarrow \underbrace{H^{2 k+2}\left(T_{1} S^{2 n}\right)}_{=0} \rightarrow H^{2 k+1}(C) \rightarrow H^{2 k+3}(C) \rightarrow \underbrace{H^{2 k+3}\left(T_{3} S^{2 n}\right)}_{=0}
$$

Logo, $H^{2 k+2}(C) \cong H^{2 k} \cong \mathbb{R}$ e $H^{2 k+3}(C) \cong H^{2 k+1}(C)=0$, ou seja,

$$
H^{p}(C) \cong \begin{cases}\mathbb{R}, & \text { se pé par e } 0 \leq p \leq 4 n-3 \\ 0, & \text { se pé ímpar e } 0 \leq p \leq 4 n-3\end{cases}
$$


- Se $p=4 n-3$ temos

$$
\underbrace{H^{4 n-3}\left(T_{1} S^{2 n}\right)}_{=0} \rightarrow H^{4 n-4}(C) \rightarrow H^{4 n-2}(C) \rightarrow \underbrace{H^{4 n-2}\left(T_{1} S^{2 n}\right)}_{=0}
$$

$\operatorname{Logo}, H^{4 n-2}(C) \cong H^{4 n-4}(C) \cong \mathbb{R}$.

- Seja $p=4 n-2$. Como $\operatorname{dim} C=4 n-2$ temos $\Omega^{4 n}(C)=0$ e isto implica que $H^{4 n}(C)=0$. Assim, temos

$$
\underbrace{H^{4 n-3}(C)}_{=0} \longrightarrow H^{4 n-1}(C) \stackrel{\pi^{*}}{\longrightarrow} \underbrace{H^{4 n-1}\left(T_{1} S^{2 n}\right)}_{\cong \mathbb{R}} \stackrel{\pi_{*}}{\longrightarrow} \underbrace{H^{4 n-2}(C)}_{\cong \mathbb{R}} \longrightarrow \underbrace{H^{4 n}(C)}_{=0}
$$

$\operatorname{Logo}, H^{4 n-1}(C) \cong \operatorname{Im}\left(\pi^{*}\right)=\operatorname{ker}\left(\pi_{*}\right)=0$.

Portanto, $b_{p}(C)=1$ se $p$ é um número par tal que $0 \leq p \leq 4 n-2$ e zero nos outros casos.

Lema 3.42. Sejam $M=S^{2 n+1}$ e $C=T_{1} S^{2 n+1} / S^{1}$. Então, os números de Betti de C são:

$$
b_{i}(C)= \begin{cases}1, & \text { se i é par e } 0 \leq i \leq 2 n-2 \\ 2, & \text { se } i=2 n \\ 1, & \text { se i é par e } 2 n+2 \leq i \leq 4 n \\ 0, & \text { caso contrário. }\end{cases}
$$

Demonstração. Tome a fibração $S^{2 n} \rightarrow T_{1} S^{2 n+1} \rightarrow S^{2 n+1}$. A sequência de Gysin associada a esta fibração é:

$\cdots \longrightarrow H^{p}\left(T_{1} S^{2 n+1}\right) \stackrel{\pi_{*}}{\longrightarrow} H^{p-(2 n)}\left(S^{2 n+1}\right) \stackrel{\wedge \mathrm{e}}{\longrightarrow} H^{p+1}\left(S^{2 n+1}\right) \stackrel{\pi^{*}}{\longrightarrow} H^{p+1}\left(T_{1} S^{2 n+1}\right) \longrightarrow \cdots$

Como no Lema 3.41, temos $H^{0}\left(T_{1} S^{2 n+1}\right) \cong \mathbb{R}, H^{0}\left(S^{2 n+1}\right) \cong H^{2 n+1}\left(S^{2 n+1}\right) \cong \mathbb{R}$ e $H^{k}\left(S^{2 n+1}\right)=0$ quando $k \neq 0$ e $k \neq 2 n+1$.

- Se $0<p \leq 2 n-1$ temos $H^{p-2 n}\left(S^{2 n+1}\right)=0$ pois $p-2 n<0$. Logo, nesses casos $H^{p}\left(S^{2 n+1}\right)=0=H^{p-2 n}\left(S^{2 n+1}\right)$ e pela parte da sequência

$$
\underbrace{H^{p}\left(S^{2 n+1}\right)}_{=0} \rightarrow H^{p}\left(T_{1} S^{2 n+1}\right) \rightarrow \underbrace{H^{p-2 n}\left(S^{2 n+1}\right)}_{=0} \rightarrow H^{p+1}\left(S^{2 n+1}\right)
$$

obtemos $H^{p}\left(T_{1} S^{2 n+1}\right)=0$.

- Se $p=2 n$ temos

$$
0 \longrightarrow H^{2 n}\left(T_{1} S^{2 n+1}\right) \stackrel{\pi_{*}}{\longrightarrow} \underbrace{H^{0}\left(S^{2 n+1}\right)}_{\cong \mathbb{R}} \stackrel{\wedge \mathrm{e}}{\longrightarrow} \underbrace{H^{2 n+1}\left(S^{2 n+1}\right)}_{\cong \mathbb{R}} \stackrel{\pi^{*}}{\longrightarrow} H^{2 n+1}\left(T_{1} S^{2 n+1}\right) \longrightarrow \underbrace{H^{1}\left(S^{2 n+1}\right)}_{=0}
$$

Como no Exemplo 3.38 vimos que a classe de Euler de uma esfera de dimensão ímpar é zero, então

$$
0=\operatorname{Im}(\wedge \mathrm{e})=\operatorname{ker}\left(\pi^{*}\right)
$$

Assim,

$$
\mathbb{R} \cong \operatorname{Im}\left(\pi^{*}\right) \cong H^{2 n+1}\left(T_{1} S^{2 n+1}\right)
$$

$\mathrm{e}$

$$
\mathbb{R} \cong \operatorname{ker}(\wedge \mathrm{e})=\operatorname{Im}\left(\pi_{*}\right) \cong H^{2 n}\left(T_{1} S^{2 n+1}\right) .
$$

- Se $2 n+2 \leq p \leq 4 n$ temos $H^{p-2 n}\left(S^{2 n+1}\right)$ pois $2 \leq p-2 n \leq 2 n$. Logo,

$$
\underbrace{H^{p}\left(S^{2 n+1}\right)}_{=0} \rightarrow H^{p}\left(T_{1} S^{2 n+1}\right) \rightarrow \underbrace{H^{p-2 n}\left(S^{2 n+1}\right)}_{=0} \rightarrow H^{p+1}\left(S^{2 n+1}\right)
$$

e isto implica que $H^{p}\left(T_{1} S^{2 n+1}\right)=0$.

- Se $p=4 n+1$ temos

$$
0 \rightarrow H^{4 n+1}\left(T_{1} S^{2 n+1}\right) \rightarrow \underbrace{H^{2 n+1}\left(S^{2 n+1}\right)}_{\cong \mathbb{R}} \rightarrow \underbrace{H^{4 n+2}\left(S^{2 n+1}\right)}_{=0} \rightarrow H^{4 n+2}\left(T_{1} S^{2 n+1}\right)
$$


$\operatorname{Logo}, H^{4 n+1}\left(T_{1} S^{2 n+1}\right) \cong H^{2 n+1}\left(S^{2 n+1}\right) \cong \mathbb{R}$.

Resumindo:

$$
H^{p}\left(T_{1} S^{2 n+1}\right)= \begin{cases}\mathbb{R}, & \text { se } p=0,2 n, 2 n+1,4 n+1 \\ 0, & \text { caso contrário. }\end{cases}
$$

Tomando a fibração $S^{1} \rightarrow T_{1} S^{2 n+1} \rightarrow C$, obtemos a sequência de Gysin

$$
\cdots \longrightarrow H^{p}\left(T_{1} S^{2 n+1}\right) \stackrel{\pi_{*}}{\longrightarrow} H^{p-1}(C) \stackrel{\wedge \mathrm{e}}{\longrightarrow} H^{p+1}(C) \stackrel{\pi^{*}}{\longrightarrow} H^{p+1}\left(T_{1} S^{2 n+1}\right) \longrightarrow \cdots
$$

- Se $p=0$ temos

$$
H^{0}\left(T_{1} S^{2 n+1}\right) \rightarrow \underbrace{H^{-1}(C)}_{=0} \rightarrow H^{1}(C) \rightarrow \underbrace{H^{1}\left(T_{1} S^{2 n+1}\right)}_{=0}
$$

ou seja, $H^{1}(C)=0$.

- Se $p=1$ temos

$$
\underbrace{H^{1}\left(T_{1} S^{2 n+1}\right)}_{=0} \rightarrow H^{0}(C) \rightarrow H^{2}(C) \rightarrow \underbrace{H^{2}\left(T_{1} S^{2 n+1}\right)}_{=0} \rightarrow H^{1}(C) \rightarrow H^{3}(C) \rightarrow \underbrace{H^{3}\left(T_{1} S^{2 n+1}\right)}_{=0}
$$

$\operatorname{Logo}, H^{2}(C) \cong H^{0}(C) \cong \mathbb{R}$ e $H^{3}(C) \cong H^{1}(C)=0$.

- Indutivamente, se $0 \leq k \leq n-2$ temos $1 \leq 2 k+1<2 k+2<2 k+3 \leq 2 n-1$ e disso segue que

$$
\underbrace{H^{2 k+1}\left(T_{1} S^{2 n+1}\right)}_{=0} \rightarrow H^{2 k}(C) \rightarrow H^{2 k+2}(C) \rightarrow \underbrace{H^{2 k+2}\left(T_{1} S^{2 n+1}\right)}_{=0} \rightarrow H^{2 k+1}(C) \rightarrow H^{2 k+3}(C) \rightarrow \underbrace{H^{2 k+3}\left(T_{1} S^{2 n+1}\right)}_{=0}
$$

$\operatorname{Logo}, H^{2 k+2}(C) \cong H^{2 k}(C) \cong \mathbb{R}$ e $H^{2 k+3}(C) \cong H^{2 k+1}(C)=0$.

- Se $p=2 n-1$ temos

$$
\underbrace{H^{2 n-1}\left(T_{1} S^{2 n+1}\right)}_{=0} \rightarrow \underbrace{H^{2 n-2}(C)}_{\cong \mathbb{R}} \stackrel{\wedge \mathrm{e}}{\rightarrow} H^{2 n}(C) \stackrel{\pi^{*}}{\rightarrow} \underbrace{H^{2 n}\left(T_{1} S^{2 n+1}\right)}_{\cong \mathbb{R}} \rightarrow \underbrace{H^{2 n-1}(C)}_{=0} \rightarrow H^{2 n+1}(C) \rightarrow \underbrace{H^{2 n+1}\left(T_{1} S^{2 n+1}\right)}_{\cong \mathbb{R}}
$$

Pela observação 3.40 obtemos uma aplicação $j^{*}: H^{k}\left(T_{1} S^{2 n+1}\right) \rightarrow H^{k}(C)$ tal que $j^{*} \circ \pi^{*}=I d$. Assim, pelo Lema de Splitting segue-se que

$$
H^{2 n}(C) \cong H^{2 n-2}(C) \oplus H^{2 n}\left(T_{1} S^{2 n+1}\right) \cong \mathbb{R} \oplus \mathbb{R} .
$$

Tome agora a seguinte parte da sequência de Gysin:

$$
0 \rightarrow H^{2 n+1}(C) \stackrel{\pi^{*}}{\rightarrow} \underbrace{H^{2 n+1}\left(T_{1} S^{2 n+1}\right)}_{\cong \mathbb{R}} \stackrel{\pi_{*}}{\rightarrow} \underbrace{H^{2 n}(C)}_{\cong \mathbb{R} \oplus \mathbb{R}} \stackrel{\wedge \mathrm{e}}{\rightarrow} H^{2 n+2}(C) \rightarrow \underbrace{H^{2 n+2}\left(T_{1} S^{2 n+1}\right)}_{=0}
$$

Como vimos na equação (3.9)

$$
H^{2 n+1}\left(T_{1} S^{2 n+1}\right) \stackrel{\tilde{\pi}^{*}}{\cong} H^{2 n+1}\left(S^{2 n+1}\right) \cong \mathbb{R}
$$

onde $\tilde{\pi}^{*}:=\pi^{*}: H^{2 n+1}\left(T_{1} S^{2 n+1}\right) \rightarrow H^{2 n+1}\left(S^{2 n+1}\right)$. Então, $H^{2 n+1}\left(T_{1} S^{2 n+1}\right)$ é gerado por um elemento $\tilde{\pi}^{*} \phi$ que é o pullback da forma volume $\phi$ em $\Omega^{2 n+1}\left(S^{2 n+1}\right)$. Assim, se $t^{1}, \ldots, t^{2 n+1}$ são coordenadas em $S^{2 n+1}$ segue-se que $\widetilde{\pi}^{*} \phi=d t^{1} \wedge \ldots \wedge d t^{2 n+1} \mathrm{e}$

$$
\begin{aligned}
\pi_{*}\left(\tilde{\pi}^{*} \phi\right) & =d t^{1} \wedge \ldots \wedge \widehat{d t^{i}} \wedge \ldots \wedge d t^{2 n+1} \int_{S^{1}} d x^{i} \\
& =2 \pi d t^{1} \wedge \ldots \wedge \widehat{d t^{i}} \wedge \ldots \wedge d t^{2 n+1} \\
& \neq 0
\end{aligned}
$$

Logo, $\operatorname{ker}\left(\pi_{*}\right)=0$, ou seja, $\pi_{*}$ é injetora. Disso segue-se que $H^{2 n+1}(C) \cong \operatorname{Im}\left(\pi^{*}\right)=\operatorname{ker}\left(\pi_{*}\right)=0$ e $1=$ $\operatorname{dim} \operatorname{Im}\left(\pi_{*}\right)=\operatorname{dim} \operatorname{ker}(\wedge \mathrm{e})$. Assim,

$$
2=\operatorname{dim} H^{2 n}(C)=\operatorname{dim} \operatorname{Im}(\wedge \mathrm{e})+1 \Longrightarrow \operatorname{dim} \operatorname{Im}(\wedge \mathrm{e})=1 .
$$

Logo, $b_{2 n+2}(C)=\operatorname{dim} H^{2 n+2}(C)=\operatorname{dim} \operatorname{Im}(\wedge \mathrm{e})=1$. 
- Se $n+1 \leq k \leq 2 n-1$ temos

$$
2 n+2 \leq 2 k<2 k+1<2 k+2 \leq 4 n-2 .
$$

Logo,

$$
\underbrace{H^{2 k}\left(T_{1} S^{2 n+1}\right)}_{=0} \rightarrow H^{2 k-1}(C) \rightarrow H^{2 k+1}(C) \rightarrow \underbrace{H^{2 k+1}\left(T_{1} S^{2 n+1}\right)}_{=0} \rightarrow H^{2 k}(C) \rightarrow H^{2 k+2}(C) \rightarrow \underbrace{H^{2 k+2}\left(T_{1} S^{2 n+1}\right)}_{=0}
$$

e de forma indutiva obtemos: $H^{2 k+1}(C) \cong H^{2 k-1}(C)=0$ e $H^{2 k+2}(C) \cong H^{2 k}(C) \cong \mathbb{R}$.

- Se $p=4 n$ temos

$$
\underbrace{H^{4 n}\left(T_{1} S^{2 n+1}\right)}_{=0} \rightarrow \underbrace{H^{4 n-1}(C)}_{=0} \rightarrow H^{4 n+1}(C) \stackrel{\pi^{*}}{\rightarrow} \underbrace{H^{4 n+1}\left(T_{1} S^{2 n+1}\right)}_{\cong \mathbb{R}} \stackrel{\pi_{*}}{\rightarrow} \underbrace{H^{4 n}(C)}_{\cong \mathbb{R}} \stackrel{\wedge e}{\rightarrow} H^{4 n+2}(C) \rightarrow \underbrace{H^{4 n+2}\left(T_{1} S^{2 n+1}\right)}_{=0}
$$

Como $H^{4 n+1}\left(T_{1} S^{2 n+1}\right)$ é gerado pela forma volume de $T_{1} S^{2 n+1}$ temos $\pi_{*} \neq 0$. Logo, como $\operatorname{Im}\left(\pi_{*}\right) \subset H^{4 n}(C)$ temos

$$
1 \leq \operatorname{dim} \operatorname{Im}\left(\pi_{*}\right) \leq H^{4 n}(C)=1 \Longrightarrow \operatorname{dim} \operatorname{Im}\left(\pi_{*}\right)=1
$$

e $\operatorname{dim} \operatorname{ker}\left(\pi_{*}\right)=\operatorname{dim} H^{4 n+1}\left(T_{1} S^{2 n+1}\right)-\operatorname{dim} \operatorname{Im}\left(\pi_{*}\right)=0$. Assim,

$$
H^{4 n+1}(C) \cong \operatorname{Im}\left(\pi^{*}\right)=\operatorname{ker}\left(\pi_{*}\right)=0 .
$$

Além disso, como $1=\operatorname{dim} \operatorname{Im}\left(\pi_{*}\right)=\operatorname{dim} \operatorname{ker}(\wedge \mathrm{e}) \mathrm{e} \operatorname{dim} H^{4 n}(C)=1$ temos

$$
0=\operatorname{Im}(\wedge \mathrm{e}) \cong H^{4 n+2}(C) .
$$

Portanto, $b_{k}(C)=1$ se $k$ é um inteiro par tal que $0 \leq k \leq 4 n$ e $k \neq 2 n, b_{2 n}(C)=2$ e $b_{k}(C)=0$ nos outros casos.

Lema 3.43. Sejam $M=\mathbb{C} P^{n}$ e $C=T_{1} \mathbb{C} P^{n} / S^{1}$. Então, os números de Betti de $C$ são:

$$
b_{0}(C)=1, b_{2}(C)=2, b_{4}(C)=3, \ldots, b_{2 n-2}(C)=n=b_{2 n}, b_{2 n+1}(C)=n-1, \ldots, b_{4 n-2}(C)=1
$$

$e b_{p}=0$ se pé ímpar.

Demonstração. Demonstraremos o caso $n=2 m$, o caso $n$ ímpar é análogo. Sabemos que

$$
H^{k}\left(\mathbb{C} P^{n}\right) \cong \begin{cases}\mathbb{R}, & \text { se k é par e } 0 \leq k \leq 2 n \\ 0, & \text { caso contrário. }\end{cases}
$$

Tome a fibração $S^{4 m-1} \rightarrow T_{1} \mathbb{C} P^{2 m} \rightarrow \mathbb{C} P^{2 m}$. A sequência de Gysin associada a esta fibração é:

$\cdots \longrightarrow H^{p}\left(T_{1} \mathbb{C} P^{2 m}\right) \stackrel{\pi_{*}}{\longrightarrow} H^{p-(4 m-1)}\left(\mathbb{C} P^{2 m}\right) \stackrel{\wedge \mathrm{e}}{\longrightarrow} H^{p+1}\left(\mathbb{C} P^{2 m}\right) \stackrel{\pi^{*}}{\longrightarrow} H^{p+1}\left(T_{1} \mathbb{C} P^{2 m}\right) \longrightarrow \cdots$

Como $T_{1} \mathbb{C} P^{2 m}$ é conexo temos $H^{0}\left(T_{1} \mathbb{C} P^{2 m}\right) \cong \mathbb{R}$.

- Para $1 \leq p \leq 4 m-2$ temos $p-(4 m-1)<0$. Logo $H^{p-(4 m-1)}\left(\mathbb{C} P^{2 m}\right)=0$ e de

$$
\underbrace{H^{(p-1)-(4 m-1)}\left(\mathbb{C} P^{2 m}\right)}_{=0} \rightarrow H^{p}\left(\mathbb{C} P^{2 m}\right) \rightarrow H^{p}\left(T_{1} \mathbb{C} P^{2 m}\right) \rightarrow \underbrace{H^{p-(4 m-1)}\left(\mathbb{C} P^{2 m}\right)}_{=0}
$$

segue que $H^{p}\left(\mathbb{C} P^{2 m}\right) \cong H^{p}\left(T_{1} \mathbb{C} P^{2 m}\right)$. Assim,

$$
H^{p}\left(T_{1} \mathbb{C} P^{n}\right) \cong \begin{cases}\mathbb{R}, & \text { se p é par e } 1 \leq p \leq 4 m-2 \\ 0, & \text { se p é ímpar e } 1 \leq p \leq 4 m-2 .\end{cases}
$$

- Se $p=4 m-1$ temos

$$
0 \rightarrow H^{4 m-1}\left(T_{1} \mathbb{C} P^{2 m}\right) \stackrel{\pi_{\star}}{\rightarrow} \underbrace{H^{0}\left(\mathbb{C} P^{2 m}\right)}_{\cong \mathbb{R}} \stackrel{\wedge e}{\rightarrow} \underbrace{H^{4 m}\left(\mathbb{C} P^{2 m}\right)}_{\cong \mathbb{R}} \stackrel{\pi^{*}}{\rightarrow} H^{4 m}\left(T_{1} \mathbb{C} P^{2 m}\right) \rightarrow \underbrace{H^{1}\left(\mathbb{C} P^{2 m}\right)}_{=0}
$$


Como a característica de Euler de $\mathbb{C} P^{n}$ é $n+1$ então pela Proposição 3.37 temos

$$
\int_{\mathbb{C} P 2 m} \mathrm{e}=2 m+1 \Longrightarrow \mathrm{e} \neq 0
$$

Assim, como $\operatorname{dim} \operatorname{Im}(\wedge \mathrm{e}) \geq 1 \mathrm{e} \operatorname{Im}(\wedge \mathrm{e}) \subset H^{4 m}\left(\mathbb{C} P^{2 m}\right)$ temos

$$
1 \leq \operatorname{dim} \operatorname{Im}(\wedge \mathrm{e}) \leq \operatorname{dim} H^{4 m}\left(\mathbb{C} P^{2 m}\right)=1 \Longrightarrow \operatorname{dim} \operatorname{Im}(\wedge \mathrm{e})=1
$$

e disso segue que

- $\operatorname{dim} \operatorname{ker}(\wedge \mathrm{e})=\operatorname{dim} H^{0}\left(\mathbb{C} P^{2 m}\right)-\operatorname{dim} \operatorname{Im}(\wedge \mathrm{e})=1-1=0$

- $1=\operatorname{dim} \operatorname{Im}(\wedge \mathrm{e})=\operatorname{dim} \operatorname{ker}\left(\pi^{*}\right)$

- $\operatorname{dim} \operatorname{Im}\left(\pi^{*}\right)=\operatorname{dim} H^{4 m}\left(\mathbb{C} P^{2 m}\right)-\operatorname{dim} \operatorname{ker}\left(\pi^{*}\right)=1-1=0$

Assim,

$$
H^{4 m-1}\left(T_{1} \mathbb{C} P^{2 m}\right) \cong \operatorname{Im}\left(\pi_{*}\right)=\operatorname{ker}(\wedge \mathrm{e})=0
$$

$\mathrm{e}$

$$
H^{4 m}\left(T_{1} \mathbb{C} P^{2 m}\right) \cong \operatorname{Im}\left(\pi^{*}\right)=0
$$

- Se $2 m \leq k \leq 4 m-1$ temos $2 \leq 2 k+1-(4 m-1) \leq 4 m$. Assim, de

$$
0 \rightarrow H^{2 k+1}\left(T_{1} \mathbb{C} P^{2 m}\right) \stackrel{\pi_{\varkappa}}{\rightarrow} H^{2 k+1-(4 m-1)}\left(\mathbb{C} P^{2 m}\right) \stackrel{\wedge e}{\rightarrow} 0 \stackrel{\pi_{*}^{*}}{\rightarrow} H^{2 k+2}\left(T_{1} \mathbb{C} P^{2 m}\right) \rightarrow H^{2 k+2-(4 m-1)}\left(\mathbb{C} P^{2 m}\right) \rightarrow 0
$$

segue que

$$
H^{2 k+1}\left(T_{1} \mathbb{C} P^{2 m}\right) \cong H^{2 k+1-(4 m-1)}\left(\mathbb{C} P^{2 m}\right)=H^{2(k+1-2 m)}\left(\mathbb{C} P^{2 m}\right) \cong \mathbb{R}
$$

e

$$
H^{2 k+2}\left(T_{1} \mathbb{C} P^{2 m}\right) \cong H^{2 k+2-(4 m-1)}\left(\mathbb{C} P^{2 m}\right)=H^{2(k+1-2 m)+1}\left(\mathbb{C} P^{2 m}\right)=0
$$

Logo,

$$
H^{p}\left(T_{1} \mathbb{C} P^{n}\right) \cong \begin{cases}0, & \text { se pé par e } 4 m+1 \leq p \leq 8 m-1 \\ \mathbb{R}, & \text { se pé ímpar e } 4 m+1 \leq p \leq 8 m-1 .\end{cases}
$$

Tomando a fibração $S^{1} \rightarrow T_{1} \mathbb{C} P^{2 m} \rightarrow \mathbb{C}$ obtemos a sequência de Gysin

$$
\cdots \longrightarrow H^{p}\left(T_{1} \mathbb{C} P^{2 m}\right) \stackrel{\pi_{*}}{\longrightarrow} H^{p-1}(C) \stackrel{\wedge \mathrm{e}}{\longrightarrow} H^{p+1}(C) \stackrel{\pi^{*}}{\longrightarrow} H^{p+1}\left(T_{1} \mathbb{C} P^{2 m}\right) \longrightarrow \cdots
$$

- Se $p=0$ temos

$$
H^{0}\left(T_{1} \mathbb{C} P^{2 m}\right) \rightarrow \underbrace{H^{-1}(C)}_{=0} \rightarrow H^{1}(C) \rightarrow \underbrace{H^{1}\left(T_{1} \mathbb{C} P^{2 m}\right)}_{=0}
$$

Logo, $H^{1}(C)=0$

- Se $p=1$ temos

$$
\underbrace{H^{1}\left(T_{1} \mathbb{C} P^{2 m}\right)}_{=0} \rightarrow H^{0}(C) \stackrel{\wedge e}{\rightarrow} H^{2}(C) \stackrel{\pi^{*}}{\rightarrow} H^{2}\left(T_{1} \mathbb{C} P^{2 m}\right) \rightarrow \underbrace{H^{1}(C)}_{=0} \rightarrow H^{3}(C) \rightarrow \underbrace{H^{3}\left(T_{1} \mathbb{C} P^{2 m}\right)}_{=0}
$$

Pela Observação 3.40 obtemos $j^{*}: H^{2}\left(T_{1} \mathbb{C} P^{2 m}\right) \rightarrow H^{2}(C)$ tal que $j^{*} \circ \pi^{*}=I d$. Logo, pelo Lema de Splitting segue-se que

$$
H^{2}(C) \cong H^{0}(C) \oplus H^{2}\left(T_{1} \mathbb{C} P^{2 m}\right) \cong \mathbb{R} \oplus \mathbb{R} \quad \text { e } \quad H^{3}(C)=0 .
$$

- Para $0 \leq k \leq 2 m-2$ temos

$$
\underbrace{H^{2 k+1}\left(T_{1} \mathbb{C} P^{2 m}\right)}_{=0} \rightarrow H^{2 k}(C) \stackrel{\wedge e}{\rightarrow} H^{2 k+2}(C) \stackrel{\pi^{*}}{\rightarrow} H^{2 k+2}\left(T_{1} \mathbb{C} P^{2 m}\right) \rightarrow \underbrace{H^{2 k+1}(C)}_{=0} \rightarrow H^{2 k+3}(C) \rightarrow \underbrace{H^{2 k+3}\left(T_{1} \mathbb{C} P^{2 m}\right)}_{=0}
$$

Assim, $H^{2 k+3}(C)=0$ e por indução obtemos

$$
H^{2 k+2}(C) \cong H^{2 k}(C) \oplus H^{2 k+2}\left(T_{1} \mathbb{C} P^{2 m}\right) \cong \underbrace{\mathbb{R} \oplus \cdots \oplus \mathbb{R}}_{k+2 \text { vezes }} .
$$


- Se $p=4 m-1$ temos

$$
\underbrace{H^{4 m-1}\left(T_{1} \mathbb{C} P^{2 m}\right)}_{=0} \rightarrow H^{4 m-2}(C) \rightarrow H^{4 m}(C) \rightarrow \underbrace{H^{4 m}\left(T_{1} \mathbb{C} P^{2 m}\right)}_{=0}
$$

Logo,

$$
H^{4 m}(C) \cong H^{4 m-2}(C) \cong \underbrace{\mathbb{R} \oplus \cdots \oplus \mathbb{R}}_{2 m \text { vezes }} .
$$

- Se $p=4 m+1$ temos

$$
0 \rightarrow H^{4 m+1}(C) \stackrel{\pi^{*}}{\rightarrow} \underbrace{H^{4 m+1}\left(T_{1} \mathbb{C} P^{2 m}\right)}_{\cong \mathbb{R}} \stackrel{\pi_{\varkappa}}{\rightarrow} \underbrace{H^{4 m}(C)}_{\substack{\cong \mathbb{R} \oplus \cdots \oplus \mathbb{R} \\ 2 m \text { vezes }}} \stackrel{\wedge \mathrm{e}}{\rightarrow} H^{4 m+2}(C) \rightarrow \underbrace{H^{4 m+2}\left(T_{1} \mathbb{C} P^{2 m}\right)}_{=0}
$$

Como $\pi_{*}$ é injetora temos

$$
H^{4 m+1}(C) \cong \operatorname{Im}\left(\pi^{*}\right)=\operatorname{ker}\left(\pi_{*}\right)=0 .
$$

Como

$$
\operatorname{dim} \operatorname{ker}(\wedge \mathrm{e})=\operatorname{dim} \operatorname{Im}\left(\boldsymbol{\pi}_{*}\right)=\operatorname{dim} H^{4 m+1}\left(T_{1} \mathbb{C} P^{2 m}\right)-\operatorname{dim} \operatorname{ker}\left(\pi_{*}\right)=1
$$

temos

$$
\operatorname{dim} \operatorname{Im}(\wedge \mathrm{e})=\operatorname{dim} H^{4 m}(C)-\operatorname{dim} \operatorname{ker}(\wedge \mathrm{e})=2 m-1 .
$$

Logo,

$$
H^{4 m+2}(C) \cong \operatorname{Im}(\wedge \mathrm{e}) \cong \mathbb{R} \oplus \cdots \oplus \mathbb{R} .
$$

- Por indução, para $2 m+1 \leq k \leq 4 m-1$ temos

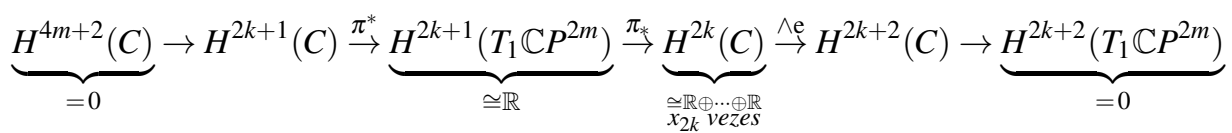

onde $x_{2 k}=\frac{(8 m-2)-2 k}{2}+1=4 m-k$. E pela mesma argumentação do caso anterior obtemos

$$
H^{2 k+1}(C) \cong \operatorname{Im}\left(\pi^{*}\right)=\operatorname{ker}\left(\pi_{*}\right)=0
$$

e

$$
H^{2 k+2}(C) \cong \operatorname{Im}(\wedge \mathrm{e}) \cong \underbrace{\mathbb{R} \oplus \cdots \oplus \mathbb{R}}_{x_{2 k+2}-1 \text { vezes }}
$$

Portanto, $b_{0}(C)=1, b_{2}(C)=2, b_{4}(C)=3, \ldots, b_{4 m-2}(C)=2 m, b_{4 m}(C)=2 m, b_{2 m+2}(C)=2 m-2, \ldots, b_{8 m-2}(C)=1$ e $b_{p}(C)=0$ se pé ímpar.

Lema 3.44. Sejam $M=\mathbb{H} P^{n}$ e $C=T_{1} \mathbb{H} P^{n} / S^{1}$. Então, os números de Betti de $C$ são:

$b_{0}(C)=b_{2}(C)=1, b_{4}(C)=b_{6}(C)=2, \ldots, b_{4 n-4}(C)=b_{4 n-2}(C)=n=b_{4 n}=b_{4 n+2}(C), \ldots, b_{8 n-2}(C)=1$

e $b_{p}=0$ se pé ímpar.

Demonstração. Verificaremos o caso quando $n=4 m$. Os casos quando $n=4 m+1, n=4 m+2, n=4 m+3$ são análogos. Tome a fibração $S^{16 m-1} \rightarrow T_{1} \mathbb{H} P^{4 m} \rightarrow \mathbb{H} P^{4 m}$. A sequência de Gysin associada a esta fibração é:

$$
\cdots \longrightarrow H^{p}\left(T_{1} \mathbb{H} P^{4 m}\right) \stackrel{\pi_{*}}{\longrightarrow} H^{p-(16 m-1)}\left(\mathbb{H} P^{4 m}\right) \stackrel{\wedge \mathrm{e}}{\longrightarrow} H^{p+1}\left(\mathbb{H} P^{4 m}\right) \stackrel{\pi^{*}}{\longrightarrow} H^{p+1}\left(T_{1} \mathbb{H} P^{4 m}\right) \longrightarrow \cdots
$$

Como $T_{1} \mathbb{H} P^{4 m}$ é conexo temos $H^{0}\left(T_{1} \mathbb{H} P^{4 m}\right) \cong \mathbb{R}$. Além disso, sabe-se que

$$
H^{k}\left(\mathbb{H} P^{n}\right)= \begin{cases}\mathbb{R}, & \text { se } k=0,4,8, \ldots \\ 0, & \text { caso contrário }\end{cases}
$$

Disso segue que: 
- Para $1 \leq p \leq 16 m-2$ temos $p-(16 m-1)<0$. Logo, nesses casos $H^{p-(16 m-1)}\left(\mathbb{H} P^{4 m}\right)=0$, e como

$$
\underbrace{H^{(p-1)-(16 m-1)}\left(\mathbb{H} P^{4 m}\right)}_{=0} \rightarrow H^{p}\left(\mathbb{H} P^{4 m}\right) \rightarrow H^{p}\left(T_{1} \mathbb{H} P^{4 m}\right) \rightarrow \underbrace{H^{p-(16 m-1)}\left(\mathbb{H} P^{4 m}\right)}_{=0}
$$

segue-se que $H^{p}\left(T_{1} \mathbb{H} P^{4 m}\right) \cong H^{p}\left(\mathbb{H} P^{4 m}\right)$. Então, se $1 \leq p \leq 16 m-2$ temos

$$
H^{p}\left(T_{1} \mathbb{H} P^{n}\right)= \begin{cases}\mathbb{R}, & \text { se } p=0,4,8, \ldots, 16 m-4 \\ 0, & \text { caso contrário. }\end{cases}
$$

- Se $p=16 m-1$ temos

$$
0 \rightarrow H^{16 m-1}\left(T_{1} \mathbb{H} P^{4 m}\right) \stackrel{\pi_{\hookleftarrow}}{\rightarrow} \underbrace{H^{0}\left(\mathbb{H} P^{4 m}\right)}_{\cong \mathbb{R}} \stackrel{\wedge e}{\rightarrow} \underbrace{H^{16 m}\left(\mathbb{H} P^{4 m}\right)}_{\cong \mathbb{R}} \stackrel{\pi^{*}}{\rightarrow} H^{16 m}\left(T_{1} \mathbb{H} P^{4 m}\right) \rightarrow \underbrace{H^{1}\left(\mathbb{H} P^{4 m}\right)}_{=0}
$$

Como a característica de Euler de $\mathbb{H} P^{n}$ é $n+1$ então pela Proposição 3.37 temos

$$
\int_{\mathbb{H} P^{4 m}} \mathrm{e}=4 m+1 \Longrightarrow \mathrm{e} \neq 0
$$

Assim, $\operatorname{dim} \operatorname{Im}(\wedge \mathrm{e}) \geq 1$ e como $\operatorname{Im}(\wedge \mathrm{e}) \subset H^{16 m}\left(\mathbb{H} P^{4 m}\right)$ temos

$$
1 \leq \operatorname{dim} \operatorname{Im}(\wedge \mathrm{e}) \leq \operatorname{dim} H^{16 m}\left(\mathbb{H} P^{4 m}\right)=1 \Longrightarrow \operatorname{dim} \operatorname{Im}(\wedge \mathrm{e})=1
$$

e disso segue que

- $\operatorname{dim} \operatorname{ker}(\wedge \mathrm{e})=\operatorname{dim} H^{0}\left(\mathbb{H} P^{4 m}\right)-\operatorname{dim} \operatorname{Im}(\wedge \mathrm{e})=1-1=0$

- $\mathbb{R} \cong \operatorname{Im}(\wedge \mathrm{e})=\operatorname{ker}\left(\pi^{*}\right)$

$-\operatorname{dim} \operatorname{Im}\left(\pi^{*}\right)=\operatorname{dim} H^{16 m}\left(\mathbb{H} P^{4 m}\right)-\operatorname{dim} \operatorname{ker}\left(\pi^{*}\right)=0$

Logo,

$$
H^{16 m-1}\left(T_{1} \mathbb{H} P^{4 m}\right) \cong \operatorname{Im}\left(\pi_{*}\right)=\operatorname{ker}(\wedge \mathrm{e})=0
$$

e

$$
H^{16 m}\left(T_{1} \mathbb{H} P^{4 m}\right) \cong \operatorname{Im}\left(\pi^{*}\right)=0 .
$$

- Se $8 m \leq k \leq 16 m-1$ temos $2 \leq p_{k}:=2 k+1-(16 m-1) \leq 16 m$. Além disso,

$$
H^{2 k+1}\left(\mathbb{H} P^{4 m}\right)=H^{2 k+2}\left(\mathbb{H} P^{4 m}\right)=H^{2 k+3}\left(\mathbb{H} P^{4 m}\right)=0
$$

pois $2 k+3>2 k+2>2 k+1>16 m$. Disso segue que

$$
0 \rightarrow H^{2 k+1}\left(T_{1} \mathbb{H} P^{4 m}\right) \rightarrow H^{p_{k}}\left(\mathbb{H} P^{4 m}\right) \rightarrow \underbrace{H^{2 k+2}\left(\mathbb{H} P^{4 m}\right)}_{=0} \rightarrow H^{2 k+2}\left(T_{1} \mathbb{H} P^{4 m}\right) \rightarrow H^{p_{k}+1}\left(\mathbb{H} P^{4 m}\right) \rightarrow 0
$$

Assim,

$$
H^{2 k+2}\left(T_{1} \mathbb{H} P^{4 m}\right) \cong H^{p_{k}+1}\left(\mathbb{H} P^{4 m}\right)=H^{2(k+1-8 m)+1}\left(\mathbb{H} P^{4 m}\right)=0
$$

e

$$
H^{2 k+1}\left(T_{1} \mathbb{H} P^{4 m}\right) \cong H^{p_{k}}\left(\mathbb{H} P^{4 m}\right)= \begin{cases}\mathbb{R}, & \text { se k é ímpar } \\ 0, & \text { se k é par }\end{cases}
$$

pois:

- Se $k$ é ímpar, isto é, $k=2 x+1$, então

$$
p_{k}=2(k+1)-16 m=2(2 x+2)-16 m=4(x+1-4 m) .
$$


- Se $k$ é par, isto é, $k=2 x$ temos

$$
p_{k}=2(k+1)-16 m=2(2 x+1)-16 m=4(x-4 m)+2 .
$$

Logo, para $16 m+1 \leq p \leq 32 m$ temos

$$
H^{p}\left(T_{1} \mathbb{H} P^{4 m}\right)= \begin{cases}\mathbb{R}, & \text { se } p=4 s+3, s \in \mathbb{Z} \\ 0, & \text { caso contrário. }\end{cases}
$$

Tomando a fibração $S^{1} \rightarrow T_{1} \mathbb{H} P^{4 m} \rightarrow C$ obtemos a sequência de Gysin

$\cdots \longrightarrow H^{p}\left(T_{1} \mathbb{H} P^{4 m}\right) \stackrel{\pi_{*}}{\longrightarrow} H^{p-1}(C) \stackrel{\wedge \mathrm{e}}{\longrightarrow} H^{p+1}(C) \stackrel{\pi^{*}}{\longrightarrow} H^{p+1}\left(T_{1} \mathbb{H} P^{4 m}\right) \longrightarrow \cdots$

- Se $p=0$ temos

$$
H^{0}\left(T_{1} \mathbb{H} P^{4 m}\right) \rightarrow \underbrace{H^{-1}(C)}_{=0} \rightarrow H^{1}(C) \rightarrow \underbrace{H^{1}\left(T_{1} \mathbb{H} P^{4 m}\right)}_{=0}
$$

Logo, $H^{1}(C)=0$.

- Se $p=1$ temos

$$
\underbrace{H^{1}\left(T_{1} \mathbb{H} P^{4 m}\right)}_{=0} \rightarrow H^{0}(C) \rightarrow H^{2}(C) \rightarrow \underbrace{H^{2}\left(T_{1} \mathbb{H} P^{4 m}\right)}_{=0} \rightarrow H^{1}(C) \rightarrow H^{3}(C) \rightarrow \underbrace{H^{3}\left(T_{1} \mathbb{H} P^{4 m}\right)}_{=0}
$$

$\operatorname{Logo}, H^{2}(C) \cong H^{0}(C) \cong \mathbb{R}$ e $H^{3}(C) \cong H^{1}(C)=0$.

- Se $p=3$ temos

$$
\underbrace{H^{3}\left(T_{1} \mathbb{H} P^{4 m}\right)}_{=0} \rightarrow H^{2}(C) \rightarrow H^{4}(C) \rightarrow \underbrace{H^{4}\left(T_{1} \mathbb{H} P^{4 m}\right)}_{\cong \mathbb{R}} \rightarrow \underbrace{H^{3}(C)}_{=0} \rightarrow H^{5}(C) \rightarrow \underbrace{H^{5}\left(T_{1} \mathbb{H} P^{4 m}\right)}_{=0}
$$

Pela Observação 3.40 obtemos $j^{*}: H^{4}\left(T_{1} \mathbb{C} P^{2 m}\right) \rightarrow H^{4}(C)$ tal que $j^{*} \circ \pi^{*}=I d$. Logo, pelo Lema de Splitting segue-se que

$$
H^{4}(C) \cong H^{2}(C) \oplus H^{4}\left(T_{1} \mathbb{H} P^{4 m}\right) \cong \mathbb{R} \oplus \mathbb{R} \quad \text { e } \quad H^{5}(C)=0 .
$$

- Para $0 \leq k \leq 4 m-2$ temos

$$
0 \rightarrow H^{4 k}(C) \rightarrow H^{4 k+2}(C) \rightarrow \underbrace{H^{4 k+2}\left(T_{1} \mathbb{H} P^{4 m}\right)}_{=0} \rightarrow H^{4 k+1}(C) \rightarrow H^{4 k+3}(C) \rightarrow 0
$$

$$
0 \rightarrow H^{4 k+2}(C) \rightarrow H^{4 k+4}(C) \rightarrow \underbrace{H^{4 k+4}\left(T_{1} \mathbb{H} P^{4 m}\right)}_{\cong \mathbb{R}} \rightarrow \underbrace{H^{4 k+3}(C)}_{=0} \rightarrow H^{4 k+5}(C) \rightarrow 0
$$

pois $2 \leq 4 k+2<4 k+4 \leq 16 m-4$. Logo, indutivamente obtemos

$$
H^{4 k+2}(C) \cong H^{4 k}(C) \cong \underbrace{\mathbb{R} \oplus \cdots \oplus \mathbb{R}}_{(k+1) \text {-vezes }}, \quad H^{4 k+3}(C) \cong H^{4 k+1}(C)=0
$$

$\mathrm{e}$

$$
H^{4 k+4}(C) \cong H^{4 k+2}(C) \oplus H^{4 k+4}\left(T_{1} \mathbb{H} P^{4 m}\right) \cong \underbrace{\mathbb{R} \oplus \cdots \oplus \mathbb{R}}_{(k+2) \text {-vezes }}, \quad H^{4 k+5}(C)=0 .
$$


- Se $p=16 m-3$ temos

$$
0 \rightarrow H^{16 m-4}(C) \rightarrow H^{16 m-2}(C) \rightarrow \underbrace{H^{16 m-2}\left(T_{1} \mathbb{H} P^{4 m}\right)}_{=0} \rightarrow H^{16 m-3}(C) \rightarrow H^{16 m-1}(C) \rightarrow 0 .
$$

Logo,

$$
H^{16 m-2}(C) \cong H^{16 m-4}(C) \cong \underbrace{\mathbb{R} \oplus \cdots \oplus \mathbb{R}}_{4 m-\text { vezes }} \quad \text { e } \quad H^{16 m-1}(C) \cong H^{16 m-3}(C)=0 .
$$

- Se $p=16 m-1$ temos

$$
0 \rightarrow H^{16 m-2}(C) \rightarrow H^{16 m}(C) \rightarrow \underbrace{H^{16 m}\left(T_{1} \mathbb{H} P^{4 m}\right)}_{=0} \rightarrow H^{16 m-1}(C) \rightarrow H^{16 m+1}(C) \rightarrow 0
$$

Logo,

$$
H^{16 m}(C) \cong H^{16 m-2}(C) \cong \underbrace{\mathbb{R} \oplus \cdots \oplus \mathbb{R}}_{4 m-\text { vezes }} \text { e } \quad H^{16 m+1}(C) \cong H^{16 m-1}(C)=0 .
$$

- Se $p=16 m+1$ temos

$$
\underbrace{H^{16 m+1}\left(T_{1} \mathbb{H} P^{4 m}\right)}_{=0} \rightarrow H^{16 m}(C) \rightarrow H^{16 m+2}(C) \rightarrow \underbrace{H^{16 m+2}\left(T_{1} \mathbb{H} P^{4 m}\right)}_{=0}
$$

Logo,

$$
H^{16 m}(C) \cong H^{16 m-2}(C) \cong \underbrace{\mathbb{R} \oplus \cdots \oplus \mathbb{R}}_{4 m-\text { vezes }}
$$

Continuando a sequência acima temos

$$
0 \rightarrow 0 \rightarrow H^{16 m+3}(C) \stackrel{\pi^{*}}{\rightarrow} \underbrace{H^{16 m+3}\left(T_{1} \mathbb{H} P^{4 m}\right)}_{\cong \mathbb{R}} \stackrel{\pi_{*}}{\rightarrow} \underbrace{H^{16 m+2}(C)}_{\substack{\cong \mathbb{R} \oplus \cdots \oplus \mathbb{R} \\ 4 m-\text { vezes }}} \stackrel{\wedge \mathrm{e}}{\rightarrow} H^{16 m+4}(C) \rightarrow 0
$$

Como $\pi_{*}$ é injetora e $\operatorname{dim} H^{16 m+2}\left(T_{1} \mathbb{H} P^{4 m}\right)=1$ então $\operatorname{dim} \operatorname{Im}\left(\pi_{*}\right)=1$ e $\mathbb{R} \cong \operatorname{Im}\left(\pi_{*}\right)=\operatorname{ker}(\wedge \mathrm{e})$. Assim, $\operatorname{dim} \operatorname{Im}(\wedge \mathrm{e})=\operatorname{dim} H^{16 m+2}(C)-\operatorname{dim} \operatorname{ker}(\wedge \mathrm{e})=4 m-1 \mathrm{e}$, portanto,

$$
H^{16 m+3}(C) \cong \operatorname{Im}\left(\pi^{*}\right)=\operatorname{ker}\left(\pi_{*}\right)=0
$$

$\mathrm{e}$

$$
H^{16 m+4}(C) \cong \operatorname{Im}(\wedge \mathrm{e}) \cong \underbrace{\mathbb{R} \oplus \cdots \oplus \mathbb{R}}_{(4 m-1)-\text { vezes }} .
$$

- Por indução, para $4 m \leq k \leq 8 m-2$ temos

$$
0 \rightarrow H^{4 k+3}(C) \stackrel{\pi^{*}}{\rightarrow} \underbrace{H^{4 k+3}\left(T_{1} \mathbb{H} P^{4 m}\right)}_{\cong \mathbb{R}} \stackrel{\pi_{*}}{\rightarrow} \underbrace{H^{4 k+2}(C)}_{\substack{\cong \mathbb{R} \oplus \cdots \mathbb{R} \\ x_{4 k+2}-\text { vezes }}} \stackrel{\wedge \mathrm{e}}{\rightarrow} H^{4 k+4}(C) \rightarrow 0
$$

onde

$$
x_{4 k+2}:=\frac{(32-4)-\{\text { múltiplo de } 4 \text { menor ou igual a } 4 k+2\}}{4}+1=\frac{32 m-4-4 k}{4}+1=8 m-k .
$$

Pelo mesmo processo do caso anterior obtemos

$$
H^{4 k+3}(C)=0 \text { e } H^{4 k+4}(C) \cong \underbrace{\mathbb{R} \oplus \cdots \oplus \mathbb{R}}_{\left(x_{4 k+2}-1\right)-\text { vezes }}
$$


Seguindo a sequência acima temos

$$
0 \rightarrow H^{4 k+3}(C) \rightarrow H^{4 k+5}(C) \rightarrow \underbrace{H^{4 k+5}\left(T_{1} \mathbb{H} P^{4 m}\right)}_{=0} \rightarrow H^{4 k+4}(C) \rightarrow H^{4 k+6}(C) \rightarrow 0
$$

Logo,

$$
H^{4 k+5}(C) \cong H^{4 k+3}(C)=0 \text { e } H^{4 k+6}(C) \cong H^{4 k+4}(C) \cong \underbrace{\mathbb{R} \oplus \cdots \oplus \mathbb{R}}_{\left(x_{4 k+2}-1\right) \text {-vezes }}
$$

Portanto, temos

$$
\begin{gathered}
b_{0}(C)=b_{2}(C)=1, b_{4}(C)=b_{6}(C)=2, \ldots, b_{16 m-4}(C)=b_{16 m-2}(C)=4 m, \\
b_{16 m}(C)=b_{16 m+2}(C)=4 m, \ldots, b_{32 m-4}(C)=b_{32 m-2}(C)=1 .
\end{gathered}
$$




\section{Capítulo 4}

\section{Variedades Simpléticas e Sistemas Hamiltonianos}

\section{1 Álgebra Linear Simplética}

Definição 4.1. Um espaço vetorial simplético $(V, \omega)$ é um espaço vetorial real $V$ munido de uma forma bilinear $\omega: V \times V \rightarrow \mathbb{R}$ que é

- anti-simétrica: $\omega(u, v)=-\omega(v, u)$ para todo $u, v \in V$.

- não-degenerada: $\omega(u, v)=0$ para todo $v \in V \Longrightarrow u=0$.

A forma $\omega$ é dita uma forma bilinear simplética em V.

Observação 4.2. A forma bilinear $\omega$ é as vezes chamada de uma estrutura simplética linear em V.

Exemplo 4.3. O exemplo mais simples de espaço vetorial simplético é $\mathbb{R}^{2 n}$ equipado com a forma

$$
\omega_{0}=\sum_{i=1}^{n} d x_{i} \wedge d y_{i}
$$

onde $x_{1}, \ldots, x_{n}, y_{1}, \ldots, y_{n}$ são coordenadas em $\mathbb{R}^{2 n}$. Se $\langle$,$\rangle é o produto Euclideano canônico de \mathbb{R}^{2 n}$ então a forma simplética $\omega_{0}$ se escreve como

$$
\omega_{0}(X, Y)=\left\langle J_{0} X, Y\right\rangle
$$

para todo $X, Y \in \mathbb{R}^{2 n}$, onde $J_{0}$ é a matriz em blocos

$$
J_{0}=\left(\begin{array}{cc}
0 & -I d \\
I d & 0
\end{array}\right)
$$

Exemplo 4.4. Seja $V$ um espaço vetorial de dimensão finita e $V^{*}$ o seu dual. Então, $V \oplus V^{*}$ com a forma bilinear

$$
\omega(X+f, Y+g)=f(Y)-g(X)
$$

é um espaço vetorial simplético.

Definição 4.5. Seja $(V, \omega)$ um espaço vetorial simplético de $L \subset V$ um subespaço vetorial. O ortogonal simplético de $L$ é o conjunto

$$
L^{\omega}=\{u \in V ; \omega(u, v)=0 \text { para todo } v \in L\} .
$$

Teorema 4.6. Seja $V$ um espaço vetorial de dimensão finita e $\omega$ uma forma bilinear anti-simétrica em $V$. Então, existe uma base $u_{1}, \ldots, u_{k}, e_{1}, \ldots, e_{n}, f_{1}, \ldots, f_{n}$ de $V$ tal que

- $\omega\left(u_{i}, v\right)=0$, para todo $1 \leq i \leq k$ e para todo $v \in V$, 
- $\omega\left(e_{i}, e_{j}\right)=0=\omega\left(f_{i}, f_{j}\right)$, para todo $1 \leq i, j \leq n e$

- $\omega\left(e_{i}, f_{j}\right)=\delta_{i j}$, para todo $1 \leq i, j \leq n$.

Demonstração. Seja $U=\{u \in V ; \omega(u, v)=0$ para todo $v \in V\}$. Escolha uma base $u_{1}, \ldots, u_{k}$ de $U$ e um espaço complementar $W$ de $U$ em $V$ tal que

$$
V=U \oplus W
$$

Como $U \cap W=\{0\}$ segue que para qualquer $e_{1} \in W$ não nulo existe $f_{1} \in W$ tal que $\omega\left(e_{1}, f_{1}\right) \neq 0$. Então, fixando $e_{1}$ e $f_{1}$ com essa propriedade podemos assumir que $\omega\left(e_{1}, f_{1}\right)=1$. Seja

$$
W_{1}=\operatorname{span}\left\{e_{1}, f_{1}\right\}
$$

Veja que se $v=a e_{1}+b f_{1} \in W_{1} \cap W_{1}^{\omega}$ temos

$$
\begin{aligned}
& 0=\omega\left(v, e_{1}\right)=-b \\
& 0=\omega\left(v, f_{1}\right)=a
\end{aligned}
$$

Logo, $v=0$, ou seja, $W_{1} \cap W_{1}^{\omega}=\{0\}$. Além disso, se $v \in W$ é tal que $\omega\left(v, e_{1}\right)=c$ e $\omega\left(v, f_{1}\right)=d$ segue que

$$
v=\left(-c f_{1}+d e_{1}\right)+\left(v+c f_{1}-d e_{1}\right)
$$

onde $\left(-c f_{1}+d e_{1}\right) \in W_{1}$ e $\left(v+c f_{1}-d e_{1}\right) \in W_{1}^{\omega}$. Portanto, $W=W_{1} \oplus W_{1}^{\omega}$

Tome agora $e_{2} \in W_{1}^{\omega}$ não nulo. Então, existe $f_{2} \in W_{1}^{\omega}$ tal que $\omega\left(e_{2}, f_{2}\right) \neq 0$. Assuma que $\omega\left(e_{2}, f_{2}\right)=1$ e tome $W_{2}=\operatorname{span}\left\{e_{2}, f_{2}\right\}$. Pelo mesmo argumento anterior podemos mostrar que $W_{1}^{\omega}=W_{2} \oplus W_{2}^{\omega}$. Como $\operatorname{dim} V<\infty$ esse processo eventualmente acaba e, assim, obtemos para algum $n \in \mathbb{N}$

$$
V=U \oplus W_{1} \oplus W_{2} \oplus \cdots \oplus W_{n}
$$

onde todos os somandos são ortogonais com respeito a $\omega$ e onde $W_{i}$ tem base $e_{i}, f_{i}$ com $\omega\left(e_{i}, f_{i}\right)=1$.

Corolário 4.7. Seja $(V, \omega)$ um espaço vetorial simplético. Então, $\operatorname{dim} V$ é par e existe uma base $e_{1}, \ldots, e_{n}$, $f_{1}, \ldots, f_{n}$ de $V$ tal que

$$
\omega\left(e_{i}, f_{j}\right)=\delta_{i j} \quad e \quad \omega\left(e_{i}, e_{j}\right)=\omega\left(f_{i}, f_{j}\right)=0 .
$$

Demonstração. Como $\omega$ é não-degenerado segue que

$$
U=\{u \in V ; \omega(u, v)=0 \text { para todo } v \in V\}=\{0\}
$$

Então, pelo Teorema 4.6 segue o desejado.

Definição 4.8. Sejam $\left(V_{1}, \omega_{1}\right)$ e $\left(v_{2}, \omega_{2}\right)$ espaços vetoriais simpléticos. Dizemos que uma transformação linear $T: V_{1} \rightarrow V_{2}$ é simplética se $T^{*} \omega_{2}=\omega_{1}$, ou seja, se $\omega_{2}(T(u), T(v))=\omega_{1}(u, v)$ para quaisquer $u, v \in V_{1}$. Se $V_{1}$ e $V_{2}$ tem a mesma dimensão então dizemos que $T$ é um simplectomorfismo e que $\left(V_{1}, \omega_{1}\right)$ e $\left(V_{2}, \omega_{2}\right)$ são simplectomorfos.

Proposição 4.9. Seja $(V, \omega)$ um espaço vetorial simplético. Então para qualquer subespaço $W \subset V$ vale

$$
\operatorname{dim} V=\operatorname{dim} W+\operatorname{dim} W^{\omega} .
$$

Demonstração. Seja $W^{0}=\left\{f \in V^{*} ; W \subset \operatorname{ker} f\right\}$ o anulador de $W$. Defina a aplicação $T: V \rightarrow V^{*}$ por

$$
T(v)=\omega(v, \cdot) .
$$


Como $\omega$ é não-degenerada segue que $\operatorname{ker} T=\{0\}$. Logo, $T$ é um isomorfismo. Assim, como

$$
\begin{aligned}
T^{-1}\left(W^{0}\right) & =\left\{v \in V ; T(v) \in W^{0}\right\} \\
& =\{v \in V ; \omega(v, u)=0, \forall u \in W\} \\
& =W^{\omega} .
\end{aligned}
$$

e para qualquer subespaço $W$ de qualquer espaço vetorial $V$ temos $\operatorname{dim} W+\operatorname{dim} W^{0}=\operatorname{dim} V$ concluímos que

$$
\operatorname{dim} V=\operatorname{dim} W+\operatorname{dim} T^{-1}\left(W^{0}\right)=\operatorname{dim} W+\operatorname{dim} W^{\omega} .
$$

\subsection{Variedades Simpléticas}

Definição 4.10. Uma forma simplética em uma variedade M é uma 2-forma $\omega$ satisfazendo:

- $\omega$ é fechada, ou seja, $d \omega=0$

- $\omega$ é não-degenerada, ou seja, se para todo $x \in M$ temos $\omega_{x}(u, v)=0$ para todo $v \in T_{x} M$ então $u=0$.

Definição 4.11. Uma variedade simplética é um par $(M, \omega)$ formado por uma variedade $M$ e uma forma simplética $\omega$ em M.

Exemplo 4.12. O exemplo mais simples e mais importante de variedade simplética é o $\mathbb{R}^{2 n}$ com coordenadas $x^{1}, \ldots, x^{n}, y^{1}, \ldots, y^{n}$ e sua forma simplética canônica

$$
\omega_{0}=\sum_{i=1}^{n} d x^{i} \wedge d y^{i}
$$

De modo geral, qualquer espaço vetorial simplético é uma variedade simplética.

Exemplo 4.13. Outro exemplo importante e que será muito utilizado no decorrer desse trabalho é o fibrado cotangente $\pi: T^{*} M \rightarrow M$ de uma variedade $\mathrm{M}$ dotado da 2 -forma $\omega_{\text {can }}$ que definiremos a seguir. Antes disso, defina a 1-forma tautológica $\alpha_{\text {taut }} \in \Omega^{1}\left(T^{*} M\right)$ por

$$
\alpha_{p}(X)=\xi\left(d \pi_{p} X\right)
$$

para todo $p:=(x, \xi) \in T^{*} M$ e para todo $X \in T_{p}\left(T^{*} M\right)$. Então, defina a 2-forma canônica em $T^{*} M$ por

$$
\omega_{\text {can }}=-d \alpha_{\text {taut }} .
$$

Seja $U \subset M$ um aberto com coordenadas $x^{1}, \ldots, x^{n}$. Então, as 1-formas $d x^{i} \in \Omega^{1}(U)$ associadas são tais que para cada $x \in U,\left.d x^{1}\right|_{x}, \ldots,\left.d x^{n}\right|_{x}$ formam uma base de $T_{x}^{*} M$. Se $\xi \in T_{x}^{*} M$ então $\xi=\sum \xi_{i} d x^{i}$, onde $\xi_{i} \in \mathbb{R}$ são unicamente determinadas por $\xi$. Logo,

$$
x^{1}, \ldots, x^{n}, \xi_{1}, \ldots, \xi_{n}
$$

são coordenadas naturais de $T^{*} U$. Estas coordenadas, por sua vez, induzem coordenadas em $T\left(T^{*} U\right)$ dadas por

$$
\dot{x}^{1}, \ldots, \dot{x}^{n}, \dot{\xi}_{1}, \ldots, \dot{\xi}_{n}
$$

isto é, se $p=(x, \xi)$ e $X_{p} \in T_{p}\left(T^{*} U\right)$ então

$$
X_{p}=\sum_{i=1}^{n} \dot{x}^{i} \frac{\partial}{\partial x^{i}}+\sum_{i=1}^{n} \dot{\xi}_{i} \frac{\partial}{\partial \xi_{i}}
$$


Logo, a 1 -forma $\alpha_{\text {taut }}$ se escreve localmente como

$$
\begin{aligned}
\alpha_{p}\left(X_{p}\right) & =\xi\left(d \pi_{p} X_{p}\right) \\
& =\left(\sum_{i=1}^{n} \xi_{i} d x^{i}\right)\left(\sum_{j=1}^{n} \dot{x}^{j} \frac{\partial}{\partial x^{j}}\right) \\
& =\sum_{i, j=1}^{n} \xi_{i} \dot{x}^{j} d x^{i}\left(\frac{\partial}{\partial x^{j}}\right) \\
& =\sum_{i=1}^{n} \xi_{i} \dot{x}^{i} \\
& =\sum_{i=1}^{n} \xi_{i} d x^{i}
\end{aligned}
$$

Portanto, localmente temos

$$
\omega_{\mathrm{can}}=-d \alpha_{\mathrm{taut}}=\sum_{i=1}^{n} d x^{i} \wedge d \xi_{i}
$$

Definição 4.14. Uma aplicação simplética $\varphi:\left(M_{1}, \omega_{1}\right) \rightarrow\left(M_{2}, \omega_{2}\right)$ entre variedades simpléticas é uma aplicação diferenciável satisfazendo $\varphi^{*} \omega_{2}=\omega_{1}$. Se $\varphi$ for um difeomorfismo, então, dizemos que $\varphi$ é um simplectomorfismo.

Teorema 4.15 (Darboux). Seja $(M, \omega)$ uma variedade simplética de dimensão $2 n$ e seja $p \in M$. Considere $\mathbb{R}^{2 n}$ munido da forma simplética canônica $\omega_{0}$. Então, existem vizinhanças $U \subset M$ de $p, V \subset \mathbb{R}^{2 n}$ de $0 \in \mathbb{R}^{2 n}$ e um simplectomorfismo $\varphi: U \rightarrow V$ tal que $\varphi(p)=0$ e $\varphi^{*} \omega_{0}=\omega$.

Esse teorema nos diz que todas as variedades simpléticas de mesma dimensão são localmente iguais.

\subsection{Equações de Hamilton}

Definição 4.16. Um sistema Hamiltoniano é uma tripla $(M, \omega, H)$ onde $(M, H)$ é uma variedade simplética e $H: M \rightarrow \mathbb{R}$ é uma função suave, chamada de função Hamiltoniana.

Associada a $(M, \omega, H)$ está o campo Hamiltoniano $X_{H}$, definido implicitamente por

$$
i_{X_{H}} \omega=d H, \text { i.e, } d H(y)=\omega\left(X_{H}, y\right) \text { para todo } y \in T M .
$$

Teorema 4.17. Considere $\mathbb{R}^{2 n}$ com coordenadas $\left(p_{1}, \ldots, p_{n}, q_{1}, \ldots, q_{n}\right)$ e $\omega_{0}=\sum_{i=1}^{n} d p_{j} \wedge d q_{j}$. A curva $\rho_{t}=(p(t), q(t))$ é uma curva integral de $X_{H}$ se, e somente se,

$$
\left\{\begin{aligned}
\frac{d p_{i}}{d t}(t) & =\frac{\partial H}{\partial q_{i}}(p(t), q(t)) \\
\frac{d q_{i}}{d t}(t) & =-\frac{\partial H}{\partial p_{i}}(p(t), q(t))
\end{aligned} \quad\right. \text { (Equações de Hamilton) }
$$


Demonstração. Seja $X_{H}=\sum\left(\frac{\partial H}{\partial q_{i}} \frac{\partial}{\partial p_{i}}-\frac{\partial H}{\partial p_{i}} \frac{\partial}{\partial q_{i}}\right)$. Então,

$$
\begin{aligned}
i_{X_{H}} \omega & =\sum_{j=1}^{n} i_{X_{H}}\left(d p_{j} \wedge d q_{j}\right) \\
& =\sum_{j=1}^{n}\left[\left(i_{X_{H}} d p_{j}\right) \wedge d q_{j}-d p_{j} \wedge\left(i_{X_{H}} d q_{j}\right)\right] \\
& =\sum_{j=1}^{n}\left[\frac{\partial H}{\partial q_{j}} d q_{j}+\frac{\partial H}{\partial p_{j}} d p_{j}\right] \\
& =d H
\end{aligned}
$$

Agora sejam

$$
X_{H}=\sum_{i=1}^{n}\left(a_{i} \frac{\partial}{\partial p_{i}}+b_{i} \frac{\partial}{\partial q_{i}}\right) \text { e } d H=\sum_{j=1}^{n}\left(\frac{\partial H}{\partial q_{j}} d q_{j}+\frac{\partial H}{\partial p_{j}} d p_{j}\right)
$$

Então,

$$
d H=i_{X_{H}} \omega=\sum_{i=1}^{n} i_{X_{H}}\left(d p_{i} \wedge d q_{i}\right)=\sum_{i=1}^{n}\left(a_{i} d q_{i}-b_{i} d p_{i}\right) .
$$

Logo,

$$
a_{i}=\frac{\partial H}{\partial q_{i}} \text { e } b_{i}=-\frac{\partial H}{\partial p_{i}}
$$

e como $\rho_{t}$ é uma curva integral de $X_{H}$ segue que $\rho^{\prime}(t)=X_{H}(\rho(t))$, ou seja,

$$
\left(\frac{d p_{1}}{d t}(t), \ldots, \frac{d p_{n}}{d t}(t), \frac{d q_{1}}{d t}(t), \ldots, \frac{d q_{n}}{d t}(t)\right)=\left(a_{1}, \ldots, a_{n}, b_{1}, \ldots, b_{n}\right) .
$$

Logo,

$$
\frac{d p_{i}}{d t}(t)=a_{i} \quad \text { e } \quad \frac{d q_{i}}{d t}(t)=b_{i}, \text { para todo } 1 \leq i \leq n
$$

Portanto,

$$
\left\{\begin{array}{l}
\frac{d p_{i}}{d t}(t)=\frac{\partial H}{\partial q_{i}} \\
\frac{d q_{i}}{d t}(t)=-\frac{\partial H}{\partial p_{i}}
\end{array}\right.
$$

Proposição 4.18 (Conservação de Energia). Seja $(M, \omega)$ uma variedade simplética e $H: M \rightarrow \mathbb{R}$. Então, $H\left(\varphi^{t}(x)\right)=H(x)$ para todo $x \in M$ e $t \in \mathbb{R}$, onde $\varphi^{t}$ é o fluxo de $X_{H}$, ou seja, a função hamiltoniana é constante ao longo das órbitas do seu campo de vetores Hamiltoniano.

Demonstração. Se $\varphi^{t}$ é o fluxo de $X_{H}$ temos

$$
\begin{aligned}
\frac{d}{d t} H\left(\varphi^{t}(x)\right) & =d H\left(\varphi^{t}(x)\right) \frac{d}{d t} \varphi^{t}(x) \\
& =d H\left(\varphi^{t}(x)\right) X_{H}\left(\varphi^{t}(x)\right) \\
& =\omega\left(X_{H}\left(\varphi^{t}(x)\right), X_{H}\left(\varphi^{t}(x)\right)\right) \\
& =0 .
\end{aligned}
$$

Portanto,

$$
H\left(\varphi^{t}(x)\right)=H\left(\varphi^{0}(x)\right)=H(x) \quad \forall t \in \mathbb{R} .
$$




\subsection{O colchete de Poisson}

Definição 4.19. Uma álgebra de Lie é um espaço vetorial $L$ sobre um corpo F juntamente com uma operação binária [,] $: L \times L \rightarrow L$ que satisfaz:

(i) Bilinearidade

$$
[a x+b y, z]=a[x, y]+b[y, z] \text { e }[z, a x+b y]=a[z, x]+b[z, y]
$$

para todos $a, b \in F$ e $x, y, z \in L$.

(ii) Anticomutatividade

$$
[x, y]=-[y, x] \text { para todos } x, y \in L
$$

(iii) A identidade de Jacobi

$$
[x,[y, z]]+[y,[z, x]]+[z,[x, y]]=0
$$

para todos $x, y, z \in L$.

O operador [, ] é chamado de comutador.

Seja $\mathrm{M}$ uma varidade suave e $X: M \rightarrow T M$ um campo de vetores em M. Com cada campo de vetores associamos:

1. O grupo a um parâmetro de difeomorfismos ou fluxo $\phi_{t}: M \rightarrow M$ onde

$$
\left.\frac{d}{d t}\right|_{t=0} \phi_{t}(p)=X(p) .
$$

2. A derivada de Lie. Para qualquer função $f: M \rightarrow \mathbb{R}$ a derivada na direção de $X$ é uma nova função

$$
\left(\mathcal{L}_{X} f\right)(p)=\left.\frac{d}{d t}\right|_{t=0} f\left(\phi_{t}(p)\right)
$$

Definição 4.20. O colchete de Poisson ou comutador de dois campos de vetores $X$ e $Y$ em uma variedade M é o campo de vetor $Z$ para o qual

$$
\mathcal{L}_{Z}=\mathcal{L}_{Y} \mathcal{L}_{X}-\mathcal{L}_{X} \mathcal{L}_{Y} .
$$

O colchete de Poisson de dois campos de vetores será denotado por

$$
Z=\{X, Y\} .
$$

Observação 4.21. Para ver que os operadores da definição acima estão bem definidos veja [Arn13], páginas 209-211.

Suponha que nos são dados dois campos de vetores $X$ e $Y$ em uma variedade M. Os fluxos correspondentes $\phi_{t}$ e $\psi_{t}$ não comutam em geral: $\phi_{t} \psi_{t} \neq \psi_{t} \phi_{t}$. Mas o teorema a seguir nos dá uma condição para que isso ocorra.

Teorema 4.22 ([Arn13], pág 211-212). Os fluxos $\phi_{t}$ e $\psi_{t}$ comutam se, e somente se, o colchete de Poisson dos campos de vetores correspondentes é igual a zero, isto é,

$$
\{X, Y\}=0 .
$$

Seja $(M, \omega)$ uma variedade simplética. Para cada função $H: M \rightarrow \mathbb{R}$ corresponde um grupo a um parâmetro $\psi_{t}^{H}: M \rightarrow M$, onde

$$
\left.\frac{d}{d t}\right|_{t=0} \psi_{t}^{H}(p)=X_{H}(p)
$$

Seja $F: M \rightarrow \mathbb{R}$ outra função em $\mathrm{M}$. 
Definição 4.23. O colchete de Poisson $(F, H)$ das funções $\mathrm{F}$ e $\mathrm{H}$ dadas em uma variedade simplética $(M, \omega)$ é a derivada da função $\mathrm{F}$ na direção do fluxo com função hamiltoniana $\mathrm{H}$, isto é,

$$
(F, H)(p)=\left.\frac{d}{d t}\right|_{t=0} F\left(\psi_{t}^{H}(p)\right) .
$$

Teorema 4.24. Sejam $X_{B}$ e $X_{C}$ campos hamiltonianos com funções hamiltonianas $B$ e $C$. Considere o colchete de Poisson $\left\{X_{B}, X_{C}\right\}$ desses campos de vetores. Então, o campo de vetor $\left\{X_{B}, X_{C}\right\}$ é hamiltoniano e sua função hamiltoniana é igual ao colchete de Poisson das funções hamiltonianas $(B, C)$.

Demonstração. Seja $(B, C)=D$. A identidade de Jacobi pode ser reescrita na forma

$$
(A, D)=((A, B), C)-((A, C), B) .
$$

Então,

$$
\begin{aligned}
\mathcal{L}_{X_{D}} & =\mathcal{L}_{X_{C}} \mathcal{L}_{X_{B}}-\mathcal{L}_{X_{B}} \mathcal{L}_{X_{C}} \\
& =\mathcal{L}_{\left\{X_{B}, X_{C}\right\}}
\end{aligned}
$$

Logo,

$$
X_{D}=\left\{X_{B}, X_{C}\right\}
$$

Teorema 4.25. Os fluxos das funções hamiltonianas $H_{1}$ e $H_{2}$ comutam se, e somente se, o colchete de Poisson das funções $\mathrm{H}_{1}$ e $\mathrm{H}_{2}$ é (localmente) constante.

Demonstração. Pelo Teorema (4.22) uma condição necessário e suficiente para que os fluxos de $H_{1}$ e $H_{2}$ comutem é $\left\{X_{H_{1}}, X_{H_{2}}\right\} \equiv 0$. Pelo Teorema (4.24) temos $X_{\left(H_{1}, H_{2}\right)}=\left\{X_{H_{1}}, X_{H_{2}}\right\}$. Se os fluxos de $H_{1}$ e $H_{2}$ comutam temos

$$
d\left(H_{1}, H_{2}\right)=\omega\left(X_{\left(H_{1}, H_{2}\right)}, \cdot\right)=\omega\left(\left\{X_{H_{1}}, X_{H_{2}}\right\}, \cdot\right)=\omega(0, \cdot) \equiv 0 .
$$

Portanto, $\left(H_{1}, H_{2}\right)$ é (localmente) constante. Agora, se $\left(H_{1}, H_{2}\right)$ é (localmente) constante, temos

$$
0=d\left(H_{1}, H_{2}\right)=\omega\left(X_{\left(H_{1}, H_{2}\right)}, \cdot\right)=\omega\left(\left\{X_{H_{1}}, X_{H_{2}}\right\}, \cdot\right)
$$

Como $\omega$ é não degenerada temos $\left\{X_{H_{1}}, X_{H_{2}}\right\} \equiv 0$ e, portanto, os fluxos de $H_{1}$ e $H_{2}$ comutam.

\subsection{Fluxo Hamiltoniano no Fibrado Cotangente}

Seja $\left\{\phi_{t}\right\}$ um grupo a um paramêtro de difeomorfismos, onde $\phi_{t}: M \rightarrow M$. Defina $H_{1}: T^{*} M \rightarrow \mathbb{R}$ por

$$
H_{1}(p, \xi)=\xi(V(p)) .
$$

onde $V: M \rightarrow T M$ um campo de vetores gerado por $\phi_{t}$.

Teorema 4.26. Seja $\omega=d p \wedge d \xi$ a forma canônica simplética de $T^{*} M$. Então, o fluxo $\psi_{t}^{H_{1}}$ associada a função Hamiltoniana $H_{1}$ é tal que

$$
\psi_{t}^{H_{1}}(p, \xi)=\left(\phi_{t}(p),\left(\left(D \phi_{t}(p)\right)^{-1}\right)^{*} \xi\right) .
$$

Demonstração. Como V é um campo de vetores gerado por $\phi_{t}$ temos

$$
V(p)=\lim _{t \rightarrow 0} \frac{\phi_{t}(p)-p}{t} \quad \text { para todo } p \in M .
$$

Por outro lado, podemos escrever $V(p)=v^{1}(p) \partial p^{1}+\ldots+v^{n}(p) \partial p^{n}$ e $\xi=\xi_{1} d p^{1}+\ldots+\xi_{n} d p^{n}, \xi \in T_{p}^{*} M$. Então,

$$
H_{1}(p, \xi)=\xi(V(p))=\xi_{1} v^{1}(p)+\ldots+\xi_{n} v^{n}(p)
$$


implicando que

$$
\frac{\partial H_{1}}{\partial \xi_{i}}=v^{i}(p) \quad \text { e } \quad \frac{\partial H_{1}}{\partial p_{i}}=\sum_{j=1}^{n} \xi_{j} \frac{\partial v^{j}}{\partial p_{i}}(p) .
$$

Como $\phi_{t+s}=\phi(t+s, p)=\phi(t, \phi(s, p))$ temos

$$
\frac{\partial}{\partial s} \phi(t+s, p)=D \phi(t, \phi(s, p)) \cdot \frac{\partial \phi}{\partial s}(s, p),
$$

onde $D \phi(t, p)$ denota a derivada de $\phi$ em relação a p. Defina $\psi_{t}: T M \rightarrow T M$ por

$$
\psi_{t}(p, u)=\left(\phi_{t}(p), D \phi_{t}(p) u\right)
$$

então, o campo de vetores gerado por esse fluxo é:

$$
\begin{aligned}
Y(p, u) & =\lim _{t \rightarrow 0} \frac{\psi_{t}(p, u)-\psi_{0}(p, u)}{t} \\
& =\lim _{t \rightarrow 0} \frac{\left(\phi_{t}(p), D \phi_{t}(p) u\right)-(p, u)}{t} \\
& =\lim _{t \rightarrow 0}\left(\frac{\phi_{t}(p)-p}{t}, \frac{D \phi_{t}(p) u-u}{t}\right) \\
& =\left(V(p), \lim _{t \rightarrow 0} \frac{D \phi_{t}(p) u-u}{t}\right)
\end{aligned}
$$

Como

$$
\begin{aligned}
\lim _{t \rightarrow 0} \frac{D \phi_{t}(p) u-u}{t} & =\lim _{t \rightarrow 0} D\left(\frac{\phi_{t}(p)-I}{t}\right) u \\
& =D\left(\lim _{t \rightarrow 0} \frac{\phi_{t}(p)-\phi_{0}(p)}{t}\right) u \\
& =D V(p) u
\end{aligned}
$$

temos,

$$
Y(p, u)=(V(p), D V(p) u) \in T T M .
$$

Agora queremos definir um fluxo no fibrado cotangente a partir do fluxo que definimos em (4.8). Seja $\widetilde{\psi}_{t}: T^{*} M \rightarrow T^{*} M$ definido por

$$
\widetilde{\psi}_{t}(p, \xi)=\left(\phi_{t}(p), \xi \circ\left(D \phi_{t}(p)\right)^{-1}\right),
$$

onde $\left(D \phi_{t}(p)\right)^{-1}: T_{\phi_{t}(p)} M \rightarrow T_{p} M$. Como $\left(D \phi_{t}(p)\right)^{-1} \cdot D \phi_{t}(p)=$ Id segue que

$$
\frac{\partial}{\partial t}\left(D \phi_{t}(p)\right)^{-1} \cdot D \phi_{t}(p)+\left(D \phi_{t}(p)\right)^{-1} \cdot \frac{\partial}{\partial t}\left(D \phi_{t}(p)\right)=0
$$

e isto implica que

$$
\begin{aligned}
\left.\frac{\partial}{\partial t}\left(D \phi_{t}(p)\right)^{-1}\right|_{t=0} & =-\left.\left(D \phi_{0}(p)\right)^{-1} \cdot \frac{\partial}{\partial t}\left(D \phi_{t}\right)\right|_{t=0} \cdot\left(D \phi_{0}(p)\right)^{-1} \\
& =-\mathrm{Id} \cdot D V(p) \cdot \mathrm{Id} \\
& =-D V(p)
\end{aligned}
$$


Assim, o campo gerado pelo fluxo $\widetilde{\psi}$ em $T^{*} M$ é:

$$
\begin{aligned}
X(p, \xi) & :=\lim _{t \rightarrow 0} \frac{\widetilde{\psi}_{t}(p, \xi)-\widetilde{\psi}_{0}(p, \xi)}{t} \\
& =\lim _{t \rightarrow 0} \frac{\left(\phi_{t}(p), \xi \circ\left(D \phi_{t}(p)\right)^{-1}\right)-\left(\phi_{0}(p), \xi \circ\left(D \phi_{0}(p)\right)^{-1}\right)}{t} \\
& =\lim _{t \rightarrow 0}\left(\frac{\phi_{t}(p)-p}{t}, \frac{\xi \circ\left(D \phi_{t}(p)\right)^{-1}-\xi \circ\left(D \phi_{0}(p)\right)^{-1}}{t}\right) \\
& =\left(V(p), \xi\left(\left.\frac{\partial}{\partial t}\left(D \phi_{t}(p)\right)^{-1}\right|_{t=0}\right)\right) \\
& =(V(p), \xi(-D V(p)(\cdot))) \\
& =(V(p),-\xi(D V(p)(\cdot)))
\end{aligned}
$$

Como,

$$
-\xi \circ D V_{p}\left(\partial p^{i}\right)=-\sum_{j=1}^{n} \xi^{j} \frac{\partial v^{j}}{\partial p^{i}}(p)=-\frac{\partial H_{1}}{\partial p^{i}}(p, \xi) \text { e } \quad v^{i}(p)=\frac{\partial H_{1}}{\partial \xi^{i}}(p, \xi)
$$

então, pelo Teorema 4.17 segue que

$$
X=X_{H_{1}} \quad \text { e } \quad \psi_{t}^{H_{1}}(p, \xi)=\widetilde{\psi}_{t}(p, \xi)=\left(\phi_{t}(p),\left(\left(D \phi_{t}(p)\right)^{-1}\right)^{*} \xi\right) .
$$

Sejam $(M, g)$ uma variedade Riemanniana e $H_{0}: T^{*} M \rightarrow \mathbb{R}$ uma função hamiltoniana definida por

$$
H_{0}(p, \xi)=\sup \left\{\xi(v) ; v \in T_{p} M \text { e } g(v, v)=1\right\},
$$

isto é, $H_{0}$ é a norma dual de g. Tome $H_{1}$ como definido na equação (4.5), então:

Teorema 4.27. Se $\phi_{t}$ é um grupo a um parâmetro de isometrias, então, os fluxos $\psi_{t}^{H_{0}}$ e $\psi_{t}^{H_{1}}$ associados, respectivamente, as funções hamiltonianas $H_{0}$ e $H_{1}$ comutam.

Demonstração. Como $\phi_{t}$ são isometrias em $\mathrm{M}$ temos

$$
\phi_{t}^{*} g=g \Longrightarrow g(u, v)=g\left(D \phi_{t}(p)(u), D \phi_{t}(p)(v)\right), \text { para tado } u, v \in T_{p} M .
$$

Quero verificar que $\psi_{t}^{H_{1}}$ são isometrias em $T^{*} M$. De fato, pelo Teorema (4.26) temos

$$
\begin{aligned}
\psi_{t}^{H_{1}{ }^{*}} H_{0}(p, \xi) & =H_{0}\left(\psi_{t}^{H_{1}}(p, \xi)\right) \\
& =H_{0}\left(D \phi_{t}(p)^{\left.-1^{*} \xi\right)}\right. \\
& =H_{0}\left(\xi \circ D \phi_{t}(p)^{-1}\right) \\
& =\sup \left\{\xi \circ D \phi_{t}(p)^{-1}(v) ; v \in T_{p} M \text { e } g(v, v)=1\right\} \\
& =\sup \left\{\xi\left(D \phi_{t}(p)^{-1}(v)\right) ; v \in T_{p} M \text { e } g\left(D \phi_{t}(p)^{-1}(v), D \phi_{t}(p)^{-1}(v)\right)=1\right\} \\
& =H_{0}(p, \xi)
\end{aligned}
$$

onde a última igualdade segue do fato de $D \phi_{t}(p)^{-1}$ ser sobrejetora. Então,

$$
\begin{aligned}
\left(H_{0}, H_{1}\right) & =\left.\frac{d}{d t}\right|_{t=0} H_{0}\left(\psi_{t}^{H_{1}}(p, \xi)\right) \\
& =\left.\frac{d}{d t}\right|_{t=0} H_{0}(p, \xi) \\
& =0 .
\end{aligned}
$$


Portanto, pelo Teorema 4.25 os fluxos $\psi_{t}^{H_{0}}$ e $\psi_{t}^{H_{1}}$ comutam.

Teorema 4.28. Sejam $(M, F)$ uma variedade Finsler e $H: T^{*} M \rightarrow \mathbb{R}$ um hamiltoniano tal que $F=H \circ$ $\mathscr{L}_{\frac{1}{2} H^{2}}^{-1}$. Se $X_{\frac{1}{2} H^{2}}$ é o campo de vetores hamiltoniano associado a função $\frac{1}{2} H^{2}$ então $X_{\frac{1}{2} H^{2}}$ descreve as geodésicas da métrica Finsler $F$, isto é, a projeção da curva integral de $X_{\frac{1}{2} H^{2}}$ sobre $\pi: T^{*} M \rightarrow M$ são as geodésicas de F.

Demonstração. Seja

$$
\widetilde{H}(x, \xi)=\frac{1}{2} g^{i j}(\xi) \xi_{i} \xi_{j},
$$

onde $g^{i j}(\xi)$ é a inversa de $g_{i j}$ e $g_{i j}(y) y^{j}=\xi_{i}$ (ver seção 1.2.2). Pelas Proposição 1.16 e Proposição 1.17 obtemos

$$
\begin{aligned}
h^{i j}(\xi) & =\frac{1}{2} \frac{\partial^{2} H^{2}(\xi)}{\partial \xi_{i} \partial \xi_{j}} \\
& =\frac{1}{2} \frac{\partial^{2} F^{2}\left(\mathscr{L}_{\frac{1}{2} F^{2}}^{-1}(\xi)\right)}{\partial \xi_{i} \partial \xi_{j}} \\
& =\frac{1}{2} \frac{\partial^{2} F^{* 2}(\xi)}{\partial \xi_{i} \partial \xi_{j}} \\
& =\left(g^{*}\right)^{i j}(\xi) .
\end{aligned}
$$

Logo, pelas observações feitas na seção 1.2.2 obtemos

$$
\widetilde{H}(x, \xi)=\frac{1}{2} h^{i j}(\xi) \xi_{i} \xi_{j}=\frac{1}{2} H^{2}(x, \xi) .
$$

Pelo Teorema 1.33 o fluxo cogeodésico de F é o fluxo $\psi_{t}^{\tilde{H}}$ associado ao campo Hamiltoniano $X_{\tilde{H}}=X_{\frac{1}{2}} H^{2}$ concluímos que

$$
\pi\left(\psi_{t}^{\tilde{H}}(x, \xi)\right)=\gamma(t), \quad \xi \in T_{x}^{*} M
$$

é uma geodésicas de $F$.

Teorema 4.29. Sejam $(M, F)$ uma variedade Finsler e $F^{*}$ a norma dual de $F$. Se $X_{\frac{1}{2} F^{* 2}}$ é o campo de vetores hamiltoniano associado a função $\frac{1}{2} F^{* 2}$ então $X_{\frac{1}{2}} F^{* 2}$ descreve as geodésicas da métrica Finsler $F$, isto é, a projeção da curva integral de $X_{\frac{1}{2} F^{* 2}}$ sobre $\pi: T^{*} M \rightarrow M$ são as geodésicas de $F$.

Demonstração. Como a função hamiltoniana $H$ definida em (1.24) é, pela igualdade (1.20), tal que

$$
H(x, \xi)=\frac{1}{2} F^{* 2}(x, \xi) .
$$

Então pelo Teorema 1.33 segue o desejado.

\subsection{Bifurcações e o Princípio de Hamilton}

Os resultados dessa seção foram retirados quase exclusivamente de [Wei78] e serão de fundamental importância para as discussões feitas no Capítulo 5.

\subsubsection{Princípio de Hamilton}

Definição 4.30. O espaço de free loop $\Lambda M$ de qualquer variedade $M$ de dimensão finita é o espaço de todas as aplicações $C^{\infty}$ de $S^{1}=\mathbb{R} / \mathbb{Z}$ em M, isto é,

$$
\Lambda M=\left\{c: S^{1} \rightarrow M ; c \text { é } C^{\infty}\right\} .
$$


Definição 4.31. Um caminho suave de $[0,1]$ em $\Lambda M$ é uma família $\left\{c_{s} ; s \in[0,1]\right\}$ de loops para os quais o cilindro $S^{1} \times[0,1] \rightarrow M$ definido por $C(t, s)=c_{s}(t)$ é $C^{\infty}$.

O espaço tangente $T_{c}(\Lambda M)$ em um ponto $c: S^{1} \rightarrow M$ consiste de todos os campos de vetores suaves ao longo de $c$, isto é,

$$
T_{c}(\Lambda M)=\left\{v: S^{1} \rightarrow T M ; \pi \circ v=c\right\} .
$$

onde $\pi: T M \rightarrow M$ é a projeção canônica. De forma equivalente, podemos considerar $T_{c}(\Lambda M)$ como as seções $C^{\infty}$ de $c^{*} T M$ sobre $S^{1}$, onde

$$
c^{*} T M=\left\{(t, x) \in S^{1} \times T M ; c(t)=\pi(x)\right\} .
$$

Notação 4.32. Também denotaremos o espaço tangente por $\Gamma^{\infty}\left(c^{*} T M\right)$.

Notação 4.33. Nessa seção, sendo $\mu: X \rightarrow Y$ uma aplicação, definimos

$$
\langle\mu, x\rangle=\mu(x), \forall x \in X .
$$

Cada 1-forma $\theta \in \Gamma^{\infty}\left(T_{c}^{*} \Lambda M\right)$ ao longo de $c$ define um funcional linear em $T_{c}(\Lambda M)$ pela regra

$$
\langle\theta, v\rangle=\int_{0}^{1}\langle\theta(t), v(t)\rangle d t .
$$

O espaço cotangente $T_{c}^{*}(\Lambda M)$ consiste de todas as distribuições em $S^{1}$ com valores em $c^{*}\left(T^{*} M\right)$. E denotaremos esse espaço por $\Gamma^{-\infty}\left(c^{*} T M\right)$. Se $\alpha$ é uma 1-forma em M então podemos definir uma 1-forma $\Lambda \alpha$ em $\Lambda M$ da seguinte forma:

$$
(\Lambda \alpha)(c)=\alpha \circ c
$$

onde $\alpha(c(t))=\alpha_{c(t)}: T_{c(t)} M \rightarrow \mathbb{R}$. Esta 1-forma em $\Lambda M$ opera em vetores $v \in T_{c}(\Lambda M)$ de acordo com a fórmula

$$
\langle\Lambda \alpha, v\rangle=\int_{0}^{1}\langle\alpha(c(t)), v(t)\rangle d t
$$

Analogamente podemos levantar qualquer k-forma $\beta$ em $\mathrm{M}$ em uma k-forma $\Lambda \beta$ em $\Lambda M$ definindo

$$
\left\langle\Lambda \beta,\left(v_{1}, \cdots, v_{k}\right)\right\rangle=\int_{0}^{1}\left\langle\beta(c(t)),\left(v_{1}(t), \cdots, v_{k}(t)\right)\right\rangle d t .
$$

para todas k-uplas $\left(v_{1}, \cdots, v_{k}\right)$ de campos de vetores suaves ao longo de $c$, isto é, $v_{i} \in T_{c}(\Lambda M)$ para todo $1 \leq i \leq k$.

Lema 4.34. $\Lambda d \alpha=d \Lambda \alpha$ para toda k-forma $\alpha$ em $M$.

Demonstração.

$$
\langle\Lambda d \alpha, \cdot\rangle=\int_{0}^{1}\langle d \alpha(t), \cdot\rangle d t=d \int_{0}^{1} \alpha(t) d t=d(\Lambda \alpha)(\cdot)
$$

Se $\Omega$ é uma 2-forma simplética em $M$ então $\widetilde{\Omega}: T M \rightarrow T^{*} M$ definida por $\widetilde{\Omega}(x)(y)=\Omega(x, y)$ é um isomorfismo. Assim, também podemos associar a $\Lambda \Omega$ em $\Lambda M$ a aplicação $\widetilde{\Lambda \Omega}: T(\Lambda M) \rightarrow T^{*}(\Lambda M)$ definida por

$$
\widetilde{\Lambda \Omega}(v)=\widetilde{\Omega} \circ v .
$$

Lema 4.35. A função $\widetilde{\Lambda \Omega}: T(\Lambda M) \rightarrow T^{*}(\Lambda M)$ é um isomorfismo de cada espaço tangente $T_{c}(\Lambda M)=$ $\Gamma^{\infty}\left(c^{*} T M\right)$ no subespaço $\Gamma^{\infty}\left(c^{*} T^{*} M\right) \subset T_{c}^{*}(\Lambda M)$.

Demonstração. Sejam $v_{1}, v_{2} \in T_{c}(\Lambda M)$ e suponha que $\widetilde{\Lambda \Omega}\left(v_{1}\right)=\widetilde{\Lambda \Omega}\left(v_{2}\right)$. Então,

$$
\widetilde{\Omega} \circ v_{1}=\widetilde{\Omega} \circ v_{2} \Longrightarrow \widetilde{\Omega}\left(v_{1}(t)\right)=\widetilde{\Omega}\left(v_{2}(t)\right) \quad \forall t \in S^{1} .
$$


Como $\widetilde{\Omega}$ é um isomorfismo temos

$$
v_{1}(t)=v_{2}(t) \forall t \in S^{1} \Longrightarrow v_{1}=v_{2} .
$$

Logo, $\widetilde{\Lambda \Omega}$ é injetora.

Seja agora $X \in \Gamma^{\infty}\left(c^{*} T^{*} M\right)$ então $X: S^{1} \rightarrow T^{*} M$ é tal que $\pi(X(t))=c(t)$ para todo $t \in S^{1}$, onde $\pi$ : $T^{*} M \rightarrow M$ é a projeção canônica. Como $X(t) \in T^{*} M$ para todo $t \in S^{1}$ e $\widetilde{\Omega}$ é sobrejetora então existe $u(t) \in$ $T M$ tal que

$$
X(t)=\widetilde{\Omega}(u(t)) \Longrightarrow X=\widetilde{\Omega} \circ u=\widetilde{\Lambda \Omega}(u) .
$$

Logo, $\widetilde{\Lambda \Omega}: T(\Lambda M) \rightarrow \Gamma^{\infty}\left(c^{*} T^{*} M\right)$ é sobrejetora.

Notação 4.36. Dizemos nesse caso que $\Lambda \Omega$ é uma estrutura simplética fraca em $\Lambda M$.

Existe um campo de vetores natural $\mathfrak{D}$ em $\Lambda M$, independente do fato de $\mathrm{M}$ ser simplética, definido por:

$$
\mathfrak{D}(c)=\frac{d c}{d t}
$$

onde $\frac{d c}{d t}: S^{1} \rightarrow T M$ e $\pi\left(\frac{d c}{d t}(t)\right)=c(t)$ para todo $t \in S^{1}$, ou seja, $\frac{d c}{d t} \in T_{c}(\Lambda M)$. Esse campo de vetores é o gerador infinitesimal da ação

$$
\begin{aligned}
\lambda: S^{1} \times \Lambda M & \rightarrow \Lambda M \\
(g, c) & \mapsto g \cdot c
\end{aligned}
$$

onde $(g \cdot c)(t)=c(g+t)$ para todo $t \in S^{1}$.

Lema 4.37. Se $\Omega$ é uma estrutura simplética em $M$ então a ação de $S^{1}$ em $\Lambda M$ preserva a estrutura simplética fraca $\Lambda \Omega$.

Demonstração. A ação de $S^{1}$ em $\Lambda M$ induz uma ação de $S^{1}$ em $T(\Lambda M)$ dada por

$$
\begin{aligned}
\bar{\lambda}: S^{1} \times T(\Lambda M) & \rightarrow T(\Lambda M) \\
(g, v) & \mapsto g \cdot v
\end{aligned}
$$

onde $(g \cdot v)(t)=v(g+t)$ para todo $t \in S^{1}$. Observe que se $v \in T_{c}(\Lambda M)$ então

$$
(g \cdot v)(t)=v(g+t) \Longrightarrow \pi \circ(g \cdot v)(t)=\pi(v(g+t))=c(g+t)=(g \cdot c)(t) \Longrightarrow g \cdot v \in T_{g \cdot c}(\Lambda M) .
$$

Fixado $g \in S^{1}$ queremos mostrar que $\lambda_{g}: \Lambda M \rightarrow \Lambda M$ definido por $\lambda_{g}(c)=\lambda(g, c)=g \cdot c$ é um simplectomorfismo. De fato, se $g \cdot v_{1}, g \cdot v_{2} \in T_{\lambda_{g}(c)}(\Lambda M)$ temos

$$
\begin{aligned}
\left\langle\Lambda \Omega,\left(g \cdot v_{1}, g \cdot v_{2}\right)\right\rangle & =\int_{0}^{1}\left\langle\Omega((g \cdot c)(t)),\left(g \cdot v_{1}(t), g \cdot v_{2}(t)\right)\right\rangle d t \\
& =\int_{0}^{1}\left\langle\Omega(c(g+t)),\left(v_{1}(g+t), v_{2}(g+t)\right)\right\rangle d t \\
& =\int_{0}^{1}\left\langle\Omega(c(s)),\left(v_{1}(s), v_{2}(s)\right)\right\rangle d s \\
& =\left\langle\Lambda \Omega,\left(v_{1}, v_{2}\right)\right\rangle
\end{aligned}
$$

onde $s=g+t$ e $v_{1}, v_{2} \in T_{c}(\Lambda M)$.

Corolário 4.38. $\mathfrak{D}$ é localmente um campo de vetores Hamiltoniano.

Demonstração. Sejam

$$
i_{\mathfrak{D}} \Lambda \Omega:=\Lambda \Omega(\mathfrak{D}, \cdot)
$$


e $\phi_{t}$ o fluxo em $\Lambda M$ tal que $\left.\frac{d}{d t} \varphi_{t}\right|_{t=0}=\mathfrak{D}$. Como $\phi_{t}$ preserva $\Lambda \Omega$ (Lema 4.37) e $\Lambda \Omega$ é fechada (Lema 4.34) segue-se que $d\left(i_{\mathfrak{D}} \Lambda \Omega\right)=0$. Portanto $i_{\mathfrak{D}} \Lambda \Omega$ é fechada e pelo Lema de Poincaré essa 1-forma é localmente exata, isto é, existe $\mathfrak{F}$ tal que

$$
\Lambda \Omega(\mathfrak{D}, \cdot)=i_{\mathfrak{D}} \Lambda \Omega=d \mathfrak{F} .
$$

Portanto, $\mathfrak{D}$ é um campo localmente hamiltoniano.

Podemos encontrar uma função polivalente geradora para $\mathfrak{D}$ integrando a 1-forma fechada $\widetilde{\Lambda \Omega} \circ \mathfrak{D}$ ao longo de um caminho suave. Se $\left\{c_{s}\right\}$ é um caminho suave temos

$$
\begin{aligned}
\int_{\left\{c_{s}\right\}} \widetilde{\Lambda \Omega} \circ \mathfrak{D} & =\int_{0}^{1}\left\langle\Lambda \Omega \circ \mathfrak{D}, \frac{d c_{s}}{d s}\right\rangle d s \\
& =\int_{0}^{1}\left\langle\Lambda \Omega\left(c_{s}\right),\left(\mathfrak{D}\left(c_{s}\right), \frac{d c_{s}}{d s}\right)\right\rangle d s \\
& =\int_{0}^{1} \int_{0}^{1}\left\langle\Omega\left(c_{s}(t)\right),\left(\mathfrak{D}\left(c_{s}\right)(t), \frac{d c_{s}}{d s}(t)\right)\right\rangle d t d s \\
& =\int_{0}^{1} \int_{0}^{1}\left\langle\Omega\left(c_{s}(t)\right),\left(\frac{d c_{s}}{d t}(t), \frac{d c_{s}}{d s}(t)\right)\right\rangle d t d s
\end{aligned}
$$

Se $C(t, s)=c_{s}(t)$ então $\frac{d c_{s}}{d t}(t)=\frac{\partial C}{\partial t}(t, s)$ e $\frac{d c_{s}}{d s}(t)=\frac{\partial C}{\partial s}(t, s)$ assim,

$$
\int_{\left\{c_{s}\right\}} \widetilde{\Lambda \Omega} \circ \mathfrak{D}=\int_{0}^{1} \int_{0}^{1}\left\langle\Omega(C(t, s)),\left(\frac{\partial C}{\partial t}(t, s), \frac{\partial C}{\partial s}(t, s)\right)\right\rangle d t d s=\int_{C} \Omega .
$$

Se $\Omega$ é exata, sua integral sob o cilindro $\mathrm{C}$ depende apenas dos loops da fronteira e, nesse caso, a função hamiltoniana de $\mathfrak{D}$ será monovalente. Explicitamente, se $\Omega=-d \omega$ para alguma 1-forma $\omega$, segue do Teorema de Stokes que

$$
\int_{\left\{c_{s}\right\}} \widetilde{\Lambda \Omega} \circ \mathfrak{D}=\int_{C} \Omega=-\int_{C} d \omega=-\int_{\partial C} \omega
$$

Como $\partial C=C_{0}-C_{1}$ então

$$
\int_{\left\{c_{s}\right\}} \widetilde{\Lambda \Omega} \circ \mathfrak{D}=\int_{\partial c_{1}} \omega-\int_{\partial c_{0}} \omega
$$

Assim, se definirmos $\mathfrak{F}: \Lambda M \rightarrow \mathbb{R}$ por $\mathfrak{F}(c)=\int_{c} \omega$ então $d \mathfrak{F}=\widetilde{\Lambda \Omega}(\mathfrak{D})$ e $\mathfrak{F}$ é a função hamiltoniana global de $\mathfrak{D}$. Observe que $\mathfrak{F}$ depende da escolha de $\omega$, mas é determinada a menos de constante por cada componente de $\Lambda M$.

Em geral, $\Omega$ não é exata, assim $\mathfrak{F}$ não pode ser definida (como uma função monovalente) exceto em subconjuntos simplesmente conexos de $\Lambda M$ (ou no recobrimento universal de $\Lambda M$ ). Com essa ressalva continuaremos usando $d \mathfrak{F}=\widehat{\Lambda \Omega}(\mathfrak{D})$.

Teorema 4.39. Seja $M$ uma variedade simplética e $H: M \rightarrow \mathbb{R}$ uma função hamiltoniana. Então, existe uma função $Q: \Lambda M \rightarrow \mathbb{R}$ tal que os pontos críticos de $Q$ são órbitas periódicas, com período $\tau>0$, do campo hamiltoniano $X_{H}$.

Demonstração. Se H é uma função real em $\mathrm{M}$, o campo de vetores $X_{H}$ pode ser levantado em um campo de vetores $\Lambda X_{H}$ em $\Lambda M$ definido por

$$
\Lambda X_{H}(c)=X_{H} \circ c .
$$


Como

$$
\begin{aligned}
\Lambda \Omega\left(\Lambda X_{H}(c), \cdot\right) & =\Lambda \Omega\left(X_{H} \circ c, \cdot\right) \\
& =\int_{0}^{1} \Omega(c(t))\left(X_{H}(c(t)), \cdot\right) d t \\
& =\int_{0}^{1} d H(c(t)) d t \\
& =d\left(\int_{0}^{1} H(c(t)) d t\right) \\
& =d(\Lambda H(c))
\end{aligned}
$$

então $\Lambda H$ pode ser vista como a função hamiltoniana para $\Lambda X_{H}$, isto é, $X_{\Lambda H}=\Lambda X_{H}$.

Um loop $c \in \Lambda M$ é a uma órbita de $X_{H}$ quando $\frac{d c}{d t}=X_{H} \circ c$, isto é, quando $\mathfrak{D}(c)=\Lambda X_{H}$. Assim, as órbitas periódicas em $M$ com período 1 para $X_{H}$ são os zeros em $\Lambda M$ do campo de vetores $\mathfrak{D}-\Lambda X_{H}$ ou da 1-forma $\widetilde{\Lambda \Omega} \circ\left(\mathfrak{D}-\Lambda X_{H}\right)=d \mathfrak{F}-d \Lambda H$. Quando $\mathfrak{F}$ é monovalente podemos escrever a 1-forma $d \mathfrak{F}-d \Lambda H$ como $d(\mathfrak{F}-\Lambda H)$. Assim, nesse caso as órbitas periódicas são os pontos críticos da função $\mathfrak{F}-\Lambda H: \Lambda M \rightarrow \mathbb{R}$.

Para identificar as órbitas periódicas de $X_{H}$ com período $\tau>0$ podemos ver que elas correspondem, após mudança de coordenadas (no tempo), aos loops $c \in \Lambda M$ para os quais $\frac{d c}{d t}=\tau X_{H} \circ c$. Estes são zeros do campo de vetores

$$
\mathfrak{D}-\tau \Lambda X_{H}
$$

ou zeros da 1-forma

$$
d \mathfrak{F}-\tau d \Lambda H
$$

ou pontos críticos da função

$$
\mathfrak{F}-\tau \Lambda H .
$$

Observação 4.40. Quando $M=\mathbb{R}^{2 n}$ com coordenadas $\left(p_{1}, \ldots, p_{n}, q_{1}, \ldots, q_{n}\right)$ e $\Omega=\sum d p_{i} \wedge d q_{i}$, podemos tomar $\omega=\sum q_{i} d p_{i}$ e as soluções das equações de Hamilton

$$
\frac{d p_{i}}{d t}=\frac{\partial H}{\partial q_{i}} \quad \frac{d q_{i}}{d t}=-\frac{\partial H}{\partial p_{i}}
$$

são os extremos do funcional

$$
\int\left(\sum q_{i} d p_{i}-H d t\right)
$$

Esse é precisamente o princípio de Hamilton.

Ainda com a notação do Teorema 4.39, podemos fazer com que todas as órbitas periódicas de $X_{H}$ tenham períodos positivos e sejam os zeros de uma única 1-forma fechada (ou pontos críticos de uma única função) se nos restringirmos um nível de energia particular $H^{-1}(E), E \in \mathbb{R}$. Dado $E \in \mathbb{R}$, defina a função $\psi_{E}^{H}: \Lambda M \times \mathbb{R}^{+} \rightarrow \mathbb{R}$ por

$$
\psi_{E}^{H}(c, \tau)=[\mathfrak{F}-\tau \Lambda(H-E)](c) .
$$

Essa função pode ser polivalente, mas a diferencial $d \psi_{E}^{H}$ dada por

$$
\left\langle d \psi_{E}^{H}(c, \tau),(v, a)\right\rangle=\langle d \mathfrak{F}(c), v\rangle-\langle\tau d \Lambda H(c), v\rangle-a \Lambda(H-E)(c)
$$

é uma 1-forma fechada bem-definida em $\Lambda M \times \mathbb{R}^{+}$. A forma é zero quando:

(i) $(d \mathfrak{F}-\tau d \Lambda H)(c)=0 \mathrm{e}$

(ii) $\Lambda(H-E)(c)=0$.

Quando vale a condição (i), $c$ é uma órbita reparametrizada de $X_{H}$ (de período $\tau$ ), assim $H \circ c$ é constante, e a condição (ii) diz que o valor dessa constante é $E$. 
Teorema 4.41. Seja $(M, g)$ uma variedade Riemanniana (ou Finsler) tal que todas as geodésicas de M são fechadas de mesmo período $\tau>0$. Então, existe uma função $\mathfrak{B}: \Lambda M \rightarrow \mathbb{R}$ tal que os pontos críticos de $\mathfrak{B}$ são geodésicas fechadas de período $\tau$ em $M$.

Demonstração. Seja $(M, g)$ uma variedade Riemanniana (ou Finsler) tal que todas as geodésicas de M são fechadas de mesmo período $\tau$. Tome o fibrado cotangente $T^{*} M$, que é sempre uma variedade simplética, e o Hamiltoniano $H: T^{*} M \rightarrow \mathbb{R}$ definido por $H(x, \xi)=\frac{1}{2} g^{i j} \xi_{i} \xi_{j}$. Pelo Teorema 4.39 segue-se que pontos críticos de uma função $Q: \Lambda\left(T^{*} M\right) \rightarrow \mathbb{R}$ são órbitas periódicas de período $\tau$ em $T^{*} M$ do campo $X_{H}$.

Como vimos na Definição 1.34, o fluxo cogeodésico é o fluxo determinado por

$$
\begin{aligned}
& \dot{x}^{i}=\frac{\partial H}{\partial \xi^{i}} \\
& \dot{\xi}^{i}=-\frac{\partial H}{\partial x^{i}}
\end{aligned}
$$

Assim, se $(x(t), \xi(t))$ é uma curva integral do campo hamiltoniano $X_{H}$ segue que $x(t)$ é uma geodésica em M. Logo, como toda geodésica em M tem período $\tau$ segue-se que todas as órbitas de $X_{H}$ tem período $\tau$.

Seja

$$
\Lambda X=\left\{c: S^{1} \rightarrow T^{*} M ; c(t)=(x(t), \xi(x)) \text { satisfaz (4.22) }\right\} .
$$

Então, como todo elemento $c \in \Lambda X$ é uma órbita periódica de período $\tau$ do campo $X_{H}$ temos

$$
d Q(c)=0 \text { para todo } c \in \Lambda X .
$$

Portanto, $\Lambda X$ é o subconjunto de $\Lambda\left(T^{*} M\right)$ em que as órbitas periódicas de $X_{H}$ pertencem.

Seja $\mathscr{L}_{H}: T^{*} M \rightarrow T M$ a transformada de Legendre como na Definição 1.23. Como visto na Seção 1.2.2

$$
\sqrt{2 H(x, \xi)}=\sqrt{g^{i j} \xi_{i} \xi_{j}}
$$

é uma norma Riemanniana (ou co-norma Finsler), então, pelo Teorema 1.25 segue-se que a transformada de Legendre associada a função

$$
\frac{1}{2}[\sqrt{2 H(x, \xi)}]^{2}=H(x, \xi)
$$

é uma bijeção, isto é, $\mathscr{L}_{H}: T^{*} M \rightarrow T M$ é uma bijeção.

Seja $c=(x, \xi) \in \Lambda X$, então, por definição de fluxo geodésico segue-se que $x(t)$ é uma geodésica de $\mathrm{M}$. E como

$$
\begin{aligned}
\mathscr{L}_{H} \circ c(t) & =\mathscr{L}_{H}(x(t), \xi(t)) \\
& =\left(x(t), \frac{\partial H}{\partial \xi_{i}}(x(t), \xi(t))\right) \\
& =(x(t), \dot{x}(t))
\end{aligned}
$$

segue-se que a transformada de Legendre identifica curvas em $\Lambda X$ com curvas do fluxo geodésico em $T M$. Defina $\Lambda \mathscr{L}_{H}: \Lambda(T M) \rightarrow \Lambda\left(T^{*} M\right)$ por $\Lambda \mathscr{L}_{H}(c)=\mathscr{L}_{H}{ }^{-1} \circ c$ e $G: \Lambda M \rightarrow \Lambda(T M)$ por $G(\gamma)=(\gamma, \dot{\gamma})$. Assim, se $\gamma$ é uma geodésica fechada de período $\tau$ em $M$ temos

$$
\begin{aligned}
\left(\Lambda \mathscr{L}_{H} \circ G\right)(\lambda(t)) & =\Lambda \mathscr{L}_{H}(\gamma(t), \dot{\gamma}(t)) \\
& =\mathscr{L}_{H}^{-1}(\gamma(t), \dot{\gamma}(t)) \\
& =(\gamma(t), \xi(t))
\end{aligned}
$$

onde $(\gamma(t), \xi(t))$ é uma solução das equações em (4.22), ou seja, $\left(\Lambda \mathscr{L}_{H} \circ G\right)(\gamma) \in \Lambda X$. Logo, se $\gamma$ é uma geodésica fechada de período $\tau$ em $M$ segue-se pela equação (4.23) que

$$
d\left[Q \circ \Lambda \mathscr{L}_{H} \circ G\right](\gamma)=d Q\left(\Lambda \mathscr{L}_{H} \circ G(\gamma)\right) \cdot d\left[\Lambda \mathscr{L}_{H} \circ G(\gamma)\right]=0 .
$$


Portanto, pontos críticos da função $\mathfrak{B}:=Q \circ \Lambda \mathscr{L}_{H} \circ G: \Lambda M \rightarrow \mathbb{R}$ são geodésicas fechadas de período $\tau$ em M.

\subsubsection{Bifurcação de variedades críticas}

De agora em diante, estudaremos sistemas hamiltonianos que estão próximos de um que tem uma variedade de órbitas periódicas. Isso nos permitirá encontrar uma subvariedade de dimensão finita em $\Lambda M \times \mathbb{R}^{+}$ na qual os pontos críticos de $\psi_{E}^{H}$ estão contidos. O processo de encontrar essa subvariedade de dimensão finita é as vezes chamado método de bifurcação de Liapunov-Schmidt.

Também substituiremos a variedade $C^{\infty}$ de loops por uma variedade Banach de loops tendo um grau finito de diferenciabilidade. Por simplicidade escolhemos a variedade de aplicações $C^{1}$ de $S^{1}$ em M. Nesse caso, $\Lambda M$ denotará essa variedade Banach. Assim:

- O espaço tangente $T_{c} \Lambda M$ é agora o espaço $\Gamma^{1}\left(c^{*} T M\right)$ dos campos de vetores $C^{1}$ ao longo de $c$.

- O espaço cotangente $T_{c}^{*} \Lambda M$ é o espaço $\Gamma^{-1}\left(c^{*} T M\right)$ de distribuições de ordem 1 em $S^{1}$ com valores em $c^{*} T M$.

- A aplicação fibrado $\widetilde{\Lambda \Omega}$ aplica $T_{c} \Lambda M$ injetivamente no subespaço denso $\Gamma^{1}\left(c^{*} T^{*} M\right) \operatorname{de} \Gamma^{-1}\left(c^{*} T M\right)=$ $T_{c}^{*} \Lambda M$. Assim, $\Lambda \Omega$ é uma estrutura simplética fraca em $\Lambda M$.

- O grupo $S^{1}$ ainda age de forma contínua em $\Lambda M$ por difeomorfismos, mas como estamos supondo que o grau de diferenciabilidade das curvas é finito, então essa ação não é mais suave. Como a derivada de um loop $C^{1}$ é, em geral, somente um campo de vetores $C^{0}$, o gerador infinitesimal $\mathfrak{D}$ toma valores no "super fibrado" $\widehat{T} \Lambda M \subset T \Lambda M$ onde para cada $c \in \Lambda M, \widehat{T}_{c} \Lambda M$ é o espaço $\Gamma^{0}\left(c^{*} T M\right)$ de campos de vetores contínuos ao longo de $c$.

- O covetor $\widetilde{\Lambda \Omega} \circ \mathfrak{D}$ ainda pertence ao espaço cotangente $T_{c}^{*} \Lambda M$. Na verdade, $\Lambda \Omega(c): \Gamma^{1}\left(c^{*} T M\right) \rightarrow$ $\Gamma^{1}\left(c^{*} T^{*} M\right)$ se estende a uma aplicação $\Gamma^{0}\left(c^{*} T M\right) \rightarrow \Gamma^{0}\left(c^{*} T^{*} M\right)$ que ainda é um subespaço de $\Gamma^{-1}\left(c^{*} T M\right)=T_{c}^{*} \Lambda M$. Assim, $\widetilde{\Lambda \Omega} \circ \mathfrak{D}$ é uma seção suave do subfibrado $\check{T}^{*} \Lambda M \subset T^{*} \Lambda M$, onde para cada $c \in \Lambda M, \check{T}_{c}^{*} \Lambda M=\Gamma^{0}\left(c^{*} T^{*} M\right)$.

- $d \mathfrak{F}=\widetilde{\Lambda \Omega} \circ \mathfrak{D}$ continua o mesmo e $\mathfrak{F}$ é definida exatamente como antes quando $\Omega$ é exata ou em subconjuntos simplesmente conexos de $\Lambda M$.

De modo geral, se B é uma variedade modelada em um espaço de Banach reflexivo, $\check{T}^{*} B$ é um fibrado vetorial sobre B e $i: \check{T}^{*} B \rightarrow T^{*} B$ é uma aplicação injetiva de fibrados com imagem densa temos

Definição 4.42. Uma 1-forma $\alpha: B \rightarrow T^{*} B$ é hiperregular se existe uma seção suave $\check{\alpha}: B \rightarrow \check{T}^{*} B$ tal que $i \circ \check{\alpha}=\alpha$.

Definição 4.43. Uma subvariedade $\Sigma \subseteq B$ é chamada uma variedade zero para uma 1-forma fechada $\alpha$, se $\alpha(b)=0$ para todo $b \in \Sigma$. Uma variedade zero para $d f$ é chamada uma variedade crítica para a função $f$.

Definição 4.44. Uma variedade zero $\Sigma$ para uma 1-forma fechada hiperregular $\alpha$ em $B$ é chamada de fracamente não degenerada se, para cada $b \in B, D_{b} \check{\alpha}$ tem imagem fechada e

$$
\operatorname{ker}\left(D_{b} \check{\alpha}\right)^{*}=i_{b}^{*}\left(T_{b} \Sigma\right) .
$$

Teorema 4.45. Seja $\Sigma \subseteq B$ uma variedade zero compacta, fracamente não degenerada para a 1-forma fechada, hiperregular $\alpha$ e seja phi outra 1-forma hiperregular. Então, existe uma vizinhança $\mathscr{U}$ de $\Sigma$ em $B$ e um número $\varepsilon_{0}>0$ tal que para $|\varepsilon|<\varepsilon_{0}$, existe um mergulho $e_{\varepsilon}: \Sigma \rightarrow \mathscr{U}$ tal que o conjunto zero de $\alpha+\varepsilon \phi$ em $\mathscr{U}$ é o conjunto zero do pullback de $\alpha+\varepsilon \phi$ para $e_{\varepsilon}(\Sigma)$.

Se $\phi$ é exata, então $\alpha+\varepsilon \phi$ é exata em $e_{\varepsilon}(\Sigma)$. Assim, o conjunto zero de $\alpha+\varepsilon \phi$ em $\mathscr{U}$ é o conjunto crítico de uma função em $e_{\varepsilon}(\Sigma)$. 
Seja $\mathrm{M}$ uma variedade de dimensão finita, $\Omega$ uma estrutura simplética em $\mathrm{M}, H$ uma função real em M, B a variedade de Banach $\Lambda M \times \mathbb{R}^{+}, \check{T}^{*} B$ o subfibrado $\check{T}^{*} \Lambda M \times T^{*} \mathbb{R}^{+}$de $T * B, i: \check{T}^{*}\left(\Lambda M \times \mathbb{R}^{+}\right) \rightarrow$ $T^{*}\left(\Lambda M \times \mathbb{R}^{+}\right)$a inclusão e $d \psi_{E}^{H}$ a diferencial do funcional hamiltoniano como definida em (4.21).

Definição 4.46. Uma variedade zero fracamente não degenerada $\Sigma \subseteq \Lambda M \times \mathbb{R}^{+}$para $d \psi_{E}^{H}$ é chamada uma variedade periódica não degenerada com energia $E$ para o sistema hamiltoniano $(M, \Omega, H)$ se nenhuma das curvas em $\Sigma$ são curvas ponto, isto é, se nenhuma das curvas em $\Sigma$ passam pelos pontos críticos de $H$.

Observação 4.47. Se $\mathrm{H}$ é uma função suave em $\mathrm{M}$, então $\Lambda H$ é uma função suave em $\Lambda M$, assim $d \Lambda H$ é uma seção suave de $T^{*} \Lambda M$. Os valores de $d \Lambda H$ em $c \in \Lambda M$ são $\Lambda d H(c)=d H \circ c$ e pertencem ao subespaço $\Gamma^{1}\left(c^{*} T^{*} M\right)$ de $\Gamma^{0}\left(c^{*} T^{*} M\right)$, assim, $d \Lambda H$ é uma seção suave de $\check{T}^{*} \Lambda M$. Podemos concluir disso que a 1 -forma fechada $d \psi_{E}^{H}$, onde

$$
d \psi_{E}^{H}(c, \tau)=d \mathfrak{F}(c)-\tau d \Lambda H(c)-\Lambda\left(H_{E}\right)(c),
$$

é uma seção suave do subfibrado denso $\check{T}^{*} \Lambda M \times T^{*} \mathbb{R}^{+}$de $T^{*} M \times T^{*} \mathbb{R}^{+}=T^{*}\left(\Lambda M \times \mathbb{R}^{+}\right)$. Portanto, $d \psi_{E}^{H}$ é uma 1-forma hiperregular.

Teorema 4.48. Seja $\Sigma \subseteq \Lambda M \times \mathbb{R}^{+}$uma variedade periódica compacta, não degenerada para o sistema Hamiltoniano $(M, \Omega, H)$ e seja $H_{1}$ qualquer função em $M$. Então, existe uma vizinhança $\mathscr{U}$ de $\Sigma$ em $\Lambda M \times$ $\mathbb{R}^{+}$e um número $\varepsilon_{0}>0$ tal que para $|\varepsilon| \leq \varepsilon_{0}$, o número de órbitas periódicas em $\mathscr{U}$ para o sistema Hamiltoniano $\left(M, \Omega, H+\varepsilon H_{1}\right)$ é igual ao número de pontos críticos de alguma função definida em $\Sigma$.

Demonstração. Pela definição de variedade periódica não degenerada segue que $\Sigma$ é uma variedade zero fracamente não degenerada da 1 -forma $d \psi_{E}^{H}$ em $\Lambda M \times \mathbb{R}^{+}$e pela Observação 4.47 segue que $d \psi_{E}^{H}$ é hiperregular. Como pela equação (4.21) temos

$$
\begin{aligned}
\left\langle d \psi_{E}^{H+\varepsilon H_{1}}(c, \tau),(v, a)\right\rangle & =\langle d \mathfrak{F}(c), v\rangle-\tau\left\langle d \Lambda\left(H+\varepsilon H_{1}\right)(c), v\right\rangle-a \Lambda\left(H+\varepsilon H_{1}-E\right)(c) \\
& =\langle d \mathfrak{F}(c), v\rangle-\tau\left\langle d\left(\Lambda H+\varepsilon \Lambda H_{1}\right)(c), v\right\rangle-a\left[\Lambda(H-E)(c)+\varepsilon \Lambda H_{1}(c)\right] \\
& =\langle d \mathfrak{F}(c), v\rangle-\tau\langle d \Lambda H(c), v\rangle-a \Lambda(H-E)(c)-\tau \varepsilon\left\langle d \Lambda H_{1}(c), v\right\rangle-a \varepsilon \Lambda H_{1}(c) \\
& =\left\langle d \psi_{E}^{H}(c, \tau),(v, a)\right\rangle+\varepsilon\left(-\tau\left\langle d \Lambda H_{1}(c), v\right\rangle-a \Lambda H_{1}(c)\right) \\
& =\left\langle d \psi_{E}^{H}(c, \tau),(v, a)\right\rangle+\varepsilon\left\langle d \Lambda H^{1}(c, \tau),(v, a)\right\rangle
\end{aligned}
$$

onde $\Lambda H^{1}: \Lambda M \times \mathbb{R}^{+} \rightarrow \mathbb{R}$ é a função definida por

$$
\Lambda H^{1}(c, \tau)=-\tau \Lambda H_{1}(c)
$$

segue que $d \psi_{E}^{H+\varepsilon H_{1}}=d \psi_{E}^{H}+\varepsilon d \Lambda H^{1}$, onde $d \Lambda H^{1}$ é exata. Assim, pelo o Teorema 4.45 concluímos que para $\varepsilon$ suficientemente pequeno existe um mergulho $e_{\varepsilon}: \Sigma \rightarrow \mathscr{U}$ tal que o conjunto zero de $d \psi_{E}^{H+\varepsilon H_{1}}$ em $\mathscr{U}$ é o conjunto de pontos críticos de uma função $f$ em $e_{\varepsilon}(\Sigma)$. Além disso, como $e_{\varepsilon}$ é um mergulho, se $p=e_{\varepsilon}(q)$ e $q$ é um ponto crítico de $f \circ e_{\varepsilon}$ temos

$$
0=d\left(f \circ e_{\varepsilon}\right)(q)=d f\left(e_{\varepsilon}(q)\right) \cdot d e_{\varepsilon}(q)=d f(p) \cdot d e_{\varepsilon}(q) \Longrightarrow d f(p)=0 .
$$

Por outro lado, se $p=e_{\varepsilon}(q)$ é um ponto crítico de $f$ temos

$$
d\left(f \circ e_{\varepsilon}\right)(q)=d f(p) \cdot d e_{\varepsilon}(q)=0 .
$$

Logo, os pontos críticos de $f \circ e_{\varepsilon}$ correspondem aos pontos críticos de $f$. Portanto, o conjunto zero de $d \psi_{E}^{H+\varepsilon H_{1}}$ em $\mathscr{U}$ corresponde ao conjunto de pontos críticos de uma função em $\Sigma$.

Observação 4.49. É possível mostrar que $\varepsilon_{0}$ depende somente do $C^{1}$ tamanho de $H_{1}$.

Definição 4.50. Denote por $T_{1}^{*} M$ o subconjunto de $T^{*} M$ tal que

$$
T_{1}^{*} M=\left\{(x, \xi) \in T^{*} M ;\|\xi\|_{g}=1\right\} .
$$


Definição 4.51. A ação de $S^{1}$ em $T_{1} M$, como definida na equação (1.29), induz uma ação de $S^{1}$ em $T_{1}^{*} M$ como descreveremos a seguir. Se $s \in S^{1}=\mathbb{R} / \mathbb{Z}$ e $(x, \xi) \in T_{1}^{*} M$ então existe $v \in T_{x} M$ tal que $\xi=g(v, \cdot)$. Assim, definimos

$$
s \cdot(x, \xi)=s \cdot(x, g(v, \cdot))=\left(\gamma_{x}^{v}(2 \pi s), \dot{\gamma}_{x}^{v}(2 \pi s)\right)
$$

onde $\gamma_{x}^{v}$ é a geodésica em $\mathrm{M}$ tal que $\gamma_{x}^{v}(0)=x$ e $\dot{\gamma}_{x}^{v}(0)=v$. Assim, $C^{*}:=T_{1}^{*} M / S^{1}$ é o quociente de $T_{1}^{*} M$ pela ação desse grupo.

Corolário 4.52. Seja $(M, g)$ uma variedade Riemanniana compacta onde todas as geodésicas são fechadas e tem mesmo período $2 \pi$. Qualquer métrica Finsler $F$ suficientemente próxima da métrica Riemanniana $g$ em $M$ tem tantas geodésicas fechadas de comprimento próximo de $2 \pi$ quanto uma função em $C^{*}=T_{1}^{*} M / S^{1}$ tem pontos críticos.

Demonstração. Como $C^{*}$ é o espaço das geodésicas de $\mathrm{M}$, vistas em $T_{1}^{*} M$, segue que $C^{*} \subset \Lambda\left(T^{*} M\right)$. Além disso, como $C^{*} \times\{2 \pi\} \subset \Lambda\left(T^{*} M\right) \times \mathbb{R}^{+}$é uma subvariedade compacta e periódica não degenerada para o sistema Hamiltoniano $\left(T^{*} M, \omega_{\text {can }}, H_{0}\right)$ pelo Teorema 4.48 segue que para qualquer $H_{1}$ definido em $T^{*} M$ existem uma vizinhança $\mathscr{U}$ de $C^{*} \times\{2 \pi\}$ e um número $\varepsilon_{0}>0$ tais que para todo $|\varepsilon| \leq \varepsilon_{0}$ o número de órbitas periódicas em $\mathscr{U}$ para o sistema Hamiltoniano $\left(T^{*} M, \omega_{\mathrm{can}}, H_{0}+\varepsilon H_{1}\right)$ é igual ao número de pontos críticos de alguma função definida em $C^{*} \times\{2 \pi\}$.

Como $H_{\varepsilon}:=H_{0}+\varepsilon H_{1}$ é uma co-norma Finsler se $\varepsilon$ é suficientemente pequeno, então, assumindo que $\varepsilon_{0}$ é pequeno o suficiente para que isso ocorra segue que $F_{\varepsilon}=H_{\varepsilon} \circ \mathscr{L}_{\frac{1}{2} H_{\varepsilon}^{2}}^{-1}$ é uma métrica Finsler para todo $|\varepsilon| \leq \varepsilon_{0}$. Como todo Hamiltoniano na $\varepsilon_{0}$-vizinhança de $H_{0}$ pode ser escrito na forma $H_{0}+\varepsilon H_{1}$ para alguma função $H_{1}$ segue que, para todo $|\varepsilon| \leq \varepsilon_{0}$, o número de geodésicas periódicas de período próximo de $2 \pi$ na métrica Finsler $F_{\varepsilon}$ é igual ao número de pontos críticos de uma função definida em $C^{*} \times\{2 \pi\}$, ou melhor, em $C^{*}$.

Corolário 4.53. Seja $(M, g)$ uma variedade Riemanniana compacta onde todas as geodésicas são fechadas e tem mesmo período $2 \pi$. Qualquer métrica Finsler $F$ suficientemente próxima da métrica Riemanniana $g$ em $M$ tem tantas geodésicas fechadas de comprimento próximo de $2 \pi$ quanto uma função em $C=T_{1} M / S^{1}$ tem pontos críticos.

Antes de demonstrar esse resultado, observe que:

Afirmação 4.54. As variedades $C=T_{1} M / S^{1} e C^{*}=T_{1}^{*} M / S^{1}$ são difeomorfas.

Demonstração. Como sabemos, $f: T_{1} M \rightarrow T_{1}^{*} M$ definida por

$$
f(x, v)=(x, g(v, \cdot))
$$

é um difeomorfismo. Se $\pi: T_{1} M \rightarrow C$ e $p: T_{1}^{*} M \rightarrow C^{*}$ são projeções canônicas defina $A: C \rightarrow C^{*}$ de modo que

$$
\pi(\eta) \mapsto p(f(\eta)) .
$$

Veja que esta aplicação está bem-definida pois se $u_{1}\left(x_{1}, v_{1}\right)$ e $u_{2}\left(x_{2}, v_{2}\right)$ são elementos de $T_{1} M$ tais que $\pi\left(u_{1}\right)=\pi\left(u_{2}\right)$ então para todo $s_{1} \in \mathbb{R}$ existe $s_{2} \in \mathbb{R}$ tal que

$$
\left(\gamma_{x_{1}}^{\nu_{1}}\left(2 \pi s_{1}\right), \dot{\gamma}_{x_{1}}^{\nu_{1}}\left(2 \pi s_{1}\right)\right)=\left(\gamma_{x_{2}}^{\nu_{2}}\left(2 \pi s_{2}\right), \dot{\gamma}_{x_{2}}^{\nu_{2}}\left(2 \pi s_{2}\right)\right) .
$$

E pela unicidade de geodésicas temos $\gamma_{x_{1}}^{\nu_{1}}=\gamma_{x_{2}}^{\nu_{2}}$. Assim,

$$
\begin{aligned}
p\left(f\left(u_{1}\right)\right) & =p\left(x_{1}, g\left(v_{1}, \cdot\right)\right) \\
& =\left(\gamma_{x_{1}}^{v_{1}}, g\left(\dot{\gamma}_{x_{1}}^{v_{1}}, \cdot\right)\right) \\
& =\left(\gamma_{x_{2}}^{v_{2}}, g\left(\dot{\gamma}_{x_{2}}^{v_{2}}, \cdot\right)\right) \\
& =p\left(x_{2}, g\left(v_{2}, \cdot\right)\right) \\
& =p\left(f\left(u_{2}\right)\right)
\end{aligned}
$$


Além disso, A é uma função suave já que $p$ e $f$ são suaves. Da mesma forma, se $B: C^{*} \rightarrow C$ definida de modo que

$$
p(u) \mapsto \pi\left(f^{-1}(u)\right)
$$

segue que $\mathrm{B}$ esta bem-definida e é suave. Portanto, como $A \circ B=\operatorname{Id}$ e $B \circ A=\operatorname{Id}$ segue que $C$ e $C^{*}$ são difeomorfos.

Demonstração do Corolário 4.53. Veja que pela Afirmação 4.54 existe um difeomorfismo $h$ entre $C$ e $C^{*}$ e pelo Corolário 4.52 existe uma função $f$ definida em $C^{*}$ tal que os pontos críticos de $f$ correspondem as geodésicas fechadas de comprimento próximo de $2 \pi$ numa métrica Finsler $F$ suficientemente próxima de $g$.

Observe também que se $p=h(q)$ é um ponto crítico de $f$ temos

$$
d(f \circ h)(q)=d f(h(q)) \cdot d h(q)=d f(p) \cdot d h(q)=0
$$

e se $x$ é um ponto crítico de $f \circ h$ temos

$$
0=d(f \circ h)(x)=d f(h(x)) \cdot d h(x)
$$

e como $d h(x)$ é um isomorfismo segue que $d f(h(x))=0$, isto é, $h(x)$ é um ponto crítico de $f$. Logo,

$\{$ pontos críticos de $f\} \simeq\{$ pontos críticos de $f \circ h\}$.

Portanto, $f \circ h$ é a função em $C$ tal que os seus pontos críticos correspondem as geodésicas fechadas de comprimento próximo de $2 \pi$ numa métrica Finsler $F$ suficientemente próxima de $g$. 


\section{Capítulo 5}

\section{Exemplos de Katok com quantidade finita de geodésicas fechadas}

\subsection{Construção de Métricas Finsler e caracterização de geodésicas fechadas}

Seja M uma variedade diferenciável. Para obter os exemplos de Katok comecemos com uma métrica Riemanniana $g$ em M com todas as geodésicas fechadas e que admite um grupo a um parâmetro de isometrias $\phi_{t}$.

Teorema 5.1 (Wadsley, [Bes12], pág 183). Se as órbitas de um fluxo em uma variedade Riemanniana são geodésicas parametrizadas por comprimento de arco, então, o fluxo é periódico, de modo que as órbitas tem um período comum.

Pelo teorema acima as geodésicas de $g$ tem um período comum $r$ e podemos normalizar a métrica para que $r=2 \pi$. Após mudar o subgrupo a um parâmetro $\phi_{t}$ se necessário, podemos assumir também que $\phi_{2 \pi}=I d$.

Lema 5.2. Seja $H_{\alpha}: T^{*} M \rightarrow \mathbb{R}$ uma função hamiltoniana definida por

$$
H_{\alpha}=H_{0}+\alpha H_{1}
$$

onde $H_{0}$ e $H_{1}$ são como em (4.9) e (4.5), respectivamente. Se $\alpha$ é suficientemente pequeno então $H_{\alpha}$ é uma co-norma Finsler não-simétrica.

Demonstração. Claramente vemos que $H_{\alpha}$ é $C^{\infty}$ em $T^{*} M \backslash\{0\}$ e positivamente homogênea de grau 1, então, precisamos mostrar apenas que a forma bilinear associada a $H_{\alpha}$ é positiva definida para todo $\eta \in T^{*} M$. Note que como $H_{\alpha}$ é positivamente homogênea de grau 1, pela Observação 1.4 segue que

$$
g_{i j}^{\alpha}:=\frac{1}{2} \frac{\partial^{2} H_{\alpha}^{2}}{\partial \xi_{i} \partial \xi_{j}}
$$

é positivamente homogênea de grau 0 , isto é,

$$
\left.g_{i j}^{\alpha}\right|_{(x, \lambda \xi)}=\left.g_{i j}^{\alpha}\right|_{(x, \xi)}
$$

para todo $(x, \xi) \in T^{*} M \backslash\{0\}$ e $\lambda>0$. Sejam $\varepsilon>0$ e $\mathscr{H}: T_{1}^{*} M \times(-\varepsilon, \varepsilon) \rightarrow \mathbb{R}$ uma função dada por $\mathscr{H}(\eta, \alpha)=H_{\alpha}(\eta)$. Como $H_{\alpha}$ é $C^{\infty}$ em $T^{*} M \backslash\{0\}$ então $\mathscr{H}$ também é $C^{\infty}$. Assim,

$$
h(\eta, \alpha):=g_{i j}^{\alpha}(\eta)
$$

varia suavemente em $T_{1}^{*} M \times(-\varepsilon, \varepsilon)$. Além disso, como

$$
\operatorname{det}\left(g_{i j}^{0}(\eta)\right)>0
$$


sempre que $\eta \neq 0$ temos

$$
\operatorname{det}(h(\eta, 0))>0
$$

para todo $\eta \in T_{1}^{*} M$. Como det $\circ$ é uma função contínua, para todo $\eta \in T_{1}^{*} M$ existe uma vizinhança aberta $U \times\left(-\lambda_{U}, \lambda_{U}\right)$ de $(\eta, 0)$ onde $0<\lambda_{U} \leq \varepsilon$ tal que

$$
\operatorname{det} \circ h(\rho, \alpha)>0 \text {, para todo }(\rho, \alpha) \in U \times\left(-\lambda_{U}, \lambda_{U}\right) .
$$

Como os abertos $\{U\}$ formam uma cobertura para o compacto $T_{1}^{*} M$, então existe uma subcobertura finita $\left\{U_{1}, \cdots, U_{k}\right\}$ que também cobre $T_{1}^{*} M$. Logo, tomando $\lambda:=\min \left\{\lambda_{U_{i}} ; 1 \leq i \leq k\right\}$ segue que $\lambda>0 \mathrm{e}$

$$
\operatorname{det} \circ h(\eta, \alpha)>0 \text {, para todo }(\eta, \alpha) \in T_{1}^{*} M \times(-\lambda, \lambda) \text {. }
$$

Logo, por (5.2) obtemos

$$
\operatorname{det}\left(g_{i j}^{\alpha}(\eta)\right)>0, \text { para todo } \eta \in T^{*} M \backslash\{0\}
$$

se $\alpha \in(-\lambda, \lambda)$, isto é, $g_{i j}^{\alpha}(\eta)$ é uma forma positiva definida para todo $\eta \in T^{*} M \backslash\{0\}$ e $\alpha \in(-\lambda, \lambda)$. Portanto, $H_{\alpha}$ é uma co-norma Finsler se $\alpha \in(-\lambda, \lambda)$. Além disso, como $H_{1}(p, \xi) \neq H_{1}(p,-\xi)$ segue que $H_{\alpha}$ é não simétrica.

Observação 5.3. Note que a função $H_{1}$ não foi fundamental para a demonstração do Lema 5.2. Logo, por essa mesma demonstração segue que para qualquer $H_{1}: T^{*} M \rightarrow \mathbb{R}$ positivamente homogêneo de grau $1 \mathrm{e}$ $C^{\infty}$ em $T^{*} M \backslash\{0\}$ a função $H_{\alpha}=H_{0}+\alpha H_{1}$ é uma co-norma Finsler, se $\alpha$ é suficientemente pequeno.

Corolário 5.4. Seja $F_{\alpha}: T M \rightarrow \mathbb{R}$ definida por

$$
F_{\alpha}=H_{\alpha} \circ \mathscr{L}_{\frac{1}{2} H_{\alpha}^{2}}^{-1}
$$

Se $\alpha$ é suficientemente pequeno, então, $F_{\alpha}$ é uma métrica Finsler não simétrica.

Demonstração. Como $H_{\alpha}$ é uma co-norma Finsler se $\alpha$ é suficientemente pequeno (Lema 5.2) então pelo Teorema 1.25 segue que $F_{\alpha}$ é uma métrica Finsler se $\alpha$ é suficientemente pequeno. Além disso, $F_{\alpha}$ é não simétrica pois $H_{\alpha}$ é não simétrica.

Lema 5.5. As curvas integrais dos campos $X_{\frac{1}{2} H_{0}^{2}}$ e $X_{H_{0}}$ são reparametrizações uma da outra.

Demonstração. Pelo Teorema 4.29, $X_{H_{0}}$ não corresponde ao fluxo co-geodésico de g mas $X_{H_{0}}$ e $X_{\frac{1}{2} H_{0}^{2}}$ são proporcionais uma vez que

$$
\omega\left(X_{\frac{1}{2} H_{0}^{2}}, \cdot\right)=d\left(\frac{1}{2} H_{0}^{2}\right)=H_{0} d H_{0}=H_{0} \omega\left(X_{H_{0}}, \cdot\right)=\omega\left(H_{0} X_{H_{0}}, \cdot\right)
$$

o que implica que

$$
X_{\frac{1}{2} H_{0}^{2}}=H_{0} \cdot X_{H_{0}} .
$$

Logo, as curvas integrais dos campos $X_{\frac{1}{2} H_{0}^{2}}$ e $X_{H_{0}}$ são reparametrizações uma da outra.

Ainda sobre esses campos podemos afirmar que:

Lema 5.6. Se $\gamma(t)$ é uma geodésica de $g$, $(\gamma(t), Y(\gamma(t)))$ uma curva integral de $X_{\frac{1}{2} H_{0}^{2}}$ em $T^{*} M$, onde $Y(\gamma(t))=g(\dot{\gamma}(t), \cdot)$, e $\gamma_{0}$ a geodésica $\gamma$ parametrizada por comprimento de arco, então, $\left(\gamma_{0}(t), Y\left(\gamma_{0}(t)\right)\right)$ é uma curva integral de $X_{H_{0}}$.

Demonstração. Se $c(t)=(\gamma(t), Y(\gamma(t)))$ segue pela definição de curva integral que $\dot{c}(t)=X_{\frac{1}{2} H_{0}^{2}}(c(t))$. Como

$$
\gamma_{0}(t)=\gamma\left(\frac{t}{\mu}\right), \text { onde } \mu^{2}=g(\dot{\gamma}(t), \dot{\gamma}(t))
$$

temos

$$
c_{0}(s):=\left(\gamma_{0}(s), Y\left(\gamma_{0}(s)\right)\right)=\left(\gamma\left(\frac{s}{\mu}\right), Y\left(\gamma\left(\frac{s}{\mu}\right)\right)\right)=c\left(\frac{s}{\mu}\right) .
$$


Observe que

$$
H_{0}(\gamma(t), Y(\gamma(t)))=H_{0}(\gamma(t), g(\dot{\gamma}(t), \cdot))=\sqrt{g(\dot{\gamma}(t), \dot{\gamma}(t))}=\mu .
$$

Assim, pelas equações (5.5) e (5.4) temos

$$
\begin{aligned}
\dot{c_{0}}(s) & =\frac{1}{\mu} \dot{c}\left(\frac{s}{\mu}\right)=\frac{1}{H_{0}} X_{\frac{1}{2} H_{0}^{2}}\left(c\left(\frac{s}{\mu}\right)\right) \\
& =\frac{1}{H_{0}} X_{\frac{1}{2} H_{0}^{2}}\left(c_{0}(s)\right)=\frac{1}{H_{0}} H_{0} \cdot X_{H_{0}}\left(c_{0}(s)\right) \\
& =X_{H_{0}}\left(c_{0}(s)\right) .
\end{aligned}
$$

Portanto, $\left(\gamma_{0}(t), Y\left(\gamma_{0}(t)\right)\right)$ é uma curva integral de $X_{H_{0}}$.

Como estamos assumindo que todas as geodésicas de g tem período comum $r=2 \pi$, se $\psi_{t}^{H_{0}}$ é o fluxo de $X_{H_{0}}$ então

$$
\psi_{2 \pi}^{H_{0}}=I d
$$

em todo $T^{*} M$. Pelo Teorema 4.27 os fluxos dos campos $X_{H_{0}}$ e $X_{H_{1}}$ comutam, assim, o fluxo de $X_{H_{\alpha}}$ é:

$$
\psi_{t}^{H_{\alpha}}=\psi_{t}^{H_{0}} \circ \psi_{\alpha t}^{H_{1}} .
$$

Pelo Teorema 4.28, $X_{H_{\alpha}}$ também não corresponde ao fluxo co-geodésico de $F_{\alpha}$ mas $X_{H_{\alpha}}$ e $X_{\frac{1}{2} H_{\alpha}^{2}}$ são proporcionais

$$
X_{\frac{1}{2} H_{\alpha}^{2}}=H_{\alpha} \cdot X_{H_{\alpha}} .
$$

Assim, as curvas integrais desses campos são reparametrizações uma da outra. Análogo ao Lema 5.6, se $(\gamma(t), Y(\gamma(t)))$ é uma curva integral de $X_{\frac{1}{2} H_{\alpha}^{2}}$, então, $\left(\gamma_{0}(t), Y\left(\gamma_{0}(t)\right)\right)$ é uma curva integral de $X_{H_{\alpha}}$ onde $\gamma_{0}$ é uma reparametrização de $\gamma$ tal que $F_{\alpha}\left(\dot{\gamma}_{0}(s)\right)=1$. Para examinarmos as geodésicas fechadas de $F_{\alpha}$ tome $x \in T^{*} M$ tal que

$$
\psi_{T}^{H_{\alpha}} x=x
$$

onde T é o comprimento da geodésica na métrica $F_{\alpha}$.

Lema 5.7. A aplicação $\psi_{\alpha n T}^{H_{1}}: M \rightarrow M$ deixa a órbita $c(t)=\psi_{-t}^{H_{0}} x$ invariante para todo $n \in \mathbb{N}$, isto é, existe uma aplicação contínua $h_{n}: \mathbb{R} \rightarrow \mathbb{R}$ tal que

$$
\psi_{\alpha n T}^{H_{1}} c(t)=c\left(h_{n}(t)\right)
$$

para todo $n \in \mathbb{N}$.

Demonstração. Das equações (5.6) e (5.8) temos

$$
\psi_{T}^{H_{0}} \circ \psi_{\alpha T}^{H_{1}} x=x \Longrightarrow \psi_{-T}^{H_{0}}=\psi_{\alpha T}^{H_{1}} .
$$

Como o fluxo de $H_{0}$ e $H_{1}$ comutam e por (5.9) segue que $\psi_{\alpha T}^{H_{1}}$ deixa a órbita

$$
c(t)=\psi_{-t}^{H_{0}} x
$$

invariante, uma vez que

$$
\begin{aligned}
\psi_{\alpha T}^{H_{1}} c(t) & =\psi_{\alpha T}^{H_{1}} \circ \psi_{-t}^{H_{0}} x \\
& =\psi_{-t}^{H_{0}} \circ \psi_{\alpha T}^{H_{1}} x \\
& =\psi_{-t}^{H_{0}} \circ \psi_{-T}^{H_{0}} x \\
& =\psi_{-(t+T)}^{H_{0}} x \\
& =c(t+T)
\end{aligned}
$$


Então, $\psi_{\alpha n T}^{H_{1}}$ deixa $c$ invariante para $n=1,2, \ldots$, isto é,

$$
\psi_{\alpha n T}^{H_{1}} c(t)=c(t+n T) \quad \text { para todo } n \in \mathbb{N} .
$$

Lema 5.8. Se $\alpha T / 2 \pi$ é irracional então $c(t)$ é invariante sob $\psi_{t}^{H_{1}}$ para todo $t \in \mathbb{R}$. Se $\alpha T / 2 \pi$ é racional então $\alpha$ é racional.

Demonstração. Se $\alpha T / 2 \pi$ é irracional, então, $\alpha n T / 2 \pi$ é denso em $\mathbb{R} / \mathbb{Z} \simeq[0,1)$ e isto implica que $\alpha n T$ é denso em $[0,2 \pi)$. Como para todo $(p, \xi) \in T^{*} M$ temos

$$
\psi_{2 \pi}^{H_{1}}(p, \xi)=\left(\phi_{2 \pi}(p),\left(D \phi_{2 \pi}(p)\right)^{-1^{*}} \xi\right)=\left(p, \xi \circ\left(D \phi_{2 \pi}(p)\right)^{-1}\right)=(p, \xi \circ I d)=(p, \xi)
$$

segue que $\psi_{2 \pi}^{H_{1}}=I d$. Então, se $t \in[0,2 \pi)$ e $\left(\alpha n_{m} T\right)$ é uma subsequência que converge para $t$, como $\psi_{t}^{H_{1}}$ é contínuo em todo $T^{*} M$ segue que

$$
\psi_{t}^{H_{1}} c(s)=\lim _{n_{m} \rightarrow \infty} \psi_{\alpha n_{m} T}^{H_{1}} c(s)=\lim _{n_{m} \rightarrow \infty} c\left(s+n_{m} T\right)=c\left(s+\frac{t}{\alpha}\right) .
$$

Portanto, como $\psi_{t}^{H_{1}}$ é periódico de período $2 \pi$ segue que $c$ é invariante sob todo grupo a um parâmetro $\psi_{t}^{H_{1}}$, $t \in \mathbb{R}$. Agora, se $\alpha T / 2 \pi=m / n$, onde $m, n \in \mathbb{Z}$ e $n \neq 0$ então

$$
x=\psi_{2 \pi m}^{H_{1}} x=\psi_{\alpha n T}^{H_{1}} x=\psi_{-n T}^{H_{0}} x .
$$

Como $\psi_{t}^{H_{0}}$ tem período $2 \pi$ segue que $n T=2 \pi q$ para algum $q \in \mathbb{Z}^{*}$ e isto implica que $\alpha=m / q \in \mathbb{Q}$.

Como as projeções de $\psi_{t}^{H_{0}}$ e $\psi_{t}^{H_{\alpha}}$ sobre $\mathrm{M}$ são, a menos de reparametrização, as geodésicas de g e $F_{\alpha}$, respectivamente, temos:

Teorema 5.9. Suponha que $\alpha$ é suficientemente pequeno e irracional. Então, $\gamma$ é uma geodésica fechada de $F_{\alpha}$ (a menos de reparametrização) se e somente se $\gamma$ é uma geodésica fechada de g (a menos de reparametrização) invariante sob o grupo a um parâmetro $\phi_{t}$.

Demonstração. Assumindo que $\alpha$ é irracional e tomando uma órbita periódica de $\psi_{t}^{H_{\alpha}}$, isto é,

$$
\psi_{T}^{H_{\alpha}}(p, \xi)=(p, \xi)
$$

para algum $T \in \mathbb{R}$ e $(p, \xi) \in T^{*} M$ segue que $\frac{\alpha T}{2 \pi}$ é irracional pois se $\frac{\alpha T}{2 \pi}$ for racional obtemos, pelo Lema 5.8 , que $\alpha$ é racional e chegamos em uma contradição.

Assim, se $c(t)=\psi_{-t}^{H_{0}}(p, \xi)=(\gamma(t), g(\dot{\gamma}(t), \cdot))$, onde $\gamma$ é uma geodésica de $\mathrm{g}, \gamma(0)=p$ e $\xi=g(\dot{\gamma}(0), \cdot)$, então, $c$ é invariante sob o grupo a um parâmetro $\psi_{t}^{H_{1}}$, ou seja, $c$ é uma órbita periódica de $\psi_{t}^{H_{\alpha}}$ e $\gamma$ é, a menos de reparametrização, uma geodésica fechada de $F_{\alpha}$. Além disso, pela equação (5.10) temos

$$
\begin{aligned}
\left(\gamma\left(\frac{t}{\alpha}+s\right), g\left(\dot{\gamma}\left(\frac{t}{\alpha}+s\right), \cdot\right)\right) & =c\left(\frac{t}{\alpha}+s\right) \\
& =\psi_{t}^{H_{1}} c(s) \\
& =\psi_{t}^{H_{1}}(\gamma(s), g(\dot{\gamma}(s), \cdot)) \\
& =\left(\phi_{t}(\gamma(s)), g(\dot{\gamma}(s), \cdot) \circ\left(D \phi_{t}(p)\right)^{-1}\right)
\end{aligned}
$$

Logo, $\phi_{t}(\gamma(s))=\gamma\left(\frac{t}{\alpha}+s\right)$ para todo $t \in \mathbb{R}$, ou seja, $\gamma$ é invariante sob o grupo a um parâmetro $\phi_{t}$. Portanto, as geodésicas fechadas de $F_{\alpha}$ são, a menos de reparametrização, as geodésicas fechadas de g que são invariantes sob $\phi_{t}$. 
Por outro lado, se $\alpha$ é irracional e $\gamma(s)$ é uma geodésica de g que é invariante sob o grupo a 1-parâmetro $\phi_{t}$ temos $\phi_{t}(\gamma(s))=\gamma(h(t, s))$, para alguma função real contínua $h$. Assim,

$$
D \phi_{t}(\gamma(s)) \cdot \dot{\gamma}(s)=\frac{d}{d s} \gamma(h(t, s)) \Longrightarrow \dot{\gamma}(s)=\left(D \phi_{t}(\gamma(s))\right)^{-1} \cdot \frac{d}{d s}(\gamma(h(t, s))) .
$$

Como $\phi_{t}$ é um grupo a 1-parâmetro de isometrias então para todo $v \in T_{\gamma(h(t, s))} M$ temos

$$
\begin{aligned}
g(\dot{\gamma}(s), \cdot) \circ\left(D \phi_{t}(\gamma(s))\right)^{-1}(v) & =g\left(\dot{\gamma}(s),\left(D \phi_{t}(\gamma(s))\right)^{-1}(v)\right) \\
& =g\left(\left(D \phi_{t}(\gamma(s))\right)^{-1} \cdot \frac{d}{d s}(\gamma(h(t, s))),\left(D \phi_{t}(\gamma(s))\right)^{-1}(v)\right) \\
& =g\left(\frac{d}{d s}(\gamma(h(t, s))), v\right)
\end{aligned}
$$

Logo,

$$
g(\dot{\gamma}(s), \cdot) \circ\left(D \phi_{t}(\gamma(s))\right)^{-1}=g\left(\frac{d}{d s}(\gamma(h(t, s))), \cdot\right) .
$$

Assim, se $c(s)=(\gamma(s), g(\dot{\gamma}(s), \cdot))=\psi_{s}^{H_{0}} x$ então para todos $t, s \in \mathbb{R}$ segue que

$$
\begin{aligned}
\psi_{t}^{H_{1}} c(s) & =\psi_{t}^{H_{1}}(\gamma(s), g(\dot{\gamma}(s), \cdot)) \\
& =\left(\phi_{t}(\gamma(s)), g(\dot{\gamma}(s), \cdot) \circ\left(D \phi_{t}(\gamma(s))\right)^{-1}\right) \\
& =\left(\gamma(h(t, s)), g\left(\frac{d}{d s}(\gamma(h(t, s))), \cdot\right)\right) \\
& =c(h(t, s))
\end{aligned}
$$

Logo, $\psi_{t}^{H_{1}}$ deixa a órbita $c$ invariante para todo $t \in \mathbb{R}$. Além disso, como h é contínua, existe $T \in \mathbb{R}$ tal que $h(\alpha T, T)=2 \pi$ implicando que

$$
\psi_{T}^{H_{\alpha}} c(0)=\psi_{T}^{H_{0}} \circ \psi_{\alpha T}^{H_{1}} c(0)=\psi_{\alpha T}^{H_{1}} \circ \psi_{T}^{H_{0}} c(0)=\psi_{\alpha T}^{H_{1}} c(T)=c(h(\alpha T, T))=c(2 \pi)=c(0) .
$$

Portanto, como a projeção de $\psi_{t}^{H_{\alpha}}$ sobre M são, a menos de reparametrização, as geodésicas de $F_{\alpha}$ concluímos que as geodésicas fechadas de g que são invariantes sob $\phi_{t}$ são, a menos de reparametrização, as geodésicas fechadas de $F_{\alpha}$.

Corolário 5.10. Sejam $M=S^{2}$ com a métrica canônica de curvatura constante 1 e $\phi_{t}$ o grupo a um parâmetro de rotações deixando os polos norte e sul fixos. Então, o equador é o único grande círculo invariante sob $\phi_{t}$ e consequentemente, $F_{\alpha}$ é uma métrica Finsler em $S^{2}$, que para $\alpha$ pequeno e irracional, tem somente duas geodésicas fechadas (ver figura 5.1).

Observação 5.11. Para uma prova alternativa de que geodésicas invariantes sob $\phi_{t}$ são fechadas e outras observações sobre os exemplos de Katok em esferas e métricas Finsler Randers vide [Rob07].

\subsection{Contando geodésicas fechadas}

Com a métrica de curvatura constante $1 \mathrm{em} S^{n}$ e as métricas canônicas em $\mathbb{C} P^{n}$ e $\mathbb{H} P^{n}$ todas as geodésicas são fechadas de comprimento $2 \pi$ (a menos de reparametrização). Então, para essas variedades podemos aplicar todos os resultados obtidos na Seção 5.1. Com isso em mente, nessa seção, estimaremos no Teorema 5.15 e no Teorema 5.25 a quantidade de geodésicas fechadas de período (próximo de) $2 \pi$ em métricas Finsler definidas em $S^{n}, \mathbb{C} P^{n}$ e $\mathbb{H} P^{n}$. Mas antes disso precisamos de alguns resultados prévios.

Definição 5.12. Um plano de rotação para uma rotação particular é o plano, passando pela origem, que é mapeado nele mesmo pela rotação. 


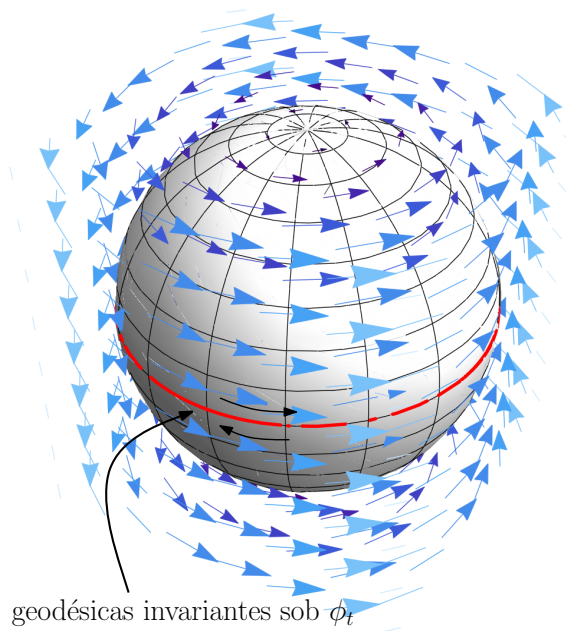

Figura 5.1: Exemplo de Katok em $S^{2}$

Lema 5.13. Seja

$$
\phi_{t}=\operatorname{diag}\left(R\left(\alpha_{1} t\right), \cdots, R\left(\alpha_{n} t\right)\right)
$$

onde $\alpha_{i}$ é um inteiro positivo e $R(\theta)$ é a rotação em $\mathbb{R}^{2}$ com ângulo $\theta$. Se os $\alpha_{i}$ 's são dois a dois distintos, então, somente os planos de rotação são invariantes sob $\phi_{t}$.

Demonstração. Seja $v=\left(v_{1}, v_{2}, \ldots, v_{2 n-1}, v_{2 n}\right) \in \mathbb{R}^{2 n}$. Como $\phi_{t} v$ tem a forma

$$
\left[\begin{array}{ccccc}
\cos \left(t \alpha_{1}\right) & -\sin \left(t \alpha_{1}\right) & & & \\
\sin \left(t \alpha_{1}\right) & \cos \left(t \alpha_{1}\right) & & & \\
& & \ddots & & \\
& & & \cos \left(t \alpha_{n}\right) & -\sin \left(t \alpha_{n}\right) \\
& & & \sin \left(t \alpha_{n}\right) & \cos \left(t \alpha_{n}\right)
\end{array}\right]\left[\begin{array}{c}
v_{1} \\
v_{2} \\
\vdots \\
v_{2 n-1} \\
v_{2 n}
\end{array}\right]=\left[\begin{array}{c}
v_{1} \cos \left(t \alpha_{1}\right)-v_{2} \sin \left(t \alpha_{1}\right) \\
v_{1} \sin \left(t \alpha_{1}\right)+v_{2} \cos \left(t \alpha_{1}\right) \\
\vdots \\
v_{2 n-1} \cos \left(t \alpha_{n}\right)-v_{2 n} \sin \left(t \alpha_{n}\right) \\
v_{2 n-1} \sin \left(t \alpha_{n}\right)+v_{2 n} \cos \left(t \alpha_{n}\right)
\end{array}\right]
$$

vemos que os planos $p_{1}=\operatorname{span}\{(1,0, \ldots, 0,0),(0,1, \ldots, 0,0)\}, \cdots, p_{n}=\operatorname{span}\{(0,0, \ldots, 1,0),(0,0, \ldots, 0,1)\}$ são invariantes sob $\phi_{t}$ pois qualquer vetor $v \in p_{i}$ temos

$$
\phi_{t} v=\left(0,0, \ldots, v_{2 i-1} \cos \left(t \alpha_{i}\right)-v_{2 i} \sin \left(t \alpha_{i}\right), v_{2 i-1} \sin \left(t \alpha_{i}\right)+v_{2 i} \cos \left(t \alpha_{i}\right), \ldots, 0,0\right) .
$$

Logo, $\phi_{t} v \in p_{i}$ para todo $t \in \mathbb{R}$. Suponha agora que existe outro plano $p \subset \mathbb{R}^{2 n}$, além dos planos $p_{i}$ 's, que é invariante sob $\phi_{t}$. Então, existe $u=\left(u_{1}, u_{2}, \ldots, u_{2 n-1}, u_{2 n}\right) \in p$ e índices $1 \leq i<j \leq n$ tais que

$$
u_{2 i-1}^{2}+u_{2 i}^{2} \neq 0 \text { e } u_{2 j-1}^{2}+u_{2 j}^{2} \neq 0 .
$$

Além disso, existe $\bar{t} \in \mathbb{R}$ tal que $\bar{t} \alpha_{i}=\pi$. Como os $\alpha_{i}$ 's são dois a dois distintos e podemos supor, sem perda de generalidade, que $\alpha_{i}>\alpha_{j}>0$ temos $0<\bar{t} \alpha_{j}=\frac{\pi}{\alpha_{i}} \alpha_{j}<\pi$. Logo,

$$
\phi_{\bar{t}} u=\left(\bar{u}_{1}, \bar{u}_{2}, \ldots,-u_{2 i-1},-u_{2 i}, \ldots, \bar{u}_{2 j-1}, \bar{u}_{2 j}, \ldots, \bar{u}_{2 n-1}, \bar{u}_{2 n}\right)
$$

onde para $1 \leq h \leq n$ temos

$$
\begin{aligned}
\bar{u}_{2 h-1} & =u_{2 h-1} \cos \left(\bar{t} \alpha_{h}\right)-u_{2 h} \sin \left(\bar{t} \alpha_{h}\right) \\
\bar{u}_{2 h} & =u_{2 h-1} \sin \left(\bar{t} \alpha_{h}\right)+u_{2 h} \cos \left(\bar{t} \alpha_{h}\right)
\end{aligned}
$$

e em particular $\left(\bar{u}_{2 j-1}, \bar{u}_{2 j}\right) \neq-\left(u_{2 j-1}, u_{2 j}\right)$. Como $p$ é invariante sob $\phi_{t}$ segue que $\phi_{\bar{t}} u \in p$ e $w=u+\phi_{\bar{t}} u \in p$, ou seja,

$$
w=\left(\widetilde{u}_{1}, \widetilde{u}_{2}, \ldots, 0,0, \ldots, \widetilde{u}_{2 j-1}, \widetilde{u}_{2 j}, \ldots, \widetilde{u}_{2 n-1}, \widetilde{u}_{2 n}\right) \in p,
$$


onde $\widetilde{u}_{h}=u_{h}+\bar{u}_{h}$ e $\left(\widetilde{u}_{2 j-1}, \widetilde{u}_{2 j}\right) \neq(0,0)$. Além disso, o vetor

$$
\phi_{t} w=\left(w_{1}, w_{2}, \ldots, 0,0, \ldots, w_{2 j-1}, w_{2 j}, \ldots, w_{2 n-1}, w_{2 n}\right) \in p
$$

onde se $h \neq i$ e $1 \leq h \leq n$ temos

$$
\begin{aligned}
w_{2 h-1} & =\widetilde{u}_{2 h-1} \cos \left(t \alpha_{h}\right)-\widetilde{u}_{2 h} \sin \left(t \alpha_{h}\right) \\
w_{2 h} & =\widetilde{u}_{2 h-1} \sin \left(t \alpha_{h}\right)+\widetilde{u}_{2 h} \cos \left(t \alpha_{h}\right) .
\end{aligned}
$$

Como $\left(\widetilde{u}_{2 j-1}, \widetilde{u}_{2 j}\right) \neq(0,0)$, para $t>0$ pequeno $\left(w_{2 j-1}, w_{2 j}\right) \neq(0,0)$ e nesse caso os vetores $w$ e $\phi_{t} w$ são linearmente independentes. Logo, $\operatorname{span}\left\{w, \phi_{t} w\right\}=p$ e qualquer vetor desse plano tem as coordenadas $2 i-1$ e $2 i$ nulas, ou seja, $u_{2 i-1}^{2}+u_{2 i}^{2}=0$. Contradição.

Portanto, somente os planos de rotação são invariantes sob $\phi_{t}$.

Lema 5.14. Sejam $\mathbb{K}=\mathbb{C}$ ou $\mathbb{K}=\mathbb{H} e$

$$
\phi_{t}=\operatorname{diag}\left(e^{i t \alpha_{1}}, \cdots, e^{i t \alpha_{n+1}}\right),
$$

onde $\alpha_{i}$ é um inteiro positivo e e $e^{i t \alpha_{j}}=\cos \left(t \alpha_{j}\right)+i \sin \left(t \alpha_{j}\right) \in \mathbb{C}$. Se os $\alpha_{j}$ 's são dois a dois distintos, então, os planos de $\mathbb{K}^{n+1}$ que são invariantes sob $\phi_{t}$ são aqueles gerados por dois vetores coordenados quaisquer.

Demonstração. Primeiro vamos mostrar que os planos gerados por dois vetores coordenados são invariantes sob $\phi_{t}$. De fato, se $\left\{v_{1}, \cdots, v_{n}\right\}$ é a base canônica de $\mathbb{K}^{n}, p_{j k}$ o plano gerado por $v_{j}, v_{k}$ e $w=v_{j} \lambda_{j}+v_{k} \lambda_{k}$, para escalares $\lambda_{j}, \lambda_{k} \in \mathbb{K}, 1 \leq j<k \leq n$, temos

$$
\phi_{t} w=\left[\begin{array}{ccccccc}
e^{i t \alpha_{1}} & & & & & & \\
& \ddots & & & & & \\
& & e^{i t \alpha_{j}} & & & & \\
& & & \ddots & & & \\
& & & & e^{i t \alpha_{k}} & & \\
& & & & & \ddots & \\
& & & & & e^{i t \alpha_{n+1}}
\end{array}\right]\left[\begin{array}{c}
0 \\
\vdots \\
\lambda_{j} \\
\vdots \\
\lambda_{k} \\
\vdots \\
0
\end{array}\right]=\left[\begin{array}{c}
0 \\
\vdots \\
e^{i t \alpha_{j}} \lambda_{j} \\
\vdots \\
e^{i t} \alpha_{k} \lambda_{k} \\
\vdots \\
0
\end{array}\right]
$$

ou seja, $\phi_{t} w=v_{j} e^{i t \alpha_{j}} \lambda_{j}+v_{k} e^{i t \alpha_{k}} \lambda_{k} \in p_{j k}$ para todo $t \in \mathbb{R}$ e $1 \leq j<k \leq n+1$.

Por um argumento análogo ao usado no Lema 5.13 segue que qualquer outro plano diferente dos planos $p_{j k}$ 's não são invariantes sob $\phi_{t}$. Portanto, segue o desejado.

Teorema 5.15. Existem métricas Finsler em $S^{2 n}$ e $S^{2 n-1}$ com somente $2 n$ geodésicas fechadas e em $\mathbb{C} P^{n} e$ $\mathbb{H} P^{n}$ com somente $n(n+1)$ e $2 n(n+1)$ geodésicas fechadas, respectivamente.

Demonstração. PARTE I: Como consequência de ([Aud03], pág 56) segue que qualquer grupo a um parâmetro fechado de isometrias em $S^{2 n-1}$ e $S^{2 n}$ é conjugado, respectivamente, a uma matriz diagonal

$$
\phi_{t}=\operatorname{diag}\left(R\left(\alpha_{1} t\right), \cdots, R\left(\alpha_{n} t\right)\right) \text { e } \phi_{t}=\operatorname{diag}\left(R\left(\alpha_{1} t\right), \cdots, R\left(\alpha_{n} t\right), 1\right)
$$

onde $\alpha_{i}$ é um inteiro positivo e $R(\theta)$ é a rotação em $\mathbb{R}^{2}$ com ângulo $\theta$.

Como as geodésicas fechadas de $S^{n}$, com a métrica Riemanniana $g$ de curvatura constante 1 , são dadas pela interseção de planos (bidimensionais) $\operatorname{com} S^{n}$ segue que as geodésicas fechadas em $S^{n}$ invariantes sob $\phi_{t}$ são dadas pela interseção de planos invariantes sob $\phi_{t}$ em $\mathbb{R}^{n+1} \operatorname{com} S^{n}$.

Se os $\alpha_{i}$ 's são dois a dois distintos, pelo Lema 5.13 somente os planos de rotação são invariantes sob $\phi_{t}$ e cada uma das geodésicas de $g$ invariantes sob $\phi_{t}$ dá origem, pelo Teorema 5.9, a duas geodésicas de $F_{\alpha}$ se $\alpha$ é irracional. Portanto, como existem $n$ planos de rotação em $\mathbb{R}^{2 n}$ e $\mathbb{R}^{2 n+1}$ segue que $S^{2 n-1}$ e $S^{2 n}$ tem $2 n$ geodésicas fechadas na métrica $F_{\alpha}$ se $\alpha$ é irracional. 
PARTE II: Para o espaço projetivo $\mathbb{C} P^{n}$ vimos em (2.4) que o grupo de isometrias desse espaço é $P U(n+1, \mathbb{C})$. Se tomarmos a fibração de Hopf

$$
S^{1} \rightarrow S^{2 n+1} \rightarrow \mathbb{C} P^{n}
$$

então $U(n+1)$ age em $S^{2 n+1} \subset \mathbb{C}^{n+1}$ de forma que somente o centro de $U(n+1)$ induz uma aplicação trivial em $\mathbb{C} P^{n}$. Como toda matriz em $U(n+1)$ é diagonalizável, então todo grupo a 1-parâmetro fechado de $U(n+1)$ é conjugado a

$$
\phi_{t}=\operatorname{diag}\left(e^{i t \alpha_{1}}, \cdots, e^{i t \alpha_{n+1}}\right) .
$$

Seja $\pi: \mathbb{C}^{n+1}-\{0\} \rightarrow \mathbb{C} P^{n}$ como definida na Seção 2.2. Pela Proposição 2.9 segue que uma geodésica fechada $\gamma \mathrm{em} \mathbb{C} P^{n}$ é a projeção de uma geodésica fechada $\beta(s)=x \cos s+u \sin s$ de $S^{2 n+1} \subset \mathbb{C}^{n+1}$ (onde $x \in \mathbb{C}^{n+1}, \gamma(0)=\pi(x)$ e $\left.\dot{\gamma}(0)=\pi(x, u)\right)$, isto é, $\gamma(s)=\pi(\beta(s))$. Suponha que $\gamma(s)$ é invariante sob $\phi_{t}$ então

$$
\phi_{t} \gamma(s)=\gamma\left(h_{t}(s)\right)
$$

para alguma função $h_{t}: \mathbb{R} \rightarrow \mathbb{R}$ e para todo $s \in \mathbb{R}$. Como

$$
\lambda \phi_{t}(\beta(s))=\phi_{t}(\lambda \beta(s)) \quad \forall \lambda \in \mathbb{C}^{*} \text { e } s \in \mathbb{R}
$$

temos pelas equações (5.13) e (5.12)

$$
\pi\left(\phi_{t}(\beta(s))\right)=\phi_{t}(\pi(\beta(s)))=\pi\left(\beta\left(h_{t}(s)\right)\right) \Longrightarrow \phi_{t}(\beta(s))=\mu_{s} \beta\left(h_{t}(s)\right)
$$

para algum $\mu_{s} \in \mathbb{C}^{*}$ e para todo $s \in \mathbb{R}$. Note que para todo $s \in \mathbb{R}, \beta(s)$ pertence a um plano $P$ gerado por $x$ e $u$. Além disso, como $\beta(0)=x, \beta(\pi / 2)=u$ então, pela equação (5.14) segue que

$$
\phi_{t} x=\phi_{t} \beta(0)=\mu_{0} \beta\left(h_{t}(0)\right)=x \mu_{0} \cos \left(h_{t}(0)\right)+u \mu_{0} \sin \left(h_{t}(0)\right) \in P
$$

e da mesma forma

$$
\phi_{t} u=x \mu_{\frac{\pi}{2}} \cos \left(h_{t}(\pi / 2)\right)+u \mu_{\frac{\pi}{2}} \sin \left(h_{t}(\pi / 2)\right) \in P .
$$

Como $\phi_{t}$ é linear então para todo $w=\lambda_{1} x+\lambda_{2} u \in P$ temos

$$
\begin{aligned}
\phi_{t} w & =\phi_{t}\left(\lambda_{1} x+\lambda_{2} u\right) \\
& =\lambda_{1} \phi_{t} x+\lambda_{2} \phi_{t} u \\
& =\lambda_{1}\left(x \mu_{0} \cos \left(h_{t}(0)\right)+u \mu_{0} \sin \left(h_{t}(0)\right)\right)+\lambda_{2}\left(x \mu_{\frac{\pi}{2}} \cos \left(h_{t}(\pi / 2)\right)+u \mu_{\frac{\pi}{2}} \sin \left(h_{t}(\pi / 2)\right)\right) \\
& =\left(\lambda_{1} \mu_{0} \cos \left(h_{t}(0)\right)+\lambda_{2} \mu_{\frac{\pi}{2}} \cos \left(h_{t}(\pi / 2)\right)\right) x+\left(\lambda_{1} \mu_{0} \sin \left(h_{t}(0)\right)+\lambda_{2} \mu_{\frac{\pi}{2}} \sin \left(h_{t}(\pi / 2)\right)\right) u
\end{aligned}
$$

ou seja, $\phi_{t} w \in P$. Logo, o plano $P$ é invariante sob $\phi_{t}$.

Assim, toda geodésica fechada $\gamma \mathrm{em} \mathbb{C} P^{n}$ invariante sob $\phi_{t}$ é levantada em um plano de $\mathbb{C}^{n+1}$ invariante sob $\phi_{t}$ e pelo Lema 5.14 os planos invariantes sob $\phi_{t}$ são gerados por dois vetores coordenados quaisquer. Isso reduz a situação para a ação de $U(2)$ em $S^{1} \rightarrow S^{3} \rightarrow \mathbb{C} P^{1} \cong S^{2}$. Como vimos na primeira parte da demonstração, $S^{2}$ possui somente 2 geodésicas invariantes sob o grupo a 1-parâmetro de rotações. Então, como geodésicas vindas de planos distintos são distintas e existem

$$
\frac{(n+1) !}{2 !(n-1) !}=\frac{n(n+1)}{2}
$$

planos gerados por dois vetores coordenados segue que

$$
\frac{n(n+1)}{2} \cdot 2=n(n+1)
$$

é a quantidade de geodésicas fechadas em $\mathbb{C} P^{n}$ na métrica $F_{\alpha}$ se $\alpha$ é irracional.

PARTE III: Como vimos em (2.5), o grupo de isometrias para o espaço projetivo $\mathbb{H} P^{n}$ é $P U(n+1, \mathbb{H})$. 
Tomando a fibração de Hopf

$$
S^{3} \rightarrow S^{4 n+3} \rightarrow \mathbb{H} P^{n}
$$

então, $S p(n+1)$ age em $S^{4 n+3} \subset \mathbb{H} P^{n}$ e somente as matrizes $I$ e $-I$ induzem uma aplicação trivial em $\mathbb{H} P^{n}$. Como toda matriz $A \in S p(n+1)$ é unitária segue de ([Lee48], pág 259) que $A$ pode ser transformada por uma matriz unitária em uma matriz na forma diagonal, onde os elementos da diagonal são números complexos de norma 1 , ou seja, tem a forma $e^{i \theta}=\cos (\theta)+i \sin (\theta)$ para algum $\theta \in \mathbb{R}$. Logo, todo grupo a 1-parâmetro fechado de $S p(n+1)$ é conjugado a

$$
\phi_{t}=\operatorname{diag}\left(e^{i t \alpha_{1}}, \cdots, e^{i t \alpha_{n+1}}\right) .
$$

Pela Proposição 2.9, assim como para $\mathbb{C} P^{n}$, segue que as geodésicas fechadas invariantes sob $\phi_{t}$ estão contidas em planos invariantes sob $\phi_{t}$. Pelo Lema 5.14 esses planos invariantes são gerados por dois vetores coordenados quaisquer e da equação (5.15) obtemos $n(n+1) / 2$ desses planos.

Precisamos agora observar a ação de $S p(2)$ em cada um dos planos invariantes sob $\phi_{t}$, ou melhor, a ação de $S p(2) \cong S U(4)$ em $S^{3} \rightarrow S^{7} \rightarrow \mathbb{H} P^{1} \cong S^{4}$. Como $S^{4}$ possui 4 geodésicas invariantes sob o grupo a 1-parâmetro de rotações (ou seja, existem 4 geodésicas fechadas invariantes sob $\phi_{t}$ em cada um dos planos citados) e existem $n(n+1) / 2$ desses planos segue-se que existem

$$
\frac{n(n+1)}{2} \cdot 4=2 n(n+1)
$$

geodésicas fechadas em $\mathbb{H} P^{n}$ na métrica $F_{\alpha}$ se $\alpha$ é irracional.

Além da métrica canônica existe outra métrica Riemanniana definida em $S^{n}$ ([Bes12], pág 120) satisfazendo as hipóteses para a construção da métrica Finsler como na equação (5.3), isto é, uma métrica em que todas as geodésicas são fechadas de período $2 \pi$ e que admite um grupo a um parâmetro de isometrias. Também existem métricas Riemannianas em $S^{2}$ em que todas as geodésicas são fechadas e que não admitem grupos de isometrias ([Bes12], pág 126). No entanto, não existem qualquer exemplo de métricas com uma quantidade de geodésicas menor do que as que encontramos no Teorema 5.15.

Por um teorema de Bott e Samelson ([Bes12], pág 186) segue que as únicas variedades que admitem métricas Riemannianas com todas as geodésicas fechadas de mesmo período são ou difeomorfas a $\mathbb{R} P^{n}$ ou tem a mesma cohomologial integral de anéis que $S^{n}, \mathbb{C} P^{n}, \mathbb{H} P^{n}$ e $C a P^{n}$ (plano projetivo de Cayley que não abordaremos nesse trabalho). Portanto, esferas e espaços projetivos são as únicas variedades em que obtemos exemplos, como os mostrados anteriormente, de métricas Finsler com uma quantidade finita de geodésicas fechadas.

Mostraremos a seguir que o número de geodésicas fechadas nos exemplos de esferas e espaços projetivos tem algum significado topológico e são "ótimos"em algum sentido. Mas antes disso, enunciaremos sem a demonstração alguns resultados da teoria de Lusternik-Schnirelmann e da teoria de Morse necessários para a demonstração do Teorema 5.24 e do Teorema 5.25.

Definição 5.16. Seja $M$ uma variedade de dimensão $n$ e $f: M \rightarrow \mathbb{R}$. Um ponto $p \in M$ é dito crítico de $f$ se $d f(p)=0$. Um número $c \in \mathbb{R}$ é dito valor crítico de $f$ se existe algum ponto crítico $p$ de $f$ tal que $f(p)=c$. Se $c \in \mathbb{R}$ não é valor crítico então $c$ é chamado de valor regular. O ponto crítico $p$ é dito não degenerado se a Hessiana

$$
d^{2} f(p): T_{p} M \times T_{p} M \rightarrow \mathbb{R}
$$

de $f$ em $p$ dada por

$$
d^{2} f(p)=\sum_{i, j=1}^{n} \frac{\partial^{2} f}{\partial x^{i} \partial x^{j}}(p) d x^{i} \otimes d x^{j}
$$

tiver núcleo trivial. Aqui as derivadas parciais são tomadas em relação a um sistema de coordenadas $\left(x^{i}, \ldots, x^{n}\right)$ em torno de $p$. O índice de Morse de $p$ é a dimensão do subespaço maximal de $T_{p} M$ onde $d^{2} f(p)$ é negativa-definida.

Definição 5.17. Seja $f: M \rightarrow \mathbb{R}$ então $f$ é dita uma função de Morse se todos os seus pontos críticos são não-degenerados. 
Teorema 5.18 ([DFBN90], pág 195). Seja M uma variedade diferenciável fechada de dimensão $n$ e denote por $b_{k}(M)$ a dimensão do k-ésimo grupo de homologia de $M$ sob qualquer corpo (i.e, dimensão como espaço vetorial). Então, para qualquer função de Morse f em M a seguinte "desigualdade de Morse"vale:

$$
\mu_{k}(f) \geq b_{k}(M), \quad k=0,1, \ldots, n
$$

onde $\mu_{k}(f)$ é o número de pontos críticos de $f$ de índice $k$.

Observação 5.19. Os elementos $b_{k}(M)$ são conhecidos como números de Betti.

Definição 5.20. Seja $X$ um espaço topológico Hausdorff e $A \subset X$ um conjunto fechado de $X$. O conjunto $A$ diz-se de categoria $k=\operatorname{cat}_{X}(A)$ com respeito ao espaço topológico $\mathrm{X}$ se $k$ é o menor número para o qual $A$ pode ser escrito como a união

$$
A=A_{1} \cup \cdots \cup A_{k}
$$

de $k$ conjuntos fechados $A_{1}, \cdots, A_{k}$, onde cada um deles é contrátil em X (a um ponto). Denotamos

$$
\text { cat }_{X} X=\operatorname{cat} X \text {. }
$$

Definição 5.21. O comprimento da cohomologia ou comprimento cup é o maior número de (não necessariamente distintas) classes de cohomologia cujo produto cup é não nulo.

Teorema 5.22 ([DFBN90], pág 226). Seja M uma variedade suave de dimensão $n$, fechada e conexa e $f: M \rightarrow \mathbb{R}$ uma função suave. O número $k$ (que pode ser infinito) de pontos críticos distintos de $f$ é limitado inferiormente por

$$
k \geq \operatorname{cat}(M) .
$$

Teorema 5.23 ([DFBN90], pág 234). Se M é uma variedade suave de dimensão ne compacta cujo comprimento da cohomologia é $\lambda$ então

$$
\operatorname{cat}(M) \geq \lambda+1 .
$$

Teorema 5.24. Seja $(M, g)$ uma variedade Riemanniana tal que todas as geodésicas de $M$ são fechadas de mesmo período $2 \pi$. Então, qualquer métrica Finsler em $M$ suficientemente próxima de g tem pelo menos $\operatorname{dim} M$ geodésicas fechadas de comprimento próximo de $2 \pi$.

Demonstração. Seja $(\mathrm{M}, \mathrm{g})$ uma variedade Riemanniana de dimensão $\mathrm{n}$ tal que todas as geodésicas são fechadas de período $2 \pi$ e seja $C=T_{1} M / S^{1}$ como definido em (1.30).

Como vimos na seção 4.6 o problema de encontrar geodésicas em uma variedade pode ser transportado para o problema de encontrar pontos críticos de uma função. Pelo Corolário 4.53 vemos que qualquer métrica Finsler $F$ suficientemente próxima da métrica Riemanniana $g$ em $\mathrm{M}$ tem tantas geodésicas fechadas de comprimento próximo de $2 \pi$ quanto uma função em $C=T_{1} M / S^{1}$ tem pontos críticos.

Por [Wei74] segue que a classe de Euler $e \in H^{2}(C)$ da fibração $T_{1} M \rightarrow C$ satisfaz $e^{n-1}=[C] \neq 0$, ou seja, o comprimento da cohomologia é pelo menos $n-1$. Logo, pelos Teoremas 5.22 e 5.23 segue que o número $k$ de pontos críticos de qualquer função real e suave em $C$ é tal que

$$
k \geq \operatorname{cat}(C) \geq(n-1)+1=n=\operatorname{dim} M .
$$

Portanto, qualquer métrica Finsler em $M$ suficientemente próxima de $g$ tem pelo menos $\operatorname{dim} M$ geodésicas fechadas de comprimento próximo de $2 \pi$.

Teorema 5.25. Seja $M=S^{n}, \mathbb{C} P^{n}, \mathbb{H} P^{n}$. Se todas as geodésicas de comprimento próximo de $2 \pi$ na métrica Riemanniana canônica $g$ definida nessas variedades são não-degeneradas então qualquer métrica Finsler suficientemente próxima de g tem pelo menos $2 n$ geodésicas em $S^{2 n}$ e $S^{2 n-1}, n(n+1)$ em $\mathbb{C} P^{n}$ e $2 n(n+1)$ em $\mathbb{H} P^{n}$ de comprimento próximo de $2 \pi$. 
Demonstração. Sejam M como no enunciado e $g$ a métrica Riemanniana canônica definida em M. Como vimos no Corolário 4.53, qualquer métrica Finsler $F$ em M suficientemente próxima de $g$ tem pelo menos tantas geodésicas fechadas de comprimento próximo de $2 \pi$ quanto uma função $\varphi$ em $\mathrm{C}$ tem pontos críticos. Se todas as geodésicas de $F$ com comprimento próximo de $2 \pi$ são não degeneradas então pelo Teorema 5.18 segue que o número $k$ de pontos críticos de $\varphi$ é tal que

$$
k=\sum_{i} \mu_{i}(\varphi) \geq \sum_{i=0}^{\operatorname{dim} C} b_{i}(C) .
$$

Como vimos na seção 3.7, usando a sequência de Gysin na fibração $S^{n-1} \rightarrow T_{1} M \rightarrow M$ encontramos a cohomologia de $T_{1} M$ e usando a sequência de Gysin em $S^{1} \rightarrow T_{1} M \rightarrow C$ podemos encontrar os números de Betti de $C$. Assim:

(i) Pelo Lema 3.41, se $M=S^{2 n}$ temos $b_{i}(C)=1$ se $i$ é par e $0 \leq i \leq 4 n-2$. Logo,

$$
\sum_{i=0}^{4 n-2} b_{i}(C)=\frac{4 n-2}{2}+1=2 n
$$

(ii) Pelo Lema 3.42, se $M=S^{2 n-1}$ temos $b_{i}(C)=1$ se $i$ é par e $0 \leq i \leq 4 n-4$ exceto $b_{2 n-2}(C)=2$. Logo,

$$
\sum_{i=0}^{4 n-4} b_{i}(C)=\frac{2 n-4}{2}+1+2+\frac{(4 n-4)-2 n}{2}+1=2 n .
$$

(iii) Pelo Lema 3.43, se $M=\mathbb{C} P^{n}$ temos

$$
b_{0}(C)=1, b_{2}(C)=2, b_{4}(C)=3, \ldots, b_{2 n-2}(C)=n=b_{2 n}(C), b_{2 n+1}(C)=n-1, \ldots, b_{4 n-2}(C)=1 .
$$

Logo,

$$
\sum_{i=0}^{4 n-2} b_{i}(C)=\frac{(1+n) n}{2} \cdot 2=n(n+1) .
$$

(iv) Pelo Lema 3.44, se $M=\mathbb{H} P^{n}$ temos

$b_{0}(C)=b_{2}(C)=1, b_{4}(C)=b_{6}(C)=2, \ldots, b_{4 n-4}(C)=b_{4 n-2}(C)=n=b_{4 n}(C)=b_{4 n+2}(C), \ldots, b_{8 n-2}(C)=1$.

Logo,

$$
\sum_{i=0}^{8 n-2} b_{i}(C)=\frac{(1+n) n}{2} \cdot 4=2 n(n+1)
$$

Portanto, toda métrica Finsler suficientemente próxima de $g$ tem pelo menos $2 n$ geodésicas em $S^{2 n}$ e $S^{2 n-1}, n(n+1)$ em $\mathbb{C} P^{n}$ e $2 n(n+1)$ em $\mathbb{H} P^{n}$ de comprimento próximo de $2 \pi$.

Assim, comparando o Teorema 5.15 e o Teorema 5.25 vemos que entre todas as métricas Finsler suficientemente próximas da métrica Riemanniana canônica, somente os exemplos de $F_{\alpha}$ (construídas na demonstração do Teorema 5.15) em $S^{2 n}$ são ótimos, no sentido de que $F_{\alpha}$ possui a menor quantidade de geodésicas fechadas estimada no Teorema 5.25. Mas todos os exemplos são ótimos se considerarmos somente métricas Finsler com geodésicas fechadas não degeneradas. 


\section{Capítulo 6}

\section{Propriedades Geométricas dos Exemplos de Katok}

Nesse capítulo vamos examinar algumas propriedade da co-métrica Finsler

$$
H_{\alpha}=H_{0}+\alpha H_{1}
$$

em $M=S^{n}, \mathbb{C} P^{n}, \mathbb{H} P^{n}$ onde $H_{0}$ e $H_{1}$ são como em (4.9) e (4.5), respectivamente. Na maioria dos casos faremos os cálculos somente para $M=S^{2}$, os outros são similares.

\subsection{Comprimento das Geodésicas}

Teorema 6.1. O comprimento das geodésicas fechadas de $F_{\alpha}$ em $S^{2}$ são iguais a $2 \pi /(1+\alpha)$ e $2 \pi /(1-\alpha)$ dependendo se a geodésica fechada está a favor ou na direção oposta à direção da rotação.

Demonstração. Vimos anteriormente no capítulo 5 que se $\left(\gamma(t), Y(\gamma(t))\right.$ é uma curva integral de $X_{\frac{1}{2} H_{\alpha}^{2}}$, então, $\left(\gamma_{0}(t), Y\left(\gamma_{0}(t)\right)\right.$ é uma curva integral de $X_{H_{\alpha}}$, onde $\gamma_{0}$ é uma reparametrização de $\gamma$ tal que $F_{\alpha}\left(\dot{\gamma}_{0}(s)\right)=$ 1. Assim, o comprimento de uma geodésica na métrica Finsler $F_{\alpha}$ concorda com o período das órbitas de $X_{H_{\alpha}}$. Seja

$$
c(t)=\psi_{t}^{H_{0}} x
$$

uma geodésica fechada de $H_{0}$, em $T^{*} M$, invariante sob $\phi_{t}$. Pelo Corolário 5.10, para $M=S^{2}$, as únicas geodésicas invariantes sob o grupo a 1-parâmetro de rotações

$$
\phi_{t}=\left[\begin{array}{ccc}
\cos t & -\sin t & 0 \\
\sin t & \cos t & 0 \\
0 & 0 & 1
\end{array}\right]
$$

são $\gamma_{1}(t)=(\cos t, \sin t, 0)$ e $\gamma_{2}(t)=(\cos t,-\sin t, 0)$, isto é, o equador percorrido na direção anti-horária e horária, respectivamente. Veja que

$$
\begin{aligned}
\phi_{s}\left(\gamma_{1}(t)\right) & =\left[\begin{array}{ccc}
\cos s & -\sin s & 0 \\
\sin s & \cos s & 0 \\
0 & 0 & 1
\end{array}\right]\left[\begin{array}{c}
\cos t \\
\sin t \\
0
\end{array}\right]=\left[\begin{array}{c}
\cos s \cos t-\sin s \sin t \\
\sin s \cos t+\cos s \sin t \\
0
\end{array}\right] \\
& =\left[\begin{array}{c}
\cos (t+s) \\
\sin (t+s) \\
0
\end{array}\right]=\gamma_{1}(t+s)
\end{aligned}
$$


$\mathrm{e}$

$$
\begin{aligned}
\phi_{s}\left(\gamma_{2}(t)\right) & =\left[\begin{array}{ccc}
\cos s & -\sin s & 0 \\
\sin s & \cos s & 0 \\
0 & 0 & 1
\end{array}\right]\left[\begin{array}{c}
\cos t \\
-\sin t \\
0
\end{array}\right]=\left[\begin{array}{c}
\cos s \cos t+\sin s \sin t \\
\sin s \cos t-\cos s \sin t \\
0
\end{array}\right] \\
& =\left[\begin{array}{c}
\cos (t-s) \\
-\sin (t-s) \\
0
\end{array}\right]=\gamma_{2}(t-s)
\end{aligned}
$$

Como vimos na demonstração do Teorema 5.9, se $\gamma(t)$ é uma geodésica de $g$ invariante sob $\phi_{s}$ e tal que $\phi_{s}(\gamma(t))=\gamma(h(t, s))$ para alguma função real contínua h então se $c(t)=\psi_{t}^{H_{0}} x$ temos

$$
\psi_{s}^{H_{1}} c(t)=c(h(t, s)) .
$$

Logo, como no nosso caso $h(t, s)=t+s$ e $h(t, s)=t-s$ temos

$$
\psi_{s}^{H_{1}} c(t)=c(t \pm s) .
$$

Assim,

$$
\psi_{t}^{H_{\alpha}} x=\psi_{t}^{H_{0}} \circ \psi_{\alpha t}^{H_{1}} x=\psi_{\alpha t}^{H_{1}} \circ \psi_{t}^{H_{0}} x=\psi_{\alpha t}^{H_{1}} c(t)=c(t \pm \alpha t) .
$$

Como $c$ tem período $2 \pi$ então se $T$ é o período de $\psi_{t}^{H_{\alpha}} x$ temos

$$
T(1 \pm \alpha)=2 \pi \Longrightarrow T=\frac{2 \pi}{1 \pm \alpha}
$$

Portanto, os comprimentos das geodésicas $\gamma_{1}$ e $\gamma_{2}$ na métrica $F_{\alpha}$ são $2 \pi /(1+\alpha)$ e $2 \pi /(1-\alpha)$, respectivamente.

Analogamente para a métrica $F_{\alpha}$ em $S^{n}$ com $\phi_{t}$ como definido na PARTE I do Teorema 5.15 segue que o comprimento das geodésicas são $2 \pi /\left(1+\alpha \alpha_{i}\right)$ e $2 \pi /\left(1-\alpha \alpha_{i}\right)$, respectivamente.

Teorema 6.2. Se $|\alpha|<1$ então $F_{\alpha}$ como definido em (5.3) é uma métrica Finsler.

Demonstração. Denote por $N(p, y)=\sqrt{g_{p}(y, y)}$ e $\left(g_{i j}\right)$ a matriz simétrica positiva-definida associada a métrica Riemanniana $g$. Como a aplicação $y \longmapsto g(y, \cdot)$ é uma bijeção e $H_{0}(p, \xi)=\sqrt{g^{i j}(p) \xi_{i} \xi_{j}}$, onde $\left(g^{i j}(p)\right)=\left(g_{i j}(p)\right)^{-1}$ temos

$$
H_{0}\left(p, g_{p}(y, \cdot)\right)=\sqrt{g^{i j}(p) g_{i j}(p) y^{i} g_{i j}(p) y^{j}}=\sqrt{g_{i j}(p) y^{i} y^{j}}=N(p, y)
$$

e

$$
H_{1}\left(p, g_{p}(y, \cdot)\right)=g_{p}\left(y, V_{p}\right) .
$$

Logo, $H_{0}\left(p, g_{p}(y, \cdot)\right)$ é uma norma Riemanniana associada a $g$ e $\beta:=H_{1}\left(p, g_{p}(y, \cdot)\right)$ é uma 1-forma em $T M$ que para cada $p \in M$ associa o funcional linear $g_{p}\left(V_{p}, \cdot\right)$. Pelo Teorema 1.30 segue que

$$
F(p, y):=H_{\alpha}\left(p, g_{p}(y, \cdot)\right)=\sqrt{g_{i j}(p) y^{i} y^{j}}+\alpha \beta(y)
$$

é uma métrica Finsler se, e somente se, $\|\alpha \beta\|_{g}<1$. Observe que

$$
\begin{aligned}
\|\alpha \beta\|_{g} & =|\alpha| \sup \left\{\beta(y), N(p, y)=1, y \in T_{p} M e p \in M\right\} \\
& =|\alpha| \sup \left\{g_{p}\left(y, V_{p}\right), N(p, y)=1, y \in T_{p} M e p \in M\right\}
\end{aligned}
$$

e para $y \in T_{p} M$ tal que $N(p, y)=1$ temos

$$
g_{p}\left(y, V_{p}\right)=N(p, y) N\left(p, V_{p}\right) \cos (\theta)=N\left(p, V_{p}\right) \cos (\theta)
$$


onde $\theta$ é ângulo entre $y$ e $V_{p}$. Assim, como $N\left(p, V_{p}\right) \leq 1$ para todo $p$ em $\mathrm{M}$ então $\mathrm{F}$ é uma métrica Finsler se, e somente se,

$$
1>\|\alpha \beta\|_{g}=|\alpha| \sup _{p \in M} N\left(p, V_{p}\right)=|\alpha| .
$$

Observe que derivando $\mathrm{F}$ em relação a y temos

$$
\begin{aligned}
\frac{1}{2} \frac{\partial F^{2}}{\partial y^{i}}(p, y) & =\frac{1}{2} \frac{\partial}{\partial y^{i}}\left[H_{\alpha}^{2}\left(p^{i}, g_{i j} y^{i}\right)\right] \\
& =\frac{1}{2} \frac{\partial H_{\alpha}^{2}}{\partial \xi_{i}}\left(p^{i}, g_{i j} y^{i}\right) \cdot g_{i j}
\end{aligned}
$$

e derivando novamente em relação a y temos

$$
\begin{aligned}
f_{i j}(p, y) & =\frac{1}{2} \frac{\partial^{2} F^{2}}{\partial y^{i} \partial y^{j}}(p, y) \\
& =\frac{1}{2} \frac{\partial}{\partial y^{j}}\left[\frac{\partial H_{\alpha}^{2}}{\partial \xi_{i}}\left(p^{i}, g_{i j} y^{i}\right) \cdot g_{i j}\right] \\
& =\frac{1}{2} \frac{\partial^{2} H_{\alpha}^{2}}{\partial \xi_{i} \partial \xi_{j}}\left(p, g_{p}(y, \cdot)\right) \cdot g_{i j} \cdot g_{i j}+\frac{1}{2} \frac{\partial H_{\alpha}^{2}}{\partial \xi_{i}}\left(p, g_{p}(y, \cdot)\right) \cdot \frac{\partial}{\partial y_{i}}\left(g_{i j}\right) \\
& =h_{i j}\left(p, g_{p}(y, \cdot)\right)\left(g_{i j}\right)^{2}
\end{aligned}
$$

Logo,

$$
h_{i j}=f_{i j}\left(g^{i j}\right)^{2}=f_{i j} \circ H_{0}^{2} .
$$

Então, se $|\alpha|<1$ segue-se que a forma bilinear $h_{i j}$ é positiva definida pois $f_{i j}$ nesse caso é positiva definida. Portanto, como $H_{\alpha}$ é diferenciável em $T^{*} M \backslash\{0\}$ e homogênea de grau 1 segue que $H_{\alpha}$ é uma co-norma Finsler se $|\alpha|<1$ e pelo Teorema 1.25 concluímos que $F_{\alpha}$ é uma métrica Finsler.

Observação 6.3. Veja que quando $\alpha \rightarrow 1$ o comprimento das geodésicas fechadas em $S^{2}$ (na métrica $F_{\alpha}$ ) tendem a $\pi$ e $\infty$, respectivamente.

Os exemplos de $F_{\alpha}$ também são interessantes quando $\alpha$ é racional. Seja $\alpha=k / m$ onde $k$ e $m$ são relativamente primos. As geodésicas fechadas invariantes sob $\phi_{t}$ então tem período

$$
\frac{2 \pi}{1+\alpha}=\frac{2 \pi m}{k+m}
$$

$\mathrm{e}$

$$
\frac{2 \pi}{1-\alpha}=\frac{2 \pi m}{k-m}
$$

respectivamente. Mas, afirmo que todas as outras geodésicas tem período $2 \pi m$. De fato, se $c(t)=\psi_{t}^{H_{0}} x$ é uma órbita fechada de $X_{H_{0}}$ temos

$$
\psi_{t}^{H_{\alpha}} x=\psi_{t}^{H_{0}} \circ \psi_{\alpha t}^{H_{1}} x=\psi_{\alpha t}^{H_{1}} \circ \psi_{t}^{H_{0}} x=\psi_{\alpha t}^{H_{1}} c(t)=\psi_{\frac{k t}{m}}^{H_{1}} c(t) .
$$

Como nesse caso $c(t)$ não é invariante sob $\psi_{t}^{H_{1}}$ então se $T$ é o período de $\psi_{t}^{H_{\alpha}}$ segue que $T / m=2 \pi$. Assim:

Teorema 6.4. Existem métricas Finsler em $S^{n}, \mathbb{C} P^{n}$ e $\mathbb{H} P^{n}$ com todas as geodésicas fechadas mas que não tem o mesmo período.

Por exemplo, para $S^{2} \operatorname{com} \alpha=k /(k+1)$ então os períodos das geodésicas fechadas são:

$$
\frac{2 \pi}{1+\alpha}=\frac{2 \pi(k+1)}{2 k+1}, \frac{2 \pi}{1-\alpha}=2 \pi(k+1) \text { e } 2 \pi(k+1)
$$

Após uma reparametrização, todas as geodésicas tem comprimento $2 \pi$, exceto por uma que tem comprimento $2 \pi /(2 k+1)$. 


\subsection{Aplicação de Poincaré dos Exemplos de Katok}

Seja $(M, \omega)$ uma variedade simplética e $\mathrm{H}$ um hamiltoniano com campo Hamiltoniano $X_{H}$ e fluxo $\psi_{t}$, onde $c(t)$ é uma órbita fechada de período T, isto é, $c(0)=c(T)=x$.

Definição 6.5. Seja $\Sigma^{\prime} \subset M$ uma subvariedade de codimensão 1 transversal a $X_{H}$, ou seja,

$$
T_{y} M=T_{y} \Sigma^{\prime} \oplus \operatorname{span}\left\{X_{H}(y)\right\}
$$

para todo $y \in \Sigma^{\prime}$ numa vizinhança de x. Então, $\Sigma^{\prime}$ é chamada uma seção transversal local de $c(t)$ no ponto x.

Em uma vizinhança $U \subset \Sigma^{\prime}$ de x o fluxo $\psi_{t}$ define uma aplicação suave

$$
\mathscr{P}: U \subset \Sigma^{\prime} \rightarrow \Sigma^{\prime}
$$

definido da seguinte forma. Dado $y \in U \subset \Sigma^{\prime}$, próximo de $x=c(0)$, seguimos a órbita de $y$ até $\psi_{t} y$ intersectar $\Sigma^{\prime}$ pela primeira vez, no tempo $\tau(y)>0$, e definimos

$$
\mathscr{P}(y)=\psi_{\tau(y)}(y) \in \Sigma^{\prime}, \quad y \in U \subset \Sigma^{\prime} .
$$

Como o campo de vetores $X_{H}$ é transversal à seção $\Sigma^{\prime}$, a aplicação $y \longmapsto \tau(y) \in \mathbb{R}$ é suave, definida em uma vizinhança de $\mathrm{x}$ e unicamente determinado pelas condições

$$
\left\{\begin{array}{l}
\tau(x)=T \\
\psi_{\tau(y)} \in \Sigma^{\prime}, y \in \Sigma^{\prime} \cap U
\end{array}\right.
$$

Definição 6.6. A aplicação $\mathscr{P}$ é chamada aplicação de Poincaré de $c$ e $P=d \mathscr{P}(x)$ é chamada de aplicação linearizada de Poincaré de c.

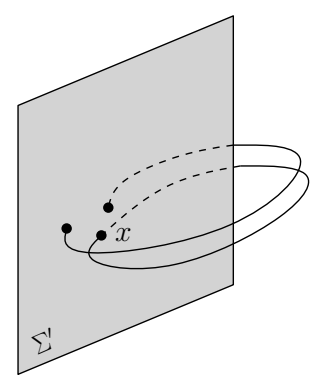

Figura 6.1: Aplicação de Poincaré

Definição 6.7. Se todos os autovalores da aplicação linearizada de Poincaré de $c$ tem norma 1 dizemos que $c$ é elíptica.

Agora suponha que $c(t)$ tenha energia $\mu$, isto é, $H(c(t))=\mu$ e que a superfície de energia

$$
S=\{x \in M ; H(x)=\mu\}
$$

é regular e, portanto, uma subvariedade de dimensão $2 n-1$. Se $\Sigma^{\prime} \subset M$ é uma seção transversal da órbita periódica $c(t)$ então como $\mathrm{H}$ é constante ao longo das órbitas de $X_{H}$ segue que a aplicação $\mathscr{P}: U \cap \Sigma^{\prime} \rightarrow \Sigma^{\prime}$ deixa a superfície de energia invariante

$$
\mathscr{P}: U \cap \Sigma^{\prime} \cap S \rightarrow \Sigma^{\prime} \cap S .
$$

Logo, podemos definir a seção transversal $\Sigma$ na superfície de energia $S$ e a restrição da aplicação de Poincaré por

$$
\Sigma:=\Sigma^{\prime} \cap S \text { e } \mathscr{P}: U \cap \Sigma \rightarrow \Sigma .
$$


Teorema 6.8. A seção transversal $\Sigma=\Sigma^{\prime} \cap S$ da órbita periódica $c(t)$ na superfície de energia $S$ é uma subvariedade simplética de $(M, \omega)$ de dimensão $2 n-2$ equipada com a forma simplética $\omega^{\prime}=i^{*} \omega$, onde $i: \Sigma \rightarrow M$ é a inclusão. A aplicação de Poincaré $\mathscr{P}: U \cap \Sigma \rightarrow \Sigma$ preserva a forma simplética, ou seja, $\mathscr{P}^{*} \omega^{\prime}=\omega^{\prime}$.

Demonstração. Primeiro mostraremos que $\omega^{\prime}$ é uma forma simplética. Como a 2-forma $\omega^{\prime}$ é fechada pois $d i^{*} \omega=i^{*} d \omega=0$, precisamos mostrar apenas que $\omega^{\prime}$ é não degenerada. Observe que o espaço tangente de $\mathrm{S}$ pode ser representado por

$$
T_{y} S=\operatorname{ker} d H(y)=\operatorname{ker} \omega\left(X_{H}, \cdot\right)=\left\{v \in T_{y} M ; \omega\left(X_{H}, v\right)=0\right\}
$$

Como $\Sigma$ em S é transversal à $X_{H}$ temos

$$
T_{y} S=T_{y} \Sigma \oplus \operatorname{span}\left\{X_{H}\right\} .
$$

Assuma que $v \in T_{y} \Sigma$ satisfaz

$$
\omega(v, u)=0 \text { para todo } u \in T_{y} \Sigma \text {. }
$$

Por (6.3) segue que todo vetor $\rho \in T_{y} S$ pode ser representado por $\rho=u+\lambda X_{H}$, onde $\lambda \in \mathbb{R}$ e $u \in T_{y} \Sigma$. Como $\Sigma \subset S$, usamos a definição de um campo de vetores Hamiltoniano e concluímos que

$$
\omega(v, \rho)=\omega(v, u)+\lambda \omega\left(v, X_{H}(y)\right)=\lambda d H(y) v=0
$$

para todo $\rho \in T_{y} S$. Assim, $v$ pertence ao subespaço 1-dimensional $\operatorname{ker}\left(\left.\omega\right|_{S}\right)=\operatorname{span}\left\{X_{H}\right\}$. Mas, como $v \in$ $T_{y} \Sigma$ e $X_{H} \notin T_{y} \Sigma$ concluímos que $v=0$. Logo, $\omega^{\prime}=i^{*} \omega$ é não degenerada. Agora queremos mostrar que $\mathscr{P}^{*} \omega^{\prime}=\omega^{\prime}$, isto é,

$$
\omega(u, v)=\omega(d \mathscr{P}(y) u, d \mathscr{P}(y) v)
$$

para todo $u, v \in T_{y} \Sigma$. Diferenciando $\mathscr{P}$ temos

$$
d \mathscr{P}(y)=\frac{d}{d y} \psi_{\tau(y)}(y)=X_{H}\left(\psi_{\tau}(y)\right) d \tau(y)+d \psi_{\tau}(y)
$$

para $\tau=\tau(y)$. Como $d \psi_{\tau}(y)$ é simplética, abreviando $l:=d \tau(y)$ e $\rho=\psi_{\tau}(y)$, segue que para todo $u, v \in T_{y} \Sigma$

$$
\begin{aligned}
\omega(u, v) & =\omega\left(d \psi_{\tau}(y) u, d \psi_{\tau}(y) v\right) \\
& =\omega\left(d \mathscr{P}(y) u-l(u) X_{H}(\rho), d \mathscr{P}(y) v-l(v) X_{H}(\rho)\right) \\
& =\omega(d \mathscr{P}(y) u, d \mathscr{P}(y) v)
\end{aligned}
$$

onde usamos que $\omega\left(X_{H}(\rho), d \mathscr{P}(y) u\right)=\omega\left(X_{H}(\rho), d \mathscr{P}(y) v\right)=0$ pois $\mathscr{P}(y) u, \mathscr{P}(y) v \in T_{y} \Sigma \subset T_{y} S$.

Escolha uma seção $\Sigma=\Sigma^{\prime} \cap H^{-1}(1)$ transversal a $c$ em x. Então, existem vizinhanças menores $\Sigma_{0}$ e $\Sigma_{1}$ de $\mathrm{x}$ em $\Sigma$, pelo Teorema da função Inversa, e uma função $\tau: \Sigma_{0} \rightarrow \mathbb{R}$ tal que

$$
\mathscr{P}(y)=\psi_{\tau(y)}(y): \Sigma_{0} \rightarrow \Sigma_{1}
$$

é um difeormorfismo que é simplético com respeito à $i^{*} \omega$ (Teorema 6.8).

Em geral, $P=d \mathscr{P}$ difere de $d \psi_{T}(x)$ uma vez que $T_{x} \Sigma$ não é necessariamente invariante sob $d \psi_{T}(x)$. Mas, se $H$ é homogêneo de grau 1 então podemos encontrar um $\Sigma$ tal que $T_{x} \Sigma$ é invariante sob $d \psi_{T}(x)$, como veremos a seguir.

Lema 6.9. Seja $(M, \omega)$ uma variedade simplética e $H: M \rightarrow \mathbb{R}$ um hamiltoniano com campo $X_{H}$ e fluxo $\psi_{t}$. Se H é homogêneo de grau 1 então

$$
\psi_{t}(\lambda y)=\lambda \psi_{\frac{t}{\lambda}}(y)
$$


Demonstração. Como $H$ é homogêneo de grau 1 então para todo $\lambda \in \mathbb{R}$ e $y \in M$ temos

$$
H(\lambda y)=\lambda H(y)
$$

Diferenciando a equação (6.5) obtemos

$$
d H(\lambda y)(\lambda v)=\lambda d H(y) \cdot v
$$

para todo $v \in T_{y} M$ e pela definição do campo de vetores $X_{H}$ segue-se que

$$
\omega\left(X_{H}(\lambda y), \lambda v\right)=\lambda \omega\left(X_{H}(y), v\right)=\omega\left(X_{H}(y), \lambda v\right) \Longrightarrow \omega\left(X_{H}(\lambda y)-X_{H}(y), \lambda v\right)=0
$$

Então, como $\omega$ é não-degenerada segue que

$$
X_{H}(\lambda y)=X_{H}(y)
$$

para todo $y \in M$ e $\lambda \in \mathbb{R}$. Além disso,

$$
\psi_{0}(\lambda y)=\lambda y=\lambda \psi_{\frac{0}{\lambda}}(y)
$$

e pela equação (6.6) temos

$$
\left.\frac{d}{d t} \psi_{t}(\lambda y)\right|_{t=0}=X_{H}(\lambda y)=X_{H}(y)=\left.\frac{d}{d t}\left[\lambda \psi_{\frac{t}{\lambda}}(y)\right]\right|_{t=0} .
$$

Assim, como $\psi_{t}(\lambda y)$ e $\lambda \psi_{\frac{t}{\lambda}}(y)$ tem as mesmas condições iniciais segue pela unicidade das soluções das equações diferenciais do campo $X_{H}$ que

$$
\psi_{t}(\lambda y)=\lambda \psi_{\frac{t}{\lambda}}(y)
$$

Lema 6.10. Seja $(M, \omega)$ uma variedade simplética e $H$ um hamiltoniano homogêneo de grau 1 com campo $X_{H}$ e fluxo $\psi_{t}$. Então, existe uma seção transversal $\Sigma$ na superfície de energia $H^{-1}(1)$ tal que $T_{x} \Sigma$ é invariante sob $d \psi_{T}(x)$.

Demonstração. Diferenciando (6.4) com respeito a $\lambda$ e fazendo $y=x, t=T$ e $\lambda=1$ temos

$$
d \psi_{T}(x) \cdot \widetilde{x}=\psi_{T}(x)-T \cdot X_{H}\left(\psi_{T}(x)\right)=\widetilde{x}-T \cdot \dot{c}(0)
$$

onde $\widetilde{x}$ é igual a $x$ olhado como um vetor tangente em $x$. Mas também como $\psi_{t} \circ \psi_{s} x=\psi_{s} \circ \psi_{t} x$ temos

$$
\left.\frac{d}{d s}\left[\psi_{t} \circ \psi_{s} x\right]\right|_{s=0}=\left.\frac{d}{d s}\left[\psi_{s} \circ \psi_{t} x\right]\right|_{s=0} \Longrightarrow d \psi_{t}(x) \cdot X_{H}(x)=X_{H}\left(\psi_{t}(x)\right)
$$

e fazendo $t=T$ obtemos

$$
d \psi_{T}(x) \cdot \dot{c}(0)=X_{H}\left(\psi_{T}(x)\right)=X_{H}(x)=\dot{c}(0) .
$$

Assim, pelas equações (6.7) e (6.8) segue que $\widetilde{x}$ e $\dot{c}(0)$ formam um subespaço invariante sob $d \psi_{T}(x)$. Além disso, esse subespaço é não-degenerado com respeito a $\omega$ pois diferenciando $H(\lambda x)=\lambda H(x)$ com respeito a $\lambda$ e fazendo $\lambda=1$ temos

$$
d H(x) \cdot \widetilde{x}=H(x)=1
$$

Logo,

$$
\omega(\dot{c}(0), \widetilde{x})=d H(x) \cdot \widetilde{x}=1 .
$$

Sejam A o subespaço gerado por $\widetilde{x}$ e $\dot{c}(0)$ e E o complemento ortogonal de A com respeito a $\omega$, isto é,

$$
E:=A^{\omega}=\left\{v \in T_{x} M ; \omega(v, \widetilde{x})=\omega(v, \dot{c}(0))=0\right\}
$$


Então:

Afirmação 6.11. $d \psi_{T}(x)$ é invariante sob $E$.

Demonstração. Como $d \psi_{T}(x)$ preserva a forma simplética (Teorema 6.8) e pelas equações (6.7) e (6.8) temos $\tilde{x}=d \psi_{T}(x) \cdot \tilde{x}+T \cdot \dot{c}(0)$ e $d \psi_{T}(x) \cdot \dot{c}(0)=\dot{c}(0)$ então para todo $v \in E$ segue que

$$
\begin{aligned}
\omega\left(d \psi_{T}(x) \cdot v, \widetilde{x}\right) & =\omega\left(d \psi_{T}(x) \cdot v, d \psi_{T}(x) \cdot \widetilde{x}+T \cdot \dot{c}(0)\right) \\
& =\omega\left(d \psi_{T}(x) \cdot v, d \psi_{T}(x) \cdot \widetilde{x}+T d \psi_{T}(x) \cdot \dot{c}(0)\right) \\
& =\omega\left(d \psi_{T}(x) \cdot v, d \psi_{T}(x)(\widetilde{x}+T \cdot \dot{c}(0))\right) \\
& =\omega(v, \widetilde{x}+T \cdot \dot{c}(0)) \\
& =\omega(v, \widetilde{x})+T \cdot \omega(v, \dot{c}(0)) \\
& =0
\end{aligned}
$$

e

$$
\omega\left(d \psi_{T}(x) \cdot v, \dot{c}(0)\right)=\omega\left(d \psi_{T}(x) \cdot v, d \psi_{T}(x) \cdot \dot{c}(0)\right)=\omega(v, \dot{c}(0))=0
$$

Logo, $d \psi_{T}(x) \cdot v \in E$ para todo $v \in E$ e, portanto, $d \psi_{T}(x) \cdot v$ é invariante sob E.

Afirmação 6.12. O subespaço E é não-degenerado.

Demonstração. Suponha que exista $v \in E$ não nulo tal que $\omega(v, u)=0$ para todo $u \in E$ então $v \in E^{\omega}=A$. Assim, $v$ é um elemento não nulo do subespaço gerado por $\widetilde{x}$ e $\dot{c}(0)$ tal que

$$
\omega(v, \widetilde{x})=\omega(v, \dot{c}(0))=0 .
$$

Mas isso é uma contradição ao fato de A ser um subespaço não degenerado. Logo, se $\omega(v, u)=0$ para todo $u \in E$ então $v=0$, isto é, E é um subespaço não-degenerado.

Assim, como $E$ é não-degenerada e $\omega\left(v, X_{H}(x)\right)=\omega(v, \dot{c}(0))=0$ para todo $v \in E$ temos

$$
E \cap \operatorname{span}\left\{X_{H}(x)\right\}=0 .
$$

Além disso, como:

(1) $T_{x} M=A+A^{\omega}=A+E$,

(2) $T_{x} H^{-1}(1)=\left\{v \in T_{x} M ; \omega\left(X_{H}(x), v\right)=0\right\}$ por (6.2) $\mathrm{e}$

(3) $E \subset T_{x} H^{-1}(1), X_{H}(x) \in T_{x} H^{-1}(1)$ e $\widetilde{x} \notin T_{x} H^{-1}(1)$ pois $\omega\left(\widetilde{x}, X_{H}(x)\right)=1$ pela equação (6.9)

concluímos que

$$
T_{x} H^{-1}(1)=E \oplus \operatorname{span}\left\{X_{H}(x)\right\} .
$$

Portanto, como E é invariante sob $d \psi_{T}(x)$ e vale (6.10), escolhendo $\Sigma$ tal que $T_{x} \Sigma=\mathrm{E}$, então

$$
P=\left.d \psi_{T}(x)\right|_{E} .
$$

O fluxo linearizado $d \psi_{T}(x)$ pode ser entendido da seguinte forma: Escolhendo uma variação de curvas solução $c_{s}(t):=c(s, t), c_{0}(t)=c(t)$, e definindo o vetor variação

$$
Y(t)=\left.\frac{\partial c_{s}}{\partial s}\right|_{s=0}
$$


tem-se que $Y(t)$ é uma solução do fluxo linearizado

$$
d \psi_{t}(Y(0))=Y(t)
$$

e, assim, $P(Y(0))=Y(T)$. Para computar $Y(t)$ escolhemos um sistema de coordenadas $p_{1}, \ldots, p_{n}$ em M tal que $p_{2}=\cdots=p_{n}=0$ ao longo de $c(t), t=p_{1}$ e $p_{1}$ periódico, $p_{1}(t+T)=p_{1}(t)$ (é sempre possível encontrar tal sistema de coordenadas no caso hamiltoniano).

Seja $\left(p_{i}, q_{i}\right)$ o sistema de coordenadas induzido em $T^{*} M$. Uma vez que $c_{s}$ é uma solução das equações de Hamilton $\dot{p}_{i}=H_{q_{i}}, \dot{q}_{i}=-H_{p_{i}}$, então se

$$
Y(t)=\left.\frac{\partial c_{s}}{\partial s}\right|_{s=0}=\left(\frac{\partial p_{i}}{\partial s}(0, t), \frac{\partial q_{i}}{\partial s}(0, t)\right)=\left(\xi_{i}(t), \eta_{i}(t)\right)
$$

temos

$$
\begin{aligned}
\left(\dot{\xi}_{i}(t), \dot{\eta}_{i}(t)\right) & =\left.\frac{\partial^{2} c_{s}}{\partial t \partial s}(t)\right|_{s=0} \\
& =\frac{\partial}{\partial s}\left[\left(\dot{p}_{i}(s, t), \dot{q}_{i}(s, t)\right)\right]_{s=0} \\
& =\frac{\partial}{\partial s}\left[\left(H_{q_{i}}\left(p_{i}(s, t), q_{i}(s, t)\right),-H_{p_{i}}\left(p_{i}(s, t), q_{i}(s, t)\right)\right)\right]_{s=0} \\
& =\left(H_{q_{i} p_{j}} \cdot \frac{\partial p_{j}}{\partial s}(0, t)+H_{q_{i} q_{j}} \cdot \frac{\partial q_{j}}{\partial s}(0, t),-H_{p_{i} p_{j}} \cdot \frac{\partial p_{j}}{\partial s}(0, t)-H_{p_{i} q_{j}} \cdot \frac{\partial q_{j}}{\partial s}(0, t)\right) \\
& =\left(H_{q_{i} p_{j}} \cdot \xi_{j}(t)+H_{q_{i} q_{j}} \cdot \eta_{j}(t),-H_{p_{i} p_{j}} \cdot \xi_{j}(t)-H_{p_{i} q_{j}} \cdot \eta_{j}(t)\right)
\end{aligned}
$$

Logo,

$$
\left\{\begin{array}{l}
\dot{\xi}_{i}=H_{q_{i} p_{j}} \cdot \xi_{j}(t)+H_{q_{i} q_{j}} \cdot \eta_{j}(t) \\
\dot{\eta}_{i}=-H_{p_{i} p_{j}} \cdot \xi_{j}(t)-H_{p_{i} q_{j}} \cdot \eta_{j}(t)
\end{array}\right.
$$

onde as derivadas parciais de $\mathrm{H}$ são calculadas ao longo de $c(t)$.

Teorema 6.13. A aplicação linearizada de Poincaré das geodésicas fechadas em $S^{2}$ são rotações com ângulos $2 \pi /(1+\alpha)$ e $2 \pi /(1-\alpha)$ respectivamente. Em particular, para $\alpha$ irracional todas as geodésicas fechadas são elípticas com expoente irracional.

Demonstração. Seja $H=\frac{1}{2} H_{\alpha}^{2}$ onde $\alpha<1$ é irracional. Pelo teorema 4.28 segue que o fluxo associado à $X_{H}$ é o fluxo co-geodésico de $F_{\alpha}$. Tome a parametrização de $S^{2}$

$$
\begin{aligned}
x & =\cos p_{1} \cos p_{2} \\
y & =\sin p_{1} \cos p_{2} \\
z & =\sin p_{2}
\end{aligned}
$$

Seja $c_{1}(t)$ o "equador"de $S^{2}$, isto é, $c_{1}(t)=\left(p_{1}(t), 0\right)$. Lembre que na métrica $F_{\alpha}$, com $\alpha$ irracional, as únicas geodésicas periódicas que existem são aquelas obtidas percorrendo o "equador"nas direções horária e anti-horária. Sobre essa curva temos $\frac{\partial}{\partial p_{1}}=V$ e $\frac{\partial}{\partial p_{2}}$ ortogonal a $V$, além disso, $V$ e $\frac{\partial}{\partial p_{2}}$ tem comprimento unitário com respeito a métrica $g$. Seja $H_{1}$ como definido em (4.5). Com a parametrização (6.14) temos

$$
H_{1}\left(p_{i}, q_{i}\right)=q_{1} d p_{1}(V)+q_{2} d p_{2}(V)=q_{1} d p_{1}\left(\frac{\partial}{\partial p_{1}}\right)+q_{2} d p_{2}\left(\frac{\partial}{\partial p_{1}}\right)=q_{1}
$$

e a matriz $g_{i j}$ associada a métrica g é:

$$
g_{i j}=\left(\begin{array}{cc}
\cos ^{2} p_{2} & 0 \\
0 & 1
\end{array}\right) \Longrightarrow g^{i j}=\left(\begin{array}{cc}
\sec ^{2} p_{2} & 0 \\
0 & 1
\end{array}\right) .
$$


Logo,

$$
\begin{aligned}
H\left(p_{i}, q_{i}\right) & =\frac{1}{2} H_{\alpha}^{2}\left(p_{i}, q_{i}\right) \\
& =\frac{1}{2}\left[\sqrt{g^{i j} q_{i} q_{j}}+\alpha q_{i}\right]^{2} \\
& =\frac{1}{2}\left[\sqrt{\left(\sec ^{2} p_{2}\right) q_{1}^{2}+q_{2}^{2}}+\alpha q_{1}\right]^{2}
\end{aligned}
$$

As órbitas periódicas $c(t)$ de $X_{H}$ com curva base $c_{1}(t)$ e $H_{\alpha}(c(t))=1$ são tais que:

$$
c(t)=\left(p_{1}(t), p_{2}(t), q_{1}(t), q_{2}(t)\right)
$$

onde $p_{2}(t) \equiv 0$. Queremos descobrir quem são $p_{1}(t), q_{1}(t)$ e $q_{2}(t)$. Como $c(t)$ é solução das equações de Hamilton associadas a $\mathrm{H}$ temos:

$$
\begin{aligned}
& \dot{p}_{1}(t)=\frac{\partial H}{\partial q_{1}}=H_{\alpha}(c(t)) \cdot\left[\frac{\left(\sec ^{2} p_{2}\right) q_{1}}{\sqrt{\left(\sec ^{2} p_{2}\right) q_{1}^{2}+q_{2}^{2}}}+\alpha\right]=\frac{q_{1}}{\sqrt{q_{1}^{2}+q_{2}^{2}}}+\alpha \\
& \dot{p}_{2}(t)=\frac{\partial H}{\partial q_{2}}=H_{\alpha}(c(t)) \cdot\left[\frac{q_{2}}{\sqrt{\left(\sec ^{2} p_{2}\right) q_{1}^{2}+q_{2}^{2}}}\right]=\frac{q_{2}}{\sqrt{q_{1}^{2}+q_{2}^{2}}} \\
& \dot{q}_{1}(t)=-\frac{\partial H}{\partial p_{1}}=0 \\
& \dot{q}_{2}(t)=-\frac{\partial H}{\partial p_{2}}=H_{\alpha}(c(t)) \cdot\left[\frac{\left(\sec ^{2} p_{2}\right)\left(\tan p_{2}\right) q_{1}^{2}}{\sqrt{\left(\sec ^{2} p_{2}\right) q_{1}^{2}+q_{2}^{2}}}\right]=0
\end{aligned}
$$

Como $p_{2}(t) \equiv 0$ temos $\dot{p}_{2}(t) \equiv 0$. Assim, da equação (6.14d) segue que $q_{2}(t)$ é constante e de (6.14b) temos

$$
0=\frac{q_{2}}{\sqrt{q_{1}^{2}+q_{2}^{2}}} \Longrightarrow q_{2}(t) \equiv 0
$$

A equação (6.14c) nos diz que $q_{1}(t)$ é constante. Substituindo (6.15) na equação (6.14a) obtemos

$$
\dot{p}_{1}(t)=\frac{q_{1}}{\left|q_{1}\right|}+\alpha
$$

Se $c_{1}$ é a geodésica de comprimento $T=2 \pi /(1+\alpha)$ então $q_{1}>0$. Assim, temos

$$
\dot{p}_{1}(t)=1+\alpha \Longrightarrow p_{1}(t)=(1+\alpha) t .
$$

Como $H_{\alpha}(c(t))=1$ e $q_{2}=0$ então

$$
1=\sqrt{\left(\sec ^{2} p_{2}\right) q_{1}^{2}+q_{2}^{2}}+\alpha q_{1}=\left|q_{1}\right|+\alpha q_{1} \Longrightarrow(1+\alpha) q_{1}=1 \Longrightarrow q_{1}=\frac{1}{1+\alpha} .
$$

Logo,

$$
c(t)=\left((1+\alpha) t, 0, \frac{1}{1+\alpha}, 0\right) .
$$


Calculando as derivadas de segunda ordem de $H$ ao longo de $c(t)$ temos

$$
\begin{aligned}
H_{q_{1} q_{1}} & =(1+\alpha)^{2} \\
H_{q_{2} q_{2}} & =1+\alpha \\
H_{q_{1} q_{2}} & =H_{p_{1} p_{1}}=H_{p_{1} p_{2}}=0 \\
H_{p_{2} p_{2}} & =\frac{1}{1+\alpha}
\end{aligned}
$$

Logo, as equações diferenciais para $Y(t)$ são:

$$
\begin{array}{ll}
\dot{\xi}_{1}=(1+\alpha)^{2} \eta_{1} & \dot{\eta}_{1}=0 \\
\dot{\xi}_{2}=(1+\alpha) \eta_{2} & \dot{\eta}_{2}=-\frac{1}{1+\alpha} \cdot \xi_{2}
\end{array}
$$

Como E é o $\omega$-complemento ortogonal gerado por $\dot{c}(0)=(1+\alpha) \frac{\partial}{\partial p_{1}}$ e $\widetilde{x}=\widetilde{c(0)}=\frac{1}{1+\alpha} \frac{\partial}{\partial q_{1}}$ então se

$$
v=v_{1} \frac{\partial}{\partial p_{1}}+v_{2} \frac{\partial}{\partial p_{2}}+v_{3} \frac{\partial}{\partial q_{1}}+v_{4} \frac{\partial}{\partial q_{2}} \in E
$$

temos

$$
\omega\left(v, \frac{\partial}{\partial p_{1}}\right)=\omega\left(v, \frac{\partial}{\partial q_{1}}\right)=0
$$

onde $\omega=d p_{1} \wedge d q_{1}+d p_{2} \wedge d q_{2}$. Assim,

$$
0=\omega\left(v, \frac{\partial}{\partial p_{1}}\right)=d p_{1} \wedge d q_{1}\left(v, \frac{\partial}{\partial p_{1}}\right)+d p_{2} \wedge d q_{2}\left(v, \frac{\partial}{\partial p_{1}}\right)=-v_{3}
$$

e

$$
0=\omega\left(v, \frac{\partial}{\partial q_{1}}\right)=d p_{1} \wedge d q_{1}\left(v, \frac{\partial}{\partial q_{1}}\right)+d p_{2} \wedge d q_{2}\left(v, \frac{\partial}{\partial q_{1}}\right)=v_{1}
$$

Logo, $v=v_{2} \frac{\partial}{\partial p_{2}}+v_{4} \frac{\partial}{\partial q_{2}}$ e isto implica que E é gerado por $\frac{\partial}{\partial p_{2}} \mathrm{e} \frac{\partial}{\partial q_{2}}$. Observe que

$$
\begin{aligned}
\omega\left(\sqrt{1+\alpha} \cdot \frac{\partial}{\partial p_{2}}, \frac{1}{\sqrt{1+\alpha}} \cdot \frac{\partial}{\partial q_{2}}\right) & =\left(d p_{1} \wedge d q_{1}+d p_{2} \wedge d q_{2}\right)\left(\sqrt{1+\alpha} \cdot \frac{\partial}{\partial p_{2}}, \frac{1}{\sqrt{1+\alpha}} \cdot \frac{\partial}{\partial q_{2}}\right) \\
& =\frac{\sqrt{1+\alpha}}{\sqrt{1+\alpha}} \\
& =1
\end{aligned}
$$

Logo, $\mathscr{B}=\left\{\sqrt{1+\alpha} \cdot \frac{\partial}{\partial p_{2}}, \frac{1}{\sqrt{1+\alpha}} \cdot \frac{\partial}{\partial q_{2}}\right\}$ é uma base simplética para o subespaço E. Além disso, resolvendo o sistema

$$
\left\{\begin{array}{l}
\dot{\xi}_{2}=(1+\alpha) \cdot \eta_{2} \\
\dot{\eta}_{2}=-\frac{1}{1+\alpha} \cdot \xi_{2}
\end{array}\right.
$$

obtemos

$$
\begin{aligned}
& \xi_{2}(t)=a \sqrt{1+\alpha} \cdot \cos t+b \sqrt{1+\alpha} \cdot \sin t \\
& \eta_{2}(t)=b \frac{1}{\sqrt{1+\alpha}} \cdot \cos t-a \frac{1}{\sqrt{1+\alpha}} \cdot \sin t
\end{aligned}
$$


onde $a, b \in \mathbb{R}$. Escrevendo os vetores $Y(0)$ e $Y(T)$ na base simplética $\mathscr{B}$ obtemos

$$
\begin{aligned}
& Y(0)=\left(\frac{1}{\sqrt{1+\alpha}} \cdot \xi_{2}(0), \sqrt{1+\alpha} \cdot \eta_{2}(0)\right)=(a \cos 0+b \sin 0, b \cos 0-a \sin 0)=(a, b) \\
& Y(T)=\left(\frac{1}{\sqrt{1+\alpha}} \cdot \xi_{2}(T), \sqrt{1+\alpha} \cdot \eta_{2}(T)\right)=(a \cos T+b \sin T, b \cos T-a \sin T)
\end{aligned}
$$

Como $P$ é tal que $P(Y(0))=Y(T)$ temos

$$
P(a, b)=(a \cos T+b \sin T, b \cos T-a \sin T)
$$

isto é,

$$
P\left[\begin{array}{l}
a \\
b
\end{array}\right]=\left[\begin{array}{cc}
\cos T & \sin T \\
-\sin T & \cos T
\end{array}\right]\left[\begin{array}{l}
a \\
b
\end{array}\right]
$$

Logo,

$$
P=\left[\begin{array}{cc}
\cos T & \sin T \\
-\sin T & \cos T
\end{array}\right]
$$

e, portanto, P é uma rotação com ângulo $T=2 \pi /(1+\alpha)$. Para a geodésica de comprimento $T=2 \pi /(1-\alpha)$, concluímos de forma análoga que $P$ é uma rotação com ângulo $T=2 \pi /(1-\alpha)$. Por fim, precisamos calcular os autovalores de $P$. Se $\lambda$ é tal que $\operatorname{det}(P-\lambda I d)=0$ temos

$$
\operatorname{det}\left[\begin{array}{cc}
\cos T-\lambda & \sin T \\
-\sin T & \cos T-\lambda
\end{array}\right]=0 \Longrightarrow \lambda^{2}-2 \cos T \cdot \lambda+1=0
$$

Resolvendo a equação acima em relação a $\lambda$ obtemos

$$
\lambda=\frac{2 \cos T \pm \sqrt{4 \cos ^{2} T-4}}{2}=\cos T \pm i \sin T=e^{ \pm i T} .
$$

Portanto, as geodésicas de comprimentos $T=2 \pi /(1+\alpha)$ e $T=2 \pi /(1-\alpha)$ são elípticas com expoente irracional, se $\alpha$ for irracional.

\section{3 Índice de Morse dos Exemplos de Katok}

Agora vamos mostrar que se pode dar uma descrição da Teoria de Morse do funcional energia

$$
E(c)=\frac{1}{2} \int F^{2}(c(t), \dot{c}(t)) d t
$$

no espaço de curvas suaves por partes $\Lambda=C^{\infty}\left(S^{1}, M\right)$ (i.e $c:[0,1] \rightarrow M$ tal que $c(0)=c(1)$ ). Faremos os cálculos, novamente, só para $M=S^{2}$, as outras variedades são similares. Denote por $c_{1}$ a geodésica fechada curta de comprimento $2 \pi /(1+\alpha)$ e por $c_{2}$ a geodésica fechada longa de comprimento $2 \pi /(1-\alpha)$. Mas antes de analisarmos os índices de Morse dos exemplos de Katok em $S^{2}$ veremos alguns resultados importantes sobre a Teoria de Morse, onde parte dos resultados foram retirados ou adaptados de [Mil63] e [BTZ82].

Notação 6.14. Seja $V_{\Lambda}(c)$ o espaço de campos de vetores suaves por partes ao longo de $c$ tal que

$$
g_{\dot{c}(t)}(X(t), \dot{c}(t))=0
$$

para todo $t$ e tal que $X(1)=X(0)$.

Definição 6.15. O índice de um funcionamento bilinear $H$ em um espaço vetorial $V$, é definido como a dimensão do subespaço maximal de $V$ em que $H$ é negativa-definida. A nulidade é a dimensão do núcleo de $H$, isto é, o subespaço de todos os elementos de $v \in V$ tais que $H(v, w)=0$ para todo $w \in V$. 
Definição 6.16. Definimos $I_{c}: V_{\Lambda}(c) \times V_{\Lambda}(c) \rightarrow \mathbb{R}$ por

$$
\begin{aligned}
I_{c}(X, Y)= & -\int_{0}^{1} g_{\dot{c}}\left(D_{\dot{c}} D_{\dot{c}} X+R_{\dot{c}}(X), Y\right) d t-\sum_{i=1}^{k-1} g_{\dot{c}\left(t_{i}\right)}\left(D_{\dot{c}} X\left(t_{i}^{+}\right)-D_{\dot{c}} X\left(t_{i}^{-}\right), Y(t)\right) \\
& +g_{\dot{c}(1)}\left(D_{\dot{c}} X\left(1^{-}\right), Y(1)\right)-g_{\dot{c}(0)}\left(D_{\dot{c}}\left(0^{+}\right), Y(0)\right)
\end{aligned}
$$

onde $0=t_{0}<t_{1}<\ldots<t_{k}=1$ são pontos de descontinuidade de $D_{\dot{c}} X$.

Definição 6.17. O índice de $c$ como uma geodésica fechada é definido como o índice de $I_{c}$. Denotaremos o índice de $c$ por ind $(c)$. A nulidade de $c$ é definida como dim $\operatorname{ker}\left(\left.I_{c}\right|_{V_{\Lambda(c)}}\right)$ e o denotaremos por nul $(c)$.

Observação 6.18. Pela mesma demostração do Lema 3.8.2 em [SH13] obtemos a igualdade $I_{c}=\left.d_{c}^{2} E\right|_{V_{\Lambda(c)}}$.

Observação 6.19. Note que a nulidade de $c$ como um ponto crítico de $E$ é nul $(c)+1$ pois $\dot{c}$ pertence ao núcleo da hessiana de $E$.

Definição 6.20. O índice da geodésica não-periódica $c:[0,1] \rightarrow M$ denotada por ind ${ }_{\Omega}(c)$, é definida como o índice da forma $I_{c}$ definida no espaço $V_{\Omega}(c)$ dos campos de vetores suaves por partes $X$ ao longo de $c$ tal que

$$
g_{\dot{c}(t)}(X(t), \dot{c}(t))=0
$$

para todo $t$ e tal que $X(1)=X(0)=0$.

Definição 6.21. Definimos a nulidade da geodésica não periódica $c:[0,1] \rightarrow M$ como a dimensão do núcleo de $\left.I_{c}\right|_{V_{\Omega}(c)}$ e a denotamos por nul $\Omega$, isto é, $\operatorname{nul}_{\Omega}:=\operatorname{dim} \operatorname{ker}\left(\left.I_{c}\right|_{V_{\Omega}(c)}\right)$.

Observação 6.22. Seja $\mu(t)$ o número de campos de Jacobi linearmente independentes $Y$ ao longo $c$ com $Y(0)=Y(t)=0$. Então, pelo Teorema 14.1 e pelo Teorema 15.1 em [Mil63] obtemos

$$
\operatorname{nul}_{\Omega}(c)=\mu(1) \mathrm{e} \operatorname{ind}_{\Omega}(c)=\sum_{0<t<1} \mu(t)
$$

Teorema 6.23. A nulidade de $c \in \Lambda$ é igual ao número máximo de campos de Jacobi linearmente independentes $Y$ ortogonal a ć com respeito a $g_{\dot{c}}$, isto é, campos de Jacobi com $Y(0)=Y(1), D_{\dot{c}} Y(0)=D_{\dot{c}} Y(1) e$ $g_{\dot{c}}(Y(t), \dot{c})=0$ para todo $t$.

Demonstração. Seja $J$ um campo de Jacobi tal que $J(0)=J(1), D_{\dot{c}} J(0)=D_{\dot{c}} J(1)$ e $g_{\dot{c}}(J(t), \dot{c})=0$. Então, $J \in V_{\Lambda(c)} \mathrm{e}$

$$
I_{c}(X, Y)=-\int_{0}^{1} g_{\dot{c}}(0, Y) d t-\sum_{i=1}^{k-1} g_{\dot{c}\left(t_{i}\right)}(0, Y(t))+g_{\dot{c}(1)}(0, Y(1)-Y(0))=0 .
$$

Logo, $J \in \operatorname{ker} I_{c}$. Por outro lado, seja $J$ um campo de vetores no núcleo de $I_{c}$. Escolha uma partição $0=t_{0}<$ $t_{1}<\ldots<t_{k}=1$ de $[0,1]$ tal que $J \mid\left[t_{i-1}, t_{i}\right]$ é suave para cada $i=1, \ldots, k-1$. Seja $f:[0,1] \rightarrow[0,1]$ uma função suave que se anula em $t_{0}, t_{1}, \ldots, t_{k}$ e é positiva nos outros pontos. Seja

$$
J_{2}(t)=f(t)\left(D_{\dot{c}} D_{\dot{c}} J+R_{\dot{c}}(J)\right) .
$$

Então, como

$$
0=I_{c}\left(J, J_{2}\right)=-\int_{0}^{1} f(t) g_{\dot{c}}\left(D_{\dot{c}} D_{\dot{c}} J+R_{\dot{c}}(J), D_{\dot{c}} D_{\dot{c}} J+R_{\dot{c}}(J)\right) d t+0
$$

obtemos que $J \mid\left[t_{i-1}, t_{i}\right]$ é um campo de Jacobi para cada $i=1, \ldots, k-1$. Agora, seja $J_{2}^{\prime} \in V_{\Lambda(c)}$ um campo tal que $J_{2}^{\prime}\left(t_{i}\right)=D_{\dot{c}} J\left(t_{i}^{+}\right)-D_{\dot{c}} J\left(t_{i}^{-}\right)$para $i=1, \ldots, k$. Então,

$$
0=I_{c}\left(J, J_{2}^{\prime}\right)=0-\sum_{i=1}^{k} g_{\dot{c}\left(t_{i}\right)}\left(D_{\dot{c}} J\left(t_{i}^{+}\right)-D_{\dot{c}} J\left(t_{i}^{-}\right), D_{\dot{c}} J\left(t_{i}^{+}\right)-D_{\dot{c}} J\left(t_{i}^{-}\right)\right)
$$


e isto implica que $D_{\dot{c}} J$ é contínuo. Portanto, $J$ é um campo de Jacobi suave.

Observação 6.24. O espaço $V_{\Lambda}(c)$ é a soma direta de $V_{\Lambda}^{1}(c)$ e $V_{\Lambda}^{2}(c)$, onde

$$
\begin{aligned}
V_{\Lambda}^{1}(c) & =\left\{X \in V_{\Lambda}(c) ; X \mid\left[t_{i}, t_{i+1}\right] \text { é um campo de Jacobi }\right\} \\
V_{\Lambda}^{2}(c) & =\left\{X \in V_{\Lambda}(c) ; X\left(t_{i}\right)=0,0 \leq i \leq k\right\}
\end{aligned}
$$

Além disso, $V_{\Lambda}^{1}(c)$ e $V_{\Lambda}^{2}(c)$ são ortogonais com respeito a $H$ e $H$ é positivo-definido em $V_{\Lambda}^{2}(c)$ (ver Lema $15.3 \mathrm{em}$ [Mil63]). Observe que ind $(c)$ é o índice de $I_{c}$ em $V_{\Lambda}^{1}(c)$ e portanto é finito.

Teorema 6.25. Seja $H$ uma forma simétrica definida em um espaço vetorial de dimensão finita $V$ (ou forma Hermitiana em um espaço vetorial complexo). Para qualquer subespaço $W \subset V$ temos

(a) ind $H=\left.\operatorname{ind} H\right|_{W}+\left.\operatorname{ind} H\right|_{W^{\perp}}+\operatorname{dim}\left(W \cap W^{\perp}\right)-\operatorname{dim}(W \cap \operatorname{ker} H)$

(b) $\operatorname{dim} \operatorname{ker} H=\left.\operatorname{dim} \operatorname{ker} H\right|_{W^{\perp}}-\operatorname{dim}\left(W \cap W^{\perp}\right)+\operatorname{dim}(W \cap \operatorname{ker} H)$

onde $W^{\perp}=\{X \in V ; H(X, Y)=0, \forall Y \in W\}$.

Para uma prova do resultado acima veja [BTZ82], página 218. Usando a Observação 6.22 e o Teorema 6.25 obtemos um importante teorema que caracteriza o índice de uma geodésicas fechadas.

Teorema 6.26. $O$ índice e a nulidade das geodésicas fechadas satisfazem a equação

$$
\operatorname{ind}(c)=\operatorname{ind}_{\Omega}(c)+\left.(\text { ind }+\operatorname{dimker}) I_{c}\right|_{W^{\perp}}-\operatorname{nul}(c)
$$

onde $W^{\perp}$ é o subespaço ortogonal, com respeito a $I_{c}$, do subespaço $V_{\Lambda}^{1}(c) \cap V_{\Omega}(c)$.

Demonstração. Escolha pontos $0=t_{0}<t_{1}<\ldots<t_{k}=1$ tais que não existam pontos conjugados no interior de $c \mid\left[t_{i}, t_{i+1}\right]$. Aplicaremos o item (a) do Teorema 6.25 para $V=V_{\Lambda}^{1}(c), W=V_{\Lambda}^{1}(c) \cap V_{\Omega}(c)$ e $H=I_{c}$. Como ind $\left.I_{c}\right|_{W}=\operatorname{ind}_{\Omega}(c)$ e da equação (6.20) segue que $W^{\perp}$ consiste de campos de Jacobi suaves $Y$ ao longo de $c$ com $Y(0)=Y(1)$, mas possivelmente $D_{\dot{c}} Y(0) \neq D_{\dot{c}} Y(1)$ segue que $\operatorname{dim}\left(W \cap W^{\perp}\right)=\mu(1)$ e $W \cap \operatorname{ker} I_{c}$ são os campos de Jacobi periódicos que se anulam em 0 . Para $X, Y \in W^{\perp}$ temos

$$
I_{c}(X, Y)=g_{\dot{c}}\left(D_{\dot{c}} Y\left(1^{-}\right)-D_{\dot{c}} Y\left(0^{+}\right), Y(0)\right)
$$

Então, pelo Teorema 6.25 temos

(i) $\operatorname{ind}(c)=\operatorname{ind}_{\Omega}(c)+\left.\operatorname{ind} I_{c}\right|_{W^{\perp}}+\mu(1)-\operatorname{dim}\left(W \cap \operatorname{ker} I_{c}\right)$,

(ii) $\operatorname{dim} \operatorname{ker} I_{c}=\left.\operatorname{dim} \operatorname{ker} I_{c}\right|_{W^{\perp}}-\mu(1)+\operatorname{dim}\left(W \cap \operatorname{ker} I_{c}\right)$.

Logo,

$$
\operatorname{ind}(c)=\operatorname{ind}_{\Omega}(c)+\left.(\text { ind }+\operatorname{dimker}) I_{c}\right|_{W^{\perp}}-\operatorname{nul}(c) .
$$

Definição 6.27. O termo ind $(c)-\operatorname{ind}_{\Omega}(c)$ é chamado a concavidade de $c$ e o denotaremos por conc $(c)$.

Definição 6.28. A iterada $c^{k}$ de uma geodésica $c$ é definida por

$$
c^{k}(t)=c(k t)
$$

Se $c$ é uma geodésica fechada então todas as suas iteradas $c^{k}$ também são geodésicas fechadas e representam círculos críticos diferentes em $\Lambda$.

Teorema 6.29. As geodésicas $c_{1}, c_{2}$ e todas as suas iteradas foram uma subvariedade crítica não-degenerada de $\Lambda$. 
Demonstração. Como vimos no Teorema 6.23, a nulidade de uma geodésica fechada, considerada como um círculo crítico em $\Lambda$, é a dimensão do espaço de campos de Jacobi periódicos (módulo $\dot{c}$ ). Se $\alpha$ é irracional, apenas $c_{1}$ e $c_{2}$ são geodésicas fechadas na métrica $F_{\alpha}$. Logo, os campos de Jacobi periódicos de $F_{\alpha}$ são todos tangentes as geodésicas $c_{1}$ e $c_{2}$, ou seja, $c_{1}$ e $c_{2}$ tem nulidade zero. Pelo mesmo argumento, se $\alpha$ é irracional, todas as iteradas de $c_{1}$ e $c_{2}$ tem nulidade zero.

Se $c_{s}$ é uma família a 1-parâmetro de geodésicas em M, então

$$
Y(t)=\left.\frac{d}{d t}\right|_{s=0} c_{s}(t) \in T_{c(t)} M
$$

é um campo de Jacobi ao longo de $c$ (ver Lema 14.4 em [Mil63]). Segue da computação do fluxo linearizado $D \phi_{t}$ que os campos de Jacobi no nosso exemplo são os campos de vetores com componentes $\xi_{i}$ (ver equação (6.11)) com respeito ao sistema de coordenadas $p_{i}$. Assim, se $F_{\alpha}(c(t), \dot{c}(t))=1$ os campos de Jacobi que não são tangentes a geodésica são da forma:

$$
Y(t)=f(t) \frac{\partial}{\partial p_{2}} .
$$

Como $p_{1}, p_{2}$ são coordenadas de $S^{2}$ tais que $c(t)=\left(p_{1}(t), 0\right)$ (onde $c=c_{1}$ ou $c=c_{2}$ ) e o campo de Jacobi $Y(t)$ satisfaz a equação

$$
D_{\dot{c}} D_{\dot{c}} Y(t)+R_{\dot{c}}(Y)=0
$$

temos

$$
f^{\prime \prime}(t) \frac{\partial}{\partial p_{2}}+f(t) \frac{\partial}{\partial p_{2}}=0 \Longrightarrow f^{\prime \prime}(t)+f(t)=0 .
$$

Logo, o campo $Y$ é da forma:

$$
Y(t)=(A \cos t+B \sin t) \frac{\partial}{\partial p_{2}} \quad A, B \in \mathbb{R}
$$

Pelo Teorema 6.26 obtemos ind $(c)=\operatorname{ind}_{\Omega}(c)+\operatorname{conc}(c)$, onde $\operatorname{conc}(c)=\left.($ ind $+\operatorname{dim} \operatorname{ker}) I_{c}\right|_{W^{\perp}}-\operatorname{nul}(c)$. Para superfícies a concavidade tem a seguinte interpretação geométrica: Se os pontos extremos $t=0$ e $t=T$ não são conjugados, existe um campo de Jacobi $Y$ com

$$
Y(t)=f(t) \frac{\partial}{\partial p_{2}} \quad \text { e } \quad f(0)=f(T)=1
$$

se escolhermos um sistema de coordenadas tal que $c(t)=\left(p_{1}(t), 0\right)$.

Observação 6.30. Veja que:

- Se $f^{\prime}(T)<f^{\prime}(0)$

$$
I_{c}(Y, Y)=g_{\dot{c}}\left(D_{\dot{c}} Y(T)-D_{\dot{c}} Y(0), Y(0)\right)<g_{\dot{c}}\left(D_{\dot{c}} Y(0)-D_{\dot{c}} Y(0), Y(0)\right)=0 .
$$

Logo,

$$
\operatorname{conc}(c)=\left.(\text { ind }+\operatorname{dim} \operatorname{ker}) I_{c}\right|_{W^{\perp}}-\operatorname{nul}(c)=1 .
$$

- Se $f^{\prime}(T) \geq f^{\prime}(0)$

$$
I_{c}(Y, Y)=g_{\dot{c}}\left(D_{\dot{c}} Y(T)-D_{\dot{c}} Y(0), Y(0)\right) \geq g_{\dot{c}}\left(D_{\dot{c}} Y(0)-D_{\dot{c}} Y(0), Y(0)\right)=0 .
$$

Logo,

$$
\operatorname{conc}(c)=\left.(\text { ind }+\operatorname{dimker}) I_{c}\right|_{W^{\perp}}-\operatorname{nul}(c)=0 .
$$

No nosso exemplo, os pontos conjugados ocorrem nos tempos $t=k \cdot \pi, k=1,2, \cdots$. Logo, os pontos 
extremos de $c_{1}^{k}$ e $c_{2}^{k}$ nunca são conjugados. O campo de Jacobi

$$
Y(t)=f(t) \frac{\partial}{\partial p_{2}}
$$

$\operatorname{com} f(0)=f(T)=1$ é da forma

$$
f(t)=\cos t+\frac{1-\cos T}{\sin T} \sin t
$$

Logo,

$$
f^{\prime}(0)=\frac{1-\cos T}{\sin T} \quad \text { e } \quad f^{\prime}(T)=-\frac{1-\cos T}{\sin T} .
$$

Assim,

- Se $0<T$ módulo $2 \pi<\pi$ temos $f^{\prime}(T)<f^{\prime}(0)$ então $\operatorname{conc}(c)=1$.

- Se $\pi<T$ módulo $2 \pi<2 \pi$ temos $f^{\prime}(T)>f^{\prime}(0)$ então $\operatorname{conc}(c)=0$.

Logo, para a geodésica curta $c_{1}$ temos:

$$
\pi<T=\frac{2 \pi}{1+\alpha}<2 \pi \quad \text { e } \quad \operatorname{ind}_{\Omega}\left(c_{1}\right)=1
$$

e isto implica que ind $\left(c_{1}\right)=1$ para todo $\alpha \operatorname{com} 0<\alpha<1$. Mas para $c_{2}$ o ind $\left(c_{2}\right)$ depende de $\alpha$, pois a medida que $\alpha$ se aproxima de $1, \operatorname{ind}_{\Omega}\left(c_{2}\right) \rightarrow \infty$. Se $0<\alpha<1 / 2$ temos

$$
2 \pi<T=\frac{2 \pi}{1-\alpha}<4 \pi \text {. }
$$

Assim,

- Se $2 \pi<\frac{2 \pi}{1-\alpha}<3 \pi$ então $\operatorname{conc}\left(c_{2}\right)=1$ e ind $\operatorname{in}_{\Omega}\left(c_{2}\right)=2$. Logo, $\operatorname{ind}\left(c_{2}\right)=3$.

- Se $3 \pi<\frac{2 \pi}{1-\alpha}<4 \pi$ então $\operatorname{conc}\left(c_{2}\right)=0$ e ind $\operatorname{in}_{\Omega}\left(c_{2}\right)=3$. Logo, ind $\left(c_{2}\right)=3$.

Ou seja, quando $0<\alpha<1 / 2$ temos ind $\left(c_{2}\right)=3$. 


\section{Capítulo 7}

\section{Exemplos de Katok Com Poucas Geodésicas Fechadas Curtas}

Nesse capítulo iremos generalizar a construção do Capítulo 5 para obter outros exemplos de métricas Finsler.

\subsection{Geodésica como ponto crítico de uma função}

Seja $(M, g)$ uma variedade Riemanniana tal que todas as geodésicas de $g$ são fechadas de período $2 \pi$ e seja $C^{*}=T_{1}^{*} M / S^{1}$ como na Definição 4.51. Então temos a $S^{1}$ fibração $S^{1} \rightarrow T_{1}^{*} M \stackrel{\pi}{\rightarrow} C^{*}$ induzida pelo fluxo co-geodésico em $T^{*} M$. Em $C^{*}$ temos a involução $\theta: C^{*} \rightarrow C^{*}$ que aplica cada geodésica fechada na mesma com direção oposta, isto é, se $c \in C^{*}$

$$
\theta(c)(t)=c(2 \pi-t) .
$$

Se $\omega$ é a 2-forma simplética canônica em $T^{*} M$ então existe uma 2-forma simplética $\widetilde{\omega}$ em $C$ tal que

$$
\pi^{*} \widetilde{\omega}=\left.\omega\right|_{T_{1}^{*} M}
$$

A involução $\theta$ induz uma involução $\bar{\theta}: T_{1}^{*} M \rightarrow T_{1}^{*} M$ tal que vale as igualdades:

$$
\bar{\theta}(x, \xi):=(x,-\xi) \quad \text { e } \quad \pi \circ \bar{\theta}=\theta \circ \pi .
$$

Afirmação 7.1. $\bar{\theta}^{*} \omega=-\omega$.

Demonstração. Se $(p, q)$ são coordenadas de $T^{*} M$ então $\omega=\sum d p_{i} \wedge d q_{i}$. Assim,

$$
\begin{aligned}
\overline{\boldsymbol{\theta}}^{*} \omega & =\omega(d \overline{\boldsymbol{\theta}}, d \overline{\boldsymbol{\theta}}) \\
& =\sum d p_{i}(d \overline{\boldsymbol{\theta}}) \wedge d q_{i}(d \overline{\boldsymbol{\theta}}) \\
& =\sum d p_{i} \wedge\left(-d q_{i}\right) \\
& =-\omega .
\end{aligned}
$$

Afirmação 7.2. $\theta^{*} \widetilde{\omega}=-\widetilde{\omega}$.

Demonstração. Pelas equações (7.3) e (7.2) temos $\bar{\theta}^{*} \pi^{*}=\pi^{*} \theta^{*}$ e

$$
\pi^{*} \theta^{*} \widetilde{\omega}=\bar{\theta}^{*} \pi^{*} \widetilde{\omega}=\bar{\theta}^{*} \omega=-\omega=-\pi^{*} \widetilde{\omega} .
$$

Assim,

$$
\theta^{*} \widetilde{\omega}=-\widetilde{\omega}
$$


Definição 7.3. Seja $\tilde{f}: C^{*} \rightarrow \mathbb{R}$ uma função $C^{\infty}$. Defina $f: T_{1}^{*} M \rightarrow \mathbb{R}$ por $f=\widetilde{f} \circ \pi$. Além disso, como para cada $(x, \eta) \in T^{*} M$ existe $\lambda>0$ e $\xi \in T_{1}^{*} M$ tal que $\eta=\lambda \xi$, então, defina $H_{1}: T^{*} M \rightarrow \mathbb{R}$ por

$$
H_{1}(x, \eta)=\lambda f(x, \xi) .
$$

Observação 7.4. Note que

$$
H_{1}(x, \mu \eta)=\mu H_{1}(x, \eta)
$$

para todo $(x, \eta) \in T^{*} M$ e $\mu>0$, isto é, $H_{1}$ é positivamente homogêneo de grau 1.

Seja $H_{0}$ como definido em (4.9). Daqui em diante iremos estudar hamiltonianos da forma

$$
H_{\alpha}=H_{0}+\alpha H_{1}
$$

Como vimos na Observação 5.3 a função $H_{\alpha}$, como definida acima, é uma co-norma Finsler se $\alpha$ é suficientemente pequeno. Logo, pelo Teorema 1.25 segue que

$$
F_{\alpha}=H_{\alpha} \circ \mathscr{L}_{\frac{1}{2} H_{\alpha}^{2}}^{-1}
$$

onde $\mathscr{L}_{\frac{1}{2} H_{\alpha}^{2}}: T^{*} M \rightarrow T M$ é a transformada de Legendre associada a função $\frac{1}{2} H_{\alpha}^{2}$, é uma métrica Finsler se $\alpha$ é suficientemente pequeno.

Proposição 7.5. A métrica Finsler $F_{\alpha}$ como definida em (7.4) é simétrica se, e somente se, $\widetilde{f}$ é invariante $\operatorname{sob} \theta$.

Demonstração. Primeiro mostraremos que $H_{1}$ é simétrica se, e somente se, $\widetilde{f}$ é invariante sob $\theta$. Se $\widetilde{f}$ é invariante $\operatorname{sob} \theta$ temos

$$
\tilde{f} \circ \theta=\widetilde{f} .
$$

Logo, se $(x, \xi) \in T_{1}^{*} M$, usando a Definição 7.3 e as equações (7.3) e (7.5) segue-se que

$$
\begin{aligned}
H_{1}(x,-\xi) & =H_{1}(\bar{\theta}(x, \xi)) \\
& =f \circ \bar{\theta}(x, \xi) \\
& =\widetilde{f} \circ \pi \circ \bar{\theta}(x, \xi) \\
& =\widetilde{f} \circ \theta \circ \pi(x, \xi) \\
& =\widetilde{f} \circ \pi(x, \xi) \\
& =H_{1}(x, \xi)
\end{aligned}
$$

Pela homogeneidade, concluímos que

$$
H_{1}(x,-\xi)=H_{1}(x, \xi), \text { para todo }(x, \xi) \in T^{*} M .
$$

Agora, se $H_{1}(x,-\eta)=H_{1}(x, \eta)$ para todo $(x, \eta) \in T^{*} M$ segue pela equação (7.3) que para todo $(x, \xi) \in$ $T_{1}^{*} M$

$$
\begin{aligned}
& \tilde{f} \circ \pi(x,-\xi)=\widetilde{f} \circ \pi(x, \xi) \\
\Rightarrow & \tilde{f} \circ \pi \circ \bar{\theta}(x, \xi)=\tilde{f} \circ \pi(x, \xi) \\
\Rightarrow & \widetilde{f} \circ \theta(\pi(x, \xi))=\widetilde{f}(\pi(x, \xi))
\end{aligned}
$$

Logo, como $\pi$ é sobrejetora temos

$$
\widetilde{f} \circ \theta=\widetilde{f} .
$$

Como $\mathscr{L}_{\frac{1}{2} H_{\alpha}^{2}}^{-1}$ é linear na direção da fibra, para que $F_{\alpha}$ seja simétrica é necessário apenas que $H_{\alpha}$ seja simé- 
trica. Assim, como

$$
\begin{aligned}
H_{\alpha}(x,-\xi) & =H_{0}(x,-\xi)+\alpha H_{1}(x,-\xi) \\
& =H_{0}(x, \xi)+\alpha H_{1}(x,-\xi)
\end{aligned}
$$

segue-se que $H_{\alpha}$ é simétrica se, e somente se, $H_{1}$ é simétrica. Portanto, $F_{\alpha}$ é simétrica se, e somente se, $\widetilde{f}$ é invariante $\operatorname{sob} \theta$.

A função $\widetilde{f}$ definida em $C^{*}$ induz um campo Hamiltoniano $X_{\widetilde{f}}$ com respeito a forma simplética $\widetilde{\omega}$ e denotaremos seu fluxo por $\psi_{t}^{\tilde{f}}$. Como

$$
\pi^{*} \widetilde{\omega}=\omega \quad \text { e }\left.\quad H_{1}\right|_{T_{1}^{*} M}=\widetilde{f} \circ \pi
$$

temos

$$
\begin{aligned}
\omega\left(X_{H_{1}}(x, \xi), \cdot\right) & =d H_{1}(x, \xi) \\
& =d(\widetilde{f} \circ \pi(x, \xi)) \\
& =d \widetilde{f}(\pi(x, \xi)) \cdot d \pi(x, \xi) \\
& =\widetilde{\omega}\left(X_{\widetilde{f}}(\pi(x, \xi)), d \pi(x, \xi)(\cdot)\right)
\end{aligned}
$$

Além disso, como

$$
\omega\left(X_{H_{1}}(x, \xi), \cdot\right)=\pi^{*} \widetilde{\omega}\left(X_{H_{1}}(x, \xi), \cdot\right)=\widetilde{\omega}\left(d \pi(x, \xi)\left(X_{H_{1}}(x, \xi)\right), d \pi(x, \xi)(\cdot)\right)
$$

obtemos

$$
\widetilde{\omega}\left(X_{\widetilde{f}}(\pi(x, \xi)), \cdot\right)=\widetilde{\omega}\left(d \pi(x, \xi)\left(X_{H_{1}}(x, \xi)\right), \cdot\right)
$$

ou seja, $X_{\widetilde{f}}(\pi(x, \xi))=d \pi(x, \xi)\left(X_{H_{1}}(x, \xi)\right)$ para todo $(x, \xi) \in T_{1}^{*} M$. Disso segue que

$$
\begin{aligned}
\left.\frac{d}{d t} \psi_{t}^{\widetilde{f}}(\pi(x, \xi))\right|_{t=0} & =X_{\widetilde{f}}(\pi(x, \xi)) \\
& =d \pi(x, \xi) X_{H_{1}}(x, \xi) \\
& =\left.\frac{d}{d t}\left(\pi \circ \psi_{t}^{H_{1}}(x, \xi)\right)\right|_{t=0}
\end{aligned}
$$

para todo $(x, \xi) \in T_{1}^{*} M$. Logo, pela unicidade de curvas integrais temos

$$
\psi_{t}^{\widetilde{f}}(\pi(x, \xi))=\pi \circ \psi_{t}^{H_{1}}(x, \xi) .
$$

Lema 7.6. A função $H_{1}: T^{*} M \rightarrow \mathbb{R}$ é constante ao longo das órbitas da $S^{1}$-ação em $T^{*} M$.

Demonstração. Lembre que uma órbita de $(x, \xi) \in T_{1}^{*} M$ pela $S^{1}$-ação é da forma

$$
s \cdot(x, \xi)=\left(\gamma_{x}^{v}(2 \pi s), g\left(\dot{\gamma}_{x}^{v}(2 \pi s), \cdot\right)\right), \quad s \in S^{1}
$$

onde $(x, \xi) \in T M$ é tal que $\xi=g(v, \cdot)$ e $\gamma_{x}^{v}$ é a geodésica em M tal que $\gamma_{x}^{v}(0)=x$ e $\dot{\gamma}_{x}^{v}(0)=v$. Assim, se para $(x, \xi) \in T_{1}^{*} M$ temos

$$
H_{1}(x, \xi)=\widetilde{f} \circ \pi(x, \xi)=\widetilde{f}\left(\gamma_{x}^{v}, g\left(\dot{\gamma}_{x}^{v}, \cdot\right)\right)=\mu
$$

então

$$
H_{1}(s \cdot(x, \xi))=\tilde{f} \circ \pi\left(\gamma_{x}^{v}(2 \pi s), g\left(\dot{\gamma}_{x}^{v}(2 \pi s), \cdot\right)\right)=\tilde{f}(\Gamma, g(\dot{\Gamma}, \cdot))
$$

onde $\Gamma$ é a geodésica em M tal que $\Gamma(0)=\gamma_{x}^{v}(2 \pi s)$ e $\dot{\Gamma}(0)=\dot{\gamma}_{x}^{v}(2 \pi s)$. Logo, pela unicidade das geodésicas 
temos

$$
\Gamma=\gamma_{x}^{v}
$$

Portanto, $H_{1}(s \cdot(x, \xi))=\mu$ para todo $s \in S^{1}$.

Lema 7.7. Os fluxos $\psi_{t}^{H_{1}}$ e $\psi_{t}^{H_{0}}$ comutam.

Demonstração. Pelo Lema 5.6 segue que as curvas integrais de $X_{H_{0}}$ são da forma $(\gamma(t), g(\dot{\gamma}(t), \cdot))$ onde $g(\dot{\gamma}(t), \dot{\gamma}(t))=1$, ou seja, são as órbitas da $S^{1}$-ação em $T_{1}^{*} M$. Assim, do Lema 7.6 segue-se que

$$
\left(H_{1}, H_{0}\right)(x, \xi)=\left.\frac{d}{d t} H_{1}\left(\psi_{t}^{H_{0}}\right)\right|_{t=0}=\left.\frac{d}{d t}(\text { constante })\right|_{t=0}=0 .
$$

e pelo Teorema 4.25 concluímos que $\psi_{t}^{H_{1}}$ e $\psi_{t}^{H_{0}}$ comutam.

O Teorema a seguir será necessário quando estivermos estudando as órbitas não triviais de $X_{\widetilde{f}}$. Ele é atribuído a D. Epstein apesar de não o ter publicado.

Teorema 7.8. Se $X$ é um campo de vetores $C^{1}$ em $\mathbb{R}^{n} \operatorname{com} X(0)=0$, então existe um $\varepsilon>0$ tal que todas as órbitas periódicas não triviais de $X$ em uma vizinhança de 0 tem período maior que $\varepsilon$.

Demonstração. Seja U uma vizinhança convexa de 0 e seja $r$ o máximo de $\|D X\|$ em U, isto é,

$$
r=\max _{x \in U}\|D X(x)\| .
$$

Se $c(t)$ é uma órbita periódica não trivial de $\mathrm{X}$ em $\mathrm{U}$, seja $d$ o diâmetro de $c$ e sejam $t_{1}, t_{2}$ tais que

$$
\left\|c\left(t_{2}\right)-c\left(t_{1}\right)\right\|=d .
$$

Então, $t_{1}$ e $t_{2}$ dividem $c$ em duas partes $c_{1}$ e $c_{2}$. Se $v$ é definido por

$$
v=\frac{c\left(t_{2}\right)-c\left(t_{1}\right)}{d}
$$

temos

$$
\begin{aligned}
\int_{c_{1}}\langle v, X(c(t))\rangle d t & =\left.\langle v, c(t)\rangle\right|_{t_{1}} ^{t_{2}} \\
& =\left\langle v, c\left(t_{2}\right)-c\left(t_{1}\right)\right\rangle \\
& =\frac{1}{d}\left\|c\left(t_{2}\right)-c\left(t_{1}\right)\right\|^{2} \\
& =d
\end{aligned}
$$

onde $\langle$,$\rangle é o produto interno usual do \mathbb{R}^{n}$. Pelo Teorema do Valor Médio para Integrais existe $t^{*} \in\left(t_{1}, t_{2}\right)$ tal que

$$
d=\int_{t_{1}}^{t_{2}}\langle v, X(c(t))\rangle d t=\left(t_{2}-t_{1}\right) \cdot\left\langle v, X\left(c\left(t^{*}\right)\right)\right\rangle \leq T \cdot \max _{t_{1} \leq t \leq t_{2}}\langle v, X(c(t))\rangle
$$

onde $T$ é o período de $c$. Logo, existe $t^{\prime}$ tal que

$$
\left\langle v, X\left(c\left(t^{\prime}\right)\right)\right\rangle \geq \frac{d}{T}
$$

De forma análoga temos

$$
\int_{c_{2}}\langle v, X(c(t))\rangle d t=-d
$$

e existe $t^{\prime \prime}$ tal que

$$
\left\langle v, X\left(c\left(t^{\prime \prime}\right)\right)\right\rangle \leq-\frac{d}{T}
$$


Isso implica que

$$
\left\langle v, X\left(c\left(t^{\prime}\right)\right)-X\left(c\left(t^{\prime \prime}\right)\right)\right\rangle \geq \frac{2 d}{T}
$$

Além disso, pelo Teorema do Valor Médio para funções vetoriais temos

$$
\left\|X\left(c\left(t^{\prime}\right)\right)-X\left(c\left(t^{\prime \prime}\right)\right)\right\| \leq r d
$$

Assim, das equações (7.7), (7.8) e pela desigualdade de Cauchy-Schwarz obtemos

$$
\begin{aligned}
\frac{2 d}{T} & \leq\left\langle v, X\left(c\left(t^{\prime}\right)\right)-X\left(c\left(t^{\prime \prime}\right)\right)\right\rangle \\
& \leq\|v\| \cdot\left\|X\left(c\left(t^{\prime}\right)\right)-X\left(c\left(t^{\prime \prime}\right)\right)\right\| \\
& \leq r d
\end{aligned}
$$

Portanto,

$$
T \geq \frac{2}{r}
$$

e fazendo $\varepsilon=2 / r$ obtemos o desejado.

Teorema 7.9. Seja $F_{\alpha}$ uma métrica Finsler como em (7.4). Cada ponto crítico de $\widetilde{f}$ da origem a uma geodésica fechada cujo comprimento vai para $2 \pi$ quando $\alpha \rightarrow 0$. O comprimento de todas as outras geodésicas fechadas vão para $\infty$ quando $\alpha \rightarrow 0$.

Demonstração. Como $\psi_{t}^{H_{0}}$ e $\psi_{t}^{H_{1}}$ comutam (Lema 7.7) temos

$$
\psi_{t}^{H_{\alpha}}=\psi_{t}^{H_{0}} \circ \psi_{\alpha t}^{H_{1}} .
$$

Assim, se $\eta \in T_{1}^{*} M$ é um ponto periódico de $\psi_{t}^{H_{\alpha}}$ de período $T$, então,

$$
\psi_{\alpha T}^{H_{1}} \eta=\psi_{-T}^{H_{1}} \eta
$$

e $\widetilde{\eta}=\pi(\eta)$ é um ponto periódico de $\psi_{t}^{\widetilde{f}}$ de período $\alpha T$ pois por (7.6) e da definição de $\pi$ temos

$$
\psi_{\alpha T}^{\widetilde{f}} \widetilde{\eta}=\pi \circ \psi_{\alpha T}^{H_{1}} \eta=\pi \circ \psi_{-T}^{H_{0}} \eta=\pi(\eta)=\widetilde{\eta} .
$$

Logo, existem duas possibilidades:

1. $\psi_{t}^{\widetilde{f}} \widetilde{\eta}$ é uma órbita periódica trivial, isto é, $\psi_{t}^{\widetilde{f}} \widetilde{\eta}=\widetilde{\eta}$ para todo $t \in \mathbb{R}$. Assim, como

$$
X_{\widetilde{f}}(\widetilde{\eta})=\left.\frac{d}{d t} \psi_{t}^{\widetilde{f}} \widetilde{\eta}\right|_{t=0}=\left.\frac{d \widetilde{\eta}}{d t}\right|_{t=0}=0
$$

segue que

$$
d \widetilde{f}(\widetilde{\eta})=\widetilde{\omega}\left(X_{\widetilde{f}}(\widetilde{\eta}), \cdot\right) \equiv 0,
$$

ou seja, $\widetilde{\eta}$ é um ponto crítico de $\widetilde{f}$.

2. $\psi_{t}^{\widetilde{f}} \widetilde{\eta}$ é uma órbita periódica não trivial de $X_{\widetilde{f}}$ e $\alpha T$ é um múltiplo do seu período.

Primeiro examinaremos o caso em que $\widetilde{\eta}$ é um ponto crítico de $\widetilde{f}$. Nesse caso, pela equação (7.6) obtemos

$$
\pi\left(\psi_{t}^{H_{1}} \eta\right)=\psi_{t}^{\widetilde{f}} \widetilde{\eta}=\widetilde{\eta}=\pi(\eta)
$$

Logo, $\psi_{t}^{H_{1}} \eta$ é um ponto na $S^{1}$-órbita de $\eta$. Disso segue que

$$
\psi_{t}^{H_{\alpha}} \eta=\psi_{t}^{H_{0}} \circ \psi_{\alpha t}^{H_{1}} \eta \in S^{1} \cdot \eta
$$


para todo $t \in S^{1}$. Então, $\eta$ é um ponto periódico de $X_{H_{\alpha}}$. Para determinar o seu período, introduziremos um sistema de coordenadas simplético $\left(p_{i}, q_{i}\right)$ em $T^{*} M$ tal que $p_{1}=t$ é o parâmetro do tempo ao longo de $\psi_{t}^{H_{0}} \eta$ e tal que $q_{1}(\eta)=H_{0}(\eta)$. Assim, como para todo $\eta \in T^{*} M$ existem $\lambda>0$ e $\rho \in T_{1}^{*} M$ tais que $\eta=\lambda \rho$ e como

$$
H_{0}(\eta)=H_{0}(\lambda \rho)=\lambda H_{0}(\rho)=\lambda
$$

obtemos

$$
H_{1}(\eta)=\lambda H_{1}(\rho)=H_{0}(\eta) \cdot \tilde{f} \circ \pi(\rho)=q_{1}(\eta) \cdot \tilde{f} \circ \pi(\rho) .
$$

Como $p_{1}=t$ é o parâmetro de tempo ao longo de $\psi_{t}^{H_{0}} \eta$ segue que $\pi(\rho)$ não depende de $p_{1}$, além disso, como $H_{0}$ é constante ao longo da órbita de qualquer ponto em $T^{*} M$ segue que $\pi(\rho)$ também não depende de $q_{1}$. Logo,

$$
H_{1}(\eta)=q_{1}(\eta) \cdot \widetilde{f}\left(p_{2}, \ldots, p_{n}, q_{2}, \ldots, q_{n}\right)
$$

isto é, $\widetilde{f}$ não depende das coordenadas $p_{1}, q_{1}$, e assim,

$$
H_{\alpha}(\eta)=q_{1}(\eta)+\alpha q_{1}(\eta) \cdot \widetilde{f}\left(p_{2}, \ldots, p_{n}, q_{2}, \ldots, q_{n}\right) .
$$

As equações de Hamilton para $H_{\alpha}$ são então:

$$
\dot{p}_{1}=\dot{t}=\frac{\partial H_{\alpha}}{\partial q_{1}}=1+\alpha \widetilde{f}, \quad \dot{q}_{1}=-\frac{\partial H_{\alpha}}{\partial p_{1}}=0
$$

$\mathrm{e}$

$$
\dot{p}_{i}=\frac{\partial H_{\alpha}}{\partial q_{i}}=\alpha q_{1} \frac{\partial \widetilde{f}}{\partial q_{i}}, \quad \dot{q}_{i}=-\frac{\partial H_{\alpha}}{\partial p_{i}}=-\alpha q_{1} \frac{\partial \widetilde{f}}{\partial p_{i}}
$$

para $i>1$. Como $q_{1}(\eta)=1$ se $\eta \in T_{1}^{*} M$ e $\dot{t}=1+\alpha \widetilde{f}$ é a variação do parâmetro $p_{1}=t$ ao longo de $\psi_{t}^{H_{0}} \eta$ temos

$$
t=(1+\alpha \widetilde{f}(\widetilde{\eta})) s
$$

onde $s$ é o parâmetro de tempo ao longo de $\psi_{t}^{H_{\alpha}} \eta$. Assim, se o período de $\psi_{t}^{H_{\alpha}} \eta$ é $T$, então, como o período de $\psi_{t}^{H_{0}} \eta$ é $2 \pi$ temos

$$
2 \pi=(1+\alpha \widetilde{f}(\widetilde{\eta})) T .
$$

Portanto, $2 \pi /(1+\alpha \widetilde{f}(\widetilde{\eta}))$ é o período de $\psi_{t}^{H_{\alpha}} \eta$.

Para examinar as órbitas periódicas de $H_{\alpha}$ vindo de órbitas periódicas não triviais de $X_{\widetilde{f}}$ podemos aplicar o Teorema 7.8 ao campo de vetores $X_{\widetilde{f}}$ em $C^{*}$. Como $C^{*}$ é compacto existe uma cota inferior para o período de qualquer órbita periódica não trivial de $X_{\widetilde{f}}$. Além disso, como $X_{\widetilde{f}}$ é um campo de vetores $C^{\infty}$ tal que $c_{0} \equiv$ constante $\in C^{*}$ e $X_{\widetilde{f}}\left(c_{0}\right)=0$, então, existe $\varepsilon>0$ tal que toda órbita periódica não trivial na vizinhança de $c_{0}$ tem período maior que $\varepsilon$. Como $\alpha T$ é um múltiplo do período $L$ de $X_{\widetilde{f}}$, isto é, $\alpha T=m L$ para algum $m \in \mathbb{R}$, concluímos que

$$
\frac{\alpha T}{m}=L>\varepsilon \Longrightarrow T>\frac{\varepsilon m}{\alpha} .
$$

Portanto, quando $\alpha \rightarrow 0$ o comprimento das órbitas de $X_{H_{\alpha}}$ tendem a $\infty$.

Teorema 7.10. Se $M=S^{n}, \mathbb{C} P^{n}, \mathbb{H} P^{n}$ e g é a métrica Riemanniana canônica dessas variedades então para qualquer $\varepsilon>0$ existe uma métrica Finsler em $M$ que está $\varepsilon$ próxima de g com $\operatorname{dim} M$ geodésicas fechadas com comprimento em $(2 \pi-\varepsilon, 2 \pi+\varepsilon)$ e tal que o comprimento de todas as outras geodésicas fechadas é maior que $1 / \varepsilon$.

Demonstração. Como M é uma variedade Riemanniana tal que todas as geodésicas são fechadas de mesmo comprimento $2 \pi$ então $C^{*}$ é simplesmente conexa. Assim, pelo Teorema 5.1 em [Tak68] segue que existe uma função em $C^{*}$ com somente $\operatorname{dim} M$ pontos críticos se $\operatorname{dim} C^{*} \geq 6$. Mas se $\operatorname{dim} C^{*}<6$ temos $M=S^{2}, S^{3}$ ou $M=\mathbb{C} P^{1}=S^{2}$ e então $C^{*}=S^{2}$ ou $C^{*}=S^{2} \times S^{2}$ (ver Proposição 2.9 em [Bes12]). E também nesses casos existe uma função em $C^{*} \operatorname{com} \operatorname{dim} M$ pontos críticos. Portanto, pelo Teorema 7.9 concluímos o desejado. 


\subsection{Métricas Finsler Simétricas}

Iremos examinar, nessa seção, a quantidade mínima de geodésicas em $M=S^{n}$ no caso em que as métricas Finsler são simétricas. Os métodos de perturbação em [Wei78] e na Seção 4.6 ou a teoria de LusternikSchnirelmann implicam que qualquer métrica Finsler suficientemente próxima da métrica Riemanniana canônica definida em $\mathrm{M}$ tem pelo menos tantas geodésicas fechadas de comprimento próximo de $2 \pi$ quanto uma função em $C^{*} / \theta$ tem pontos críticos. Aqui, $\theta$ é a aplicação $c \mapsto-c$ definida em (7.1).

Por outro lado, se temos uma função em $C^{*} / \theta$ com $k$ pontos críticos, então ela pode ser levantada para uma função em $C^{*} \operatorname{com} 2 k$ pontos críticos e pelo Teorema 7.9 segue que a correspondente métrica Finsler simétrica $F_{\alpha}$ tem $k$ geodésicas fechadas de comprimento próximo de $2 \pi$, uma vez que contamos $c(t)$ e $c(-t)$ como uma única geodésica fechada agora, e o comprimento de todas as outras geodésicas fechadas se tornam arbitrariamente grandes quando $\alpha$ se aproxima de zero. Mas o problema de encontrar um número mínimo de pontos críticos para uma função em $C^{*} / \theta$ ainda está em aberto.

Definição 7.11. As variedades Grassmanianas reais $G_{n, k}^{0}$ e $G_{n, k}$ são definidas, respectivamente, como a variedade de $n$-planos orientáveis e não orientáveis em $\mathbb{R}^{n+k}$ passando pela origem.

Observação 7.12. A dimensão de $G_{n, k}$ é $n k$.

Como $C^{*}=T_{1}^{*} S^{n} / S^{1}$ é o espaço das geodésicas de $S^{n}$ na métrica riemanniana canônica de curvatura constante (vistas em $T_{1}^{*} S^{n}$ ) e podemos identificar cada geodésica em $S^{n}$ com o plano de $\mathbb{R}^{n+1}$, que passa pela origem, que a contém, então, $C^{*}$ pode ser identificado com $G_{2, n-1}^{0}$ e $C^{*} / \theta \operatorname{com} G_{2, n-1}$.

Teorema 7.13. Existem métricas Finsler Simétricas em $S^{n}$ em qualquer vizinhança da métrica de curvatura constante, com somente $2 n-1$ geodésicas fechadas de comprimento próximo de $2 \pi$ e tal que o comprimento de todas as outras geodésicas fechadas é maior que qualquer número prescrito.

Demonstração. Pela Proposição 2.9 e uma observação abaixo dessa proposição em [Tak68] vemos que toda variedade compacta e conexa $\mathrm{M}$ admite uma função $\operatorname{com} \operatorname{dim} M+1$ pontos críticos. Então, como $\operatorname{dim} G_{2, n-1}=2(n-1)$ segue que existe uma função em $G_{2, n-1} \operatorname{com} 2 n-1$ pontos críticos. Pela identificação de $G_{2, n-1} \operatorname{com} C^{*} / \theta$ segue que também existe uma função em $C^{*} / \theta \operatorname{com} 2 n-1$ pontos críticos.

Portanto, pelo Teorema 7.9 e a discussão que fizemos no início dessa seção segue que a correspondente métrica Finsler $F_{\alpha}$ possui $2 n-1$ geodésicas de comprimento próximo de $2 \pi$ se $\alpha$ é suficientemente pequeno e o comprimento de todas as outras geodésicas vão para $\infty$ quando $\alpha \rightarrow 0$.

A Proposição 2.9 em [Tak68] nos dá um limitante inferior para o número mínimo de pontos críticos que uma função suave definida em $G_{2, n-1}$ pode ter. No entanto, para $n=3$, J. Milnor deu um exemplo de uma função em $G_{2,2}$ com somente 4 pontos críticos, que é uma quantidade menor do que a obtida através dessa proposição. O próprio Milnor não publicou esse exemplo, mas ele é descrito no artigo [Zil83], no qual se baseia esse trabalho. Esse exemplo, é uma peça fundamental na demonstração de que em $S^{3}$ existem métricas Finsler com somente 4 geodésicas fechadas de comprimento próximo de $2 \pi$. Mas antes da demonstração desse resultado devemos ter em mente o seguinte:

Definição 7.14. Seja $V$ um espaço vetorial sobre um corpo K. Dado $x \in \wedge^{n} V$, dizemos que $x$ é decomponível se existem $v_{1}, \ldots, v_{n} \in V$ tais que $x=v_{1} \wedge \cdots \wedge v_{n}$.

Proposição 7.15. O produto exterior $u_{1} \wedge \cdots \wedge u_{p}$ de $p$ vetores $u_{i} \in V$ se anula se, e somente se, os vetores são linearmente dependentes.

Demonstração. Se existe uma relação linear

$$
\lambda_{1} u_{1}+\cdots+\lambda_{p} u_{p}=0
$$

com $\lambda_{i} \neq 0$ então $u_{i}$ é uma combinação linear dos outros vetores

$$
u_{i}=\sum_{j \neq i} \mu_{j} u_{j}
$$


onde $\mu_{j}=\lambda_{j} / \lambda_{i}$. Assim,

$$
u_{1} \wedge \cdots \wedge u_{p}=u_{1} \wedge \cdots \wedge\left(\sum_{j \neq i} \mu_{j} u_{j}\right) \wedge u_{i+1} \wedge \cdots \wedge u_{p}
$$

e expandindo a expressão acima segue que cada termo da soma tem uma variável repetida $u_{j}$ e, portanto, se anula.

Para a recíproca, sejam $u_{1}, \ldots, u_{p}$ vetores em $\mathrm{V}$ tais que $u_{1} \wedge \cdots \wedge u_{p}=0$. Se $u_{1}, \ldots, u_{p}$ são vetores linearmente independentes podemos estendê-los a uma base de $\mathrm{V}$ e isso implica que $u_{1} \wedge \cdots \wedge u_{p}$ é um vetor da base de $\wedge^{p} V$ e assim é não nulo, o que gera uma contradição. Portanto, se $u_{1} \wedge \cdots \wedge u_{p}=0$ então $u_{1}, \ldots, u_{p}$ são vetores linearmente dependentes.

Lema 7.16. Seja $x \in \wedge^{2} V$ um elemento não nulo. Então $x$ é decomponível se, e somente se, $x \wedge x=0 \in \wedge^{4} V$.

Demonstração. Se $x$ é decomponível, então existem vetores $v_{1}, v_{2} \in V$ tais que $x=v_{1} \wedge v_{2}$, então

$$
x \wedge x=v_{1} \wedge v_{2} \wedge v_{1} \wedge v_{2}=-v_{1} \wedge v_{2} \wedge v_{2} \wedge v_{1}=0 .
$$

Provaremos a recíproca por indução na dimensão de V. Se $\operatorname{dim} V=0,1$ então $\wedge^{2} V=0$, assim o primeiro caso é $\operatorname{dim} V=2$. Nesse caso $\operatorname{dim} \wedge^{2} V=1$ e $v_{1} \wedge v_{2}$ é um elemento não nulo se $v_{1}, v_{2}$ é uma base de $\mathrm{V}$, então todo elemento não nulo de $\wedge^{2} V$ é decomponível.

Consideraremos o caso $\operatorname{dim} V=3$ separadamente. Dado $x \in \wedge^{2} V$ não nulo, defina $f_{x}: V \rightarrow \wedge^{3} V$ por

$$
f_{x}(v)=x \wedge v
$$

Como $\operatorname{dim} \wedge^{3} V=1$ segue que $\operatorname{dim} \operatorname{ker} f_{x} \geq 2$. Assim, sejam $u_{1}, u_{2}$ vetores linearmente independentes em $\operatorname{ker} f_{x}$ e estenda para uma base $u_{1}, u_{2}, u_{3}$ de V. Dessa forma, existem escalares $\lambda_{1}, \lambda_{2}, \lambda_{2}$ tais que

$$
x=\lambda_{1} u_{2} \wedge u_{3}+\lambda_{2} u_{1} \wedge u_{3}+\lambda_{3} u_{1} \wedge u_{2} .
$$

Como $u_{1} \in \operatorname{ker} f_{x}$ temos

$$
0=x \wedge u_{1}=\lambda_{1} u_{2} \wedge u_{3} \wedge u_{1}
$$

e isto implica que $\lambda_{1}=0$. Similarmente temos

$$
0=x \wedge u_{2} \Longrightarrow \lambda_{2}=0 .
$$

Logo, $x=\lambda_{3} u_{1} \wedge u_{2}$, isto é, $x$ é decomponível.

Agora assuma que o lema é verdadeiro se $\operatorname{dim} V \leq n-1$ e considere o caso $\operatorname{dim} V=n$. Seja $v_{1}, \ldots, v_{n}$ uma base ordenada de $\mathrm{V}$ e escreva

$$
\begin{aligned}
x & =\sum_{1 \leq i<j}^{n} a_{i j} v_{i} \wedge v_{j} \\
& =\left(\sum_{i=1}^{n-1} a_{i n} v_{i}\right) \wedge v_{n}+\sum_{1 \leq i<j}^{n-1} a_{i j} v_{i} \wedge v_{j} \\
& =u \wedge v_{n}+x^{\prime}
\end{aligned}
$$

onde $u \in U, x^{\prime} \in \wedge^{2} U$ e $U$ é o espaço gerado por $v_{1}, \ldots, v_{n-1}$. Como estamos assumindo que $x \wedge x=0$ temos

$$
0=x \wedge x=\left(u \wedge v_{n}+x^{\prime}\right) \wedge\left(u \wedge v_{n}+x^{\prime}\right)=2 x^{\prime} \wedge u \wedge v_{n}+x^{\prime} \wedge x^{\prime}
$$

Observe que $v_{3}$ não aparece na expressão de $u \wedge x^{\prime}$ ou $x^{\prime} \wedge x^{\prime}$ então obtemos

$$
u \wedge x^{\prime}=0 \quad \text { e } \quad x^{\prime} \wedge x^{\prime}=0 .
$$

Por hipótese de indução $x^{\prime} \wedge x^{\prime}=0$ implica que existem $u_{1}, u_{2} \in U$ tais que $x^{\prime}=u_{1} \wedge u_{2}$. Assim, da primeira 
equação em (7.11) temos

$$
u \wedge u_{1} \wedge u_{2}=0
$$

e pela Proposição 7.15 segue que

$$
\lambda u+\mu_{1} u_{1}+\mu_{2} u_{2}=0
$$

onde os coeficientes $\lambda, \mu_{1}, \mu_{2}$ não são todos nulos. Então, considere os seguintes casos:

1. Se $\lambda=0$, então, $u_{1}$ e $u_{2}$ são linearmente dependentes e, assim, $x^{\prime}=u_{1} \wedge u_{2}=0$. Disso, obtemos

$$
x=u \wedge v_{n} .
$$

2. Se $\lambda \neq 0$, obtemos

$$
u=-\frac{\mu_{1}}{\lambda} u_{1}-\frac{\mu_{2}}{\lambda} u_{2}
$$

e se $\lambda_{1}=-\mu_{1} / \lambda$ e $\lambda_{2}=-\mu_{2} / \lambda$ segue que

$$
x=\lambda_{1} u_{1} \wedge v_{n}+\lambda_{2} u_{2} \wedge v_{n}+u_{1} \wedge u_{2} .
$$

Como a equação (7.12) é um caso tri-dimensional que já mostramos ser decomponível, segue o desejado.

Portanto, em ambos os casos vemos que $x$ é decomponível.

Definição 7.17. Sejam $\mathrm{V}$ um espaço vetorial real e $\mathscr{C}=\left\{x_{1}, \ldots, x_{n}\right\}$ uma base para V. Se $v=a_{1} x_{1}+\cdots+$ $a_{n} x_{n} \in V$, definimos a norma de $v$ em relação a base $\mathscr{C},\|v\|_{\mathscr{C}}$ por:

$$
\|v\|_{\mathscr{C}}=\sqrt{a_{1}^{2}+\cdots+a_{n}^{2}} .
$$

Teorema 7.18. Em $S^{3}$ existem métricas Finsler (simétricas) com somente 4 geodésicas fechadas de comprimento próximo de $2 \pi$.

Demonstração. Primeiro observe que se $P \subset \mathbb{R}^{4}$ é um plano gerado pelos vetores $v_{1}, v_{2}$, podemos associar a $P$ o vetor

$$
\lambda=v_{1} \wedge v_{2} \in \wedge^{2} \mathbb{R}^{4} .
$$

O vetor $\lambda$ está unicamente determinado (a menos de multiplicação por escalar) por $P$ pois se escolhermos uma base diferente para $P$ que respeite a orientação, isto é, outra base positiva segue que o vetor $\widetilde{\lambda}$ correspondente será simplesmente $\lambda$ multiplicado pelo determinante da matriz de mudança de base. Podemos supor ainda, sem perda de geralidade, que $v_{1} \wedge v_{2}$ é unitário (em relação a uma base $\mathscr{C}$ de $\wedge^{2} \mathbb{R}^{4}$ que será construída abaixo). Disso segue que $G_{2,2}^{0}$ se identifica com o conjunto dos vetores unitários e decomponíveis de $\wedge^{2} \mathbb{R}^{4}$.

Tome agora o operador estrela de Hodge $\star: \wedge^{2} \mathbb{R}^{4} \rightarrow \wedge^{2} \mathbb{R}^{4}$ onde dada uma base ortonormal ordenada $e_{1}, e_{2}, e_{3}, e_{4}$ de $\mathbb{R}^{4}$ (dotado com o produto interno canônico) e uma permutação par $\left(i_{1}, i_{2}, i_{3}, i_{4}\right)$ de $\{1,2,3,4\}$ definimos

$$
\star\left(e_{i_{1}} \wedge e_{i_{2}}\right)=e_{i_{3}} \wedge e_{i_{4}} .
$$

Aplicando $\star$ na base canônica $e_{1} \wedge e_{2}, e_{1} \wedge e_{3}, e_{1} \wedge e_{4}, e_{2} \wedge e_{3}, e_{2} \wedge e_{4}, e_{3} \wedge e_{4}$ de $\wedge^{2} \mathbb{R}^{4}$ obtemos

$$
\begin{array}{ll}
\star\left(e_{1} \wedge e_{2}\right)=e_{3} \wedge e_{4}, & \star\left(e_{2} \wedge e_{3}\right)=e_{1} \wedge e_{4} \\
\star\left(e_{1} \wedge e_{3}\right)=-e_{2} \wedge e_{4}, & \star\left(e_{2} \wedge e_{4}\right)=-e_{1} \wedge e_{3} \\
\star\left(e_{1} \wedge e_{4}\right)=e_{2} \wedge e_{3}, & \star\left(e_{3} \wedge e_{4}\right)=e_{1} \wedge e_{2}
\end{array}
$$

e concluímos que $\star^{2}=$ Id. Logo, o polinômio característico de $\star$ é $x^{2}-1 \mathrm{e} \pm 1$ são os seus autovalores. Assim, $\star$ induz uma decomposição do espaço $\wedge^{2} \mathbb{R}^{4}$ dada por

$$
\wedge^{2} \mathbb{R}^{4}=\wedge^{+} \oplus \wedge^{-}
$$


onde $\wedge^{+} \mathrm{e} \wedge^{-}$são os autoespaços associados aos autovalores $1 \mathrm{e}-1$, respectivamente. Como $\mathscr{B}=\left\{e_{1} \wedge e_{2}+\right.$ $\left.e_{3} \wedge e_{4}, e_{1} \wedge e_{4}+e_{2} \wedge e_{3}, e_{2} \wedge e_{4}-e_{1} \wedge e_{3}\right\}$ gera $\wedge^{+} \mathrm{e} \mathscr{B}^{\prime}=\left\{e_{1} \wedge e_{3}+e_{2} \wedge e_{4}, e_{1} \wedge e_{2}-e_{3} \wedge e_{4}, e_{1} \wedge e_{4}-e_{2} \wedge e_{3}\right\}$ gera $\wedge^{-}$segue que $\mathscr{C}=\mathscr{B} \cup \mathscr{B}^{\prime}$ gera $\wedge^{2} \mathbb{R}^{4}$.

Assim, se $\omega \in \wedge^{2} \mathbb{R}^{4}$ é um vetor decomponível e unitário em relação a base $\mathscr{C}$, existem vetores $\omega^{+} \in \wedge^{+}$, $\omega^{-} \in \wedge^{-}$e escalares $a_{1}, a_{2}, a_{3}, b_{1}, b_{2}, b_{3}$ tais que $\omega=\omega^{+}+\omega^{-}$,

$$
\begin{aligned}
& \omega^{+}=a_{1}\left(e_{1} \wedge e_{2}+e_{3} \wedge e_{4}\right)+a_{2}\left(e_{1} \wedge e_{4}+e_{2} \wedge e_{3}\right)+a_{3}\left(e_{2} \wedge e_{4}-e_{1} \wedge e_{3}\right) \\
& \omega^{-}=b_{1}\left(e_{1} \wedge e_{3}+e_{2} \wedge e_{4}\right)+b_{2}\left(e_{1} \wedge e_{2}-e_{3} \wedge e_{4}\right)+b_{3}\left(e_{1} \wedge e_{4}-e_{2} \wedge e_{3}\right)
\end{aligned}
$$

$\mathrm{e}$

$$
a_{1}^{2}+a_{2}^{2}+a_{3}^{2}+b_{1}^{2}+b_{2}^{2}+b_{3}^{2}=1 .
$$

Além disso, como um vetor $u \in \wedge^{2} \mathbb{R}^{4}$ é decomponível se, somente se, $u \wedge u=0$ segue que

$$
0=\omega \wedge \omega=\left(\omega^{+}+\omega^{-}\right) \wedge\left(\omega^{+}+\omega^{-}\right)=\left(2 a_{1}^{2}+2 a_{2}^{2}+2 a_{3}^{2}-2 b_{1}^{2}-2 b_{2}^{2}-2 b_{3}^{2}\right) e_{1} \wedge e_{2} \wedge e_{3} \wedge e_{4} .
$$

Então,

$$
a_{1}^{2}+a_{2}^{2}+a_{3}^{2}=b_{1}^{2}+b_{2}^{2}+b_{3}^{2}
$$

e portanto, $\omega$ é um vetor decomponível e unitário (em relação a base $\mathscr{C}$ ) se, e somente se,

$$
a_{1}^{2}+a_{2}^{2}+a_{3}^{2}=b_{1}^{2}+b_{2}^{2}+b_{3}^{2}=\frac{1}{2} \Longleftrightarrow\left\|\omega^{+}\right\|_{\mathscr{C}}=\left\|\omega^{-}\right\|_{\mathscr{C}}=\frac{1}{\sqrt{2}} .
$$

Disso segue que o conjunto dos vetores unitários e decomponíveis de $\wedge^{2} \mathbb{R}^{4}$ se identifica com o espaço $S^{2} \times S^{2}$. Portanto,

$$
C^{*} \simeq G_{2,2}^{0} \simeq S^{2} \times S^{2}
$$

$\mathrm{e}$

$$
C^{*} / \theta \simeq G_{2,2} \simeq S^{2} \times S^{2} /(x, y) \sim(-x,-y) .
$$

Se escolhermos um sistema de coordenadas $\left(x_{i}, y_{i}\right), i=1,2,3$ em $S^{2} \times S^{2}$ onde $\sum x_{i}^{2}=\sum y_{i}^{2}=1$ então

$$
f\left(x_{i}, y_{i}\right)=f_{1}\left(x_{i}, y_{i}\right)+f_{2}\left(x_{i}, y_{i}\right)
$$

onde

$$
f_{1}\left(x_{i}, y_{i}\right)=\sum_{i=1}^{3}\left(\frac{y_{i}-x_{i}}{2}\right)^{2} \quad \text { e } \quad f_{2}\left(x_{i}, y_{i}\right)=x_{1}^{2}-x_{2}^{2}
$$

define uma função em $S^{2} \times S^{2}$ invariante sob $(x, y) \mapsto(-x,-y)$. Afirmo que a função induzida $\widetilde{f}$ em $S^{2} \times$ $S^{2} /(x, y) \sim(-x,-y)$ dada por

$$
\widetilde{f}\left(\left[\left(x_{i}, y_{i}\right)\right]\right)=f\left(x_{i}, y_{i}\right)
$$

tem 6 pontos críticos com 4 níveis críticos. De fato, tomando a parametrização de $S^{2} \times S^{2}$ da forma:

$$
\begin{array}{ll}
x_{1}=u_{1} & y_{1}=v_{1} \\
x_{2}=u_{2} & y_{2}=v_{2} \\
x_{3}=\sqrt{1-u_{1}^{2}-u_{2}^{2}} & y_{3}=\sqrt{1-v_{1}^{2}-v_{2}^{2}}
\end{array}
$$

obtemos

$$
f_{1}\left(u_{i}, v_{i}\right)=\left(\frac{v_{1}-u_{1}}{2}\right)^{2}+\left(\frac{v_{2}-u_{2}}{2}\right)^{2}+\left(\frac{\sqrt{1-v_{1}^{2}-v_{2}^{2}}-\sqrt{1-u_{1}^{2}-u_{2}^{2}}}{2}\right)^{2} .
$$

Logo,

$$
\nabla f_{1}\left(u_{i}, v_{i}\right)=\left(\frac{u_{1}}{2} \sqrt{\frac{1-v_{1}^{2}-v_{2}^{2}}{1-u_{1}^{2}-u_{2}^{2}}}-\frac{y_{1}}{2}, \quad \frac{u_{2}}{2} \sqrt{\frac{1-v_{1}^{2}-v_{2}^{2}}{1-u_{1}^{2}-u_{2}^{2}}}-\frac{y_{2}}{2}, \quad \frac{v_{1}}{2} \sqrt{\frac{1-u_{1}^{2}-u_{2}^{2}}{1-v_{1}^{2}-v_{2}^{2}}}-\frac{u_{1}}{2}, \quad \frac{v_{2}}{2} \sqrt{\frac{1-u_{1}^{2}-u_{2}^{2}}{1-v_{1}^{2}-v_{2}^{2}}}-\frac{u_{2}}{2}\right)
$$


$\mathrm{e}$

$$
\nabla f_{2}\left(u_{i}, v_{i}\right)=\left(\begin{array}{llll}
2 u_{1}, & -2 u_{2}, \quad 0,0
\end{array}\right) .
$$

Podemos ver que não existe nenhum ponto nessa parametrização (assim como nas outras parametrizações de $S^{2} \times S^{2}$ ) onde $\nabla f_{1}\left(u_{i}, v_{i}\right)=-\nabla f_{2}\left(u_{i}, v_{i}\right) \neq 0$. Então, os pontos críticos de $\widetilde{f}_{1}+\widetilde{f}_{2}$ são pontos críticos, simultaneamente, de $\widetilde{f}_{1}$ e $\widetilde{f}_{2}$.

Afirmo que $\nabla f_{1}\left(u_{i}, v_{i}\right)=0$ nos pontos de $\left(u_{i}, v_{i}\right) \mapsto\left(x_{j}, y_{j}\right)$ tais que $x_{j}=y_{j}$. De fato, pela equação (7.16) segue que $\nabla f_{1}\left(u_{i}, v_{i}\right)=0$ se, e somente se,

$$
\left\{\begin{array}{l}
v_{1}=u_{1} \sqrt{\frac{1-v_{1}^{2}-v_{2}^{2}}{1-u_{1}^{2}-u_{2}^{2}}} \\
v_{2}=u_{2} \sqrt{\frac{1-v_{1}^{2}-v_{2}^{2}}{1-u_{1}^{2}-u_{2}^{2}}}
\end{array}\right.
$$

- Se $u_{1}=0$ então de (I) segue que $v_{1}=0$ e de (II) temos

$$
v_{2}=u_{2} \sqrt{\frac{1-v_{2}^{2}}{1-u_{2}^{2}}} \Rightarrow v_{2}^{2}\left(1-u_{2}^{2}\right)=u_{2}^{2}\left(1-v_{2}^{2}\right) \Rightarrow v_{2}^{2}-v_{2}^{2} u_{2}^{2}=u_{2}^{2}-u_{2}^{2} v_{2}^{2} \Rightarrow v_{2}^{2}=u_{2}^{2}
$$

Como $\sqrt{\frac{1-v_{1}^{2}-v_{2}^{2}}{1-u_{1}^{2}-u_{2}^{2}}}>0$ vemos na equação (II) que $v_{2}$ e $u_{2}$ tem mesmo sinal. Então de (7.17) concluímos que $u_{2}=v_{2}$. E assim, pelas equações em (7.15) segue que

$$
x_{1}=x_{2}=0, x_{2}=y_{2} \text { e } x_{3}=y_{3} .
$$

- Seja $u_{1} \neq 0$. Então da equação (I) temos $v_{1} \neq 0$ e

$$
v_{1}^{2}=u_{1}^{2} \frac{1-v_{1}^{2}-v_{2}^{2}}{1-u_{1}^{2}-u_{2}^{2}} \Rightarrow v_{1}^{2}-v_{1}^{2} u_{1}^{2}-v_{1}^{2} u_{2}^{2}=u_{1}^{2}-u_{1}^{2} v_{1}^{2}-u_{1}^{2} v_{2}^{2}
$$

ou seja,

$$
v_{1}^{2}\left(1-u_{2}^{2}\right)=u_{1}^{2}\left(1-v_{2}^{2}\right) .
$$

Da equação (II) segue, de forma semelhante, que

$$
v_{2}^{2}\left(1-u_{1}^{2}\right)=u_{2}^{2}\left(1-v_{1}^{2}\right) .
$$

Assim,

$$
\left(1-u_{1}^{2}\right)-v_{2}^{2}\left(1-u_{1}^{2}\right)=\left(1-u_{1}^{2}\right)-u_{2}^{2}\left(1-v_{1}^{2}\right) \Rightarrow\left(1-v_{2}^{2}\right)\left(1-u_{1}^{2}\right)=\left(1-u_{1}^{2}\right)-u_{2}^{2}\left(1-v_{1}^{2}\right)
$$

isto é,

$$
\frac{1-v_{2}^{2}}{1-u_{2}^{2}}\left(1-u_{1}^{2}\right)=\frac{1-u_{1}^{2}}{1-u_{2}^{2}}-u_{2}^{2} \frac{1-v_{1}^{2}}{1-u_{2}^{2}}
$$

Aplicando (7.18) na equação acima obtemos

$$
\begin{aligned}
& \frac{v_{1}^{2}}{u_{1}^{2}}\left(1-u_{1}^{2}\right)=\frac{1-u_{1}^{2}}{1-u_{2}^{2}}-u_{2}^{2} \frac{1-v_{1}^{2}}{1-u_{2}^{2}} \\
\Rightarrow & v_{1}^{2}\left(1-u_{1}^{2}\right)\left(1-u_{2}^{2}\right)=u_{1}^{2}\left[\left(1-u_{1}^{2}\right)-u_{2}^{2}\left(1-v_{1}^{2}\right)\right] \\
\Rightarrow & v_{1}^{2}-v_{1}^{2} u_{1}^{2}-v_{1}^{2} u_{2}^{2}+v_{1}^{2} u_{1}^{2} u_{2}^{2}=u_{1}^{2}-u_{1}^{4}-u_{1}^{2} u_{2}^{2}+u_{1}^{2} u_{2}^{2} v_{1}^{2} \\
\Rightarrow & v_{1}^{2}\left(1-u_{1}^{2}-u_{2}^{2}\right)=u_{1}^{2}\left(1-u_{1}^{2}-u_{2}^{2}\right) \\
\Rightarrow & v_{1}^{2}=u_{1}^{2}
\end{aligned}
$$




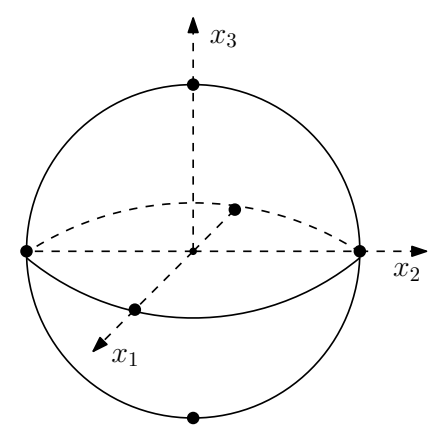

Figura 7.1: Pontos críticos de $\left.f_{2}\right|_{S^{2}}$

Como pela equação (I) $u_{1}$ e $v_{1}$ tem mesmo sinal segue que $u_{1}=v_{1}$. Além disso, de (7.19) e do fato de que $u_{2}$ e $v_{2}$ tem mesmo sinal segue que $u_{2}=v_{2}$. Portanto, pelas equações em (7.15) segue que $x_{j}=y_{j}$, como queríamos.

Tomando a parametrização onde $x_{3}=\sqrt{1-u_{1}^{2}-u_{2}^{2}}$ e $y_{3}=-\sqrt{1-v_{1}^{2}-v_{2}^{2}}$, de forma análoga ao caso anterior obtemos

$$
\nabla f_{1}\left(u_{i}, v_{i}\right)=0 \Longleftrightarrow x_{i}=-y_{i} .
$$

Dessa forma, os pontos críticos de $\widetilde{f}_{1}$ (assim como os de $f_{1}$ ) são da forma $x_{i}=y_{i}, y_{i}=-x_{i}$ e pertencem aos níveis críticos 0 e 1 , respectivamente, pois $\widetilde{f}_{1}\left[\left(x_{i}, x_{i}\right)\right]=0$ e $\widetilde{f}_{1}\left[\left(x_{i},-x_{i}\right)\right]=1$. Então, os pontos críticos de $\widetilde{f}_{1}+\widetilde{f}_{2}$ estão contidos nos planos $x_{i}=y_{i}$ e $x_{i}=-y_{i}$ e consistem dos pontos críticos de $\widetilde{f}_{2}$ restrito a eles.

Se restringirmos o domínio de $f_{2}$ a $S^{2}$, isto é, se desconsiderarmos as coordenadas $y_{i}$, podemos ver que cada uma das 6 parametrizações, análogas àquela em (7.15), que cobrem $S^{2}$ contém um ponto crítico de $\left.f_{2}\right|_{S^{2}}$. Por exemplo, tomando a parametrização como na primeira coluna em (7.15) segue que

$$
\left.\nabla f_{2}\right|_{S^{2}}\left(u_{1}, u_{2}\right)=\left(\begin{array}{ll}
2 u_{1}, & -2 u_{2}
\end{array}\right) .
$$

Logo, $(0,0,1)$ é o único ponto crítico de $\left.f_{2}\right|_{S^{2}}$ para essa parametrização. Calculando o gradiente de $\left.f_{2}\right|_{S^{2}}$ nas outras parametrizações obtemos os pontos críticos:

$$
( \pm 1,0,0),(0, \pm 1,0),(0,0, \pm 1) .
$$

Assim, os pontos críticos de $\widetilde{f}_{2}$ restrito aos planos $x_{i}=y_{i}$ e $y_{i}=-x_{i}$ são:

$$
\begin{array}{lll}
p=[(1,0,0,1,0,0)], & q=[(0,1,0,0,1,0)], & r=[(0,0,1,0,0,1)] \\
p^{\prime}=[(1,0,0,-1,0,0)], & q^{\prime}=[(0,1,0,0,-1,0)], & r^{\prime}=[(0,0,1,0,0,-1)]
\end{array}
$$

Portanto, $\widetilde{f}$ tem 6 pontos críticos $p, p^{\prime}, q, q^{\prime}, r, r^{\prime}$ e como

$$
\widetilde{f}(r)=\widetilde{f}\left(q^{\prime}\right)=0, \widetilde{f}(p)=\widetilde{f}\left(r^{\prime}\right)=1, \widetilde{f}(q)=-1, \widetilde{f}\left(p^{\prime}\right)=2
$$

segue que os níveis críticos de $\widetilde{f}$ são $-1,0,1,2$ onde existem 2 pontos críticos nos níveis 0 e 1 .

Pela Proposição 2.9 em [Tak68] existe uma função $\widetilde{f}^{\prime}$ definida em $S^{2} \times S^{2} /(x, y) \sim(-x,-y)$ que colapsa os pontos críticos de $\widetilde{f}$ que estão em um mesmo nível crítico em um único ponto crítico. Assim, $\widetilde{f}^{\prime}$ tem 4 pontos críticos. E pelas identificações em (7.14) existe uma função $g$ definida em $C^{*} / \theta$ com 4 pontos críticos.

Portanto, pelo Teorema 7.9 e a discussão feita no início dessa seção segue que a métrica Finsler $F_{\alpha}$ correspondente tem apenas 4 geodésicas de comprimento próximo de $2 \pi$ se $\alpha$ é suficientemente pequeno.

Retornando agora para a situação geral de uma variedade $M$ na qual todas geodésicas são fechadas e possuem mesmo período $2 \pi$, faremos algumas observações sobre as discussões feitas no Capítulo 5 . 
Teorema 7.19. Se tomarmos uma função $\widetilde{f}$ em $C^{*}$ tal que o fluxo de $X_{H_{1}}$ induz uma ação de $S^{1}$ em $T^{*} M$ (como foi o caso para os exemplos no Capítulo 5), então as geodésicas fechadas de $H_{\alpha}$ para $\alpha$ irracional são as geodésicas fechadas de $H_{0}$ que são invariantes sob o fluxo de $H_{1}$. Além disso, as geodésicas invariantes sob ofluxo de $H_{1}$ correspondem, exatamente, aos pontos críticos de $\widetilde{f}$.

Demonstração. Observe que como $\psi_{t}^{H_{0}}$ e $\psi_{t}^{H_{1}}$ comutam (Lema 7.7) e $\psi_{t}^{H_{1}}$ é uma $S^{1}$-órbita, então pela mesma demonstração do Lema 5.7, se $\eta \in T^{*} M$ é um ponto periódico de $\psi_{t}^{H_{\alpha}}$ tal que

$$
\psi_{T}^{H_{\alpha}} \eta=\eta
$$

segue que $\psi_{\alpha n T}^{H_{1}}$ deixa a órbita $c(t)=\psi_{-t}^{H_{0}}$ invariante para todo $n \in \mathbb{N}$. Como consequência disso, pela mesma demonstração do Lema 5.8 segue que se $\alpha T / 2 \pi$ é irracional, então $c(t)$ é invariante sob $\psi_{t}^{H_{1}}$ para todo $t \in \mathbb{R}$ e se $\alpha T / 2 \pi$ é racional então $\alpha$ é racional.

Assim, se $\alpha$ é irracional, então $\alpha T / 2 \pi$ é irracional e nesse caso $c(t)=\psi_{t}^{H_{0}} \eta$ é invariante sob $\psi_{t}^{H_{1}}$ para todo $t \in \mathbb{R}$. Então,

$$
\psi_{t}^{H_{\alpha}} \eta=\psi_{t}^{H_{0}} \circ \psi_{\alpha t}^{H_{1}} \eta=\psi_{\alpha t}^{H_{1}}(c(t))=c(h(\alpha t))
$$

para alguma função $h: \mathbb{R} \rightarrow \mathbb{R}$. Portanto, uma geodésica na co-norma Finsler $H_{\alpha}$ é uma geodésica na conorma $H_{0}$ que é invariante sob $\psi_{t}^{H_{1}}$.

Agora se $c(t)=\psi_{t}^{H_{0}} \eta$ é uma geodésica de $H_{0}$ que é invariante sob $\psi_{t}^{H_{1}}$, então precisamos mostrar que $c$ é um ponto crítico de $\widetilde{f}$. De fato, por hipótese temos

$$
\psi_{t}^{H_{1}} \eta=\psi_{t}^{H_{1}} c(0)=c(h(t))
$$

para alguma função $h: \mathbb{R} \rightarrow \mathbb{R}$. Então, se $\pi: T_{1}^{*} M \rightarrow C^{*}$, pela equação (7.21) segue que

$$
\pi\left(\psi_{t}^{H_{1}} \eta\right)=\pi(c(h(t)))=\pi(\eta), \text { para todo } t \in \mathbb{R} .
$$

Observe que $\widetilde{\eta}:=\pi(\eta)=\{c(t) ; t \in \mathbb{R}\}$. Logo, pela equação (7.6) segue

$$
\widetilde{\eta}=\pi(\eta)=\pi\left(\psi_{t}^{H_{1}} \eta\right)=\psi_{t}^{\widetilde{f}} \pi(\eta)=\psi_{t}^{\widetilde{f}} \widetilde{\eta}
$$

e como já vimos na Seção 7.1, se $\psi_{t}^{\widetilde{f}} \widetilde{\eta}$ é uma órbita trivial, então $\widetilde{\eta}$ é um ponto crítico de $\widetilde{f}$.

Antes de prosseguirmos com a discussão sobre as geodésicas na co-norma Finsler $H_{\alpha}$ vamos definir o grau de uma função suave e o índice de Poincaré-Hopf de um campo de vetores.

Sejam $X$ e $Y$ variedades de mesma dimensão. Se $h: X \rightarrow Y$ é uma aplicação suave onde $\mathrm{X}$ é uma variedade compacta e $p$ é um valor regular de $h$, então $h^{-1}(p)$ é um conjunto finito de pontos, digamos $h^{-1}(p)=\left\{x_{1}, \ldots, x_{n}\right\}$. Como $p$ é um valor regular, pelo Teorema da Função Inversa segue que em uma vizinhança de cada $x_{i}$ a aplicação $h$ é um difeomorfismo local. Difeomorfismos podem ser divididos em dois grupos: os que preservam a orientação e os que não preservam. Seja $r$ o número de pontos $x_{i}$ no qual $h$ preserva a orientação e $s$ o número no qual $h$ não preserva a orientação. Quando X é conexa, o número $r-s$ é independente da escolha de $p$ e definimos o grau de $h$ por:

$$
\operatorname{grau}(h)=r-s .
$$

Definição 7.20. Seja $p$ um zero isolado de um campo de vetores $V$ em uma variedade $M$ de dimensão $n$. Em coordenadas locais, podemos ver $V$ como uma aplicação de um aberto $U \subset \mathbb{R}^{n}$ em um aberto $U^{\prime} \subset \mathbb{R}^{n}$ onde $0 \in U, 0 \in U^{\prime}$ e tal que 0 é o único zero de $V$ em $U$. Definimos o índice de Poincaré-Hopf de $V$ em $p$ por:

$$
\operatorname{Ind}(V, p)=\operatorname{graude} \frac{V}{|V|}: S_{\varepsilon}^{n-1} \rightarrow S^{n-1},
$$

onde $S_{\mathcal{E}}^{n-1}$ é uma esfera de raio $\varepsilon>0$ contida em $U$. 
Voltando ao assunto, se $\widetilde{f}$ tem somente uma quantidade finita de pontos críticos, então qualquer zero de $X_{\widetilde{f}}$ tem índice 1 pois o fluxo de $X_{H_{1}}$ e, portanto, também o fluxo de $X_{\widetilde{f}}$ induz uma $S^{1}$-ação. Assim, pelo Teorema do índice de Hopf, a característica de Euler de $C^{*}$ é igual ao número de pontos críticos de $\widetilde{f}$ em $C^{*}$. Mas pela contagem dos números de Betti, $b_{i}(C)$, no Capítulo 5 segue que a característica de Euler de C é $2 n$ para $M=S^{2 n-1}$ ou $M=S^{2 n}, n(n+1)$ e $2 n(n+1)$ para $M=\mathbb{C} P^{n}$ e $M=\mathbb{H} P^{n}$, respectivamente. Então, não podemos obter qualquer métrica Finsler com menos geodésicas fechadas por esse método, embora as geodésicas podem ser degeneradas nessa situação mais geral.

Teorema 7.21. Se $M=S^{n}, M=\mathbb{C} P^{2}$ ou $M=\mathbb{H} P^{2}$, então, não existe uma função $\widetilde{f}$ definida em $C^{*}$ invariante sob $\theta$ e tal que o fluxo de $X_{H_{1}}$ induz uma ação de $S^{1}$ em $T_{1}^{*} M$.

Demonstração. Suponha por contradição que exista uma função como descrita no enunciado do lema. Então, $\widetilde{f}$ também está bem definida no espaço quociente $C^{*} / \theta$ e pelo mesmo argumento anterior em $C^{*} / \theta$ obtemos

$$
\frac{1}{2} \chi\left(C^{*}\right)=\chi\left(C^{*} / \theta\right)=\#\left\{\text { pontos críticos de } \tilde{f} \text { em } C^{*} / \theta\right\} .
$$

Como para $M=S^{n}, M=\mathbb{C} P^{2}$ ou $M=\mathbb{H} P^{2}$ temos

$$
\frac{1}{2} \chi\left(C^{*}\right)<\operatorname{dim} M
$$

onde M é vista como uma variedade real, então segue que toda função $\widetilde{f}$ definida em $C^{*} / \theta$ e tal que $X_{H_{1}}$ induz uma ação de $S^{1}$ em $T_{1}^{*} M$ tem uma quantidade de pontos críticos menor que a dimensão de $\mathrm{M}$.

Por outro lado, pela Proposição 2.9 e a observação abaixo dela em [Tak68] vemos que toda variedade compacta e conexa $\mathrm{N}$ admite uma função $\operatorname{com} \operatorname{dim} N+1$ pontos críticos. Logo, existe uma função $\widetilde{f}_{1}$ definida em $C^{*} / \theta$ com $2 n-1$ pontos críticos. Observe que se $\widetilde{\eta}=\pi(\eta)$ é um ponto crítico de $\widetilde{f}_{1}$ temos

$$
\psi_{t}^{\tilde{f}_{1}} \widetilde{\eta}=\widetilde{\eta}
$$

e pela equação (7.6) temos

$$
\pi(\eta)=\pi\left(\psi_{t}^{H_{1}} \eta\right)
$$

e isto implica que existe $T \in \mathbb{R}$ tal que $\psi_{T}^{H_{1}} \eta=\eta$, isto é, $\psi_{t}^{H_{1}} \eta$ é periódica. Logo, $\widetilde{f}_{1}$ é uma função tal que $X_{H_{1}}$ induz uma $S^{1}$ ação em $T_{1}^{*} M$ (nos pontos $\eta$ tais que $\pi(\eta)$ é um ponto crítico de $\widetilde{f}_{1}$ ) e tal que

$$
\#\left\{\text { pontos críticos de } \widetilde{f}_{1} \text { em } C^{*} / \theta\right\}=2 n-1>\operatorname{dim} M
$$

o que gera uma contradição.

Vimos na Proposição 7.5 que se $\widetilde{f}$ em $C^{*}$ é invariante sob $\theta$ então a métrica Finsler $F_{\alpha}$ e a co-norma Finsler $H_{\alpha}$ correspondentes são simétricas. Logo, o Lema 7.21 nos diz que, pelo menos para $M=S^{n}$, $M=\mathbb{C} P^{2}$ e $M=\mathbb{H} P^{2}$, o método usado no Lema 7.19 não pode ser usado para produzir métricas Finsler simétricas com somente uma quantidade finita de geodésicas fechadas.

\subsection{Aplicação de Poincaré das geodésicas fechadas curtas}

Por fim, iremos calcular a aplicação de Poincaré linearizada das geodésicas fechadas curtas de $H_{\alpha}$. Se $\widetilde{\eta}$ é um ponto crítico de $\widetilde{f}$ e $c(t)=\psi_{t}^{H_{\alpha}} \eta$ é a correspondente órbita periódica de $H_{\alpha}$ de comprimento $2 \pi /(1+$ $\alpha \widetilde{f}(\widetilde{\eta}))$, então podemos introduzir coordenadas como na Seção 7.1 e computar as equações diferenciais para o fluxo linearizado $d \psi_{t}^{H_{\alpha}}(\eta)$ como na Seção 6.2.

Dessa forma, seja $\left(p_{i}, q_{i}\right)$ um sistema de coordenadas simplético em $T^{*} M$ tal que $p_{1}=t$ é o parâmetro do tempo ao longo de $\psi_{t}^{H_{0}} \eta$ e tal que $q_{1}(\eta)=H_{0}(\eta)$. Aqui vamos tomar $\eta \in T_{1}^{*} M$ e então $q_{1} \equiv 1$ ao longo de $c(t)$. Se $Y(t)=\left(\xi_{i}(t), \rho_{i}(t)\right)$ é uma solução do fluxo linearizado

$$
P(Y(0))=d \psi_{t}^{H_{\alpha}}(Y(0))=Y(t),
$$


então, pelas equações em (6.12) obtemos

$$
\begin{aligned}
& \dot{\xi}_{i}(t)=\frac{\partial^{2} H_{\alpha}}{\partial q_{i} \partial q_{j}} \cdot \rho_{j}(t)+\frac{\partial^{2} H_{\alpha}}{\partial q_{i} \partial p_{j}} \cdot \xi_{j}(t) \\
& \dot{\rho}_{i}(t)=-\frac{\partial^{2} H_{\alpha}}{\partial p_{i} \partial q_{j}} \cdot \rho_{j}(t)-\frac{\partial^{2} H_{\alpha}}{\partial p_{i} \partial p_{j}} \cdot \xi_{j}(t)
\end{aligned}
$$

onde as derivadas parciais de $H_{\alpha}$ são calculadas ao longo de $c(t)$. Como $q_{1} \equiv 1$ ao longo de $c(t)$, pela equação (7.10) temos

$$
H_{\alpha}(\eta)=1+\alpha \tilde{f}(\widetilde{\eta})
$$

ao longo de $c(t)$. Assim,

$$
\begin{aligned}
& \dot{\xi}_{i}(t)=\alpha \frac{\partial^{2} \widetilde{f}}{\partial q_{i} \partial q_{j}}(\widetilde{\eta}) \cdot \rho_{j}(t)+\alpha \frac{\partial^{2} \widetilde{f}}{\partial q_{i} \partial p_{j}}(\widetilde{\eta}) \cdot \xi_{j}(t) \\
& \dot{\rho}_{i}(t)=-\alpha \frac{\partial^{2} \widetilde{f}}{\partial p_{i} \partial q_{j}}(\widetilde{\eta}) \cdot \rho_{j}(t)-\alpha \frac{\partial^{2} \widetilde{f}}{\partial p_{i} \partial p_{j}}(\widetilde{\eta}) \cdot \xi_{j}(t)
\end{aligned}
$$

Escrevendo de outra forma obtemos

$$
\left(\begin{array}{c}
\dot{\xi}_{i} \\
\dot{\rho}_{i}
\end{array}\right)=\alpha\left(\begin{array}{cc}
\widetilde{f}_{q_{i} p_{j}} & \widetilde{f}_{q_{i} q_{j}} \\
-\widetilde{f}_{p_{i} p_{j}} & -\widetilde{f}_{p_{i} q_{j}}
\end{array}\right)\left(\begin{array}{c}
\xi_{j} \\
\rho_{j}
\end{array}\right)=\alpha\left(\begin{array}{cc}
0 & I d \\
-I d & 0
\end{array}\right)\left(\begin{array}{cc}
\widetilde{f}_{p_{i} p_{j}} & \widetilde{f}_{p_{i} q_{j}} \\
\widetilde{f}_{q_{i} p_{j}} & \widetilde{f}_{q_{i} q_{j}}
\end{array}\right)\left(\begin{array}{l}
\xi_{j} \\
\rho_{j}
\end{array}\right)
$$

Logo,

$$
\left(\dot{\xi}_{i}, \dot{\rho}_{i}\right)=\alpha \cdot J \cdot A\left(\xi_{j}, \rho_{j}\right)
$$

onde

$$
J=\left(\begin{array}{cc}
0 & I d \\
-I d & 0
\end{array}\right) \quad \text { e } \quad A=H e s s \tilde{f}
$$

isto é, as equações diferenciais em (7.22) tem uma matriz coeficiente constante $\alpha \cdot J \cdot A$. Assim,

$$
\frac{d}{d t} Y(t)=\alpha \cdot J \cdot A \cdot Y(t)
$$

Observação 7.22. Uma das razões da importância da matriz exponencial é que ela pode ser usada para resolver sistemas de equações diferenciais ordinárias lineares. A solução de

$$
\frac{d}{d t} y(t)=A y(t), \quad y(0)=y_{0}
$$

onde $A$ é uma matriz constante, é dada por

$$
y(t)=\exp (A t) y_{0}
$$

Assim, pela Observação 7.22 segue que a solução de (7.23) é da forma:

$$
Y(t)=\exp (\alpha t \cdot J \cdot A) Y(0) .
$$

E como $P$ é tal que $P(Y(0))=Y(T)$, segue que

$$
P=\exp (\alpha T \cdot J \cdot A)
$$

onde $T$ é o período de $c$. 


\section{Referências Bibliográficas}

[Arn13] Vladimir Igorevich Arnol'd. Mathematical methods of classical mechanics, volume 60. Springer Science \& Business Media, 2013. 50

[Aud03] Michèle Audin. Geometry (universitext). 2003. 71

[BCS00] David Bao, S-S Chern e Zhongmin Shen. An introduction to Riemann-Finsler geometry, volume 200. Springer Science \& Business Media, 2000. 1, 15

[Bes12] Arthur L Besse. Manifolds all of whose geodesics are closed, volume 93. Springer Science \& Business Media, 2012. 23, 24, 65, 73, 98

[BT24] Raoul Bott e Loring W Tu. Differential forms in algebraic topology, volume 82. Springer Science \& Business Media, 1924. 1, 31

[BTZ82] Werner Ballmann, Gudlaugur Thorbergsson e Wolfgang Ziller. Closed geodesics on positively curved manifolds. Annals of Mathematics, páginas 213-247, 1982. 87, 89

[Dah06] Matias Dahl. An brief introduction to finsler geometry. https://math.aalto.fi/ fdahl/finsler/ finsler.pdf, 2006. Último acesso em 25/08/2016. 1

[DFBN90] B.A. Dubrovin, A.T. Fomenko, R.G. Burns e S.P. Novikov. Modern Geometry - Methods and Applications: Part 3: Introduction to Homology Theory. Graduate Texts in Mathematics. Springer, 1990. 74

[Lee48] HC Lee. Eigenvalues and canonical forms of matrices with quaternion coefficients. Em Proceedings of the Royal Irish Academy. Section A: Mathematical and Physical Sciences, volume 52, páginas 253-260. JSTOR, 1948. 73

[Mil63] J Milnor. Morse Theory (Annals of Mathematics Studies 51). Princeton, NJ: Princeton University Press, 1963. 87, 88, 89, 90

[Rob07] Colleen Robles. Geodesics in randers spaces of constant curvature. Transactions of the American Mathematical Society, páginas 1633-1651, 2007. 69

[SH13] Pedro A. S. Salomão e U. L Hryniewicz. Introdução à Geometria Finsler. Rio de Janeiro: IMPA, 2013. 1, 16, 88

[She01] Zhongmin Shen. Lectures on Finsler geometry. World Scientific, 2001. 1, 5, 16

[Tak68] Floris. Takens. The minimal number of critical points of a function on a compact manifold and the lusternik-schnirelman category. Inventiones mathematicae, 6:197-244, 1968. 98, 99, 104, 106

[Wei74] Alan Weinstein. On the volume of manifolds all of whose geodesics are closed. Journal of Differential Geometry, 9(4):513-517, 1974. 74

[Wei78] Alan Weinstein. Bifurcations and hamilton's principle. Mathematische Zeitschrift, 159(3):235248, 1978. 54, 99 
[Zi183] Wolfgang Ziller. Geometry of the katok examples. Ergodic Theory and Dynamical Systems, 3(01):135-157, 1983. iii, v, 1, 99 